\title{
Risk Preferences and their Robust Representation
}

\author{
DISSERTATION \\ zur Erlangung des akademischen Grades \\ doctor rerum naturalium \\ (Dr. Rer. Nat.) \\ im Fach Mathematik \\ eingereicht an der \\ Mathematisch-Wissenschaftlichen Fakultät II \\ Humboldt-Universität zu Berlin \\ von \\ Herrn Dipl.-Math. Samuel Drapeau \\ geboren am 23.05.1977 in Sablé sur Sarthe
}

Präsident der Humboldt-Universität zu Berlin:

Prof. Dr. Dr. h.c. Christoph Markschies

Dekan der Mathematisch-Wissenschaftlichen Fakultät II:

Prof. Dr. sc. Peter Frensch

Gutachter:

1. Prof. Dr. Hans Föllmer

2. Prof. Dr. Michael Kupper

3. Prof. Dr. Frank Riedel

eingereicht am: 15.02.2010

Tag der mündlichen Prüfung: 30.04.2010 


\begin{abstract}
The goal of this thesis is the conceptual study of risk and its quantification via robust representations.

In a first part, we consider risk within a context which extends the notion of "measurable uncertainty" introduced by Frank KnIGHT [1921]. Mathematically, the risk perception of risky elements in a convex set $\mathcal{X}$ is expressed by a preference order $\succcurlyeq$ having the properties of quasiconvexity and monotonicity. These properties are the appropriate translation of the two consensual statements that "diversification should not increase the risk" and "the better for sure, the less risky". Such a preference order will be called a risk order. We keep full latitude on the choice of the underlying setting and thus leave room for different interpretations of risk. Typical examples for $\mathcal{X}$ are the space of random variables on a given probability space, the convex set of probability distributions on the real line, or the cone of consumption streams. Risk orders can be represented by numerical representations $\rho: \mathcal{X} \rightarrow[-\infty, \infty]$ called risk measures. Any risk measure defines a level set family $\mathcal{A}=\left(\mathcal{A}^{m}\right)_{m \in \mathbb{R}}$ called risk acceptance family. Our first theorem states a one-to-one correspondence between risk orders, risk measures, and risk acceptance families. Further properties such as convexity, positive homogeneity,
\end{abstract} or cash-(sub)additivity are then characterised on these three levels.

We then study risk orders on a locally convex topological vector space $\mathcal{X}$. Our main theorem states that any lower semicontinuous risk measure $\rho$ has a unique robust representation of the form

$$
\rho(x)=\sup _{x^{*} \in \mathcal{K}^{\circ}} R\left(x^{*},\left\langle x^{*},-x\right\rangle\right) .
$$

where $R: \mathcal{K}^{\circ} \times \mathbb{R} \rightarrow[-\infty,+\infty]$ is a risk function. It is actually the leftinverse in the second argument of the minimal penalty functional $\alpha_{\min }\left(x^{*}, m\right)=$ $\sup _{x \in \mathcal{A}^{m}}\left\langle x^{*},-x\right\rangle$. Here, $\mathcal{K}^{\circ}$ is a polar convex cone in the dual space $\mathcal{X}^{*}$. The proof of uniqueness in this natural context of lower semicontinuity is technically involved, and it is new in the general theory of quasiconvex duality. We also prove a robust representation for risk measures on convex set as needed for risk orders on probability distributions or consumptions streams. We finally provide answers to the delicate question, under which circumstances monotonicity alone ensures lower semicontinuity of the risk order.

To finish this first part, we specialize our results to various typical settings. In the case of random variables, we explicitly compute the robust representation of canonical examples such as the certainty equivalent, or the economic index of riskiness. We also show that "Value at Risk" is a risk measure on the level of probability distributions and derive its robust representation. For consumption streams, we obtain a robust representation of the intertemporal utility functional of Hindy, Huang and Kreps. For stochastic kernels, we prove a general separation theorem for risk orders which distinguishes between "model risk" and "distributional risk".

In the second part of the thesis, we weaken the requirement of completeness of the preferences, that is, the necessity of deciding whether one element is preferable or not to the other. We introduce the concept of a preference order which might require additional information in order to be expressed. In a first section we 
provide a mathematical framework for this idea in terms of preorders which are locally compatible with the given information as described by a $\sigma$-algebra $\mathcal{G}$. Such preorders will be called conditional preference orders. Using Zorn's lemma, we can lift this local information compatibility to a global level. This allows us to construct conditional numerical representations of conditional preferences.

Restricting our analysis to the level of $\mathscr{G}$-measurable stochastic kernels, we obtain a conditional version of the von Neumann and Morgenstern representation the form

$$
\tilde{u}(\mu)=\int u(\cdot, x) \mu(\cdot, d x)
$$

The main difficulty here is the proof of the $\mathcal{G}$-almost sure continuity of the conditional utility function $u$. We then extend our study to the case of general stochastic kernels. We formulate a conditional version of the variational preferences introduced by MACCHERONI ET AL. [2006A], and we prove a representation of the form

$$
\tilde{U}(\tilde{X})=-\underset{Q}{\operatorname{ess} \sup _{Q}}\left\{E_{Q}\left[-\int u(\cdot, x) \tilde{X}(\cdot, d x) \mid \mathscr{G}\right]-\alpha_{\min }(Q)\right\} .
$$

This representation combines the conditional affine part à la von Neuman and Morgenstern on the level of distributions with a conditional cash additive risk measure on the level of random variables and thus clarifies the interplay between model risk and distributional risk. Finally, we formulate additional axioms which characterize the two cases of "pure" model risk or "pure" distributional risk.

Key Words: Risk Preference, Risk Order, Risk Measure, Risk Acceptance Family, Robust Representation, Conditional Preference, Value at Risk, Certainty Equivalent, von Neuman and Morgenstern Representation, Automatic Continuity, Economic Index of Riskiness. 



\section{Zusammenfassung}

Ziel dieser Dissertation ist es, den Begriff des Risikos unter den Aspekten seiner Quantifizierung durch robuste Darstellungen zu untersuchen.

In einem ersten Teil wird Risiko in einem weitgespannten Rahmen betrachtet, der den von FRANK KNIGHT [1921] eingeführten Begriff der "messbaren Ungewissheit" deutlich erweitert. Mathematisch wird Risikowahrnehmung von riskanten Elementen einer konvexen Menge $\mathcal{X}$ durch eine Präferenzordnung $\succcurlyeq$ präzisiert. Um die mit Risiko verbundenen Merkmale "Diversifizierung sollte das Risiko nicht erhöhen" und "Desto besser, umso weniger riskant" auszudrücken, hat diese Präferenzordnung die Eigenschaften der Quasikonvexität und der Monotonie. Eine solche Präferenzordnung wird Risikoordnung genannt.

Diese Herangehensweise lässt bei der Wahl der konvexen Menge viel Spielraum, und erlaubt damit eine Vielfalt von Interpretationen von Risiko. Typische Beispiele für solche $\mathcal{X}$ sind der Vektorraum der Zufallsvariablen auf einem Wahrscheinlichkeitsraum, die konvexe Menge der Wahrscheinlichkeitsverteilungen auf der reellen Achse oder auch der Kegel der Konsumströme. Risikoordnungen haben eine numerische Darstellung durch eine Funktion $\rho: \mathcal{X} \rightarrow[-\infty,+\infty]$, auch Risikomaß genannt. Ein solches Risikomaß definiert eine Niveaumengen-Familie $\mathcal{A}=\left(\mathcal{A}^{m}\right)_{m \in \mathbb{R}}$, die wir Risikoakzeptanzfamilie nennen. Das erste Theorem stellt eine eins-zu-eins Beziehung zwischen Risikoordnung, Risikomaßen und Risikoakzeptanzfamilen her. Weitere Eigenschaften wie Konvexität, positive Homogenität und Cash (sub)Additivität werden dann auf diesen drei Ebenen charakterisiert.

Wir untersuchen dann Risikoordnungen auf lokal konvexen topologischen Vektorräumen. Unser Hauptresultat zeigt, dass jedes unterhalbstetige Risikomaß $\rho$ eine eindeutige robuste Darstellung von folgender Form hat:

$$
\rho(x)=\sup _{x^{*} \in \mathcal{K}^{\circ}} R\left(x^{*},\left\langle x^{*},-x\right\rangle\right)
$$

wobei $R: \mathcal{K}^{\circ} \times \mathbb{R} \rightarrow[-\infty,+\infty]$ eine Risikofunktion ist. In der Tat ist $R$ die im zweiten Argument rechte Inverse der minimalen Penalitätsfunktion $\alpha_{\min }\left(x^{*}, m\right)=$ $\sup _{x \in \mathcal{A}^{m}}\left\langle x^{*},-x\right\rangle$. Hier ist $\mathcal{K}^{\circ}$ ein polarer Kegel im Dualraum $\mathcal{X}^{*}$. Der Beweis der Eindeutigkeit im unterhalbstetigen Fall ist die eigentliche technische Herausforderung; er ist auch in der allgemeinen Theorie der quasiconvexen Dualität neu. Wir zeigen auch robuste Darstellungen für Risikomaße auf konvexen Mengen, wie sie bei Risikoordnungen auf Wahrscheinlichkeitsverteilungen oder auf Konsumströmen auftreten. Anschließend geben wir Antworten auf die komplizierte Frage, unter welchen Annahmen die Monotonie die Unterhalbstetigkeit impliziert.

Im dritten Abschnitt wenden wir unsere Ergebnisse auf verschiedene typische Situationen an. Bei den Zufallsvariablen behandeln wir die Fatou Eigenschaft und berechnen explizit die robuste Darstellung von einigen kanonischen Beispielen wie Sicherheitsäquivalenten, oder ökonomischen Risikoindices. Wir zeigen, dass "Value at Risk" ein Risikomaß auf der Menge der Wahrschenlichkeitsverteilungen ist und berechnen seine robuste Darstellung. Bei den Konsumströmen berechnen wir die robuste Darstellung des von Hindy et al. eingeführten intertemporalen Nutzenfunktionals. Für Risikoordnungen auf stochastischen Kernen zeigen wir einen Trennungssatz, der zwischen Modellrisko und Verteilungsrisiko unterscheidet. 
Im zweiten Teil dieser Dissertation, schwächen wir die Annahme der Vollständigkeit der Praferenzordnungen ab, also die Notwendigkeit, zwischen zwei Elementen entscheiden zu müssen. Hierzu führen wir einen Axiomatic ein, die Unvollständikeit zulässt und in der die Präferenzordnugen lokal kompatibel mit der von einer $\sigma$ Algebra $\mathscr{G}$ modellierten Information, sind. Solche Präferenzen nennen wir bedingte Präferenzordnungen. In diesem Kontext, zeigen wir mit Hilfe von Zorn's Lemma, dass diese lokale Spezifikation auch eine globale Formulierung zulässt. Dies erlaubt die Konstruktion einer bedingten numerischen Darstellung.

Durch Einschränkung unserer Untersuchung auf die Ebene der $\mathscr{G}$-meßbaren stochastichen Kerne, erhalten wir eine bedingte Variante der von Neumann und Morgenstern Darstellung in der Form:

$$
\tilde{u}(\mu)=\int u(\cdot, x) \mu(\cdot, d x) .
$$

Hier liegt die Schwierigkeit vor allem im Beweis der $\mathcal{G}$-fast sicher Stetigkeit der Nutzenfunktion $u$. Wir erweitern dann der Rahmen auf allgemeine stochastiche Kerne und formulieren eine bedingten Variante der von MACCHERONI ET AL. [2006A] eingeführten "Variational Preferences". Dies führt zu einer Darstellung folgender Art:

$$
\tilde{U}(\tilde{X})=-\underset{Q}{\operatorname{ess} \sup _{Q}}\left\{E_{Q}\left[-\int u(\cdot, x) \tilde{X}(\cdot, d x) \mid \mathscr{G}\right]-\alpha_{\min }(Q)\right\}
$$

Diese Darstellung kombiniert die bedingte Version der von Neuman and Morgenstern Darstellung auf die Ebene der Wahrscheinlichkeitsverteilungen mit einem konvexen translationsinvarianten Risikomß auf die Ebene der Zufallsvariablen, und klärt so das Zusammenspiel von Modelrisiko und Verteilungsrisiko. Abschließend formulieren wir zusätzliche Bedingungen, die die beiden Fälle eines reinen Modellrisikos oder eines reinen Verteilungsrisikos charakterisieren.

Schlagwörter: Riskopräferenz, Riskoordnung, Riskomaß, Risikoakzeptanzfamilen, Robuste Darstellung, Bedingte Präferenz, Value at Risk, Sicherheitsäquivalent, ökonomischer Risikoindex, von Neuman and Morgenstern Darstellung, Automatische Stetigkeit 


\section{Contents}

$\begin{array}{lr}\text { Introduction } & 1\end{array}$

I. Risk Preferences and their Robust Representation 17

1. Risk Orders 19

1.1. Risk Orders, Risk Measures and Risk Acceptance Families . . . . . . . . 19

1.2. Convexity, Positive Homogeneity, Scaling Invariance . . . . . . . . . . . 28

1.3. Affine Risk Orders . . . . . . . . . . . . . . . . . . . . . 30

1.4. Monetary Risk Orders . . . . . . . . . . . . . . . . . 31

2. Robust Representation of Risk Orders 37

2.1. A General Robust Representation Result . . . . . . . . . . . . . . . . . . . 37

2.2. Proof of the General Robust Representation . . . . . . . . . . . . . . . . . . 43

2.3. Special Cases . . . . . . . . . . . . . . . . . . . . 51

2.4. Robust Representation of Risk Orders on Convex Sets . . . . . . . . . . 52

2.5. Automatic Continuity Results . . . . . . . . . . . . . . . . . 55

2.5.1. $\pi$-Bounded Preferences . . . . . . . . . . . . . 55

2.5.2. Affine Risk Measures on $\mathcal{M}_{1, c} \ldots \ldots \ldots$. . . . . . . 56

3. Illustrative Settings $\quad \mathbf{6 5}$

3.1. Random Variables . . . . . . . . . . . . . . . . . . 65

3.2. Probability Distributions . . . . . . . . . . . . . . . . 73

3.3. Consumption Streams . . . . . . . . . . . . . . . . . . 76

3.4. Stochastic Kernels . . . . . . . . . . . . . . . . . . . 79

II. Conditional Preferences $\quad 89$

$\begin{array}{ll}\text { 4. Conditional Preferences } & 91\end{array}$

4.1. Axiomatic and First Results . . . . . . . . . . . . . . . . . . . . . 91

4.2. Conditional Variational Preferences . . . . . . . . . . . . . . . . 97

5. Conditional Robust Representations 101

5.1. A Conditional version of the von Neumann and Morgenstern Representation 101

5.2. Robust Representation of the Conditional Variational Preferences . . . . 108

5.3. Distributional Risk, Model Risk . . . . . . . . . . . . . . . . 117 



\section{Introduction}

Risk is now a colloquial and widely used term. Nevertheless, its emergence in history is relatively recent. While the term "risicum" already appears in the Middle Ages in highly specific contexts, LUHMANN [1996] traces its wider use and the diversification of its meaning to the early Renaissance and writes in [Luhmann, 2002, Page 16]

"The late apparition in history of circumstances indicated by means of the new term 'risk' is probably due to the fact that it accommodates a plurality of distinctions within one concept, thus constituting the unity of this plurality."

This plurality is probably the reason why, when it comes to define the concept of risk, ways are parting and no real consensus has emerged; as Luhmann [1996, Page 4] observes, "the concept of risk remains unclear even today." While the Oxford English Dictionary links risk to "hazard, danger; exposure to mischance or peril", many other terms gravitate around this idea such as fortune, safety, prudence, losses, vulnerability, decision, opportunity, uncertainty, or contingency. Scientific areas ranging from economics, finance, sociology and psychology to medicine, physics, or engineering have laid claim to this concept. They do so with their own instruments, language, and objectives focusing on different "kinds" of risk such as operational risk, financial risk, social risk, political risk, managerial risk, or nuclear risk to name but a few.

On a mathematical level, methods of assessing risk in a quantitative manner were developed in the early stages of probability theory. A famous example is the work of Cramer (1728) and Bernoullli (1738) on the St. Petersburg paradox. Here they introduce the idea of expected utility, assessing a risky monetary venture by a sum of utilities of its possible outcomes weighted by their respective probabilities, see [Bernoulli, 1954, Fishburn, 1988]. In a financial context, other key concepts related to the quantification of risk are the variance, as in the celebrated mean variance criterion of MARKOWITZ [1952] and in SHARPE [1964]'s ratio, or the quantiles of a loss distribution as in the case of "Value at Risk".

In this thesis, we focus on the mathematical analysis of risk by means of preference orders. Such an approach starts with a set of consistent rules or axioms for preferences on a given set of risky elements. These axioms express a normative view of rationality, in the sense that a reasonable person, if confronted to this set of rules, is expected to agree with these guidelines. This method originates in the mid twentieth century with the celebrated work of von Neumann And Morgenstern [1947]. They analysed preferences on a set of "lotteries", which specify a probability distribution $\mu$ of possible real valued outcomes. If these preferences satisfy the Archimedian axiom, the independence axiom, the monotonicity axiom and some continuity, they admit a numerical 


\section{INTRODUCTION}

representation of the form

$$
U(\mu)=\int u d \mu,
$$

with some continuous nondecreasing "utility function" $u$. Under the additional assumption of risk aversion, the function $u$ is also concave and thus a utility function in the classical sense. From a descriptive point of view, however, there are good reasons not to insist on the concavity of $u$, as demonstrated by numerous empirical studies and behavioral experiments, see for instance [Kahneman and Tversky, 1979, Kahneman, 2000].

The axiomatic approach of von NEUMAnN AND Morgenstern paved the way to modern economic theory and financial mathematics. A first significant extension of this work is the representation of SAVAGE [1972], where the previous axioms are formulated on the level of measurable functions $X$ on a set $\Omega$ of possible scenarios and yield a numerical representation

$$
U(X)=E_{Q}[u(X)],
$$

with some utility function $u$ and some "subjective" probability measure $Q$ which is implicit in the preferences. Many generalizations of this paradigm of "expected utility" were given in the sequel, in particular an extension by ANSCOMBE AND AUMANn [1963] to stochastic kernels of the form

$$
U(\tilde{X})=E_{Q}\left[\int u(x) \tilde{X}(\cdot, d x)\right]
$$

where $\tilde{X}(\cdot, d x)$ is a stochastic kernel from $\Omega$ to the real line. These stochastic kernels can be seen as scenario dependent lotteries and thus provide a unifying framework both for measurable functions and lotteries. A survey of these extensions can be found in [Fishburn, 1988].

An important step beyond the classical paradigm of expected utility is the axiomatic approach of GILBOA AND SCHMEIDLER [1989], formulated in the setting of stochastic kernels. On the level of random variables, the resulting numerical representation takes the form

$$
U(X)=\inf _{Q \in \mathcal{Q}} E_{Q}[u(X)],
$$

for a whole class $\mathcal{Q}$ of probability models. Such a representation, which takes into account more than one probability model, can be seen as a robust version of the classical expected utility (I).

At the end of the twentieth century, again in the spirit of a normative approach, Artzner, Delbaen, Eber, and Heath introduced in their seminal paper [Artzner et al., 1999] the concept of a coherent cash additive risk measure. This was motivated by the need of regulatory agencies for a method of specifying capital requirements for financial institutions, and by the insight that the standard method of "Value at Risk" has serious deficiencies, both on a conceptual and practical level. A typical coherent 
cash additive risk measure takes the form

$$
\rho(X)=\sup _{Q} E_{Q}[-X]
$$

for some class $\mathcal{Q}$ of probability measures. Note that, from this point of view, the GiLBOA AND SCHMEIDLER representation can be written as

$$
U(X)=-\rho(u(X))
$$

for some coherent risk measure $\rho$.

A coherent risk measure can be described as a convex cash additive risk measure which is also positively homogeneous. The general notion of convex cash additive risk measure was introduced independently by Föllmer AND SCHIED [2002], FRITTELli AND Rosazza Gianin [2002] and Heath [Paris 2000]. Designed for random variables, the axioms for a convex cash additive risk measure are

- monotonicity: a financial position which is better in any scenario than another has a lower risk,

- cash additivity: adding some money to a financial position lowers its risk by exactly this amount,

- convexity: the risk of a convex combination is lower than the convex combination of their respective risks.

Under some additional regularity assumptions, these axioms yield a representation

$$
\rho(X)=\sup _{Q}\left\{E_{Q}[-X]-\alpha(Q)\right\}
$$

where expected losses $E_{Q}[-X]$ are computed under different probabilistic models $Q$. These models may be more or less plausible, and this is specified by some penalty term $\alpha(Q)$. Taking a "worst case" approach, the monetary risk $\rho(X)$ of the position $X$ is then defined by the supremum in (III). The representation (II), now with a convex rather than a coherent cash additive risk measure $\rho$, characterizes the so called variational preferences introduced by MACCHERONI, MARINACCI, AND Rustichini [2006A]. A survey article by Föllmer, SCHIED, AND WEBER [2009] deals with this representation and its application to various robust optimizations problems.

Convex cash additive risk measures were further investigated in the context of numéraire uncertainty. There, the assumption of cash additivity is debatable as argued by EL Karoui AND Ravanelli [2009], who proposed the weaker assumption of cash subadditivity. Later, Cerreia-Vioglio, Maccheroni, Marinacci, and MontrucCHIO [2010], pointing out that quasiconvexity rather than convexity is the appropriate mathematical translation of the statement "diversification should not increase the risk", introduced the notion of a quasiconvex cash subadditive risk measure. For these risk 


\section{INTRODUCTION}

measures they derived the representation

$$
\rho(X)=\sup _{Q} R\left(Q, E_{Q}[-X]\right),
$$

where the function $R(\cdot, \cdot)$ is increasing in the second argument and jointly quasiconcave. In the cash-invariant case, the function $R$ takes the form $R(Q, s)=s-\alpha(Q)$, and then (IV) reduces to (III).

Let us now come back to the first quarter of the twentieth century and discuss, in view of those different stages of the normative approach, the famous distinction between risk and uncertainty proposed by KNIGHT [1921, Part III, Chapter VIII, Paragraph 1]

"To preserve the distinction [...] between the measurable uncertainty and an unmeasurable one we may use the term 'risk' to designate the former and the term 'uncertainty' for the latter. [...] The practical difference between the two categories, risk and uncertainty, is that in the former the distribution of the outcome in a group of instances is known (either through calculation a priori or from statistics of past experience), while in the case of uncertainty this is not true"

Thus, KNIGHT restricts the notion of risk to situations where an "objective" probability can be assigned to uncertain outcomes. On the other hand, "unmeasurable uncertainty" is what KeYNES [1937, Pages 213 - 214], in a response to objections to his book "The General Theory of Unemployment, Interest and Money" 1936, later describes as follows:

"By 'uncertain' knowledge, let me explain, I do not mean merely to distinguish what is known for certain from what is only probable. The game of roulette is not subject, in this sense, to uncertainty; nor is the prospect of a Victory bond being drawn. [...] The sense in which I am using the term is that in which the prospect of a European war is uncertain, or the price of copper and the rate of interest twenty years hence, or the obsolescence of a new invention, or the position of private wealthowners in the social system in 1970. About these matters there is no scientific basis on which to form any calculable probability whatever. We simply do not know."

The Knightian distinction between uncertainty (unmeasurable uncertainty) and risk (measurable uncertainty involving a specific probabilistic model) has had a strong impact on modern economic thought. It also appears in the "The Turner Review: A regulatory response to the global banking crisis" (2009) by the Britisch Financial Service Authority, where "Knightian uncertainty" is contrasted with "mathematically modelable risk" [Lord Turner, 2009, Page 45]. But the recent development in the mathematical theory of preferences, which we have sketched above, suggests to define the notions of "risk" and of "measurable uncertainty" in a much broader sense, far beyond the classical setting of a single probabilistic model.

In the following, we understand uncertainty merely as the fact that future situations might have more than one possible scenario. As in KNIGHT [1921], we retain the notion 
of unmeasurable uncertainty for situations where, in the words of KEYNES above, "We simply do not know." On the other hand, we extend the notion of "measurable uncertainty" to any situation where some quantification takes place. Such a quantification will typically involve a whole set of probabilistic models, as illustrated by the theory of convex cash additive risk measures and by GILBOA AND SCHMEIDLER's robustification of expected utility.

In this context of measurable uncertainty, our discussion of risk will involve some further quantitative features. To begin with, risk is definitively a subjective notion as stated by Luhmann [1996, Page 6]:

"[...] causal terms and terms like risk or danger are not indications of ontological facts about which one can have only true or false opinions. [...] Risk evaluation is not simply a problem of avoiding an error. The question rather is: who uses which frame to guide his observations; and then, who observes others handle causal distinctions and how they discriminate external and internal attribution depending upon whether they themselves or other make the decisions."

Thus, risk is a matter of perception. In contrast to LUHMANN, however, we do not want to subordinate risk to decision making. Indeed, risk concerns could arise prior to one's decision, but this decision might in fact not correspond to the risk assessment. After severe losses, some traders execute extreme gambles in perfect knowledge of the riskiness of such moves, bypassing a more prudent behavior their risk assessment should have called for.

Let us now focus on two key properties one usually relates to risk perception, independently of the specific context. They are expressed by the statements "diversification should not increase the risk" and "the better for sure, the less risky". There is a broad consensus that they capture crucial features of risk perception. Furthermore, they leave full latitude in which setting risk could be considered and how "diversification" or "better for sure" might be specified, in accordance with LuHMANN's quotation in the first paragraph.

This emphasis on the perception of risk motivates the mathematical approach we will develop in the first part of this thesis. This part is based on joint work with KUPPER in [Drapeau and Kupper, 2010]. It is inspired by CERREIA-VIOGLIO, MACCheroni, MARINACCI, AND MonTRUCCHIO [2008A,B, 2010], in particular by their systematic use of quasiconvexity. We also use techniques and concepts from the theory of monetary convex risk measures mentioned previously, and from the general theory of preferences, for which we refer to [Fishburn, 1988, Föllmer and Schied, 2004] and the numerous references therein.

In the first chapter, the perception of risk is expressed in terms of a preference order $\succcurlyeq$ on a convex $\operatorname{set}^{1} \mathcal{X}$ of elements of prospective nature where the relation $x \succcurlyeq y$ means that "the element $x$ is perceived to be riskier than the element $y$ ". In order to reflect

${ }^{1}$ The weaker framework of mixture space could have been considered as well. However, up to two reasonable additional conditions (non triviality, and a weak form of associativity), any mixture spaces can be embedded as a convex subset of a vector space, see [Mongin, 2000]. 


\section{INTRODUCTION}

the aforementioned key properties of risk perception that "diversification should not increases the risk" and that "the better for sure, the less risky", this preference order should be quasiconvex and monotone. The monotonicity is formulated with respect to a given preorder on $\mathcal{X}$, which can actually be trivial depending on the specific context. Such quasiconvex and monotone preference orders will be called risk orders. The meaning of diversification and monotonicity strongly depends on the given setting and thus leaves room for different interpretations of risk. Typical settings where different types of diversification occur are the set of random variables, where convex combinations are defined pointwise, and the set of lotteries where a convex combination corresponds to an additional randomization.

Any numerical representation of these risk orders inherits the properties of quasiconvexity and monotonicity. In general, a functional

$$
\rho: \mathcal{X} \longrightarrow[-\infty,+\infty],
$$

having these two properties will be called a risk measure. To any such risk measure, we associate the risk acceptance family denoted by $\mathcal{A}=\left(\mathcal{A}^{m}\right)_{m \in \mathbb{R}}$ where

$$
\mathcal{A}^{m}=\left\{x \in \mathcal{A}^{m} \mid \rho(x) \leq m\right\},
$$

is the set of those elements whose risk is lower than $m$. Independently from any risk measure we introduce the general concept of a risk acceptance family by the axioms of convexity, monotonicity and right continuity on the level of sets. The main result of this first chapter is Theorem 1.10 which clarifies the correspondence between these three concepts of risk order, risk measures, and risk acceptance families. More precisely, any risk measure $\rho$ induces a risk order $\succcurlyeq$ via

$$
x \succcurlyeq y \quad \Longleftrightarrow \quad \rho(x) \geq \rho(y) .
$$

Moreover, any risk acceptance family defines a risk measure via

$$
\rho(x)=\inf \left\{m \in \mathbb{R} \mid x \in \mathcal{A}^{m}\right\},
$$

and, as a consequence, induces a risk order via

$$
x \succcurlyeq y \quad \Longleftrightarrow \quad \text { for any } m, x \in \mathcal{A}^{m} \text { implies } y \in \mathcal{A}^{m} .
$$

We also explain in which sense the correspondences are in fact one-to-one. Risk measures derived from a family of acceptance sets were already used in [Cherny and Madan, 2009, Brown et al., 2009]. However, our definition of a risk acceptance family includes additional properties which allow us to establish the one-to-one correspondence with risk measures. The risk acceptance family is not only a powerful tool to show that a functional is a risk measure. In a given economic setting, it is often natural to start with the notion of risk acceptance, and this yields new examples of risk measures. 
It is moreover a key instrument in the computation of robust representations of risk measures. Among the typical risk measures presented as examples, we introduce a new general version of the economic index of riskiness introduced by Aumann AND SERRANO [2008].

In section 1.2 of Chapter 1, we study additional properties such as convexity, positive homogeneity, or scaling invariance which a risk measure might have, and we show how they can be expressed in terms of the risk acceptance family. Unlike quasiconvexity or monotonicity, these properties are no longer global in the sense that they do not characterize the entire class of risk measures associated to a given risk order; only a subset of the numerical representations representing the risk order might share these properties. This is in particular the case for the affine risk measures introduced in section 1.3, even though the existence of such a risk measure can be guaranteed on the level of the risk order by the Archimedian axiom and the independence axiom. In the final section 1.4 of this first Chapter, we introduce the concept of monetary risk measures, which involves the notions of cash additivity and cash subadditivity, in the general context of vector spaces. Theorem 1.22 states necessary and sufficient conditions for a risk order to be represented at least by one cash additive risk measure. Here again this property is not a global one. We also show how the properties of cash additivity and cash subadditivity are reflected on the level of risk acceptance families. It is well known that cash additivity together with quasiconvexity implies convexity, and we provide a simple proof of this fact using the risk acceptance family. We finish this section by introducing two examples of monetary risk measures which have a natural economic motivation on the level of the risk acceptance family: The case of multiple numéraires and the case of liquidity costs.

In our second chapter, the goal is to derive a dual representation of risk orders. To this end, we choose the framework where $\mathcal{X}$ is a locally convex topological vector space. It is a nontrivial result by Bosi AND MEHTA [2002] that lower semicontinuous preference orders admit a lower semicontinuous numerical representation. We use this result to state that any lower semicontinuous risk order can be represented by a lower semicontinuous risk measure. Furthermore, the class of lower semicontinuous risk measures is stable under lower semicontinuous increasing transformation, and in this sense lower semicontinuity can be considered as a global topological property. Our first result is Theorem 2.7. It states that any lower semicontinuous risk measure admits a unique robust representation

$$
\rho(x)=\sup _{x^{*} \in \mathcal{K}^{\circ}} R\left(x^{*},\left\langle x^{*},-x\right\rangle\right)
$$

where the function $R: \mathcal{K}^{\circ} \times \mathbb{R} \rightarrow[-\infty,+\infty]$ is, up to two other technical properties, jointly concave, non decreasing and left-continuous in the second argument, and such that the right-continuous version $R^{+}$is upper semicontinuous in the first argument. Such a function $R$ will be called a maximal risk function. The set $\mathcal{K}^{\circ}$ is the polar cone in the dual space $\mathcal{X}^{*}$ determined by the given preorder on $\mathcal{X}$ which is used to define the monotonicity of the risk order. If the preorder is trivial then the polar cone is the entire dual space $\mathcal{X}^{*}$. In the case where $\mathcal{X}$ is the set of bounded random variables, 


\section{INTRODUCTION}

and monotonicity refers to the preorder "greater than $P$-almost surely", the polar cone $\mathcal{K}^{\circ}$ is the set of positive integrable random variables. From a decision theoretical point of view, the uniqueness of $R$ is crucial since it allows one to do comparative statics in terms of the risk function $R$. The proof of the robust representation involves the minimal penalty functional

$$
\alpha_{\min }\left(x^{*}, m\right)=\sup _{x \in \mathcal{A}^{m}}\left\langle x^{*},-x\right\rangle,
$$

and this emphasizes the crucial role of the risk acceptance family. The minimal penalty functional is nondecreasing in the second argument, and its left inverse yields the maximal risk function. The second main result is Theorem 2.8. It states that the supremum in $(\mathrm{V})$ can be taken only over normalized elements of $\mathcal{K}^{\circ} \backslash\{0\}$ if the preorder satisfies some regularity conditions. In the case where $\mathcal{X}$ is the set of bounded random variables, we can reduce the supremum to the set of integrable positive random variables with expectation one, and so we obtain a representation in terms of probability measures.

In the context of general quasiconvex functionals with certain topological properties (even quasiconvexity which is slightly more general than lower semicontinuity), duality results of the form (V) were already given by Penot And Volle [1990A]. They use various techniques, and in one of them support functions are involved which can be identified with our minimal penalty functional. They do not address, however, the questions of monotonicity and uniqueness. Recently, Cerreia-Vioglio, MaCCHERONI, MARINACCI, AND MontruCChIO [2008B] proved the uniqueness of this representation for a regular preorder in the setting of $M$-spaces ${ }^{2}$. In this special setting, their result is more general than Theorem 2.8 since it holds for evenly quasiconvex functionals, but they do not characterise the lower semicontinuous case. In contrast, our Theorems 2.7 and 2.8 apply to the general setting of locally convex vector spaces. In the case of Theorem 2.7, it even holds for a nonregular preorder, such as the first stochastic order for lotteries. It establishes moreover the unique characterisation of the lower semicontinuous case. This is a delicate point since one has to use the right-continuous version of $R$, as illustrated by two examples in Remark 2.10 where only $R^{+}$and not $R$ is upper semicontinuous. As to the maximal risk function $R$, the term "maximal" is justified by Proposition 2.9 which states that $R$ is pointwise maximal in an appropriate class of risk functions. The proofs of Theorems 2.7 and 2.8 are given in section 2.2.

In section 2.3, we derive specific representations in the cases where the risk measure $\rho$ is additionally convex, positive homogeneous, scaling invariant, affine, or cash(sub)additive. In section 2.4, we prove a representation theorem in the case where $\mathcal{X}$ is not a vector space but either a closed or an open convex set. This Theorem 2.19 covers the important cases when $\mathcal{X}$ is a set of lotteries with varying compact support, or a class of consumption streams. The last section 2.5 of this second chapter is inspired by BoRweIn [1987]. We prove two theorems where, under some conditions on the preorder and the topology, the lower semicontinuity of the risk order is in fact a

\footnotetext{
${ }^{2}$ A typical example of an $M$-space is the set of bounded random variables. On the other hand, the space of $p$-integrable random variables is not an $M$-space if $p<+\infty$.
} 
consequence of its monotonicity. If the risk order is defined on a Fréchet space, only a single directional lower semicontinuity is sufficient. In the case of affine risk orders on lotteries with varying compact support, the situation is far more complicated. Here $\mathcal{X}$ is neither a Fréchet space nor compact in the space of signed measures. Nevertheless we are able to show that any affine risk order which is monotone with respect to the first stochastic order, and satisfies some additional assumptions on the one dimensional subspace of Dirac measures, is automatically weakly lower semicontinuous. It follows that the risk order admits a vON NEUMANN AND MORGENSTERN representation even though we have not assumed global weak continuity of the risk order, in contrast to the standard literature, see for instance [Föllmer and Schied, 2004, Chapter 2].

The third chapter is devoted to the applications of our representation results in the various special settings introduced in the first chapter. It illustrates in particular how the constructive approach used in the proof allows one to compute explicitly the maximal risk function $R$. In a first section, we study the case where $\mathcal{X}$ is a special convex subset of random variables, and discuss in particular the topological problems related to the Fatou property. Due to the robust representation, risk perception in this context can be interpreted as model risk. We then compute the maximal risk function appearing in the robust representation of the classical certainty equivalent for various utility functions, of various economic indices of riskiness, and finally for the case of liquidity costs. In section 3.2, we consider the setting of lotteries with varying compact support. Here, we also derive a unique robust representation by way of which we can interpret risk perception as distributional risk. In this context, we also discuss the "Value at Risk", which is not quasiconvex on the level of random variables and hence not a risk measure in our sense. But we show, that, considered on the level of probability distributions, it is in fact quasiconvex but not convex, and monotone with respect to the first stochastic order. It is thus a risk measure on the level of probability distributions. In the following section 3.3, we consider risk orders on consumption streams, and derive also a robust representation result which in this context allows an interpretation of risk perception as discounting risk. As an example we show that the intertemporal utility functionals proposed by HiNDY, HUANG, AND KREPS [1992] are risk measures, and we compute their robust representations in some special cases. In our final section 3.4 of this third chapter, we study the interplay between model risk and distributional risk in the case where $\mathcal{X}$ is a class of stochastic kernels. Theorem 3.18 states that both dimensions of risk can be separated under an additional assumption of monotonicity. In the restricted setting of affine risk orders on lotteries, we recover under weaker assumptions the results about uncertainty preferences in [Cerreia-Vioglio et al., 2008a] and the so called variational preferences in [Maccheroni et al., 2006a]. In particular we obtain with the Fatou property a robust representation in terms of probability measures instead of finitely additive measures.

In the second part of this work, we carry the preceding analysis one step further. In the normative framework of the first part we have used two axioms in the definition of a total preorder, completeness and transitivity. But there are good reasons to question the axiom of completeness, as already pointed out by AumanN [1962]: 


\section{INTRODUCTION}

Of all the axiom of utility theory, the completeness axiom is perhaps the most questionable. Like others of the axioms, it is inaccurate as a description of real life, but unlike them we find it hard to accept even from a normative viewpoint. [...] For example, certain decisions that an individual is asked to make might involve highly hypothetical situations, which he will never face in real life. He might feel that he cannot reach an "honest" decision in such cases. Other decision problems might be extremely complex, too complex for intuitive "insight", and our individual might prefer to make no decision at all in these problems. Is it "rational" to force decision in such cases?

As suggested by AumanN's observation, we develop an axiomatic setting where completeness is no longer required. Our guiding idea is that although an individual may not be able to rank two positions directly, he may be able to do so if he were given enough informations.

From a mathematical point of view, it is natural to formulate this issue in terms of conditional preferences with respect to a given sigma-algebra $\mathscr{G}$ modelling the available additional information. The consideration of conditional preferences and their related numerical representations is relatively recent in comparison to the long history of the standard theory. A first milestone in this direction is the contribution of SkIADAS $[1997 \mathrm{~A}, \mathrm{~B}]$, where he gives an axiomatic analysis of conditional preferences on random variables which admit a conditional Savage representation of the form

$$
U_{\mathscr{G}}(X)=E_{Q}[u(X) \mid \mathscr{G}] .
$$

The key point of his Ansatz is to consider a total preorder $\succcurlyeq^{G}$ for each event $G \in \mathscr{G}$, and a consistent way of aggregating the whole family $\left(\succcurlyeq^{G}\right)_{G \in \mathscr{G}}$ in order to obtain the conditional representation. Later, MACCHERONI ET AL. [2006B] extended their results for variational preferences to a dynamical setting with a simple finite tree structure using SKIADAS's approach. Note, however, that each preference relation $\succcurlyeq^{G}$ is assumed to be complete. In particular, this is the case for $G=\Omega$ where no additional information is available. Thus SKIADAS' Ansatz does not really address the issue raised by Aumann's observation. Note also that the set of preference relations to take into account would be huge even for finite sets since it grows with the information set and not the state set ${ }^{3}$, not to speak of infinite sets and the related measurability problems.

Our axiomatic approach to conditional preferences will be different in two regards. To begin with, we consider a single incomplete preference order, that is a preorder, instead of a family of complete preference orders indexed by the events of the sigma algebra and related to each other by an aggregation condition. Moreover, our approach will take into account additional information only in a local sense: For two given positions there will be some additional information, possibly very detailed, which will allow to rank them, but this information may depend on these two positions and may differ from

\footnotetext{
${ }^{3}$ In the simplest setting one can think about, the binary trees, after only 5 steps you have to consider 4.294.967.296 different preference relations and the corresponding consistent mutual aggregations.
} 
the information needed to rank two other positions. Chapter 4, the first chapter in this second part, provides a set of axioms for our local conditional approach. Although our axioms are only of local nature, an application of ZorN's lemma yields for any two positions a global partition of the set of scenarios into three subsets over which these positions are either ranked or equivalent. In this axiomatic framework for conditional preferences, in principle, could be carried out in full generality. In this thesis, however, we concentrate on the setting of stochastic kernels where it is possible to separate model risk from distributional risk

The following Chapter 5 derives robust representations for conditional preferences. We first focus on the subset of $\mathscr{G}$-measurable stochastic kernels $\mu(\cdot, x)$, in analogy to our discussion of probability distributions in the unconditional case in the first part. In this context, we prove a conditional version of the VON NEUMANN and MORGENSTERN representation for affine conditional preferences. It takes the form

$$
\tilde{u}(\cdot, \mu)=\int u(\cdot, x) \mu(\cdot, d x)
$$

which maps any $\mathscr{G}$-measurable stochastic kernel $\mu$ to a $\mathscr{G}$-measurable random variable $\tilde{u}(\cdot, \mu)$. The conditional utility function $u$ in this representation is continuous and increasing in the second component, and also $\mathscr{G}$-measurable in the first component. In particular, even probability distributions which are independent of the scenario might nevertheless be differently ranked depending on the given scenario. One of the difficulty in the proof of this representation is to show that this utility function which is contingent on the information is still continuous on $\mathbb{R}$ almost surely.

Our next goal is to is to pass from the conditional von NEUMANN and MorgenSTERN representation to a robust representation for conditional variational preferences on general stochastic kernels. In a first step we show that these conditional variational preferences admit a numerical representation of the form

$$
\tilde{U}(\tilde{X})=-\rho\left(\int u(\cdot, x) \tilde{X}(\cdot, d x)\right)
$$

whereby $\tilde{X}$ is now a stochastic kernel, not necessarily $\mathscr{G}$-measurable as above. Here, $\rho$ is a conditional cash additive risk measure on the level of random variables, and $u$ is a conditional utility function just as in the case of the conditional VON NEUMANN and MORGENSTERN representation.

In a second step we express the conditional cash additive risk measure $\rho$ in a robust form. This yields a representation of the conditional variational preferences on stochastic kernels of the form

$$
\tilde{U}(\tilde{X})=-\underset{Q}{\operatorname{ess} \sup _{Q}}\left\{-E_{Q}\left[\int u(\cdot, x) \tilde{X}(\cdot, d x) \mid \mathscr{G}\right]-\alpha(Q)\right\} .
$$




\section{INTRODUCTION}

Here the $Q$ are additive nonnegative set functions normalised to 1 , and $\alpha$ is a $\mathscr{G}$ conditional penalty function weighting the probability models $Q$ according to their respective plausibility given the additional information in $\mathscr{G}$. Under additional regularity assumptions on the preference level, the essential supremum over additive set functions can be restricted to the set of probability measures. Here, we cannot simply apply the standard results on the robust representation of risk measure as in DETLEFSEN AND SCANDOlo [2005], since our reference measure $P$ is only specified on the smaller sigma-algebra $\mathscr{G}$. Instead, we use a separation theorem for locally convex $\mathbb{L}^{0}$-modules of FILIPOVIC ET AL. [2009], which yields the natural conditional analogue to the standard separation arguments in the unconditional case. In addition we have to take special care in defining the conditional expectation appearing in (VIII).

The robust representation (VII) explicits the distinction between the two dimensions of risk: a model risk part in the form of a conditional cash additive risk measure, and a distributional risk related to the ranking of distributions in the sense of VON NEUMAnN and Morgenstern. It is thus natural to ask which axioms for the variational preferences are specifically related to the model risk or to the distributional risk. In other words, which axiom should we strengthen to get a "pure" numerical representation either only in terms of a conditional cash additive risk measures, or only in terms of an expected utility. The answer is the following. Pure distributional risk is characterized by a so called risk independence, that is, if two positions are equivalent, then their convex combination is equivalent to one or the other. Under this assumption the robust representation reduces to

$$
\tilde{U}(\tilde{X})=E_{Q}\left[\int u(\cdot, x) \tilde{X}(\cdot, d x) \mid \mathscr{G}\right],
$$

with one single probability measure $Q$. This is the conditional version of the SAvage representation for stochastic kernels, and it extends the representation (VI) by SKIADAS [1997A] for random variables mentioned previously. On the other hand, pure model risk is characterised by a translation invariance of the preferences: the preference relation between positions remains the same even if those positions are translated in a $\mathscr{G}$-measurable way. In this case, and under an additional technical condition of strict convexity of the preferences, the utility function $u$ reduces to the identity, and the robust representation takes the form

$$
\tilde{U}(\tilde{X})=-\underset{Q}{\operatorname{ess} \sup }\left\{E_{Q}\left[\int \tilde{X}(\cdot, d x) \mid \mathscr{G}\right]-\alpha(Q)\right\} .
$$

\section{Notations and Basic Concepts}

Throughout, the extended real line $[-\infty,+\infty]:=\mathbb{R} \cup\{-\infty,+\infty\}$ is considered with its canonical order and the convention $(+\infty)+(-\infty)=+\infty$. The extended real line 
endowed with the metric $d(x, y):=\arctan (|x-y|)$ is a complete separable metrisable space. A function $f: \mathcal{X} \rightarrow[-\infty,+\infty]$, whereby $\mathcal{X} \subset \mathcal{V}$ is a convex subset of a vector space $\mathcal{V}$, is

- convex if $f(\lambda x+(1-\lambda) y) \leq \lambda f(x)+(1-\lambda) f(y)$ for any $x, y \in \mathcal{V}$ and $\lambda \in] 0,1[$, concave if $-f$ is convex, and affine if $f$ is concave and convex,

- quasiconvex if $f(\lambda x+(1-\lambda) y) \leq \max \{f(x), f(y)\}$ for any $x, y \in \mathcal{V}$ and $\lambda \in$ ]0,1[, quasiconcave if $-f$ is quasiconvex, and quasiaffine if $f$ is quasiconvex and quasiconcave.

A convex function $f: \mathcal{X} \rightarrow[-\infty,+\infty]$ is proper if $f>-\infty$ and $f(x) \in \mathbb{R}$ for some $x \in \mathcal{X}$. A concave function $f: \mathcal{X} \rightarrow \mathbb{R}$ is proper if $-f$ is proper.

If $\mathcal{X}$ is a topological vector space, a function $f: \mathcal{X} \rightarrow[-\infty,+\infty]$ is lower semicontinuous if $\{x \in \mathcal{X} \mid f(x) \leq \alpha\}$ is closed for all $\alpha \in \mathbb{R}$ and upper semicontinuous if $-f$ is lower semicontinuous. The Fenchel-Legendre conjugate $f^{*}$ of a function $f$ is defined as

$$
f^{*}\left(x^{*}\right):=\sup _{x \in \mathcal{X}}\left\{\left\langle x^{*}, x\right\rangle-f(x)\right\}, \quad x^{*} \in \mathcal{X}^{*},
$$

whereby $\mathcal{X}^{*}$ is the topological dual of $\mathcal{X}$ and with the convention that $\sup \emptyset=\inf \mathbb{R}=$ $-\infty$ and $\sup \mathbb{R}=\inf \emptyset=-\infty$.

For any nondecreasing function $f: \mathbb{R} \rightarrow[-\infty,+\infty]$, we denote by $f^{-}$and $f^{+}$the respective unique left- and right-continuous versions of $f$ :

$$
f^{-}(s)=\sup _{t<s} f(s) \quad \text { and } \quad f^{-}(s)=\inf _{t>s} f(s), \quad s \in \mathbb{R},
$$

which satisfy $f^{-} \leq f \leq f^{+}$. Note that $f^{-}$and $f^{+}$only differ on a countable subset of $\mathbb{R}$. 



\section{Acknowledgments}

It is a sincere pleasure to acknowledge the importance of those who accompanied me during this time. My first thanks go to my advisor Hans Föllmer. His inspiring and enlightening approach of finance through mathematics which he lively presented in his lectures or during discussions is the reason why I came back to mathematics after years of absence. I strongly appreciate that he left me the room to mature and follow-through with my ideas and I thank him for is constant encouragement to take things further even in times of severe drawbacks. Never being satisfied with partial explanations or vague statements, he continually challenged my understanding of concepts and decisively contributed to clarify my intuitions. Doing so, he substantially improved the conceptual content and the presentation of both the introduction and the second part of this thesis.

Very special thanks are also due to Michael Kupper. The first part of this thesis is the result of a fruitful collaboration with him. He initiated me to delve into the arcane field of functional analysis and showed me how important it is for the understanding of wide parts of economic theory. His technical mastery and unbelievable perseverance allowed us to tackle highly complex problems, in particular the automatic continuity results for affine risk orders on lotteries. In our hours of discussions we never ran out of new ideas and further developments. It is moreover a great pleasure to work with him and I am very thankful for his very kind friendship.

I also thank my friends Michael Högele and Irina Penner. Especially during our time spent in the same office, I really enjoyed the many interesting discussions with Michael about all conceivable subjects except mathematics. These were a source of inspiration for some ideas in this thesis. Decisive discussions with Irina about the interrelation between time and risk assessment helped me to better understand the dynamical notion of risk. This substantially motivated the conceptual work in the second part of this thesis.

Moreover, I thank my friends and colleagues Claudia Hein, Stefan Ankirchner, Gonçalo dos Reis, Gregor Heyne, Anja Richter whose presence and support contributed to a pleasant atmosphere. Financial support of the DFG through the International Research Training Group "SMCP" and the Berlin Mathematical School are also gratefully acknowledged.

I thank my parents for their love and their unconditional support over the years. I finally gratefully thank my wife Ana for her love and presence. She always keeps me down-to-earth and prevents that I take myself too seriously. 



\section{Part I.}

\section{Risk Preferences and their Robust Representation}





\section{Risk Orders}

\subsection{Risk Orders, Risk Measures and Risk Acceptance Families}

Throughout, we study the risk of positions $x$ in some nonempty space $\mathcal{X}$ where the risk is specified by some total preorder ${ }^{1}$ on $\mathcal{X}$ denoted by $\succcurlyeq$. As usual, the notations $\succ:=\{\succcurlyeq \& \npreceq\}$ and $\sim:=\{\succcurlyeq \& \preccurlyeq\}$ respectively correspond to the antisymmetric and equivalence relation. A numerical representation of a total preorder $\succcurlyeq$ is a mapping $F: \mathcal{X} \rightarrow[-\infty,+\infty]$, such that

$$
x \succcurlyeq y \quad \Longleftrightarrow \quad F(x) \geq F(y)
$$

for any $x, y \in \mathcal{X}$. Further, a total preorder $\succcurlyeq$ is called separable if there exists a countable order dense subset for $\succcurlyeq$, that is, a countable set $\mathcal{Z} \subset \mathcal{X}$ such that $x \succcurlyeq z \succcurlyeq y$ for some $z \in \mathcal{Z}$ whenever $x \succ y$ for any two positions $x, y \in \mathcal{X}$. It is well-known that countable order dense subsets characterize those total preorders which have up to increasing transformations a unique numerical representation as stated in the following proposition.

Proposition 1.1. A total preorder $\succcurlyeq$ has a numerical representation if and only if it is separable. Moreover, a numerical representation of $\succcurlyeq$ is unique up to increasing transformations, that is, for two numerical representations $F, \hat{F}$ of $\succcurlyeq$, there exists an increasing function $h: \operatorname{Im}(F) \rightarrow \operatorname{Im}(\hat{F})$ such that $\hat{F}=h \circ F$.

For a proof of the first assertion we refer to [Föllmer and Schied, 2004, Theorem 2.6] and the uniqueness up to increasing transformation is straightforward.

Our aim is yet to characterize those total preorders which deserve the denomination "risk". As evoked in the introduction, the main properties related to risk perception are diversification and some form of monotonicity. In order to diversify risky position, we need to express convex combinations, thus, $\mathcal{X}$ is from now on a convex subset of a vector space $\mathcal{V}$. As for the monotonicity, we might want a relation to express the fact that some elements are in some sense "better for sure" than others. This relation is expressed by some vector preorder $\triangleq$ on $\mathcal{X}$.

Definition 1.2. A vector preorder $\triangleq$ is the restriction to $\mathcal{X}$ of a binary relation $\unrhd$ defined on the vector space $\mathcal{V} \supset \mathcal{X}$ which is reflexive and transitive and such that $x \unrhd y$ implies $x+z \unrhd y+z$ for any $z \in \mathcal{V}$ and $\lambda x \unrhd \lambda y$ for any $\lambda \geq 0$.

\footnotetext{
${ }^{1} \mathrm{~A}$ total preorder is a transitive and complete binary relation. A binary relation $\succcurlyeq$ on $\mathcal{X}$ is transitive if $x \succcurlyeq y$ and $y \succcurlyeq z$ implies $x \succcurlyeq z$, and is complete if $x \succcurlyeq y$ or $y \succcurlyeq x$ for any $x, y \in \mathcal{X}$.
} 


\section{Risk Orders}

Remark 1.3. For this section we could have considered a general preorder as for $\unrhd$. However, for the study of additional properties in the next sections, we will need a compatibility with the structure of the vector space.

Such a vector order defines in particular a convex cone $\mathcal{K}=\{x \in \mathcal{X} \mid x \triangleq 0\}$ and this cone is such that $x \unrhd y$ exactly when $x-y \in \mathcal{K}$. Note that $\unrhd$ is not necessarily a partial order, and therefore, unlike in [Aliprantis and Border, 2006], the convex cone $\mathcal{K}$ has not to be pointed, that is, $\mathcal{K} \cap-\mathcal{K} \neq\{0\}$. By abuse of language, we simply use the term preorder for vector preoder.

Having introduced the main elements and the general setting, we define the concept of risk orders.

Definition 1.4 (Risk Order). A total preorder $\succcurlyeq$ on $\mathcal{X}$ is a risk order if it is

- quasiconvex: $x \succcurlyeq \lambda x+(1-\lambda) y$ for any $\lambda \in] 0,1[$ whenever $x \succcurlyeq y$.

- monotone: $x \succcurlyeq y$ whenever $y \unrhd x$.

Since we speak about risk and not utility, the relation $x \succcurlyeq y$ has to be read " $x$ is riskier than $y$ ". The quasiconvexity axiom reflects exactly that the diversification between two alternatives keeps the risk below the worst one. Note that it is the quasiconvexity and not the convexity that expresses "diversification does not increase the risk". The monotonicity axiom states that if an alternative is better for sure than another, its risk should then be lower.

In the following, $\mathcal{L}(x)=\{y \in \mathcal{X} \mid x \succcurlyeq y\}$ and $\mathcal{U}(x)=\{y \in \mathcal{X} \mid y \succcurlyeq x\}$ describe the subsets of $\mathcal{X}$ of those elements which are respectively less or more risky than $x \in \mathcal{X}$. Note that the risk order $\succcurlyeq$ is quasiconvex exactly when $\mathcal{L}(x)$ is convex for all $x \in \mathcal{X}$.

Remark 1.5. If the total preorder $\succcurlyeq$ were considered from the utility point of view, $\mathcal{U}(x)$ would be convex for any $x \in \mathcal{X}$. In the literature, the quasiconvexity of a total preorder is often referred to as convexity, e.g. [Jones, 1984, Mas-Colell, 1986, Fishburn, 1988]. We use the denomination quasiconvexity as it exactly corresponds to the quasiconvexity of the corresponding numerical representation.

Note also that the monotonicity concept can be ruled out if the vector preorder $\unrhd$ is trivial, that is, the relation $x \geqslant y$ holds if and only if $x=y$.

The abstractness of the setting agrees with our declared intention to concentrate solely on the structure characterizing the risk as such. This allows us to appreciate and interpret it under different lights depending on the choice of the underlying context. We precise this thereafter with several-though not exhaustive -illustrative settings which will be studied in Chapter 3 .

- Random Variables: In finance, risky positions - equities, credits, derivative products, insurance contracts, portfolios, etc.- -are commonly random variables on some measurable space $(\Omega, \mathscr{F})$. Usually, capital letters $X, Y, \ldots$ are used instead of $x, y, \ldots$ to refer to those risky positions. A possible choice for $\mathcal{X}$ are the sets $\mathbb{L}^{p}:=\mathbb{L}^{p}(\Omega, \mathscr{F}, P)$ of random variables with finite $p$-norm where $P$ is a 
reference probability measure on the $\sigma$-algebra of possible scenarios $\mathscr{F}$. Here, on $\mathbb{L}^{p}$, the canonical preorder is given by the relation "greater than $P$-almost surely". This preorder relation is not always adequate though. Financial investors when facing cumulative wealth streams - stocks, indexes, etc. - might be interested in a relation based on cash flow level because dividends or other derivative instruments could depend on returns. For a fixed time horizon $T>0$, we consider discrete time processes $X=X_{0}, X_{1}, \ldots, X_{T}$ where $X_{t}$ is bounded random variable modelling the cumulative amount of wealth at time $t$. For such a cumulative wealth process, we denote the corresponding cash flow by $\Delta X_{t}=X_{t}-X_{t-1}$ for $t=0, \ldots, T$ with convention $\Delta X_{0}=X_{0}$. The cash flow preorder $X \unrhd Y$ is then given by $\Delta X_{t} \geq \Delta Y_{t} \quad P$-almost surely for any $t=0, \ldots, T$.

- Probability Distributions: Historically, probability distributions, sometimes called lotteries, play an important role in the analysis of risk or preferences in the economic theory. Here also, the tradition sees the use of the notation $\mu, \nu, \ldots$ instead of $x, y, \ldots$ We will consider the set $\mathcal{M}_{1, c}$ of probability distributions with compact support on an open intervall ${ }^{2} I \subset \mathbb{R}$. It spans as a convex set the vector space $c a_{c}$ of bounded signed measures with compact support on $I$. Different orders might be considered on $\mathcal{M}_{1, c}$, such as the first stochastic order defined by $\mu \unrhd \nu$ if $\int u d \mu \geq \int u d \nu$ for any continuous nondecreasing function $u: I \rightarrow \mathbb{R}$. Common also is the second stochastic order which requires additionally to the definition of the first stochastic order that $u$ has to be concave.

- Consumption Streams: To take into account gulps along continuity, Hindy, Huang, and Kreps [1992] proposed to model consumption patterns with nondecreasing right-continuous paths $c:[0,1] \rightarrow[0,+\infty[$ instead of continuous paths. The value $c_{t}$ represents here the cumulative amount of consumption of commodity $c$ up to time $t \in[0,1]$. The commodity space of those consumptions streams will be denoted by $\mathcal{C} \mathcal{S}_{+}:=\mathcal{C} \mathcal{S}_{+}([0,1])$. It is a convex cone which spans the vector space $\mathcal{C S}=\mathcal{C S}([0,1])$ of right-continuous functions with bounded variations. In [Hindy et al., 1992], the preorder is defined as $c^{(1)} \triangleq c^{(2)}$ when $c^{(1)}-c^{(2)} \in \mathcal{C} \mathcal{S}_{+}$ which is analogue to the zeroth stochastic order for the signed measures $c a_{c}$.

- Stochastic Kernels: Since they unify probability distributions and random variables, stochastic kernels are adequate to understand the interrelation between distributional risk and model risk. Used first in economic theory by ANSCOMBE AND Aumann [1963], this setting was adopted by GilboA AND SchmeIdLER [1989] for their maximin expected utility with multiple prior, and further by [Föllmer and Schied, 2004, Maccheroni et al., 2006a, Cerreia-Vioglio et al., 2008b]. Stochastic kernels are probability distributions which are additionally subject to model uncertainty. For instance, let

$$
\tilde{X}(\omega)=\mu_{1} 1_{\left\{\omega=\omega_{1}\right\}}+\mu_{2} 1_{\left\{\omega=\omega_{2}\right\}}+\mu_{3} 1_{\left\{\omega=\omega_{3}\right\}}+\mu_{4} 1_{\left\{\omega=\omega_{4}\right\}}
$$

${ }^{2}$ Other space of probability distribution might be considered. 


\section{Risk Orders}

describes the distribution losses of a long term insurance contract depending on the local temperature distribution. This distribution depends also on the four main greenhouse gas emission scenarios $\omega_{1}, \ldots, \omega_{4}$ given in IPCC [2000] whose occurrence probability is subject to uncertainty. Mathematically, they are measurable mappings ${ }^{3} \tilde{X}: \Omega \rightarrow \mathcal{M}_{1, c}$ where $(\Omega, \mathscr{F}, P)$ is a probability space. This space of stochastic kernels denoted by $\mathcal{S K}$ will be further precised in the Section 3.4. As for the preorder we consider the $P$-almost sure first sochastic order, that is, $\tilde{X} \unrhd \tilde{Y}$ if

$$
\int u(s) \tilde{X}(\omega, d s) \geq \int u(s) \tilde{Y}(\omega, d s), \quad \text { for } P \text {-almost all } \omega \in \Omega,
$$

and any nondecreasing continuous function $u: I \rightarrow \mathbb{R}$.

Remark 1.6. Note that the notion of diversification depends on the underlying setting. A convex combination $\lambda X+(1-\lambda) Y$ of two random variables $X, Y \in \mathbb{L}^{\infty}$ is in terms of diversification crucially different of the convex combination of their respective laws $\lambda \mu+(1-\lambda) \nu$ where $\mu=P_{X}$ and $\nu=P_{Y}$ belongs to $\mathcal{M}_{1, c}$. Indeed, in general holds $P_{\lambda X+(1-\lambda) Y} \neq \lambda P_{X}+(1-\lambda) P_{Y}$.

By Proposition 1.1, a separable risk order can be represented by a numerical representation which from now on is called risk measure and generically denoted by $\rho$. We are going to formulate in Theorem 1.10 a correspondence between risk measures and risk orders. To this end, we need the following autonomous definition of a risk measure.

Definition 1.7 (Risk Measure). A mapping $\rho: \mathcal{X} \rightarrow[-\infty,+\infty]$ is called a risk measure if it is

- quasiconvex: $\rho(\lambda x+(1-\lambda) y) \leq \max \{\rho(x), \rho(y)\}$ for any $x, y \in \mathcal{X}$ and $\lambda \in$ ]0,1[.

- monotone: $\rho(x) \leq \rho(y)$ whenever $x \triangleq y$.

Example 1.8. The certainty equivalent of an expected loss can be considered on the level of probability distributions on $I=] a_{0},+\infty\left[\right.$ for some $a_{0} \in[-\infty,+\infty[$,

$$
\rho(\mu):=l^{-1}\left(\int l(-x) \mu(d x)\right), \quad \mu \in \mathcal{M}_{1, c},
$$

where $l:]-\infty,-a_{0}[\rightarrow \mathbb{R}$ is a loss function, that is, a continuous increasing function with inverse $l^{-1}$ which is also increasing. The function $\rho$ is clearly monotone with respect to the first stochastic order. Further, since any monotone function from $I$ to $\mathbb{R}$

\footnotetext{
${ }^{3}$ Measurable in the $\mathscr{F}-\mathscr{P}\left(\mathcal{M}_{1, c}\right)$ sense where $\mathscr{P}\left(\mathcal{M}_{1, c}\right)$ is the $\sigma$-algebra induced by the mapping $\mu \mapsto \mu(A)$ for any Borel set $A \subset I$.
} 
is automatically quasiaffine, it follows

$$
\begin{aligned}
\rho(\lambda \mu+(1-\lambda) \nu) & =l^{-1}\left(\lambda \int l(-x) \mu(d x)+(1-\lambda) \int l(-x) \nu(d x)\right) \\
& \leq \max \{\rho(\mu), \rho(\nu)\},
\end{aligned}
$$

for any $\mu, \nu \in \mathcal{M}_{1, c}$ and $\left.\lambda \in\right] 0,1[$ and so $\rho$ is a risk measure.

We can as well define the certainty equivalent on the level of random variables

$$
\hat{\rho}(X):= \begin{cases}l^{-1}(E[l(-X)]), & \text { if } X>a_{0} P \text {-almost surely } \\ +\infty, & \text { else }\end{cases}
$$

for any $X \in \mathbb{L}^{\infty}$, whereby $l^{-1}(+\infty)=+\infty$ by convention. Even if $\hat{\rho}(X)=\rho\left(P_{X}\right)$, according to the previous Remark 1.6, we need further assumptions on $l$ such that $\hat{\rho}$ is a risk measure. We assume here that $l$ is additionally convex. The functional $\hat{\rho}$ is obviously monotone with respect to the relation "greater than $P$-almost surely". Since $l$ is convex and $l^{-1}$ quasiaffine, it follows

$$
\begin{aligned}
l^{-1}(E[l(-\lambda X-(1-\lambda) Y)]) & \leq l^{-1}(\lambda E[l(-X)]+(1-\lambda) E[l(-Y)]) \\
& \leq \max \left\{l^{-1}(E[l(-X)]), l^{-1}(E[l(-Y)])\right\}
\end{aligned}
$$

for any $\lambda \in] 0,1[$, showing that $\hat{\rho}$ is a risk measure. A study of the robust representation of this risk measure will be given in Section 3.1, Example 3.7, where some typical utility functions will be treated.

Before moving onto the theorem stating the relation between risk measures and risk orders, we introduce another concept crucial for the further understanding of this paper. Given a risk measure $\rho$, for any risk level $m \in \mathbb{R}$, we define the risk acceptance set of level $m$ as the subset $\mathcal{A}_{\rho}^{m} \subset \mathcal{X}$ of those positions having a risk smaller than $m$, that is

$$
\mathcal{A}_{\rho}^{m}=\{x \in \mathcal{X} \mid \rho(x) \leq m\}, \quad m \in \mathbb{R} .
$$

We call $\mathcal{A}_{\rho}=\left(\mathcal{A}_{\rho}^{m}\right)_{m \in \mathbb{R}}$ the risk acceptance family associated to $\rho$. Here again, the risk acceptance family carries the specificities of the risk measure. In Theorem 1.10 we will state a one-to-one relation between risk measures and risk acceptance family satisfying the following adequate assumptions.

Definition 1.9 (Risk Acceptance Family). A family $\mathcal{A}=\left(\mathcal{A}^{m}\right)_{m \in \mathbb{R}}$ of sets $\mathcal{A}^{m} \subset$ $\mathcal{X}$ is a risk acceptance family if it is

- convex: $\mathcal{A}^{m}$ is a convex subset of $\mathcal{X}$ for any $m \in \mathbb{R}$.

- monotone: in the following two meanings 


\section{Risk Orders}

(i) $\mathcal{A}^{m} \subset \mathcal{A}^{n}$ for any risk acceptance levels $m \leq n$,

(ii) $x \in \mathcal{A}^{m}$ and $y \unrhd x$ implies $y \in \mathcal{A}^{m}$.

- right-continuous: $\mathcal{A}^{m}=\bigcap_{n>m} \mathcal{A}^{n}$ for any $m \in \mathbb{R}$.

This concept is not only a powerful tool to prove structural properties of risk measures; it can be meaningful to start with to model specific economical features of risk: Numéraire uncertainty or liquidity costs for instance, see Examples 1.29 and 1.30. Be aware that the right-continuity condition for the risk acceptance family is not of topological nature.

On the level of the risk acceptance family, the monotonicity axiom states that any position in a given risk acceptance set level belongs to any higher risk acceptance set, and that it is compatible with the preorder $\unrhd$. The convexity axiom expresses that any diversification of risky positions in some risk acceptance set stays within this risk acceptance set. Finally, the right-continuity is needed to ensure the one-to-one correspondence between risk orders, risk measures, and risk acceptance families as stated in the following theorem.

Theorem 1.10. Given a separable risk order $\succcurlyeq$, any corresponding numerical representation $\rho_{\succcurlyeq}: \mathcal{X} \rightarrow[-\infty,+\infty]$ is a risk measure. Conversely, any risk measure $\rho: \mathcal{X} \rightarrow[-\infty,+\infty]$ defines a risk order $\succcurlyeq_{\rho}$ through

$$
x \succcurlyeq_{\rho} y \quad \Longleftrightarrow \quad \rho(x) \geq \rho(y)
$$

Moreover, $\succcurlyeq_{\rho_{\succcurlyeq}}=\succcurlyeq$ and $\rho_{\succcurlyeq}=h \circ \rho$ for some increasing transformation $h$.

For a risk measure $\rho$, the family $\mathcal{A}_{\rho}$ given by

$$
\mathcal{A}_{\rho}^{m}:=\{x \in \mathcal{X} \mid \rho(x) \leq m\}, \quad m \in \mathbb{R},
$$

is a risk acceptance family. Conversely, for any risk acceptance family $\mathcal{A}$, the functional $\rho_{\mathcal{A}}$ given by

$$
\rho_{\mathcal{A}}(x):=\inf \left\{m \in \mathbb{R} \mid x \in \mathcal{A}^{m}\right\}, \quad x \in \mathcal{X},
$$

defines a risk measure. Moreover, $\rho_{\mathcal{A}_{\rho}}=\rho$ and $\mathcal{A}_{\rho_{\mathcal{A}}}=\mathcal{A}$.

Proof. It is straightforward to check that $\succcurlyeq$ is a risk order if and only if $\rho_{\succcurlyeq}$ is a risk measure, that $\succcurlyeq_{\rho_{\succcurlyeq}}=\succcurlyeq$, and that $\rho$ and $\rho_{\succcurlyeq \rho}$ coincide up to an increasing transformation. It remains to show the one-to-one relation between risk measures and risk acceptance families.

Step 1. Let $\rho$ be a risk measure with corresponding level sets

$$
\mathcal{A}_{\rho}^{m}=\{x \in \mathcal{X} \mid \rho(x) \leq m\}, \quad m \in \mathbb{R} .
$$

From this definition, $\mathcal{A}_{\rho}^{m} \subset \mathcal{A}_{\rho}^{n}$ for any $m \leq n$ which together with the monotonicity of $\rho$ implies the monotonicity of $\mathcal{A}_{\rho}$. Since level sets of quasiconvex functionals are convex it follows that $\mathcal{A}_{\rho}$ is convex. Obviously, $\mathcal{A}_{\rho}^{m} \subset \bigcap_{n>m} \mathcal{A}_{\rho}^{n}$ for any $m \in \mathbb{R}$, and conversely, 
if $x \in \bigcap_{n>m} \mathcal{A}_{\rho}^{n}$, then $\rho(x) \leq n$ for any $n>m$ implying $\rho(x) \leq m$ and therefore $x \in \mathcal{A}_{\rho}^{m}$, showing the right-continuity. And so, $\mathcal{A}_{\rho}$ is a risk acceptance family.

$S$ tep 2. Conversely, let $\mathcal{A}=\left(\mathcal{A}^{m}\right)_{m \in \mathbb{R}}$ be a risk acceptance family and let $\rho_{\mathcal{A}}$ be the functional defined as

$$
\rho_{\mathcal{A}}(x)=\inf \left\{m \in \mathbb{R} \mid x \in \mathcal{A}^{m}\right\}, \quad x \in \mathcal{X} .
$$

As for the monotonicity, consider $x \triangleq y$ and those ${ }^{4} m \in \mathbb{R}$ where $y \in \mathcal{A}^{m}$. From the monotonicity of $\mathcal{A}$ follows $x \in \mathcal{A}^{m}$. Hence, $m \geq \rho_{\mathcal{A}}(x)$ for any $m \in \mathbb{R}$ with $y \in \mathcal{A}^{m}$ and therefore $\rho_{\mathcal{A}}(y) \geq \rho_{\mathcal{A}}(x)$ showing the monotony of $\rho_{\mathcal{A}}$. Concerning the quasiconvexity, let $x, y \in \mathcal{X}$ with $^{5} \rho_{\mathcal{A}}(x) \geq \rho_{\mathcal{A}}(y)$, pick some $\left.\lambda \in\right] 0,1[$ and consider those $m \in \mathbb{R}$ such that $x \in \mathcal{A}^{m}$. The monotonicity implies that also $y \in \mathcal{A}^{m}$ and by convexity of $\mathcal{A}^{m}$ it follows $\lambda x+(1-\lambda) y \in \mathcal{A}^{m}$. This implies that $\rho_{\mathcal{A}}(\lambda x+(1-\lambda) y) \leq m$ for any $m \in \mathbb{R}$ where $x \in \mathcal{A}^{m}$ and therefore $\rho_{\mathcal{A}}(\lambda x+(1-\lambda) y) \leq \rho_{\mathcal{A}}(x)=\max \left\{\rho_{\mathcal{A}}(x), \rho_{\mathcal{A}}(y)\right\}$. Hence, $\rho_{\mathcal{A}}$ is a risk measure.

$S$ tep3. Let $\rho$ be a risk measure. In view of the first and second step, $\rho_{\mathcal{A}_{\rho}}$ is also a risk measure. If $x \in \mathcal{X}$ is such that $\rho(x)=+\infty$, then it is unacceptable at any level of risk for $\mathcal{A}_{\rho}$, and therefore $\rho_{\mathcal{A}_{\rho}}(x)=+\infty$. The same argumentation holds for those $x \in \mathcal{X}$ satisfying $\rho(x)=-\infty$. If $\rho(x) \in \mathbb{R}$, by definition, $x \in \mathcal{A}_{\rho}^{\rho(x)}$, hence $\rho_{\mathcal{A}_{\rho}}(x) \leq \rho(x)$. On the other hand, also by definition, $x \notin \mathcal{A}_{\rho}^{n}$ for any $n<\rho(x)$, henceforth $\rho_{\mathcal{A}_{\rho}}(x) \geq \rho(x)$ and so $\rho=\rho_{\mathcal{A}}$.

Let $\mathcal{A}$ be a risk acceptance family. From the first and second step, $\mathcal{A}_{\rho_{\mathcal{A}}}$ is also a risk acceptance family. By definition, if $x \in \mathcal{A}^{m}$ for some $m \in \mathbb{R}$, it follows $\rho_{\mathcal{A}}(x) \leq m$ yielding $x \in \mathcal{A}_{\rho_{\mathcal{A}}}^{m}$. Conversely, $x \in \mathcal{A}_{\rho_{\mathcal{A}}}^{m}$ implies $\rho_{\mathcal{A}}(x) \leq m$ which in view of (1.7) yields $x \in \mathcal{A}^{n}$ for any $n>m$. From the right-continuity of $\mathcal{A}$, follows $x \in \bigcap_{n>m} \mathcal{A}^{n}=\mathcal{A}^{m}$ and so $\mathcal{A}=\mathcal{A}_{\rho_{\mathcal{A}}}$.

The idea of expressing the numerical representation of a total preorder by means of a family of acceptance sets as in (1.7) was recently used in other studies. For instance, CHERNy AND MADAN [2009] characterize a class of performance measures built upon a specific family of acceptance sets ${ }^{6}$. Further, BROWN ET AL. [2009] represent a type of prospective preferences also by means of acceptance sets which are however not necessarily convex. In their setting, the acceptance sets are convex up to a certain level of risk and then, it is the complement of the acceptance sets which is convex.

Remark 1.11. In the proof of Theorem 1.10, it turns out that the right-continuity condition for the risk acceptance family $\mathcal{A}$ is not necessary for $\rho_{\mathcal{A}}$ to be a risk measure. Nevertheless, it plays a crucial role in the relation $\mathcal{A}_{\rho_{\mathcal{A}}}=\mathcal{A}$. Indeed, consider on $\mathcal{X}=\mathbb{R}$ the family of real sets $\left.\mathcal{A}^{m}=\right]-m,+\infty$ [. This family is monotone and convex but fails to be right-continuous because $\mathcal{A}^{m} \neq\left[-m,+\infty\left[=\bigcap_{n>m}\right]-n,+\infty[\right.$. An immediate

\footnotetext{
${ }^{4}$ In case where there are no such $m$, i.e. $y$ is unacceptable at any level of risk is trivial as $\rho_{\mathcal{A}}(y)=+\infty$.

${ }^{5}$ Here again, the case where $x$ is unacceptable at any level of risk is obvious.

${ }^{6}$ The risk acceptance family corresponds to the acceptance sets of a family of coherent monetary risk measures as introduced in Section 1.4 thereafter.
} 


\section{Risk Orders}

computation shows that $\rho_{\mathcal{A}}(x)=-x$, hence $\mathcal{A}_{\rho_{\mathcal{A}}}^{m}=[-m,+\infty[$ for any $m \in \mathbb{R}$ and thus $\mathcal{A} \neq \mathcal{A}_{\rho_{\mathcal{A}}}$.

For notational convenience, as soon as no misunderstanding threaten, we drop the index references associated to the risk order, the risk measure or the risk acceptance family when it concerns their respective relations, that is, $\mathcal{A}$ instead of $\mathcal{A}_{\rho}, \succcurlyeq$ instead of $\succcurlyeq_{\rho}$ or $\rho$ instead of $\rho_{\succcurlyeq}$ or $\rho_{\mathcal{A}}$.

To illustrate the previous theorem, we consider two, somehow similar, families of risk measures.

Example 1.12. Introduced by Föllmer AND SCHIED [2002], the shortfall risk measure is of additive nature and given by

$$
\rho(X):=\inf \left\{s \in \mathbb{R} \mid E[l(-X-s)] \leq c_{0}\right\}, \quad X \in \mathbb{L}^{\infty},
$$

where $E[l(-X)]$ is the expected loss of the position $X$ according to a so called loss function $l: \mathbb{R} \rightarrow]-\infty,+\infty]$, that is, a lower semicontinuous convex function increasing on its domain such that $l\left(s_{0}\right)<+\infty$ for some $s_{0}>0$. This risk measure accounts for the minimal amount of money which added to the position $X$ pulls its expected loss below a given threshold $c_{0}$ in the range of $l(] 0,+\infty[)$. From the strict monotonicity and the lower semicontinuity of the loss function $l$ holds

$$
\begin{aligned}
\mathcal{A}^{m}=\left\{X \in \mathbb{L}^{\infty} \mid \rho(X) \leq m\right\} & =\left\{X \in \mathbb{L}^{\infty} \mid E[l(-X-\rho(X))] \geq E[l(-X-m)]\right\} \\
& =\left\{X \in \mathbb{L}^{\infty} \mid c_{0} \geq E[l(-X-m)]\right\}
\end{aligned}
$$

for any risk level $m \in \mathbb{R}$. Since $X \mapsto E[l(-X-m)]$ is convex and monotone, we deduce that $\mathcal{A}_{\rho}$ is a risk acceptance family and therefore, by way of Theorem 1.10, $\rho$ is a risk measure.

Example 1.13. First introduced by Aumann And Serrano [2008] in the exponential case and extended to the logarithmic case by Foster AND HART [FORTHCOMING], the economic index of riskiness is similar to the expected shortfall but of multiplicative nature fitting particularly well for returns. It can be generalized and interpreted as follows ${ }^{7}$. We first define

$$
\lambda(X)=\sup \left\{\lambda>0 \mid E[l(-\lambda X)] \leq c_{0}\right\}, \quad E[X] \geq 0,
$$

which represents the maximal exposure to a position $X$ provided that the expected loss remains below an acceptable level $c_{0}$ in the range of $l(] 0+\infty[)$. Here, $l$ is again a loss function, that is, a lower semicontinuous convex function $l: \mathbb{R} \rightarrow]-\infty,+\infty]$, increasing on its domain such that $l\left(s_{0}\right)<+\infty$ for some $s_{0}>0$. This loss function fulfills in addition the growth condition $\lim _{x \rightarrow+\infty} l(x) / x=+\infty$. Due to the monotonicity

\footnotetext{
${ }^{7}$ In [Aumann and Serrano, 2008, Foster and Hart, forthcoming], the economic index of riskiness is a positive homogeneous functional measuring whether gambles are rejected at a given level of wealth. This viewpoint rather corresponds to the representation (1.9).
} 
and convexity of $l$ this condition insures that expected losses are inflated more than gains since for any $X \in \mathbb{L}^{\infty}$ taking negative values on a set of positive probability, $E[l(-\lambda X)] \rightarrow+\infty$ for $\lambda \rightarrow+\infty$. The economic index of riskiness is then defined as

$$
\rho(X):=\left\{\begin{array}{ll}
1 / \lambda(X) & \text { if } E[X] \geq 0 \\
+\infty, & \text { if } E[X]<0
\end{array}, \quad X \in \mathbb{L}^{\infty},\right.
$$

with the usual convention that $-1 / 0=-\infty$ and $-1 /+\infty=0$. A simple computation yields an equivalent formulation

$$
\rho(X):=\left\{\begin{array}{ll}
\inf \left\{t>0 \mid E[u(X / t)] \geq-c_{0}\right\}, & \text { if } E[X] \geq 0 \\
+\infty, & \text { if } E[X]<0
\end{array}, \quad X \in \mathbb{L}^{\infty}\right.
$$

whereby $u(s):=-l(-s)$, which is concave and increasing on its domain. Given a risk level $^{8} m>0$ holds

$$
\begin{aligned}
\mathcal{A}^{m} & =\left\{X \in \mathbb{L}^{\infty} \mid \lambda(X) \geq 1 / m\right\} \\
& =\left\{X \in \mathbb{L}^{\infty} \mid E[l(-\lambda(X) X)] \geq E[l(-X / m)]\right\} \\
& =\left\{X \in \mathbb{L}^{\infty} \mid c_{0} \geq E[l(-X / m)]\right\},
\end{aligned}
$$

where the second equality holds since $E[l(-\lambda(X) X)] \geq E[l(-\lambda X)]$ if and only if $\lambda(X) \geq \lambda \geq 0$. Indeed, the right derivative of $\lambda \mapsto E[l(-\lambda X)]$ is $E\left[-X l^{\prime-}(-\lambda X)\right]$ which at 0 equals to $l^{\prime-}(0) E[-X] \leq 0$. Furthermore, $\lambda \mapsto E[l(-\lambda X)]$ is convex, $l(0)<c_{0}$ and $E[l(-\lambda X)] \rightarrow+\infty$. This means that $\lambda \mapsto E[l(-\lambda X)]$ starts from 0 at $l(0)$, decreases first and then starts to increase before attaining, in reason of the lower semicontinuity of $l$, the level $c_{0}>l(0)$. The third equality holds because $E[l(-\lambda(X) X)]=c_{0}$ in reason of the lower semicontinuity of $l$. From the convexity and the monotonicity of $X \mapsto E[l(-\lambda X)]$ is $\mathcal{A}$ convex and monotone and thus a risk acceptance family. Therefore, applying Theorem 1.10, the economic index of riskiness is a risk measure.

The functions used in [Aumann and Serrano, 2008, Foster and Hart, forthcoming], respectively correspond to $l(s)=-u(-s)=e^{s}-1$ and $l(s)=-\ln (1-s)$ which are both loss functions fulfilling the required growth conditions. Here again, a computation of the robust representation of the economic index of riskiness will be given in Section 3.1, Example 3.8.

Remark 1.14. Theorem 1.10 ensures that as soon as of one of these objects - risk order, risk measure or risk acceptance family - is given, the other two are simultaneously precised. The notion of quasiconvexity and monotonicity are therefore global features; as soon as one numerical representation has these properties, they are automatically shared by the corresponding risk order and the whole class of its numerical representations and vice versa. In the following subsections, we will study additional properties of the nu-

${ }^{8}$ Clearly, for any $m<0$ holds $\mathcal{A}_{\rho}^{m}=\emptyset$, and $\mathcal{A}_{\rho}^{0}=\mathbb{L}_{+}^{\infty}$ which are both convex. 


\section{Risk Orders}

merical representation, such as convexity, affinity, or cash additivity amongst others. Unlike quasiconvexity and monotonicity, they do not hold for the entire class of numerical representations of the corresponding risk order. These properties are in this sense local. Concerning these local properties, we later speak of a convex - respectively affine, cash additive, etc.- - risk order when there exists at least one numerical representation having this property.

\subsection{Convexity, Positive Homogeneity, Scaling Invariance}

Having introduced the general concept of risk, we here address now the consequences of additional assumptions such as convexity, positive homogeneity or scaling invariance. As mentioned in the Remark 1.14 above, these properties are no longer global. We define those properties for the risk measures and state in a subsequent proposition their impact on the corresponding risk order and risk acceptance family.

Definition 1.15. A risk measure $\rho: \mathcal{X} \rightarrow[-\infty,+\infty]$ is

- convex if $\rho(\lambda x+(1-\lambda) y) \leq \lambda \rho(x)+(1-\lambda) \rho(y)$ for any $x, y \in \mathcal{X}$ and $\lambda \in] 0,1[$.

- positive homogeneous if $\rho(\lambda x)=\lambda \rho(x)$ for any $x, y \in \mathcal{X}$ and $\lambda>0$.

- scaling invariant if $\rho(\lambda x)=\rho(x)$ for any $x, y \in \mathcal{X}$ and $\lambda>0$.

The notions of positive homogeneity and scaling invariance require in addition that $\mathcal{X}$ is at least a convex cone.

Proposition 1.16. For a risk measure $\rho$, the corresponding risk order $\succcurlyeq$ and the corresponding risk acceptance family $\mathcal{A}$ hold:

(i) $\rho$ is a convex risk measure if and only if $\mathcal{A}$ is level convex, that is, $\lambda \mathcal{A}^{m}+$ $(1-\lambda) \mathcal{A}^{m^{\prime}} \subset \mathcal{A}^{\lambda m+(1-\lambda) m^{\prime}}$ for any $m, m^{\prime} \in \mathbb{R}$ and $\left.\lambda \in\right] 0,1[$.

(ii) $\rho$ is positive homogeneous if and only if $\mathcal{A}$ is positive homogeneous, that is, $\lambda \mathcal{A}^{m}=$ $\mathcal{A}^{\lambda m}$ for any $m \in \mathbb{R}$ and $\lambda>0$.

In that case, the related risk order satisfies $\lambda \mathcal{L}(x)=\mathcal{L}(\lambda x)$ for any $x \in \mathcal{X}$ and $\lambda>0$.

(iii) $\rho$ is scaling invariant if and only if $\mathcal{A}$ is scaling invariant, that is, $\lambda \mathcal{A}^{m}=\mathcal{A}^{m}$ for any $m \in \mathbb{R}$ and $\lambda>0$.

In that case, the related risk order satisfies $\lambda \mathcal{L}(x)=\mathcal{L}(x)$ for any $x \in \mathcal{X}$ and $\lambda>0$.

Proof. (i): Suppose that $\rho$ is convex. Take $\lambda \in] 0,1\left[\right.$ and some reals $m, m^{\prime}$. Any element of $\lambda \mathcal{A}^{m}+(1-\lambda) \mathcal{A}^{m^{\prime}}$ can be written as $\lambda x+(1-\lambda) y$ for $x \in \mathcal{A}^{m}$ and $y \in \mathcal{A}^{m^{\prime}}$. In particular, both positions have a risk smaller than $+\infty$. The convexity implies

$$
\rho(\lambda x+(1-\lambda) y) \leq \lambda \rho(x)+(1-\lambda) \rho(y) \leq \lambda m+(1-\lambda) m^{\prime},
$$


showing that, $\lambda x+(1-\lambda) y \in \mathcal{A}^{\lambda m+(1-\lambda) m^{\prime}}$. Conversely, by Theorem 1.10, for $m, m^{\prime} \in$ $\mathbb{R}$ such that $x \in \mathcal{A}^{m}$ and $y \in \mathcal{A}^{m^{\prime}}$, it follows

$$
\begin{aligned}
\rho(\lambda x+(1-\lambda) y) & =\inf \left\{n \in \mathbb{R} \mid \lambda x+(1-\lambda) y \in \mathcal{A}^{n}\right\} \\
& =\inf _{n, n^{\prime} \in \mathbb{R}}\left\{\lambda n+(1-\lambda) n^{\prime} \mid \lambda x+(1-\lambda) y \in \mathcal{A}^{\lambda n+(1-\lambda) n^{\prime}}\right\} \\
& \leq \inf _{n, n^{\prime} \in \mathbb{R}}\left\{\lambda n+(1-\lambda) n^{\prime} \mid \lambda x+(1-\lambda) y \in \lambda \mathcal{A}^{n}+(1-\lambda) \mathcal{A}^{n^{\prime}}\right\} \\
& \leq \lambda m+(1-\lambda) m^{\prime},
\end{aligned}
$$

showing that

$$
\rho(\lambda x+(1-\lambda) y) \leq \lambda \rho(x)+(1-\lambda) \rho(y) .
$$

The cases where $\rho(x)= \pm \infty$ are obvious.

(ii): Suppose that $\rho$ is coherent. Take $\lambda>0$ and some $m \in \mathbb{R}$. Then, $x \in \mathcal{A}^{\lambda m}$ if and only if $\rho(x) / \lambda \leq m$ if and only if $\rho(x / \lambda) \leq m$ if and only if $x \in \lambda \mathcal{A}^{m}$, i.e., $\lambda \mathcal{A}^{m}=\mathcal{A}^{\lambda m}$. Conversely, Theorem 1.10 yields

$$
\rho(\lambda x)=\inf \left\{m \in \mathbb{R} \mid \lambda x \in \mathcal{A}^{m}\right\}=\inf \left\{m \in \mathbb{R} \mid x \in \mathcal{A}^{m / \lambda}\right\}=\lambda \rho(x) .
$$

Consider now a coherent risk measure $\rho$ and its acceptance set $\mathcal{A}$. Furthermore, since $\mathcal{L}(x)=\mathcal{A}^{\rho(x)}$ it follows

$$
\lambda \mathcal{L}(x)=\lambda \mathcal{A}^{\rho(x)}=\mathcal{A}^{\lambda \rho(x)}=\mathcal{A}^{\rho(\lambda x)}=\mathcal{L}(\lambda x) .
$$

(iii): The proof is analogous to the proof of (ii).

Example 1.17. The celebrated expected utility for random variables introduced by SAVAGE [1972] is given by

$$
\rho(X):=E_{Q}[l(-X)], \quad X \in \mathbb{L}^{\infty},
$$

for some probability measure $Q$ absolutely continuous with respect to $P$ and a continuous function $l: \mathbb{R} \rightarrow \mathbb{R}$. It is an example of a convex risk measure if $l$ is nondecreasing and convex.

The Sharpe Ratio introduced by SHARPE [1964] is given by

$$
\rho(X):=\left\{\begin{array}{ll}
-\frac{E[X]}{\sqrt{E\left[X^{2}-E[X]^{2}\right]}} & \text { if } E[X]>0 \\
0 & \text { else }
\end{array}, \quad X \in \mathbb{L}^{\infty},\right.
$$

with convention that $s / 0=-\infty$ for $s<0$, is quasiconvex and scaling invariant. Even if it is not monotone for the standard relation "greater than $P$-almost surely", it is still a scaling invariant risk measure with respect to the trivial preorder. For several examples of monotone alternatives to the Sharpe Ratio in the context of performance measures 


\section{Risk Orders}

we refer to [Cherny and Madan, 2009].

\subsection{Affine Risk Orders}

From the beginning of utility theory, affine structures were present and source of many interpretations and extensions in the context of preferences and risk. We consequently devote this subsection to this case.

Definition 1.18. A risk measure $\rho: \mathcal{X} \rightarrow[-\infty,+\infty]$ is affine if it takes values in $\mathbb{R}$ and

$$
\rho(\lambda x+(1-\lambda) y)=\lambda \rho(x)+(1-\lambda) \rho(y),
$$

for any $x, y \in \mathcal{X}$ and $\lambda \in] 0,1[$.

As expressed in Remark 1.14, a risk order $\succcurlyeq$ is affine if there exists at least one numerical representation $\rho$ which is an affine risk measure. It is well-known that the independence and Archimedian properties are the necessary and sufficient conditions to guarantee affinity.

Proposition 1.19. A risk order $\succcurlyeq$ is affine if and only if it fulfills the following properties

- Independence: $x \succ y$ implies $\lambda x+(1-\lambda) z \succ \lambda y+(1-\lambda) z$ for any $z \in \mathcal{X}$ and $\lambda \in] 0,1[$.

- Archimedian: $x \succ z \succ y$ implies the existence of $\lambda, \beta \in] 0,1[$ such that

$$
\lambda x+(1-\lambda) y \succ z \succ \beta x+(1-\beta) y .
$$

In that case, $\succcurlyeq$ is separable and the corresponding affine risk measure $\rho$ fulfills

$$
\rho(\lambda x+(1-\lambda) y)=\lambda \rho(x)+(1-\lambda) \rho(y),
$$

for any $x, y \in \mathcal{X}$ and $\lambda \in \mathbb{R}$ such that $\lambda x+(1-\lambda) y \in \mathcal{X}$. It is moreover unique up to strict positive affine transformations in the class of affine risk measures.

Proof. The proof that there exists a numerical representation $\rho: \mathcal{X} \rightarrow \mathbb{R}$ such that

$$
\rho(\lambda x+(1-\lambda) y)=\lambda \rho(x)+(1-\lambda) \rho(y), \quad \text { for any } \lambda \in] 0,1[
$$

and that it is unique up to a strict positive affine transformation is a straightforward adaptation of standard results, [see Föllmer and Schied, 2004, Chapter 2.2].

Let us show that relation (1.14) also holds for those $\lambda \in \mathbb{R}$ for which $\lambda x+(1-\lambda) y \in \mathcal{X}$. If $\lambda \geq 1$, pick $r \in] 0,1[$ such that $r \lambda \in] 0,1[$. On one hand,

$$
\rho(r(\lambda x+(1-\lambda) y)+(1-r) y)=r \rho(\lambda x+(1-\lambda) y)+(1-r) \rho(y),
$$


On the other hand,

$$
\begin{array}{r}
\rho(r(\lambda x+(1-\lambda) y)+(1-r) y)=\rho(\lambda r x+(1-\lambda r) y)=\lambda r \rho(x)+(1-\lambda r) \rho(y) \\
=r(\lambda \rho(x)+(1-\lambda) \rho(y))+(1-r) \rho(y) .
\end{array}
$$

Both relations imply $\rho(\lambda x+(1-\lambda) y)=\lambda \rho(x)+(1-\lambda) \rho(y)$. The same argumentation with $1-\lambda$ instead of $\lambda$ yields the desired result for $\lambda \leq 0$.

Example 1.20. The SAVAGE representation given by relation (1.10) and denoted by $\rho$ is not affine on the level of random variables, unless $u$ were affine. However, since it is law invariant, it can also be considered on the level of probability distribution by the identification $Q_{X}=\mu \in \mathcal{M}_{1, c}$ through

$$
\tilde{\rho}(\mu):=\int l(-x) \mu(d x), \quad \mu \in \mathcal{M}_{1, c} .
$$

This corresponds to the representation of VON NEUMANn AND Morgenstern [1947]. Obviously, $\tilde{\rho}\left(P_{X}\right)=\rho(X)$. On the level of probability distributions, it is yet an affine risk measure - monotone with respect to the first stochastic order if $u$ is nondecreasing. $\diamond$

\subsection{Monetary Risk Orders}

Especially for financial applications, it is sometimes meaningful to express the notion of risk in a monetary sense. To do so, we introduce this monetary notion in the form of a special direction $\pi \in \mathcal{X}$ satisfying $\pi \unrhd 0$. In the financial setting where $\mathcal{X}=\mathbb{L}^{p}$, the vector $\pi$ can be viewed as a numéraire or a risk free bank account, for instance $\pi=1+r$ for some interest rate $r>-1$. Throughout this section, we assume that $\mathcal{X}$ is a vector space. We start with the notion of cash additivity.

Definition 1.21. A risk measure $\rho: \mathcal{X} \rightarrow[-\infty,+\infty]$ is cash additive if

$$
\rho(x+m \pi)=\rho(x)-m,
$$

for any $m \in \mathbb{R}$.

The cash additivity expresses that $\rho(x)$ is precisely the minimal amount of money which has to be reserved on the risk free bank account $\pi$ to pull the risk of the position $x$ under the level 0 as $\rho(x+\rho(x) \pi)=\rho(x)-\rho(x)=0$ and the monotonicity implies $\rho(x+m \pi) \leq 0$ for any $m \geq \rho(x)$.

An axiomatic approach for the concept of a cash additive risk measure has first been introduced by Artzner, Delbaen, EBer, AND HeAth [1999] in terms of coherent cash additive monetary risk measures, that is, in our context, positive homogeneous cash additive risk measures. By Proposition 1.24 below, cash additive risk measures are automatically convex. Thus, the positive homogeneity implies that coherent risk 


\section{Risk Orders}

measures are subadditive, that is, $\rho(x+y) \leq \rho(x)+\rho(y)$. Föllmer AND SCHIED [2002], Frittelli and Rosazza Gianin [2002], Heath [Paris 2000] generalized this concept to convex cash additive risk measures which in our terminology corresponds to cash additive risk measures, that are automatically convex by way of Proposition 1.24 below.

Here again, cash additivity is a property that does not hold for all numerical representations of the corresponding risk order $\succcurlyeq$. Hence, as mentioned in Remark 1.14, a risk order will be called cash additive if it has at least one such numerical representation. As for affine risk orders, we give hereafter some necessary and sufficient conditions for which the risk order is cash additive.

Theorem 1.22. A risk order $\succcurlyeq$ is cash additive if and only if the following two conditions hold

(i) for any $x \in \mathcal{X}$ such that $y \succ x \succ z$ for some $y, z \in \mathcal{X}$ there exists a unique $m \in \mathbb{R}$ which satisfies $x \sim m \pi$;

(ii) $x \succcurlyeq y$ implies $x+m \pi \succcurlyeq y+m \pi$ for any $m \in \mathbb{R}$.

Proof. In case $\rho$ is a cash additive risk measure which represents $\succcurlyeq$, the cash additivity property clearly implies the properties $(i)$ and $(i i)$.

Conversely, let $\mathcal{B}:=\{x \in \mathcal{X} \mid y \succ x \succ z$ for some $y, z \in \mathcal{X}\}$. There exist at most $x_{1}, x_{2} \in \mathcal{X}$ with $x_{2} \succcurlyeq x_{1}$ such that either $x \sim x_{1}, x \sim x_{2}$ or $x \in \mathcal{B}$ for all $x \in \mathcal{X}$. Conditions (i) and (ii) imply that the mapping

$$
\rho(x):=\left\{\begin{array}{ll}
-\infty & \text { if } x \sim x_{1} \\
-m & \text { if } x \in \mathcal{B}, x \sim m \pi \\
+\infty & \text { if } x \sim x_{2}
\end{array},\right.
$$

defines a cash additive risk measure which represents $\succcurlyeq$.

Furthermore, cash additive risk measures are fully characterized by the special shape of their risk acceptance family.

Proposition 1.23. A risk measure $\rho$ is cash additive if and only if the related risk acceptance family $\mathcal{A}$ satisfies

$$
\mathcal{A}^{0}=\mathcal{A}^{m}+m \pi, \quad \text { for all } m \in \mathbb{R} .
$$

Proof. Let $\rho$ be a cash additive risk measure and fix some $m \in \mathbb{R}$. The respective risk acceptance family $\mathcal{A}$ satisfies

$$
\mathcal{A}^{m}=\{x \in \mathcal{X} \mid \rho(x) \leq m\}=\{x \in \mathcal{X} \mid \rho(x+m \pi) \leq 0\}=\mathcal{A}^{0}-m \pi,
$$

and therefore fulfills the condition (1.16). 
Conversely, let $\mathcal{A}$ be risk acceptance family satisfying relation (1.16). The cash additivity for the related risk measure $\rho$ follows from

$$
\rho(x+m \pi)=\inf \left\{m^{\prime} \mid x+\left(m+m^{\prime}\right) \pi \in \mathcal{A}^{0}\right\}=\rho(x)-m
$$

for any $x \in \mathcal{X}$ and $m \in \mathbb{R}$.

The relation (1.16) confers to the risk acceptance family an additional economical meaning. In the theory of monetary risk measures, $\mathcal{A}_{0}$ is understood as the set of acceptable positions from a regulating agency's point of view. This regulating agency enforces then financial institutions possessing assets $x$ in the risk class $\mathcal{A}^{m}$ to reserve a liquid amount $m$ on a risk free bank account $\pi$ to ensure that $x+m \pi$ is acceptable in the sense that it belongs to $\mathcal{A}^{0}$.

It is well-known that any cash additive risk measure is convex, see [Delbaen, 2003, Frittelli and Rosazza Gianin, 2002, Cerreia-Vioglio et al., 2010] and the references therein. In [Cheridito and Kupper, 2009b] it is shown that any risk measure $\rho$ satisfying $\rho(m)=-m$ for all $m \in \mathbb{R}$ is convex exactly when it is cash additive.

Proposition 1.24. A cash additive risk measure $\rho$ is automatically convex.

To be self-contained, we present here a simple proof which relies on the special shape of the acceptance family of cash additive risk measures.

Proof. Given a cash additive risk measure $\rho$, Proposition 1.23 implies that the related risk acceptance family $\mathcal{A}$ fulfills the relation (1.16). Hence, for any $m, m^{\prime} \in \mathbb{R}$ and $\lambda \in] 0,1[$ follows

$$
\begin{aligned}
\lambda \mathcal{A}^{m}+(1-\lambda) \mathcal{A}^{m^{\prime}} & =\lambda \mathcal{A}^{0}-\lambda m \pi+(1-\lambda) \mathcal{A}^{0}-(1-\lambda) m^{\prime} \pi \\
& =\mathcal{A}^{0}-\left(\lambda m+(1-\lambda) m^{\prime}\right) \pi \\
& =\mathcal{A}^{\lambda m+(1-\lambda) m^{\prime}} .
\end{aligned}
$$

And so, by Proposition 1.16, $\rho$ is convex.

Example 1.25. We consider in the following examples that $\mathcal{X}=\mathbb{L}^{\infty}$ and denote by $\mathcal{M}_{1}(P)$ the set of probability measures $Q$ which are absolutely continuous with respect to $P$.

The celebrated mean variance risk measure introduced by MARKOwITz [1952],

$$
\rho(X):=-E[X]+\frac{\lambda}{2} \operatorname{Var}(X), \quad X \in \mathbb{L}^{\infty},
$$

is a cash additive risk measure which is monotone with respect to the trivial preorder but not with respect to the preorder "greater than $P$-almost surely". A monotone version with respect to the preorder "greater than $P$-almost surely" has been studied in [Maccheroni et al., 2009].

The average value at risk- $A V @ R$ for short-is defined as follows. Given the convex subset $\mathcal{Q}_{q} \subset \mathcal{M}_{1}(P)$ of those probability measures $Q$ whose densities $d Q / d P$ are 


\section{Risk Orders}

bounded from above by $1 / q$ for $q \in] 0,1[$

$$
A V @ R_{q}(X):=\sup _{Q \in \mathcal{Q}_{q}} E_{Q}[-X], \quad X \in \mathbb{L}^{\infty} .
$$

Another prominent example is the entropic risk measure given by

$$
\rho(X):=\ln (E[\exp (-X)]), \quad X \in \mathbb{L}^{\infty},
$$

which is a cash additive risk measure.

A last important class of cash additive risk measures suitable for optimization problems, is the optimized certainty equivalent introduced by BEN-TAL AND TEBoulle [1986] and defined as

$$
\rho(X):=-\sup _{m \in \mathbb{R}}\{m+E[u(X-m)]\}, \quad X \in \mathbb{L}^{\infty},
$$

where $u: \mathbb{R} \rightarrow[-\infty,+\infty[$ is an upper semicontinuous concave nondecreasing function such that $u(0)=0$ and $1 \in \partial u(0)$. An exhaustive study of the optimized certainty equivalent as a cash additive risk measure can be found in [Ben-Tal and Teboulle, 2007].

Recently, El Karoui and RavanelLi [2009] pointed out that in the framework of monetary risk measures, the risk free bank account $\pi$ could also be subject to interest rate uncertainty. In consequence, a higher amount of liquidity should be reserved today on the bank account $\pi$ to ensure that risky positions remain acceptable. For this purpose, they introduced the notion of cash subadditivity for convex risk measures, which has been extended to quasiconvex risk measures in [Cerreia-Vioglio et al., 2010], since cash subadditive risk measure are not automatically convex. Cash subadditive risk measures also appear naturally as the generators describing the one-step actualisation of dynamic cash additive risk measures for stochastic processes, [see Cheridito and Kupper, 2009a, Acciaio et al., 2010].

Definition 1.26. Any risk measure $\rho$ on $\mathcal{X}$ is cash subadditive if

$$
\rho(x+m \pi) \geq \rho(x)-m,
$$

for any $m>0$.

Remark 1.2\%. Another version of cash subadditivity is proposed in [Cerreia-Vioglio et al., 2010] where they require $\rho(x-m \pi) \leq \rho(x)-m$ for $m>0$. We here work with the interpretation and definition of cash subadditivity given by [El Karoui and Ravanelli, 2009].

Here again, it is possible to characterize cash subadditive risk measures by the properties of their related risk acceptance families. 
Proposition 1.28. Let $\rho$ be a cash subadditive risk measure and $\mathcal{A}$ its risk acceptance family. Then

$$
\mathcal{A}^{n} \subset \mathcal{A}^{n+m}+m \pi, \quad \text { for all } m>0 \text { and } n \in \mathbb{R} \text {. }
$$

Conversely, if a risk acceptance family $\mathcal{A}$ fulfills relation 1.21, the corresponding risk measure $\rho_{\mathcal{A}}$ is cash subadditive.

Proof. Let $\rho$ be a cash subadditive risk measure with corresponding risk acceptance family $\mathcal{A}$. For any $m>0, n \in \mathbb{R}$, and $x+m \pi \in \mathcal{A}^{n}$ follows

$$
n \geq \rho(x+m \pi) \geq \rho(x)-m,
$$

showing that $x \in \mathcal{A}^{m+n}$. Hence, $\mathcal{A}^{n}-m \pi \subset \mathcal{A}^{m+n}$.

Conversely, consider some risk acceptance family $\mathcal{A}$ fulfilling the relation (1.21) and with corresponding risk measure $\rho$. Theorem 1.10 yields

$$
\rho(x+m \pi)=\inf \left\{n \in \mathbb{R} \mid x+m \pi \in \mathcal{A}^{n}\right\} \geq \inf \left\{n \in \mathbb{R} \mid x \in \mathcal{A}^{n+m}\right\}=\rho(x)-m,
$$

for any $m>0$, showing that $\rho$ is a cash subadditive risk measure.

We finally illustrate with two examples how monetary risk measures - not necessarily cash additive nor cash subadditive - can be defined by economically motivated risk acceptance families.

Example 1.29 (Numéraire uncertainty). Notably for global acting financial institutions, it is reasonable for the regulating agency to require that risky positions have to be acceptable with respect to a basket of currencies—€, $£, ¥$ and $\$$ for instance - in reason of the different interest rate policies. The financial institutions face here some numéraire uncertainty to assess the risk ${ }^{9}$. Modelling this problem is particularly easy from the risk acceptance family point of view. Indeed, let $\mathcal{A}^{0}$ be the acceptance set given by the regulating institution and let $\mathcal{N} \subset \mathcal{K}$ be a set of possible numéraires. Define

$$
\mathcal{A}^{m}:=\left\{X \mid X+m \pi \in \mathcal{A}^{0} \text { for all } \pi \in \mathcal{N}\right\}=\bigcap_{\pi \in \mathcal{N}}\left\{\mathcal{A}^{0}-m \pi\right\}, \quad m \in \mathbb{R} .
$$

It is clear that $\mathcal{A}$ is a risk acceptance family and therefore defines a risk measure.

Example 1.30 (Liquidity costs). In contrast to the previous example, liquidity costs are in term of assessment of risk a short term problematic. Usually, financial firms are required to assess on a daily basis there financial exposition to risk. If this assessment suddenly increases due to some bubble burst or extraordinary event, as for example in the weeks following the default of Lehman Brothers by the 15th of September 2008 where unseen daily value at risk of banking institutions were observed, it is then confronted to the problem of unfolding some illiquid positions to provide the necessary liquidity to keep its overall position acceptable. This does not happen without liquidity costs,

\footnotetext{
${ }^{9}$ Note that the question of the interest rate uncertainty is similar, since the regulator requires acceptability then with respect to a set of possible interest rates.
} 


\section{Risk Orders}

which can even be overwhelming if the timing is short and the liquidity is scarce. As in [Cerreia-Vioglio et al., 2010] where this example was first introduced, we model this by modifying the translation invariance structure of cash additive risk acceptance family to reflect the risk induced by these additional costs, that is:

$$
\mathcal{A}^{0}=\mathcal{A}^{m}+(m-c(m)) \pi, \quad m \in \mathbb{R},
$$

whereby $c: \mathbb{R} \rightarrow \mathbb{R}$ is an increasing cost function with $c(0)=0$. Here, the amount of money needed to pull the risk of a position in $\mathcal{A}^{m}$ in the acceptable set $\mathcal{A}^{0}$ is, unlike cash additive risk acceptance family, greater than $m$. A robust representation is given in Section 3.1, Example 3.9. 


\section{Robust Representation of Risk Orders}

\subsection{A General Robust Representation Result}

The goal of this section is to provide a dual representation of risk orders. To this end, we however need some topological structure and therefore, from this point forward, we assume that $\mathcal{X}$ is a locally convex topological vector space $^{1}$. By $\mathcal{X}^{*}$, we denote its topological dual space endowed with the weak topology $\sigma\left(\mathcal{X}^{*}, \mathcal{X}\right)$. Unless explicitly precised, elements of the dual space $\mathcal{X}^{*}$ will be denoted by $x^{*}, y^{*}, \ldots$

We further assume that the preorder $\unrhd$ is upper semicontinuous, that is, the cone $\mathcal{K}=$ $\{x \in \mathcal{X} \mid x \geqslant 0\}$ is $\sigma\left(\mathcal{X}, \mathcal{X}^{*}\right)$-closed. The bipolar theorem, see for instance [Aliprantis and Border, 2006, Theorem 5.103], states that $x \unrhd y$ exactly when $\left\langle x^{*}, x-y\right\rangle \geq 0$ for all $x^{*}$ in the polar cone

$$
\mathcal{K}^{\circ}:=\left\{x^{*} \in \mathcal{X}^{*} \mid\left\langle x^{*}, x\right\rangle \geq 0 \quad \text { for all } x \in \mathcal{K}\right\}
$$

which is $\sigma\left(\mathcal{X}^{*}, \mathcal{X}\right)$-closed. Further, we denote by $\tilde{\mathcal{K}}$ those elements $\pi \unrhd 0$ which are strictly positive with respect to $\mathcal{K}^{\circ}$, that is

$$
\tilde{\mathcal{K}}=\left\{\pi \in \mathcal{K} \mid\left\langle x^{*}, \pi\right\rangle>0 \text { for any } x^{*} \in \mathcal{K}^{\circ} \backslash\{0\}\right\} .
$$

The preorder $\unrhd$ is called regular if there exists a strictly positive element, that is, $\tilde{\mathcal{K}} \neq \emptyset$. In that case, we can normalize the polar cone $\mathcal{K}^{\circ}$ in the sense that

$$
\mathcal{K}^{\circ}=\mathbb{R}_{+} \mathcal{K}_{\pi}^{\circ}=\left\{\lambda x^{*} \mid \lambda \geq 0 \text { and } x^{*} \in \mathcal{K}_{\pi}^{\circ}\right\}
$$

for any $\pi \in \tilde{\mathcal{K}}$, where

$$
\mathcal{K}_{\pi}^{\circ}=\left\{x^{*} \in \mathcal{K}^{\circ} \mid\left\langle x^{*}, \pi\right\rangle=1\right\},
$$

is called the normalized polar set with respect to $\pi$. Note that the trivial relation $\unrhd$ corresponds to the convex cone $\mathcal{K}=\{0\}$ which is is clearly not regular as $\tilde{K}=\emptyset$.

To illustrate the nature of these new elements, we briefly expose to what they concretely correspond in two of the settings presented in Section 1.1.

- Random variables: The set of $P$-almost surely bounded random variables $\mathcal{X}=$

\footnotetext{
${ }^{1}$ The study of $\mathcal{X}$ as a convex subset of a topological vector space space is postponed to Section 2.4.
} 
$\mathbb{L}^{\infty}$ admits the cone $\mathcal{K}=\mathbb{L}_{+}^{\infty}$ for the preorder "greater than $P$-almost surely". Depending on the considered topology we alternatively have

1. For the $\infty$-norm, the dual space $\mathcal{X}^{*}=b a(P)$ is the set of bounded finitely additive signed measures on $\mathscr{F}$ absolutely continuous with respect to $P$. The polar cone is the set of finitely additive measures denoted by $\mathcal{K}^{\circ}=b a_{+}(P)$. The preorder is regular. As mentioned previously, the vector $\pi \in \tilde{\mathcal{K}}$ is usually oriented in finance by the choice of a numéraire. We assume here that $\pi=$ 1 for which the normalized polar set $\mathcal{K}_{1}^{\circ}=\mathcal{M}_{1, f}(P)$ is the set of finitely additive probability measures $Q$ absolutely continuous with respect to $P$.

2. For the weak*-topology $\sigma\left(\mathbb{L}^{\infty}, \mathbb{L}^{1}\right)$, the dual space is $\mathcal{X}^{*}=\mathbb{L}^{1}$. In this case, $\mathcal{K}^{\circ}=\mathbb{L}_{+}^{1}$. Here again, the preorder is regular and, by means of the Radon-Nikodým theorem, $\mathcal{K}_{1}^{\circ}=\mathcal{M}_{1}(P)$ is the set of probability measures in $\mathcal{M}_{1, f}(P)$.

- Probability distributions: We consider on $c a_{c}$ and henceforth on $\mathcal{M}_{1, c}$ the weak*-topology $\sigma\left(c a_{c}, C\right)$ where $C:=C(I)$ is the set of continuous functions $u: I \rightarrow \mathbb{R}$. The dual pairing is given by $\langle u, \mu\rangle=\int u d \mu$. The stochastic orders presented in Section 1.1 were in fact already defined from their polar cones. With superscript referring to the respective order,

$$
\mathcal{K}^{i}=\left\{\mu \in c a_{c} \mid \int u d \mu \geq 0 \text { for any } u \in \mathcal{K}^{i, \circ}\right\}, \quad i=1,2,
$$

where

$$
\begin{aligned}
& \mathcal{K}^{1, \circ}=\{u \in C \mid u \text { is nondecreasing }\}, \\
& \mathcal{K}^{2, \circ}=\{u \in C \mid u \text { is nondecreasing and concave }\} .
\end{aligned}
$$

Notice that these orders are not regular since $\tilde{\mathcal{K}}^{1}=\tilde{\mathcal{K}}^{2}=\emptyset$.

Definition 2.1 (Lower Semicontinuous Risk Orders). A risk order $\succcurlyeq$ is said to be lower semicontinuous if $\mathcal{L}(x)=\{y \in \mathcal{X} \mid x \succcurlyeq y\}$ is $\sigma\left(\mathcal{X}, \mathcal{X}^{*}\right)$-closed for any $x \in \mathcal{X}$.

Under the assumption of lower semicontinuity, the existence of a lower semicontinuous numerical representation is far from being trivial. Under the stronger assumption of continuity, that is, both $\mathcal{L}(x)$ and $\mathcal{U}(x)$ are closed, Debreu [1954] in Euclidean spaces and further PELEG [1970] in general topological spaces proved such a result. A proof for the lower semicontinuous case was primarily addressed by RADER [1963] in Euclidean spaces and then corrected ${ }^{2}$ and generalized by Bosi AND MeHTA [2002] to topological spaces.

Proposition 2.2. A risk order $\succcurlyeq$ is separable and lower semicontinuous if and only if there exists a corresponding lower semicontinuous risk measure $\rho$. Moreover, the class of

23osi And Mehta [2002] pointed out that RADER [1963]'s proof was incorrect. 
corresponding lower semicontinuous risk measures is stable under lower semicontinuous increasing transformations.

Proof. The first part of the proposition is a direct application of the results in [Bosi and Mehta, 2002]. For the second part, let us define $\hat{\rho}=h \circ \rho$ for a lower semicontinuous risk measure $\rho: \mathcal{X} \rightarrow[-\infty, \infty]$ and a lower semicontinuous increasing function $h: \operatorname{Im}(\rho) \rightarrow$ $\mathbb{R}$. By relation (2.13),

$$
\{x \in \mathcal{X} \mid \hat{\rho}(x)=h \circ \rho(x) \leq m\}=\left\{x \in \mathcal{X} \mid \rho(x) \leq h^{(-1, r)}(m)\right\},
$$

and so $\hat{\rho}$ is a lower semicontinuous risk measure.

In fact, there exist numerical representations of lower semicontinuous risk orders which are not lower semicontinuous by taking an ad hoc non lower semicontinuous increasing transformation of a lower semicontinuous risk measure. However, the second part of the proposition states the stability of the class of lower semicontinuous numerical representations under lower semicontinuous increasing transformation. It can then be seen as a global characteristic - like quasiconvexity or monotonicity — but in a topological sense.

Remark 2.3. The risk acceptance family $\mathcal{A}_{\rho}$ corresponding to a lower semicontinuous risk measure $\rho$ is closed, that is, $\mathcal{A}_{\rho}^{m}$ is closed for any $m \in \mathbb{R}$. Conversely, the risk measure $\rho_{\mathcal{A}}$ corresponding to a closed risk acceptance family $\mathcal{A}$ is lower semicontinuous.

Aside the numerous technicalities, the core idea of the proof leading to the robust representation of the subsequent Theorem 2.7 is insightfull since the risk acceptance family plays a central role. To get an intuition of the objects in play and how they get involved, we informally sketch the key steps of the proof in the special case of random variables. To begin with, by way of relation (1.7), we express the risk measure $\rho$ in terms of its risk acceptance family

$$
\rho(X)=\inf _{m}\{\rho(X) \leq m\}=\inf _{m}\left\{X \in \mathcal{A}^{m}\right\} .
$$

We now exploit the fact that for each of these risk acceptance set $\mathcal{A}^{m}$ a cash additive risk measure $\rho^{m}$ can be defined through

$$
\rho^{m}(X)=\sup _{Q}\left\{E_{Q}[-X]-\alpha_{\min }(Q, m)\right\},
$$

where $\alpha_{\min }(Q, m)=\sup _{X \in \mathcal{A}^{m}} E_{Q}[-X]$ is the so called minimal penalty function ${ }^{3}$. In particular, $X \in \mathcal{A}^{m}$ exactly when $\rho^{m}(X) \leq 0$, so that

$$
\rho(X)=\inf _{m}\left\{\rho^{m}(X) \leq 0\right\}=\inf _{m}\left\{m \in \mathbb{R} \mid E_{Q}[-X] \leq \alpha_{\min }(Q, m) \text { for all } Q\right\} .
$$

\footnotetext{
${ }^{3}$ The term minimal penalty function was introduced in the theory of monetary risk measures; it is the smallest penalty function describing convex monetary risk measure in their robust representation, see [Föllmer and Schied, 2002].
} 


\section{Robust Representation of Risk Orders}

Without duality gap in interchanging the supremum over $Q$ with the infimum over $m$, we finally derive the robust representation

$$
\begin{aligned}
\rho(X) & =\sup _{Q} \inf _{m}\left\{m \in \mathbb{R} \mid E_{Q}[-X] \leq \alpha_{\min }(Q, m)\right\} \\
& =\sup _{Q} R\left(Q, E_{Q}[-X]\right)
\end{aligned}
$$

whereby $R$ is the left inverse of the nondecreasing function ${ }^{4} m \mapsto \alpha(Q, m)$, that is

$$
R(Q, s)=\inf \left\{m \in \mathbb{R} \mid s \leq \alpha_{\min }(Q, m)\right\} .
$$

Following this idea, we define the minimal penalty function of a risk acceptance family $\mathcal{A}$ by

$$
\alpha_{\text {min }}\left(x^{*}, m\right)=\sup _{x \in \mathcal{A}^{m}}\left\langle x^{*},-x\right\rangle, \quad x^{*} \in \mathcal{K}^{\circ} \text { and } m \in \mathbb{R} .
$$

Remark 2.4. Even if the risk acceptance family is right-continuous, the penalty function $m \mapsto \alpha_{\min }\left(x^{*}, m\right)$ is in general neither right nor left-continuous. Indeed, consider $\Omega=[0,1], \mathscr{F}=\mathscr{B}_{[0,1]}$ the Borel $\sigma$-algebra, and $P=d x$ the Lebesgue measure, and define $\mathcal{A}^{m}=\emptyset$ for $m<0, \mathcal{A}^{m}=\left\{X \in \mathbb{L}^{\infty} \mid X 1_{[m, 1]} \geq 0\right\}$ for $0 \leq m \leq 1$ and $\mathcal{A}^{m}=\mathbb{L}^{\infty}$ for $m>1$. Obviously, $\mathcal{A}$ is a closed risk acceptance family, and for $x^{*}=P$ holds $\alpha_{\min }\left(x^{*}, m\right)=-\infty$ for $m<0, \alpha_{\min }\left(x^{*}, m\right)=0$ for $m=0$ and $\alpha_{\min }\left(x^{*}, m\right)=+\infty$ for $m>0$ which is neither right nor left-continuous.

It is the left inverse of the minimal penalty function which will be the cornerstone of the robust representation and belongs to the following class of risk functions.

Definition 2.5. A risk function is a mapping $R: \mathcal{K}^{\circ} \times \mathbb{R} \rightarrow[-\infty,+\infty]$ which is nondecreasing and left-continuous in the second argument. The class of those risk functions is denoted by $\mathcal{R}$.

Further, if $\unrhd$ is regular, a normalized risk function with respect to $\pi \in \tilde{\mathcal{K}}$ is a mapping $R: \mathcal{K}_{\pi}^{\circ} \times \mathbb{R} \rightarrow[-\infty,+\infty]$ which is nondecreasing and left-continuous in the second argument. The class of those normalized risk functions with respect to $\pi$ is denoted by $\mathcal{R}_{\pi}$.

Note that in the case of regularity, the restriction of any risk function to $\mathcal{K}_{\pi}^{\circ} \times \mathbb{R}$ is a normalized risk function. As for the uniqueness of the representation, specific risk functions are in play, namely the maximal risk functions.

Definition 2.6. A risk function $R \in \mathcal{R}$ is said to be maximal if it has the following additional properties

(i) $R$ is jointly quasiconcave,

(ii) $R\left(\lambda x^{*}, s\right)=R\left(x^{*}, s / \lambda\right)$ for any $x^{*} \in \mathcal{K}^{\circ}, s \in \mathbb{R}$ and $\lambda>0$,

${ }^{4}$ Since the monotony implies $\mathcal{A}^{m} \subset \mathcal{A}^{n}$ whenever $m \leq n$. 
(iii) $R$ has a uniform asymptotic minimum, that is,

$$
\lim _{s \rightarrow-\infty} R\left(x^{*}, s\right)=\lim _{s \rightarrow-\infty} R\left(y^{*}, s\right)
$$

for any $x^{*}, y^{*} \in \mathcal{K}^{\circ}$,

( $i v)$ its right-continuous version, $R^{+}\left(x^{*}, s\right):=\inf _{s^{\prime}>s} R\left(x^{*}, s\right)$, is upper semicontinuous in the first argument.

The set of maximal risk functions is denoted by $\mathcal{R}^{\max }$.

Similarly, a normalized risk function $R \in \mathcal{R}_{\pi}$ is maximal if $R$ is jointly quasiconcave, $R$ has a uniform asymptotic minimum on $\mathcal{K}_{\pi}^{\circ}$ and $R^{+}$is upper semicontinuous in the first argument. The set of maximal normalised risk functions is denoted by $\mathcal{R}_{\pi}^{\max }$.

After this preliminary definitions and notations, we present our robust representation results.

Theorem 2.7. Any lower semicontinuous risk measure $\rho: \mathcal{X} \rightarrow[-\infty,+\infty]$ of a separable lower semicontinuous risk order $\succcurlyeq$ has a robust representation of the form

$$
\rho(x)=\sup _{x^{*} \in \mathcal{K}^{\circ}} R\left(x^{*},\left\langle x^{*},-x\right\rangle\right), \quad x \in \mathcal{X}
$$

for a unique maximal risk function $R \in \mathcal{R}^{\max }$ which is the left inverse of the minimal penalty function $\alpha_{\min }$.

Conversely, for any risk function $R \in \mathcal{R}$, the function $\rho$ defined by (2.6) is a lower semicontinuous risk measure.

In the case where the preorder $\unrhd$ is regular, we can even obtain a finer robust representation.

Theorem 2.8. Let $\triangleq$ be a regular preorder. Any lower semicontinuous risk measure $\rho: \mathcal{X} \rightarrow[-\infty,+\infty]$ of a separable lower semicontinuous risk order $\succcurlyeq$ has a robust representation of the form

$$
\rho(x)=\sup _{x^{*} \in \mathcal{K}_{\pi}^{\circ}} R\left(x^{*},\left\langle x^{*},-x\right\rangle\right), \quad x \in \mathcal{X},
$$

for a unique maximal normalized risk function $R \in \mathcal{R}_{\pi}^{\max }$ which is the left inverse of the minimal penalty function $\alpha_{\min }$.

Conversely, for any $R \in \mathcal{R}_{\pi}$, the function $\rho$ defined by (2.7) is a lower semicontinuous risk measure.

The proof of these two Theorems 2.7 and 2.8 is the subject of the next section 2.2.

As mentioned in the introduction, the one-to-one relation between risk measures $\rho$ and their risk functions $R \in \mathcal{R}^{\max }$ is crucial for the dual classification of risk measures and the respective risk orders and makes comparative statics meaningful. To this aim, Cerreia-Vioglio, Maccheroni, Marinacci, and Montrucchio [2008B] introduced the notion of a complete duality, in the sense that there exists a one-to-one 


\section{Robust Representation of Risk Orders}

relation between functions and their respective dual functions within a specified primal and dual class. For instance, the Fenchel-Moreau theorem states a complete duality between proper lower semicontinuous convex functions $f$ and their proper lower semicontinuous convex conjugates $f^{*}$. They give complete duality results for the class of monotone evenly ${ }^{5}$ quasiconvex functions and for different subclasses of it including the upper semicontinuous monotone quasiconvex functions. In the spirit of those results, Theorem 2.8 states the complete duality result for the class of lower semicontinuous quasiconvex functions, which is not treated in [Cerreia-Vioglio et al., 2010]. In contrast to [Cerreia-Vioglio et al., 2008b], Theorem 2.7 holds for functions which are monotone with respect to any preorder including the trivial one which corresponds to $\mathcal{K}=\{0\}$. Moreover, our setup is more general as we work in locally convex topological vector spaces rather than in $M$-spaces with an order unit. An $M$-space $E$ is a Riesz space equipped with a lattice norm $\|\cdot\|$ such that for any $x, y \in E_{+}$holds $\|x \vee y\|=\max \|x\|,\|y\|$, see [Aliprantis and Border, 2006]. Note for instance that the $\mathbb{L}^{p}$-spaces for $1 \leq p<\infty$ and $\left(\mathbb{L}^{\infty}, \sigma\left(\mathbb{L}^{\infty}, \mathbb{L}^{1}\right)\right)$ are not $M$-spaces.

The proofs of Theorems 2.7 and 2.8 are based on monetary risk measure theory and differ from the approach in the respective proofs in [Cerreia-Vioglio et al., 2008b]. The representation part of our proofs are in line with Penot And Volle [1990A, Proposition 3.6 and Theorem 3.8]. However, in [Penot and Volle, 1990a], the robust representation is stated in terms of elements in $\mathcal{X}^{*}$ rather than $\mathcal{K}^{\circ}$ or $\mathcal{K}_{\pi}^{\circ}$, and more important, uniqueness considerations and characterizations of the maximal risk function are not treated. For further references on quasiconvex duality theory, we refer to de Finetti [1949], Greenberg And Pierskalla [1973], Crouzeix [1980] and the references therein.

The denomination "maximal" risk function is justified by the following chracterisation result.

Proposition 2.9. Let $\rho: \mathcal{X} \rightarrow[-\infty,+\infty]$ be a lower semicontinuous risk measure which admits the following robust representations

$$
\rho(x)=\sup _{x^{*} \in \mathcal{K}^{\circ}} R\left(x^{*},\left\langle x^{*},-x\right\rangle\right)=\sup _{x^{*} \in \mathcal{K}^{\circ}} \tilde{R}\left(x^{*},\left\langle x^{*},-x\right\rangle\right), \quad x \in \mathcal{X},
$$

for some risk functions $R, \tilde{R}$ whereby $R \in \mathcal{R}^{\max }$. Then $R$ is point wise greater than $\tilde{R}$, that is

$$
R\left(x^{*}, s\right) \geq \tilde{R}\left(x^{*}, s\right), \quad \text { for all } x^{*} \in \mathcal{X}^{*} \text { and } s \in \mathbb{R} .
$$

Here again, the proof is postponed in the next section 2.2

Remark 2.10. On $M$-spaces, Cerreia-Vioglio et AL. [2008B] state a complete duality result between upper semicontinuous risk measures und risk functions which are jointly upper semicontinuous. The completeness in the lower semicontinuous case is more involved as the lower semicontinuity of the risk measure transfers into an upper semicontinuity condition for the right-continuous version of the risk function. As illus-

${ }^{5}$ The level sets are evenly convex, that is, they are the intersection of a family of open half-spaces. 
trated by the following examples, property $(i v)$ in Definition 2.6 cannot be expressed in terms of a semicontinuity condition for the risk function $R$.

Let $\mathcal{X}=\mathbb{R}^{2}, \mathcal{K}=\mathbb{R}_{+}^{2}, \pi=(1,1)$ in which case $\mathcal{K}_{\pi}^{\circ}=\{(p, 1-p) \mid p \in[0,1]\}$.

For the first example, we consider the risk function $R(p, s)=1_{\{s>p\}}$ which is obviously in $\mathcal{R}^{\max }$. However, $R(\cdot, 1 / 2)$ is not upper semicontinuous, as

$$
\{p \in[0,1] \mid R(p, 1 / 2) \geq 1 / 2\}=[0,1 / 2),
$$

is not closed.

For the second example, we show that maximal risk functions are in general not lower semicontinuous in the first argument. Indeed, within the setup of the previous example, we consider the maximal risk function $R(p, s)=1_{\{p \geq 1 / 2\}}$ for which $R=R^{+}$but $R(\cdot, s)$ is not lower semicontinuous for all $s \in \mathbb{R}$.

Remark 2.11. In Theorem 2.8 , the regularity assumption on the preorder $\unrhd$ cannot be dropped. For instance, let $\mathcal{X}=\mathbb{R}, \mathcal{K}=\{0\}$ so that $\mathcal{K}^{\circ}=\mathcal{X}^{*}=\mathbb{R}$ and consider the lower semicontinuous quasiconvex function $\rho(x):=x^{2}$, which is monotone with respect to the non-regular partial order $\mathcal{K}=\{0\}$. There exists no $\pi \in \mathbb{R} \backslash\{0\}$ such that

$$
\rho(x)=\sup _{x^{*} \in \mathcal{K}_{\pi}^{\circ}} R\left(x^{*},-x^{*} \cdot x\right), \quad \text { for all } x \in \mathbb{R},
$$

as $\mathcal{K}_{\pi}^{\circ}=\left\{x^{*} \in \mathbb{R} \mid x^{*} \pi=1\right\}$ reduces to the singleton $1 / \pi$ and $\rho(x)=x^{2}$ is different from any function $x \mapsto R(1 / \pi,-x / \pi)$ for some $R \in \mathcal{R}$, which by definition is either nondecreasing or nonincreasing depending on the sign of $\pi$.

\subsection{Proof of the General Robust Representation}

In this Section, we address the proof of the Theorems 2.7 and 2.8 as well as the proof of the Proposition 2.9, but beforehand, let us present a crucial concept for the sequel and the properties related to.

Definition 2.12 (Pseudo Inverse). A function $g: \mathbb{R} \rightarrow[-\infty,+\infty]$ is a pseudo inverse of a nondecreasing function $f: \mathbb{R} \rightarrow[-\infty,+\infty]$ if

$$
f^{-}(g(t)) \leq t \leq f^{+}(g(t)), \quad t \in \mathbb{R},
$$

with the convention that $f( \pm \infty)= \pm \infty$.

The left inverse $f^{(-1, l)}$ and the right inverse $f^{(-1, r)}$ are defined as

$$
\begin{array}{ll}
f^{(-1, l)}(t):=\sup \{s \in \mathbb{R} \mid f(s)<t\}=\inf \{s \in \mathbb{R} \mid f(s) \geq t\}, & t \in \mathbb{R}, \\
f^{(-1, r)}(t):=\sup \{s \in \mathbb{R} \mid f(s) \leq t\}=\inf \{s \in \mathbb{R} \mid f(s)>t\}, & t \in \mathbb{R} .
\end{array}
$$

The following proposition summarises known results on pseudo inverses, see also Penot and Volle [1990b], Mesiar et al. [2000], Föllmer and Schied [2004]. 


\section{Robust Representation of Risk Orders}

Proposition 2.13. Given a nondecreasing function $f: \mathbb{R} \rightarrow[-\infty,+\infty]$, any pseudo inverse $g$ of $f$ is nondecreasing, $f^{(-1, l)}=g^{-} \leq g \leq g^{+}=f^{(-1, r)}$ and all pseudo inverses of $f$ at most differ on a countable subset of $\mathbb{R}$. Furthermore, $f$ is itself a pseudo inverse of any of its pseudo inverses.

If $f$ is moreover left-continuous, then $g^{(-1, l)}=f$ for any pseudo inverse $g$ of $f$ and

$$
f(s) \leq t \quad \Longleftrightarrow \quad s \leq f^{(-1, r)}(t) .
$$

Symmetrically, if $f$ is right-continuous, then $g^{(-1, r)}=f$ for any pseudo inverse $g$ of $f$ and

$$
f(s) \geq t \quad \Longleftrightarrow \quad s \geq f^{(-1, l)}(t) .
$$

Finally, a nondecreasing function $f$ is respectively proper or convex if and only if any pseudo inverse of $f$ is respectively proper, or concave.

Proof. Consider a nondecreasing function $f: \mathbb{R} \rightarrow[-\infty,+\infty]$ and a pseudo inverse $g$ of $f$. By definition, $f^{(-1, l)} \leq g^{-} \leq g \leq g^{+} \leq f^{(-1, r)}$. Fix now a decreasing sequence $t_{n} \searrow t \in \mathbb{R}$. We have

$$
\{s \in \mathbb{R} \mid f(s)>t\}=\bigcup_{n \in \mathbb{N}}\left\{s \in \mathbb{R} \mid f(s)>t_{n}\right\},
$$

and therefore $f^{(-1 . r)}\left(t_{n}\right) \searrow f^{(-1, r)}(t)$ showing the right-continuity of $f^{(-1, r)}$. The fact that $g^{+}=f^{(-1, r)}$ is immediate as they only differ on the countable set of their respective discontinuities. As both are right-continuous, they thus coincide. A similar argumentation yields $f^{(-1, l)}$ is left-continuous and $f^{(-1, l)}=g^{-}$.

For any pseudo inverse $g$ of $f$ holds by definition $g(t) \geq s$ whenever $t>f(s)$ and therefore $g^{+}(f(s)) \geq s$. Conversely, $g(t) \leq s$ whenever $t<f(s)$ and thus $g^{-}(f(s)) \leq$ $s$, that is, $f$ a pseudo inverse of $g$. In particular, if $f$ is left continuous, respectively right continuous, then $g^{(-1, l)}=f$, respectively $g^{(-1, r)}=f$.

Further, the definition of $f^{(-1, l)}$ and $f^{(-1, r)}$ imply the implications " $\Rightarrow$ " of relations (2.13) and (2.14). Conversely, the relations $\left(f^{(-1, r)}\right)^{(-1, l)}=f$ if $f$ is left-continuous and $\left(f^{(-1, l)}\right)^{(-1, r)}=f$ if $f$ is right-continuous, show the reverse implications " $\Leftarrow$ ".

Finally, let $f$ be a lower semicontinuous proper concave function. From (2.13), it is clear that the epigraph of $f$ and the hypograph of $f^{(-1, r)}$ are related to each other by $(s, t) \in \operatorname{epi}(f)$ if and only if $(t, s) \in \operatorname{hypo}\left(f^{(-1, r)}\right)$ and therefore, follows the last assertion of the proposition.

Definition 2.14. By $\mathcal{P}^{\text {min }}$, we denote the set of minimal penalty functions, that is, those mappings $\alpha: \mathcal{K}^{\circ} \times \mathbb{R} \rightarrow[-\infty,+\infty]$ which are nondecreasing and left-continuous in the second argument and such that:

(a) $\alpha$ is convex in the first argument,

(b) $\alpha$ is positive homogeneous in the first argument,

(c) if there exists $x^{*} \in \mathcal{K}^{\circ}$ such that $\alpha\left(x^{*}, m\right)=-\infty$, then $\alpha(\cdot, m) \equiv-\infty$, 
(d) $\alpha$ is lower semicontinuous in the first argument.

Before we begin with the proof of Theorem 2.7, we need two lemmata the first of which states a one-to-one relation between $\mathcal{P}^{\min }$ and $\mathcal{R}^{\max }$.

Lemma 2.15. The left inverse of any function $\alpha \in \mathcal{P}^{\min }$ is in $\mathcal{R}^{\max }$, i.e.

$$
\alpha^{(-1, l)}\left(x^{*}, s\right):=\sup \left\{m \in \mathbb{R} \mid \alpha\left(x^{*}, m\right)<s\right\} \in \mathcal{R}^{\max } .
$$

The left inverse of any function $R \in \mathcal{R}^{\max }$ is in $\mathcal{P}^{\min }$, i.e.

$$
R^{(-1, l)}\left(x^{*}, m\right):=\sup \left\{s \in \mathbb{R} \mid R\left(x^{*}, s\right)<m\right\} \in \mathcal{P}^{\min } .
$$

Moreover, $\left(\alpha^{(-1, l)}\right)^{(-1, l)}=\alpha$, as well as $\left(R^{(-1, l)}\right)^{(-1, l)}=R$ for any $\alpha \in \mathcal{P}^{\text {min }}$ and $R \in \mathcal{R}^{\max }$.

Proof. Note that both minimal penalty functions and maximal risk functions are mappings from $\mathcal{K}^{\circ} \times \mathbb{R}$ to $[-\infty,+\infty]$, which are left-continuous and nondecreasing in the second argument. In the following, $\alpha$ is such a mapping from $\mathcal{K}^{\circ} \times \mathbb{R}$ to $[-\infty,+\infty]$. By Proposition 2.13, its left inverse denoted by $R$ is again a left-continuous nondecreasing function and in that case holds $\alpha=R^{(-1, l)}=\left(\alpha^{(-1, l)}\right)^{(-1, l)}, R=\alpha^{(-1, l)}=$ $\left(R^{(-1, l)}\right)^{(-1, l)}$ and $R^{+}=\alpha^{(-1, r)}$. Proposition 2.13 further implies that

$$
R^{+}\left(x^{*}, s\right) \geq m \quad \Longleftrightarrow \quad s \geq \alpha\left(x^{*}, m\right),
$$

for any $m, s \in \mathbb{R}$, and $x^{*} \in \mathcal{K}^{\circ}$.

We now show that $\alpha=R^{(-1, l)}$ is in $\mathcal{P}^{\text {min }}$ if and only if $R=\alpha^{(-1, l)}$ is in $\mathcal{R}^{\text {max }}$.

- Equivalence between condition $(a)$ for $\mathcal{P}^{\text {min }}$ and condition $(i)$ for $\mathcal{R}^{\text {max }}$. Firstly, the joint quasiconcavity of $R$ and the joint quasiconcavity of $R^{+}$are equivalent. Indeed, $R$ is the pointwise monotone limit of $\left(x^{*}, s\right) \mapsto R_{n}^{+}\left(x^{*}, s\right):=$ $R^{+}\left(x^{*}, s-1 / n\right)$ as well as $R^{+}$is the pointwise monotone limit of $\left(x^{*}, s\right) \mapsto$ $R_{n}\left(x^{*}, s\right):=R\left(x^{*}, s+1 / n\right)$ and

$$
\begin{aligned}
\left\{\left(x^{*}, s\right) \in \mathcal{K}^{\circ}\right. & \left.\times \mathbb{R} \mid R^{+}\left(x^{*}, s\right) \geq m\right\} \\
& =\bigcap_{n \in \mathbb{R}}\left\{\left(x^{*}, s\right) \in \mathcal{K}^{\circ} \times \mathbb{R} \mid R_{n}\left(x^{*}, s\right) \geq m\right\}, \\
\left\{\left(x^{*}, s\right) \in \mathcal{K}^{\circ}\right. & \left.\times \mathbb{R} \mid R\left(x^{*}, s\right) \geq m\right\} \\
& =\bigcap_{\varepsilon>0} \bigcup_{n \in \mathbb{R}}\left\{\left(x^{*}, s\right) \in \mathcal{K}^{\circ} \times \mathbb{R} \mid R_{n}^{+}\left(x^{*}, s\right)>m-\varepsilon\right\},
\end{aligned}
$$




\section{Robust Representation of Risk Orders}

both of which are convex for any $m \in \mathbb{R}$. Secondly, by use of relation (2.17) holds

$$
\begin{array}{r}
\left\{\left(x^{*}, s\right) \in \mathcal{K}^{\circ} \times \mathbb{R} \mid R^{+}\left(x^{*}, s\right) \geq m\right\}=\left\{\left(x^{*}, s\right) \in \mathcal{K}^{\circ} \times \mathbb{R} \mid s \geq \alpha\left(x^{*}, m\right)\right\} \\
=\operatorname{epi}(\alpha(\cdot, m)),
\end{array}
$$

for any $m \in \mathbb{R}$. Finally, a function is convex if and only its epigraph is convex.

- Equivalence between condition (b) for $\mathcal{P}^{\min }$ and condition $(i i)$ for $\mathcal{R}^{\max }$. If $\alpha$ is positive homogeneous in the first argument, then for any $\lambda>0$ we get

$$
\begin{aligned}
R\left(\lambda x^{*}, s\right)=\sup \{m \in \mathbb{R} \mid \alpha & \left.\left(\lambda x^{*}, m\right)<s\right\} \\
& =\sup \left\{m \in \mathbb{R} \mid \alpha\left(x^{*}, m\right)<s / \lambda\right\}=R\left(x^{*}, s / \lambda\right) .
\end{aligned}
$$

Conversely, under the assumption that $R\left(\lambda x^{*}, s\right)=R\left(x^{*}, s / \lambda\right)$ for all $\lambda>0$ it follows

$$
\begin{aligned}
\alpha\left(\lambda x^{*}, m\right)=\sup \{s \in \mathbb{R} \mid & \left.R\left(\lambda x^{*}, s\right)<m\right\} \\
& =\lambda \sup \left\{s / \lambda \in \mathbb{R} \mid R\left(x^{*}, s / \lambda\right)<m\right\}=\lambda \alpha\left(x^{*}, m\right) .
\end{aligned}
$$

- Equivalence between condition $(c)$ for $\mathcal{P}^{\text {min }}$ and condition (iii) for $\mathcal{R}^{\max }$. Define

$$
\begin{aligned}
& C:=\left\{\left(x^{*}, m\right) \in \mathcal{K}^{\circ} \times \mathbb{R} \mid \alpha\left(x^{*}, m\right)=-\infty\right\} \\
& D:=\left\{\left(x^{*}, m\right) \in \mathcal{K}^{\circ} \times \mathbb{R} \mid \lim _{s \rightarrow-\infty} R^{+}\left(x^{*}, s\right) \geq m\right\}
\end{aligned}
$$

It is clear that if $\alpha \in \mathcal{P}^{\text {min }}$, condition $(c)$ for $\mathcal{P}^{\text {min }}$ is equivalent to $C=\mathcal{K}^{\circ} \times J$ for either $\left.J=\emptyset, J=]-\infty, c_{0}\right]$ for $c_{0} \in \mathbb{R}$, or $J=\mathbb{R}$. On the other hand, condition (iii) for $\mathcal{R}^{\text {max }}$ holds if and only if $D=\mathcal{K}^{\circ} \times J$ for $J$ of the same type than before. Indeed, $D=\mathcal{K}^{\circ} \times \emptyset$ if and only if $\lim _{s \rightarrow-\infty} R^{+}\left(x^{*}, s\right)=-\infty$ for any $x^{*} \in \mathcal{K}^{\circ}$. Further, $D=\mathcal{K}^{\circ} \times \mathbb{R}$ if and only if, $R^{+} \equiv+\infty$. Last, $\left.\left.D=\mathcal{K}^{\circ} \times\right]-\infty, c_{0}\right]$ for $c_{0} \in \mathbb{R}$ if and only if $\lim _{s \rightarrow+\infty} R\left(x^{*}, s\right)=c_{0}$ for any $x^{*} \in \mathcal{K}^{\circ}$.

It remains to show that $C=D$. Indeed, relation (2.17) states that

$$
\begin{aligned}
C=\left\{\left(x^{*}, m\right)\right) \in \mathcal{K}^{\circ} \times \mathbb{R} \mid & \left.\alpha\left(x^{*}, m\right) \leq s \text { for all } s \in \mathbb{R}\right\} \\
& =\left\{\left(x^{*}, m\right) \mid m \leq R^{+}\left(x^{*}, s\right) \text { for all } s \in \mathbb{R}\right\}=D .
\end{aligned}
$$

- Equivalence between condition $(d)$ for $\mathcal{P}^{\min }$ and condition $(i v)$ for $\mathcal{R}^{\max }$. Using 
again relation $(2.17)$ yields

$$
\left\{x^{*} \in \mathcal{K}^{\circ} \mid R^{+}\left(x^{*}, s\right) \geq m\right\}=\left\{x^{*} \in \mathcal{K}^{\circ} \mid s \geq \alpha\left(x^{*}, m\right)\right\}
$$

for any $m, s \in \mathbb{R}$. This states the equivalence between the lower semicontinuity of $\alpha$ and the upper semicontinuity of $R^{+}$.

Let $\mathcal{P}_{0}^{\min }$ denote the set of positive homogeneous, lower semicontinuous and convex functions $\alpha: \mathcal{K}^{\circ} \rightarrow[-\infty,+\infty]$ such that if there exists $x^{*} \in \mathcal{K}^{\circ}$ with $\alpha\left(x^{*}\right)=-\infty$, then $\alpha \equiv-\infty$. In particular, $\alpha(\cdot, m) \in \mathcal{P}_{0}^{\min }$ for any $\alpha \in \mathcal{P}^{\min }$ and all $m \in \mathbb{R}$.

Lemma 2.16. Let $\mathcal{A} \subset \mathcal{X}$ be a $\sigma\left(\mathcal{X}, \mathcal{X}^{*}\right)$-closed, convex set such that $y \unrhd x$ with $x \in \mathcal{A}$ implies $y \in \mathcal{A}$. Then, there exists a unique $\alpha \in \mathcal{P}_{0}^{\min }$ such that

$$
x \in \mathcal{A} \Longleftrightarrow\left\langle x^{*},-x\right\rangle \leq \alpha\left(x^{*}\right) \quad \text { for all } x^{*} \in \mathcal{K}^{\circ}
$$

In this case, $\alpha$ is given as the support function of $-\mathcal{A}$, that is, the minimal ${ }^{6}$ penalty function

$$
\alpha\left(x^{*}\right)=\alpha_{\min }\left(x^{*}\right):=\sup _{x \in \mathcal{A}}\left\langle x^{*},-x\right\rangle, \quad x^{*} \in \mathcal{K}^{\circ} .
$$

If in addition $\mathcal{K}$ is regular then for any fixed $\pi \in \tilde{\mathcal{K}}$ one has

$$
x \in \mathcal{A} \Longleftrightarrow\left\langle x^{*},-x\right\rangle \leq \alpha\left(x^{*}\right) \quad \text { for all } x^{*} \in \mathcal{K}_{\pi}^{\circ}
$$

and $\alpha$ is unique in the set of all lower semicontinuous convex functions from $\mathcal{K}_{\pi}^{\circ}$ to $[-\infty,+\infty]$ such that if there exists $x^{*} \in \mathcal{K}_{\pi}^{\circ}$ with $\alpha\left(x^{*}\right)=-\infty$, then $\alpha \equiv-\infty$.

Proof. Let $\alpha_{\min }$ denote the support function of $-\mathcal{A}$ defined by relation (2.19). By definition, $\alpha_{\min } \in \mathcal{P}_{0}^{\min }$. We next show that $\alpha_{\min }$ fulfills relation $(2.18)$. The case $\mathcal{A}=\emptyset$ is obvious. If $\mathcal{A} \neq \emptyset$, the implication

$$
x \in \mathcal{A} \quad \Longrightarrow \quad\left\langle x^{*},-x\right\rangle \leq \sup _{y \in \mathcal{A}}\left\langle x^{*},-y\right\rangle=\alpha_{\min }\left(x^{*}\right), \quad \text { for all } x^{*} \in \mathcal{K}^{\circ} .
$$

is straightforward. Conversely, for any $x \in \mathcal{X} \backslash \mathcal{A}$, the separation theorem yields

$$
\left\langle x_{0}^{*},-x\right\rangle>\sup _{y \in \mathcal{A}}\left\langle x_{0}^{*},-y\right\rangle=\alpha_{\min }\left(x_{0}^{*}\right)
$$

for some $x_{0}^{*} \in \mathcal{X}^{*}$. However, the monotonicity of $\mathcal{A}$ implies that $\left\langle x_{0}^{*},-x\right\rangle>\left\langle x_{0}^{*},-y\right\rangle+$ $\left\langle x_{0}^{*},-k\right\rangle$ for some $y \in \mathcal{A}$ and all $k \in \mathcal{K}$. Hence, $0 \geq\left\langle x_{0}^{*},-k\right\rangle$ for all $k \in \mathcal{K}$, implying that $x_{0}^{*} \in \mathcal{K}^{\circ}$ and this shows the reverse implication in (2.18).

As for the uniqueness, suppose there exist $\alpha_{1}, \alpha_{2} \in \mathcal{P}_{0}^{\min }$ which represent $\mathcal{A}$ in the sense of (2.18). In case that $\alpha_{1}$ is identically $+\infty$ or $-\infty$, the same obviously holds for $\alpha_{2}$ and vice versa. From the condition $(c)$, it remains to show the case where both $\alpha_{1}$ and

\footnotetext{
${ }^{6}$ The minimality of the penalty function follows from the arguments given in [Föllmer and Schied, 2004, Theorem 4.15].
} 


\section{Robust Representation of Risk Orders}

$\alpha_{2}$ are proper. Define $\tilde{\alpha}_{i}=\alpha_{i}$ on $\mathcal{K}^{\circ}$ and $\tilde{\alpha}_{i}=+\infty$ on $\mathcal{K}^{\circ c}$ which remain proper, convex and lower semicontinuous. For the conjugates $\tilde{\alpha}_{i}^{*}(x)=\sup _{x^{*} \in \mathcal{K}^{\circ}}\left\{\left\langle x^{*}, x\right\rangle-\alpha_{i}\left(x^{*}\right)\right\}$ which are positive homogeneous follow $\tilde{\alpha}_{i}^{*}(x)=0$ if and only if $-x \in \mathcal{A}$. Thus, $\tilde{\alpha}_{1}^{*}=\tilde{\alpha}_{2}^{*}$ and the Fenchel-Moreau theorem yields $\tilde{\alpha}_{1}=\left(\tilde{\alpha}_{1}^{*}\right)^{*}=\left(\tilde{\alpha}_{2}^{*}\right)^{*}=\tilde{\alpha}_{2}$, that is, $\alpha_{1}=\alpha_{2}$ on $\mathcal{K}^{\circ}$.

Finally, in case that $\pi \in \tilde{\mathcal{K}} \neq \emptyset$, it follows that $\left\langle x^{*}, \pi\right\rangle>0$ for any $x^{*} \in \mathcal{K}^{\circ} \backslash\{0\}$ so that $x^{*} /\left\langle x^{*}, \pi\right\rangle \in \mathcal{K}_{\pi}^{\circ}$. Hence, $\mathcal{K}^{\circ}=\mathbb{R}_{+} \mathcal{K}_{\pi}^{\circ}$ and (2.18) is equivalent to

$$
x \in \mathcal{A} \Longleftrightarrow\left\langle\frac{x^{*}}{\left\langle x^{*}, \pi\right\rangle},-x\right\rangle \leq \alpha\left(\frac{x^{*}}{\left\langle x^{*}, \pi\right\rangle}\right) \text { for all } x^{*} \in \mathcal{K}^{\circ} \backslash\{0\} .
$$

Proof (Theorem 2.7.). Step 1. Let $\rho$ be a lower semicontinuous risk measure. Theorem 1.10 yields

$$
\rho(x)=\inf \left\{m \in \mathbb{R} \mid x \in \mathcal{A}^{m}\right\}, \quad x \in \mathcal{X} .
$$

Since any $\mathcal{A}^{m}$ is $\sigma\left(\mathcal{X}, \mathcal{X}^{*}\right)$-closed, convex and monotone, it follows from Lemma 2.16 that

$$
x \in \mathcal{A}^{m} \quad \Longleftrightarrow \quad\left\langle x^{*},-x\right\rangle-\alpha_{\min }\left(x^{*}, m\right) \leq 0 \quad \text { for all } x^{*} \in \mathcal{K}^{\circ},
$$

whereby $\alpha_{\min }(\cdot, m)$ is the support function of $\mathcal{A}^{m}$ as given by relation (2.19). Combining (2.23) and (2.24) yields

$$
\begin{aligned}
\rho(x) & =\inf \left\{m \in \mathbb{R} \mid\left\langle x^{*},-x\right\rangle \leq \alpha_{\min }\left(x^{*}, m\right) \text { for all } x^{*} \in \mathcal{K}^{\circ}\right\} \\
& =\inf \left\{m \in \mathbb{R} \mid\left\langle x^{*},-x\right\rangle \leq \alpha_{\min }^{-}\left(x^{*}, m\right) \text { for all } x^{*} \in \mathcal{K}^{\circ}\right\},
\end{aligned}
$$

for the left-continuous version $\alpha_{\min }^{-}$of $\alpha_{\min }$. The goal is to show that

$$
\rho(x)=\sup _{x^{*} \in \mathcal{K}^{\circ}} \inf _{m \in \mathbb{R}}\left\{m \mid\left\langle x^{*},-x\right\rangle \leq \alpha_{\min }^{-}\left(x^{*}, m\right)\right\} .
$$

To begin with, equation (2.25) implies:

$$
\rho(x) \geq \sup _{x^{*} \in \mathcal{K}^{\circ}} \inf _{m \in \mathbb{R}}\left\{m \mid\left\langle x^{*},-x\right\rangle \leq \alpha_{\min }^{-}\left(x^{*}, m\right)\right\} .
$$

As for the reverse inequality, suppose that $\rho(x)>-\infty$, otherwise (2.26) is trivial, and fix $m_{0}<\rho(x)$. Define $C=\left\{y \in \mathcal{X} \mid \rho(y) \leq m_{0}\right\}$, which is $\sigma\left(\mathcal{X}, \mathcal{X}^{*}\right)$-closed, convex, and such that $x \notin C$. By the hyperplane separation theorem, there exists a continuous linear functional $x_{0}^{*} \in \mathcal{X}^{*} \backslash\{0\}$ such that

$$
\left\langle x_{0}^{*}, x\right\rangle<\inf _{y \in C}\left\langle x_{0}^{*}, y\right\rangle
$$

By monotonicity of $\rho$ we have $C=C+\mathcal{K}$, hence (2.27) yields $\left\langle x_{0}^{*}, x\right\rangle<\left\langle x_{0}^{*}, y\right\rangle+\lambda\left\langle x_{0}^{*}, z\right\rangle$ 
for any $z \in \mathcal{K}, \lambda>0$ and $y \in C$. It follows that ${ }^{7} x_{0}^{*} \in \mathcal{K}^{\circ} \backslash\{0\}$. Thus

$$
\left\langle x_{0}^{*}, x\right\rangle<\inf _{y \in C}\left\langle x_{0}^{*}, y\right\rangle .
$$

Since $\mathcal{A}^{m} \subset C$ for all $m \leq m_{0},(2.28)$ yields

$$
\left\langle x_{0}^{*},-x\right\rangle-\alpha_{\min }\left(x_{0}^{*}, m\right) \geq\left\langle x_{0}^{*},-x\right\rangle-\sup _{y \in C}\left\langle x_{0}^{*},-y\right\rangle>0 .
$$

Hence

$$
\begin{aligned}
m_{0} \leq \sup _{x^{*} \in \mathcal{K}^{\circ}} \inf _{m \in \mathbb{R}}\left\{m \mid\left\langle x^{*},-x\right\rangle\right. & \left.\leq \alpha_{\min }\left(x^{*}, m\right)\right\} \\
& =\sup _{x^{*} \in \mathcal{K}^{\circ}} \inf _{m \in \mathbb{R}}\left\{m \mid\left\langle x^{*},-x\right\rangle \leq \alpha_{\min }^{-}\left(x^{*}, m\right)\right\} .
\end{aligned}
$$

Since the last relation holds for any $m_{0}<\rho(x)$ we derive

$$
\rho(x) \leq \sup _{x^{*} \in \mathcal{K}^{\circ}} \inf _{m \in \mathbb{R}}\left\{m \mid\left\langle x^{*},-x\right\rangle \leq \alpha_{\min }^{-}\left(x^{*}, m\right)\right\},
$$

and (2.26) is established.

Step 2. Since

$$
\alpha_{\min }^{-}\left(x^{*}, m\right)=\sup _{m^{\prime}<m} \sup _{x \in \mathcal{A}^{m^{\prime}}}\left\langle x^{*},-x\right\rangle=\sup _{x \in \mathcal{A}^{m-}}\left\langle x^{*},-x\right\rangle,
$$

where $\mathcal{A}^{m-}=\bigcup_{m^{\prime}<m} \mathcal{A}^{m^{\prime}}$, it is straightforward to check that $\alpha_{\text {min }}^{-} \in \mathcal{P}^{\text {min }}$. According to Lemma 2.15, the left inverse of $\alpha_{\min }^{-}$, denoted by $R$ is a maximal risk function, i.e., $R \in \mathcal{R}^{\max }$ and therefore relation (2.26) yields

$$
\rho(x)=\sup _{x^{*} \in \mathcal{K}^{\circ}} R\left(x^{*},\left\langle x^{*},-x\right\rangle\right), \quad x \in \mathcal{X} .
$$

As for the uniqueness, it is sufficient, according to Lemma 2.15, to show the uniqueness of $\alpha_{\min }^{-}$in $(2.26)$ as $\alpha_{\text {min }}^{-} \in \mathcal{P}^{\text {min }}$. Consider $\alpha_{1}, \alpha_{2} \in \mathcal{P}^{\text {min }}$ satisfying both

$$
\rho(x)=\sup _{x^{*} \in \mathcal{K}^{\circ}} \inf _{m \in \mathbb{R}}\left\{m \mid\left\langle x^{*},-x\right\rangle \leq \alpha_{i}\left(x^{*}, m\right)\right\}, \quad \text { for any } x \in \mathcal{X}, \quad i=1,2 .
$$

For any $m \in \mathbb{R}$ holds

$$
\begin{aligned}
\{x \mid \rho(x)<m\} & =\left\{x \mid \sup _{x^{*} \in \mathcal{K}^{\circ}} \inf _{n \in \mathbb{R}}\left\{n \mid\left\langle x^{*},-x\right\rangle \leq \alpha_{i}\left(x^{*}, n\right)\right\}<m\right\} \\
& =\left\{x \mid \text { there is } n<m \text { such that }\left\langle x^{*},-x\right\rangle \leq \alpha_{i}\left(x^{*}, n\right) \text { for all } x^{*} \in \mathcal{K}^{\circ}\right\} \\
& =\bigcup \bigcup_{n<m}\left\{x \mid\left\langle x^{*},-x\right\rangle \leq \alpha_{i}\left(x^{*}, n\right) \text { for all } x^{*} \in \mathcal{K}^{\circ}\right\} .
\end{aligned}
$$

\footnotetext{
${ }^{7}$ In case where $C=\emptyset, x_{0}^{*}$ can freely be chosen in $\mathcal{K}^{\circ} \backslash\{0\}$.
} 


\section{Robust Representation of Risk Orders}

The uniqueness result in Lemma 2.16 yields

$$
\alpha_{i}\left(x^{*}, m\right)=\sup _{x \in \mathcal{A}_{i}^{m}}\left\langle x^{*},-x\right\rangle
$$

for the $\sigma\left(\mathcal{X}, \mathcal{X}^{*}\right)$-closed convex sets $\mathcal{A}_{i}^{m}:=\left\{x \mid\left\langle x^{*},-x\right\rangle \leq \alpha_{i}\left(x^{*}, m\right)\right.$ for all $\left.x^{*} \in \mathcal{K}^{\circ}\right\}$. Thus, from relations $(2.32),(2.33)$ and the left-continuity of $\alpha_{i}\left(x^{*}, \cdot\right)$, follows

$$
\begin{aligned}
\alpha_{i}\left(x^{*}, m\right)=\sup _{n<m} \alpha_{i}\left(x^{*}, n\right)=\sup _{n<m} \sup _{x \in \mathcal{A}_{i}^{n}}\left\langle x^{*},-x\right\rangle \\
\left.=\bigcup_{n<m}^{\sup _{n}\left\langle\mathcal{A}^{m}\right.},-x\right\rangle=\sup _{\{x \mid \rho(x)<m\}}\left\langle x^{*},-x\right\rangle,
\end{aligned}
$$

and therefore $\alpha_{1}=\alpha_{2}$.

Step 3. Conversely, let $\rho(x):=\sup _{x^{*} \in \mathcal{K}^{\circ}} R\left(x^{*},\left\langle x^{*},-x\right\rangle\right)$ for some risk function $R \in \mathcal{R}$. Since $s \mapsto R\left(x^{*}, s\right)$ is nondecreasing, it follows that $\rho$ is monotone. Further, $s \mapsto$ $R\left(x^{*}, s\right)$ is left-continuous, quasiconvex and $x \mapsto\left\langle x^{*},-x\right\rangle$ is linear continuous for any $x^{*} \in \mathcal{K}^{\circ}$. Since by relation $(2.17)$ holds

$$
\left\{x \in \mathcal{X} \mid R\left(x^{*},\left\langle x^{*},-x\right\rangle\right) \leq m\right\}=\left\{x \in \mathcal{X} \mid\left\langle x^{*},-x\right\rangle \leq R^{(-1, r)}\left(x^{*}, m\right)\right\},
$$

it follows that $x \mapsto R\left(x^{*},-\left\langle x^{*}, x\right\rangle\right)$ is a lower semicontinuous quasiconvex function. This implies that the level sets

$$
\begin{aligned}
\{x \in \mathcal{X} \mid \rho(x) \leq m\} & =\left\{x \in \mathcal{X} \mid \sup _{x^{*} \in \mathcal{K}^{\circ}} R\left(x^{*},\left\langle x^{*},-x\right\rangle\right) \leq m\right\} \\
& =\bigcap_{x^{*} \in \mathcal{K}^{\circ}}\left\{x \in \mathcal{X} \mid R\left(x^{*},\left\langle x^{*},-x\right\rangle\right) \leq m\right\}
\end{aligned}
$$

are closed and convex for any $m \in \mathbb{R}$ and so $\rho$ is a lower semicontinuous risk measure.

Proof (of Theorem 2.8). Let $\rho$ be a lower semicontinuous risk measure. By Theorem 2.7 there exists a unique $R \in \mathcal{R}^{\max }$ whose restriction to $\mathcal{K}_{\pi}^{\circ} \times \mathbb{R}$ is in $\mathcal{R}_{\pi}^{\max }$ and such that

$$
\rho(x)=\sup _{x^{*} \in \mathcal{K}_{\pi}^{\circ}, \lambda>0} R\left(\lambda x^{*},\left\langle\lambda x^{*},-x\right\rangle\right)=\sup _{x^{*} \in \mathcal{K}_{\pi}^{\circ}} R\left(x^{*},\left\langle x^{*},-x\right\rangle\right) .
$$

The uniqueness follows as in the proof of Theorem 2.7. The arguments given in the proof of Lemma 2.15 imply that ${ }^{8} \mathcal{R}_{\pi}^{\max }$ and $\mathcal{P}_{\pi}^{\min }$ are in a one-to-one relation.

Proof (Proposition 2.9). Let $R, \tilde{R}$ be two risk functions such that

$$
\rho(x)=\sup _{x^{*} \in \mathcal{K}^{\circ}} \tilde{R}\left(x^{*},\left\langle x^{*},-x\right\rangle\right)=\sup _{x^{*} \in \mathcal{K}^{\circ}} R\left(x^{*},\left\langle x^{*},-x\right\rangle\right)
$$

whereby $R \in \mathcal{R}^{\max }$. By Theorem 2.7, $R$ is the left inverse of $\alpha_{\min }$.

${ }^{8} \mathcal{P}_{\pi}^{\min }$ denotes the set of those functions in $\mathcal{P}^{\text {min }}$ restricted to $\mathcal{K}_{\pi}^{\circ} \times \mathbb{R}$. 
Further, $\tilde{R}\left(x^{*},\left\langle x^{*},-x\right\rangle\right) \leq m$ for any $x^{*} \in \mathcal{K}^{\circ}, m \in \mathbb{R}$ and $x \in \mathcal{A}^{m}$. Relation (2.13) yields $\left\langle x^{*},-x\right\rangle \leq \tilde{R}^{(-1, r)}\left(x^{*}, m\right)$ for any $m \in \mathbb{R}$. Due to Lemma 2.16, $\alpha_{\text {min }}$ is the smallest function having this property, i.e., $\alpha_{\min }\left(x^{*}, m\right) \leq \tilde{R}^{(-1, r)}\left(x^{*}, m\right)$ for any $x^{*} \in \mathcal{X}^{*}$ and $m \in \mathbb{R}$. By Theorem 2.7, $R$ being the left inverse in the second argument of $\alpha_{\text {min }}$, it follows that $\tilde{R}\left(x^{*}, s\right) \leq R\left(x^{*}, s\right)$ for any $x^{*} \in \mathcal{X}^{*}$ and $s \in \mathbb{R}$.

\subsection{Special Cases}

We study here the consequences on the robust representation of additional properties of the risk measures discussed in Section 1.2. Similar results have been established in [Cerreia-Vioglio et al., 2008b] in the context of $M$-spaces.

Proposition 2.17. A risk measure $\rho$ with representation

$$
\rho(x)=\sup _{x^{*} \in \mathcal{K}^{\circ}} R\left(x^{*},\left\langle x^{*},-x\right\rangle\right), \quad x \in \mathcal{X},
$$

for some $R \in \mathcal{R}^{\max }$ is

(i) convex if and only if $s \mapsto R\left(x^{*}, s\right)$ is convex for any $x^{*} \in \mathcal{K}^{\circ}$.

(ii) positive homogeneous if and only if $R\left(x^{*}, \lambda s\right)=\lambda R\left(x^{*}, s\right)$ for any $x^{*} \in \mathcal{K}^{\circ}, \lambda>0$ and $s \in \mathbb{R}$.

(iii) scaling invariant if and only if $R\left(x^{*}, \lambda s\right)=R\left(x^{*}, s\right)$ for any $x^{*} \in \mathcal{K}^{\circ}, \lambda>0$ and $s \in \mathbb{R}$.

If the preorder is regular, a risk measure $\rho$ with representation

$$
\rho(x)=\sup _{x^{*} \in \mathcal{K}_{\pi}^{\circ}} R\left(x^{*},\left\langle x^{*},-x\right\rangle\right), \quad x \in \mathcal{X},
$$

for some $\pi \in \tilde{\mathcal{K}}$ and some $R \in \mathcal{R}_{\pi}^{\max }$ is

(iv) cash additive with respect to $\pi$ if and only if $R\left(x^{*}, s+m\right)=R\left(x^{*}, s\right)+m$ for any $x^{*} \in \mathcal{K}_{\pi}^{\circ}$ and $s, m \in \mathbb{R}$. In this case, $R\left(x^{*}, s\right)=s-\alpha_{\min }\left(x^{*}, 0\right)$.

(v) cash subadditive with respect to $\pi$ if and only if $R\left(x^{*}, s-m\right) \geq R\left(x^{*}, s\right)-m$ for any $x^{*} \in \mathcal{K}_{\pi}^{\circ}, s \in \mathbb{R}$ and $m>0$.

Proof. The proof is built on the respective properties of the acceptance family, which have been established in Propositions 1.16, 1.23, and 1.28.

( $i$ ) Convexity of $R$ in the second argument implies that $\rho$ is convex as the supremum of convex functions is convex. Conversely, suppose that $\rho$ is convex. By Proposition 
1.16 , for any $\left.m, m^{\prime} \in \mathbb{R}, \lambda \in\right] 0,1\left[\right.$ and $x^{*} \in \mathcal{K}^{\circ}$, it follows

$$
\begin{aligned}
& \alpha_{\min }\left(x^{*}, \lambda m+(1-\lambda) m^{\prime}\right)=\sup _{x \in \mathcal{A}^{\lambda m+(1-\lambda) m^{\prime}}}\left\langle x^{*},-x\right\rangle \\
& \geq \sup _{x \in \lambda \mathcal{A}^{m}+(1-\lambda) \mathcal{A}^{m^{\prime}}}\left\langle x^{*},-x\right\rangle=\lambda \sup _{x \in \mathcal{A}^{m}}\left\langle x^{*},-x\right\rangle+(1-\lambda) \sup _{x \in \mathcal{A}^{m^{\prime}}}\left\langle x^{*},-x\right\rangle \\
& =\lambda \alpha_{\min }\left(x^{*}, m\right)+(1-\lambda) \alpha_{\min }\left(x^{*}, m^{\prime}\right) .
\end{aligned}
$$

Hence, $m \mapsto \alpha_{\min }\left(x^{*}, m\right)$ is concave. But, since $R\left(x^{*}, \cdot\right)$ is the left inverse of $\alpha_{\min }\left(x^{*}, \cdot\right)$, by Proposition 2.13, it is therefore convex in the second argument.

(ii) Here also, $R$ positive homogeneous in the second component implies that $\rho$ is positive homogeneous. By Proposition 1.16, a similar computation as before yields

$$
\alpha_{\min }\left(x^{*}, \lambda m\right)=\lambda \alpha_{\min }\left(x^{*}, m\right), \quad x^{*}, m \in \mathcal{K}^{\circ} \times \mathbb{R}
$$

from which the reverse assertion follows.

(iii) Analogous to the proof of (ii).

(iv) The sufficiency is immediate. Conversely, suppose that $\rho$ is cash additive. Proposition 1.23 implies $\alpha_{\min }\left(x^{*}, m\right)=\alpha_{\min }\left(x^{*}, 0\right)+m$ from which we deduce $R\left(x^{*}, s\right)=$ $s-\alpha_{\min }\left(x^{*}, 0\right)$, and therefore $R\left(x^{*}, s+m\right)=R\left(x^{*}, s\right)+m$ for any $m, s \in \mathbb{R}$ and $x^{*} \in \mathcal{K}_{\pi}^{\circ}$.

$(v)$ The sufficiency is immediate. Conversely, if $\rho$ is cash subadditive, it follows from Proposition 1.23 that $\alpha_{\min }\left(x^{*}, n+m\right) \geq \alpha_{\min }\left(x^{*}, n\right)+m$ for any $n \in \mathbb{R}$ and $m>0$. Hence, follows

$$
\begin{aligned}
R\left(x^{*}, s-m\right) & =\sup _{n \in \mathbb{R}}\left\{n \mid \alpha_{\min }\left(x^{*}, n\right)<s-m\right\} \\
& \geq \sup _{n \in \mathbb{R}}\left\{n \mid \alpha_{\min }\left(x^{*}, n+m\right)<s\right\}=R\left(x^{*}, s\right)-m .
\end{aligned}
$$

for any $m>0$.

\subsection{Robust Representation of Risk Orders on Convex Sets}

In the Theorems 2.7 and 2.8, we assumed that the risky positions are in a vector space. This is not always the case, as for instance in the settings of probability distributions or consumption streams. Here, the idea is to extend the risk measure to a vector space and then apply Theorem 2.7. The main difficulty is yet to preserve the lower semicontinuity of the risk measure in the course of the extension. 
Throughout this subsection we assume that $\mathcal{X}$ is a convex subset of a vector space $\mathcal{V}$ and that the preorder $\unrhd$ corresponds to the convex cone $\mathcal{K} \subset \mathcal{V}$. By means of the risk acceptance family it is straightforward that any risk measure defined on $\mathcal{X}$ extends to $\mathcal{V}$

Proposition 2.18. Given a risk measure $\rho: \mathcal{X} \rightarrow[-\infty,+\infty]$ there is unique maximal risk measure $\hat{\rho}: \mathcal{V} \rightarrow[-\infty,+\infty]$ such that $\hat{\rho}$ restricted to $\mathcal{X}$ coincides with $\rho$.

Furthermore, the risk acceptance family $\hat{\mathcal{A}}$ of $\hat{\rho}$ is given by

$$
\hat{\mathcal{A}}^{m}=\bigcap_{n>m}\left(\mathcal{A}^{n}+\mathcal{K}\right), \quad m \in \mathbb{R}
$$

whereby, $\mathcal{A}$ is the risk acceptance family of $\rho$.

Proof. In the vector space $\mathcal{V}$ we define the family

$$
\hat{\mathcal{A}}^{m}:=\bigcap_{n>m}\left(\mathcal{A}^{n}+\mathcal{K}\right), \quad m \in \mathbb{R} .
$$

Immediate verification shows that $\hat{\mathcal{A}}$ is a risk acceptance family in $\mathcal{V}$. According to Theorem 1.10 it is enough to show that $\hat{\mathcal{A}}$ restricted to $\mathcal{X}$ coincides with $\mathcal{A}$, which follows from

$$
\hat{\mathcal{A}}^{m} \cap \mathcal{X}=\bigcap_{n>m}\left(\left(\mathcal{A}^{n}+\mathcal{K}\right) \cap \mathcal{X}\right)=\bigcap_{n>m} \mathcal{A}^{n}=\mathcal{A}^{m}
$$

since each $\mathcal{A}^{n}$ is monotone in $\mathcal{X}$. Finally, remark that $\hat{\mathcal{A}}$ is by construction the smallest risk acceptance family on $\mathcal{V}$ coinciding with $\mathcal{A}$ on $\mathcal{X}$, and therefore, the risk measure $\hat{\rho}$ corresponding to $\hat{\mathcal{A}}$ is the unique maximal risk measure coinciding with $\rho$ on $\mathcal{X}$.

In some circumstances, this extension might preserve the lower semicontinuity. This is the case if $\mathcal{X}$ is either closed or open in $\mathcal{V}$ as stated in the following theorem.

Theorem 2.19. Suppose that $\mathcal{X}$ is either closed or open. For any lower semicontinuous $^{9}$ risk measure $\rho: \mathcal{X} \rightarrow[-\infty,+\infty]$, there exists a unique maximal lower semicontinuous risk measure $\hat{\rho}: \mathcal{V} \rightarrow[-\infty,+\infty]$ which restricted to $\mathcal{X}$ coincides with $\rho$.

Furthermore, the risk acceptance family $\hat{\mathcal{A}}$ of $\hat{\rho}$ is given by

$$
\hat{\mathcal{A}}^{m}=\bigcap_{n>m} \overline{\mathcal{A}^{n}+\mathcal{K}}, \quad m \in \mathbb{R}
$$

whereby, $\mathcal{A}$ is the risk acceptance family of $\rho$.

Finally, the maximal risk function $\hat{R}$ in the robust representation of $\hat{\rho}$ is the left inverse of the minimal penalty function of the smaller acceptance family $\mathcal{A}$ given by

$$
\alpha_{\min }\left(x^{*}, m\right)=\sup _{x \in \mathcal{A}^{m}}\left\langle x^{*},-x\right\rangle,
$$

for any $x^{*} \in \mathcal{K}^{\circ}$ and $m \in \mathbb{R}$.

\footnotetext{
${ }^{9}$ The considered topology on $\mathcal{X}$ is the relative topology induced by the topology on $\mathcal{V}$.
} 


\section{Robust Representation of Risk Orders}

The proof of Theorem 2.19 is based on the following lemma

Lemma 2.20. Let $\mathcal{V}$ be a topological space and denote by $\bar{A}$ the closure of a set $A \subset \mathcal{V}$. The following two assertions hold

(i) $\bar{A} \cap B=\overline{A \cap B} \cap B$ for any sets $A, B \in \mathcal{V}$ where $B$ is closed.

(ii) $\bar{A} \cap B=\overline{A \cap B} \cap B$ for any sets $A, B \in \mathcal{V}$ where $B$ is open.

Proof. In both cases, the relation, " $\supset$ " is immediate. Let us show " $\subset$ " by assuming that $x \in \bar{A} \cap B$.

In case of $(i)$, it implies that $x \in G \cap B$ for any closed set $G \supset A$, and since $B$ is closed, it follows that $x \in G$ for any closed set $G \supset A \cap B$ and therefore $x \in \overline{A \cap B}$.

In case of (ii), by definition of the closure, for any neighborhood $V$ of $x$, holds $V \cap A \neq \emptyset$. Since $x \in B$ and $B$ is open and therefore a neighborhood of $x$, holds $V \cap(A \cap B)=(V \cap B) \cap A \neq \emptyset$. Thus, $x \in \overline{A \cap B}$, which shows the reverse inclusion.

Proof (of Theorem 2.19). Now, let $\rho: \mathcal{X} \rightarrow[-\infty,+\infty]$ be a lower semicontinuous risk measure, with respective risk acceptance family $\mathcal{A}$. Define the family $\hat{\mathcal{A}}$ by

$$
\hat{\mathcal{A}^{m}}=\bigcap_{n>m} \overline{\hat{\mathcal{A}}^{n}+\mathcal{K}}, \quad m \in \mathbb{R} .
$$

Immediate verification shows that $\hat{\mathcal{A}}$ is a risk acceptance family in $\mathcal{V}$. It is moreover closed by construction. According to Theorem 1.10, it is enough to show that $\hat{\mathcal{A}}$ restricted to $\mathcal{X}$ coincides with $\mathcal{A}$. From the lower semicontinuity of $\rho$ and the definition of the relative topology holds $\overline{\mathcal{A}^{m}} \cap \mathcal{X}=\mathcal{A}^{m}$. From the monotonicity of $\mathcal{A}$ holds $\left(\mathcal{A}^{m}+\mathcal{K}\right) \cap \mathcal{X}=\mathcal{A}^{m}$. Applying the Lemma 2.20 and using the right-continuity of $\mathcal{A}$ yields

$$
\begin{aligned}
\hat{\mathcal{A}}^{m} \cap \mathcal{X}=\bigcap_{n>m}\left(\overline{\mathcal{A}^{n}+\mathcal{K}} \cap \mathcal{X}\right)=\bigcap_{n>m}\left(\overline{\left(\mathcal{A}^{n}+\mathcal{K}\right) \cap \mathcal{X}} \cap \mathcal{X}\right) \\
\quad=\bigcap_{n>m}\left(\overline{\mathcal{A}^{n}} \cap \mathcal{X}\right)=\bigcap_{n>m} \mathcal{A}^{n}=\mathcal{A}^{m} .
\end{aligned}
$$

Finally, $\hat{\mathcal{A}}$ is by construction the smallest closed risk acceptance family on $\mathcal{V}$ coinciding with $\mathcal{A}$ on $\mathcal{X}$, and therefore, $\hat{\rho}$ given by $\hat{\mathcal{A}}$ is the unique maximal lower semicontinuous risk measure on $\mathcal{V}$ coinciding with $\rho$ on $\mathcal{X}$.

Finally, for any $n>m$ and any $x^{*} \in \mathcal{K}^{\circ}$ holds

$$
\begin{aligned}
\alpha_{\text {min }}^{+}\left(x^{*}, m\right) \leq \hat{\alpha}_{\text {min }}^{+}\left(x^{*}, m\right) & \leq \sup _{x \in \overline{\mathcal{A}^{n}+\mathcal{K}}}\left\langle x^{*},-x\right\rangle=\sup _{x \in \mathcal{A}^{n}+\mathcal{K}}\left\langle x^{*},-x\right\rangle \\
= & \sup _{x \in \mathcal{A}^{n}, y \in \mathcal{K}}\left\langle x^{*},-(x+y)\right\rangle=\sup _{x \in \mathcal{A}^{n}}\left\langle x^{*},-x\right\rangle=\alpha_{\min }\left(x^{*}, n\right),
\end{aligned}
$$

and therefore, $\alpha_{\min }^{+}=\hat{\alpha}_{\min }^{+}$which left inverse is $\hat{R}$. 


\subsection{Automatic Continuity Results}

It is sometimes possible to get the lower semicontinuity of the risk order automatically from the monotonicity. We will illustrate this fact in the cases of $\pi$-bounded risk orders and the von Neumann and Morgenstern representation.

\subsection{1. $\pi$-Bounded Preferences}

Throughout this subsection, $\mathcal{X}$ is a Fréchet space, that is, a complete metrizable vector space, and the cone $\mathcal{K}$ corresponding to the preorder $\triangleq$ is generating, that is, $\mathcal{X}=\mathcal{K}-\mathcal{K}$. In this context, it is well-known that any monotone convex function is automatically lower semicontinuous throughout the algebraic interior of its domain [see Borwein, 1987].

For a fixed $\pi \in \tilde{\mathcal{K}}$-implying that $\triangleq$ is regular - we say that a total preorder $\succcurlyeq$ on $\mathcal{X}$ is $\pi$-bounded if for any $x \in \mathcal{X}$

$$
m \pi \succcurlyeq x \succcurlyeq n \pi \text {. }
$$

for some $m, n \in \mathbb{R}$.

Remark 2.21. Given some $\pi \in \tilde{\mathcal{K}}$, a risk order $\succcurlyeq$ admitting some $\pi$-certainty equivalent ${ }^{10}$ is $\pi$-bounded and separable. In this case, if $m \pi \succ m^{\prime} \pi$ for any $m<m^{\prime}$, then there exists a corresponding risk measure $\rho$ such that $\rho(m \pi)=-m$ and $\mathcal{A}^{m}=\mathcal{L}(-m \pi)$ for any $m \in \mathbb{R}$.

Theorem 2.22. Suppose $\succcurlyeq$ is a $\pi$-bounded risk order on $\mathcal{X}$ such that

$$
\{\lambda \in[0,1] \mid x \succcurlyeq \lambda y+(1-\lambda) z\} \quad \text { is closed in }[0,1] \text {, }
$$

for any $x, y, z \in \mathcal{X}$ with $x \succcurlyeq y$. Then $\succcurlyeq$ is lower semicontinuous.

Proof. We have to show that $\mathcal{L}(x)$ is closed for all $x \in \mathcal{X}$. To this end, we assume that $\mathcal{L}(x)$ is different from $\emptyset$ and $\mathcal{X}$, as in those cases the closedness is obviously satisfied. The mapping

$$
\rho^{x}(y):=\inf _{n \in \mathbb{R}}\{n \mid y+n \pi \in \mathcal{L}(x)\}
$$

is cash additive and consequently a convex risk measure. By (2.37) and the monotonicity of $\mathcal{L}(x)$ the function $\rho^{x}$ is real valued. By [Borwein, 1987, Theorem 2.2], $\rho^{x}$ is a lower semicontinuous convex function and all its level sets are closed. Adapting the arguments of [Föllmer and Schied, 2004, Proposition 4.7] it follows

$$
\mathcal{L}(x)=\left\{y \in \mathcal{X} \mid \rho^{x}(y) \leq 0\right\}
$$

showing that $\mathcal{L}(x)$ is closed.

Remark 2.23. Any risk order on $\mathbb{L}^{\infty}$ which is monotone with respect to the preorder "greater than P-almost surely" is 1-bounded. Crucial though, is assumption (2.38) as

$\overline{{ }^{10} \text { For any } x \in \mathcal{X} \text { holds } x \sim m \pi \text { for some }} m \in \mathbb{R}$. 


\section{Robust Representation of Risk Orders}

illustrated by the following counter example. Consider the risk order on $\mathbb{R}$ induced by $\rho(x)=+\infty$ for $x \leq 0$ and $\rho(x)=-\infty$ for $x>0$. It is 1 -bounded but not lower semicontinuous as $\mathcal{L}(0)=] 0,+\infty[$.

\subsubsection{Affine Risk Measures on $\mathcal{M}_{1, c}$}

We give a representation result in the spirit of vON NEUMAnN and Morgenstern where the usual weak* continuity assumption is replaced by monotonicity with respect to the first or second stochastic order ${ }^{11}$.

Theorem 2.24. Let $\succcurlyeq$ be a risk order on $\mathcal{M}_{1, c}$ which is monotone with respect to either the first or the second stochastic order, which satisfies the Archimedian and the Independence axiom, such that for any $x \in I$ there is $y \in I$ with $\delta_{x} \succ \delta_{y}$ and for which

$$
\left\{\lambda \in[0,1] \mid \delta_{r} \succcurlyeq \lambda \delta_{s}+(1-\lambda) \delta_{t}\right\} \quad \text { is closed in }[0,1],
$$

for any $r, s, t \in I$ with $\delta_{r} \succcurlyeq \delta_{s}$. Then, the risk order $\succcurlyeq i s ~ \sigma\left(\mathcal{M}_{1, c}, C\right)$-lower semicontinuous and there exists a representing risk measure $\rho: \mathcal{M}_{1, c} \rightarrow \mathbb{R}$ with von Neumann and Morgenstern representation

$$
\rho(\mu)=\int u d \mu, \quad \mu \in \mathcal{M}_{1, c}
$$

whereby $u: \mathbb{R} \rightarrow \mathbb{R}$ is a nondecreasing right-continuous function.

The main difficulties here compared to the previous subsection is that $\mathcal{M}_{1, c}$ is not a vector space and that the $\sigma\left(c a_{c}, C\right)$-topology on $c a_{c}$ is not metrizable, and therefore $c a_{c}$ is not a Fréchet space for this topology, preventing us to directly apply the results in [Borwein, 1987].

Remark 2.25. In case of monotonicity with respect to the second stochastic order the function $u$ is continuous. On the other hand, the first stochastic order is not sufficient to guarantee the continuity of $u$. Indeed, consider the risk order corresponding to the risk measure $\rho(\mu)=-\int 1_{[0,+\infty} d \mu$, which $\sigma\left(c a_{c}, C\right)$-lower semincontinuous but not $\sigma\left(c a_{c}, C\right)$-continuous. Finally, as for the right-continuity of $u$ the condition (2.39) is necessary as it is easily seen from the risk order associated to the risk measure $\rho(\mu)=$ $-\int 1_{] 0,+\infty}\left[\mu\right.$ on $\mathcal{M}_{1, c}$.

Before we go onto the proof, let us first fix some notations. For $a, b \in \mathbb{R}$ with $[a, b] \subset I=] a_{0}, b_{0}\left[\right.$ where $-\infty \leq a_{0}<b_{0} \leq+\infty$ and $\nu \in \mathcal{M}_{1}$, we denote by $\mathcal{M}_{1}([a, b], \nu)$ the set of all $\mu \in \mathcal{M}_{1}$ which are absolutely continuous with respect to $\nu$ and such that $\mu([a, b])=1$. On $\mathcal{M}_{1}([a, b], \nu)$, we define the preorder $\mu_{1} \unrhd^{\nu} \mu_{2}$ if and only if $\int u d \mu_{1} \geq \int u d \mu_{2}$ for any continuous nondecreasing function $u:[a, b] \rightarrow \mathbb{R}$. On $\mathcal{M}_{1}([a, b], \nu)$, we consider the weak ${ }^{*}$-topology $\sigma\left(\mathbb{L}^{1}([a, b], \nu), \mathbb{L}^{\infty}([a, b], \nu)\right)$ where

\footnotetext{
${ }^{11}$ In between, this result has been improved in its proof and requires weaker assumptions. This is the subject of a paper by Delbaen et al. [2010].
} 
$\mathbb{L}^{p}([a, b], \nu):=\mathbb{L}^{p}([a, b], \mathscr{B}([a, b]), \nu)$ for $p=1, \infty$. Recall that the space $c a([a, b], \nu)$ of signed measures absolutely continuous with respect to $\nu$ and with support in $[a, b]$ corresponds to $\mathbb{L}^{1}([a, b], \nu)$. The preorder $\unrhd^{\nu}$ induces a preorder on $\mathbb{L}^{1}([a, b], \nu)$ by the cone

$$
\mathcal{K}(\nu)=\left\{h \in \mathbb{L}^{1}([a, b], \nu) \mid \int h u d \nu \geq 0 \text { for any continuous nondecreasing } u\right\} .
$$

Throughout, $\lambda^{l e b}$ denotes the Lebesgue measure on the Borel $\sigma$-algebra in $\mathbb{R}$.

The proof of Theorem 2.24 is based on the following technical lemmata.

Lemma 2.26. The polar cone of $\mathcal{K}^{\circ}(\nu)$ corresponding to $\unrhd^{\nu}$ is given by

$$
\mathcal{K}^{\circ}(\nu)=\{u:[a, b] \rightarrow \mathbb{R} \mid u=\bar{u} \nu \text {-a.s. for some nondecreasing function } \bar{u}: \mathbb{R} \rightarrow \mathbb{R}\} \text {. }
$$

Proof. Let us denote by $\mathcal{H}$ the right hand side of (2.40). First, $\mu_{1} \unrhd^{\nu} \mu_{2}$ if and only if $\int u d \mu_{1} \geq \int u d \mu_{2}$ for any $u \in \mathcal{H}$. Indeed, the necessary part is clear since any continuous nondecreasing function is in $\mathcal{H}$. Conversely, the Lebesgue theorem of dominated convergence implies that if $\mu_{1} \nabla^{\nu} \mu_{2}$, then $\int u d \mu_{1} \geq \int u d \mu_{2}$ for any $u \in \mathcal{H}$, since any nondecreasing function $u \in \mathcal{H}$ is a $\nu$-almost sure limit of a sequence of continuous increasing functions uniformly bounded by $|u(a)|+|u(b)|$.

Since $\mathcal{H}$ is a convex cone, according to the bipolar Theorem, see [Aliprantis and Border, 2006, Theorem 5.103], it is thus sufficient to show that $\mathcal{H}$ is a closed set in the $\sigma\left(\mathbb{L}^{\infty}([a, b], \nu), \mathbb{L}^{1}([a, b], \nu)\right)$-topology, from which would follow (2.40). Due to the Krein-Šmulian theorem, it is sufficient to show that for any $r>0$,

$$
\mathcal{H} \cap\left\{u \in \mathbb{L}^{\infty}([a, b], \nu) \mid\|u\|_{\infty} \leq r\right\}
$$

is $\sigma\left(\mathbb{L}^{\infty}([a, b], \nu), \mathbb{L}^{1}(\nu)\right)$-closed. According to [Föllmer and Schied, 2004, Lemma A.64], this is equivalent to the $\nu$-almost sure closure of

$$
\mathcal{H} \cap\left\{u \in \mathbb{L}^{\infty}([a, b], \nu) \mid\|u\|_{\infty} \leq r\right\}
$$

which is from the definition of $\mathcal{H}$ obviously the case.

Lemma 2.27. Let $\rho: \mathcal{M}_{1, c} \rightarrow \mathbb{R}$ be an affine risk measure which is monotone with respect to the first stochastic order and such that $x \mapsto \rho\left(\delta_{x}\right)$ is right-continuous and $\rho\left(\delta_{x}\right)>\inf _{y \in I} \rho\left(\delta_{y}\right)$ for all $x \in I$. Then, for any $m \in \mathbb{R}$ there $i s[\bar{a}, \bar{b}] \subset I$ such that for all $[a, b] \supset[\bar{a}, \bar{b}]$, all $\nu \in \mathcal{M}_{1}$ and any nondecreasing function $u \in \mathbb{L}^{\infty}([a, b]$, $\nu)$ there exists a sequence of nondecreasing continuous functions $u_{j} \in C([a, b])$ satisfying $u_{j} \rightarrow u$ $\nu$-almost surely and

$$
\limsup _{j \rightarrow \infty} \sup _{\mu \in \mathcal{A}^{m} \cap \mathcal{M}_{1}([a, b], \nu)}-\int u_{j} d \mu \leq \sup _{\mu \in \mathcal{A}^{m} \cap \mathcal{M}_{1}([a, b], \nu)}-\int u d \mu
$$




\section{Robust Representation of Risk Orders}

Proof. If $u$ is a càdlàg ${ }^{12}$ function, there exists a sequence $u_{j}$ of continuous nondecreasing functions converging from above $\nu$-almost surely to $u$. Since $u_{j} \geq u \nu$-almost surely, it follows

$$
\sup _{\mu \in \mathcal{A}^{m} \cap \mathcal{M}_{1}([a, b], \nu)}-\int u_{j} d \mu \leq \sup _{\mu \in \mathcal{A}^{m} \cap \mathcal{M}_{1}([a, b], \nu)}-\int u d \mu,
$$

for any $[a, b] \subset I$ and so holds (2.41). Thus, it is enough to show the lemma for a sequence of nondecreasing càdlàg functions converging $\nu$-almost surely to $u$.

If $m \leq \inf _{y \in I} \rho\left(\delta_{y}\right)$, then $\mathcal{A}^{m} \cap \mathcal{M}_{1}([a, b], \nu)=\emptyset$ for all $[a, b] \subset I$ in which case (2.41) is obviously satisfied. Otherwise, in case that $m>\inf _{y \in I} \rho\left(\delta_{y}\right)$ there exists $[\bar{a}, \bar{b}] \in I$ such that for any $[a, b] \supset[\bar{a}, \bar{b}]$, holds $\rho\left(\delta_{b}\right)<m$. Given $\varepsilon>0$, we have

$$
\left\|u-\left(v+\sum_{n=1}^{N} \beta_{n} 1_{] y_{l}, b\right]}\right)\right\|_{\infty} \leq \varepsilon .
$$

where $v$ is the càdlàg part of $u, a \leq y_{1}<\cdots<y_{N}<b$, and $\beta_{1}, \ldots, \beta_{N}>0$ for some $N \in \mathbb{N}$. The parameter $N$ as well as the reals $y_{1}, \ldots, y_{N}$ and $\beta_{1}, \ldots, \beta_{N}$ depends on $\varepsilon$ since these are the left continuous jumps of $u$ of size bigger than $\varepsilon$. In particular, for $\varepsilon_{1}>\varepsilon_{2}$, holds $\left\{y_{1}^{\varepsilon_{1}}, \ldots, y_{N_{\varepsilon_{1}}}^{\varepsilon_{1}}\right\} \subset\left\{y_{1}^{\varepsilon_{2}}, \ldots, y_{N_{\varepsilon_{2}}}^{\varepsilon_{2}}\right\}$.

It is then sufficient to show that there exists $\xi_{0}>0$ such that for any $0<\xi<\xi_{0}$ holds ${ }^{13}$

$$
\begin{aligned}
\sup _{\mu \in \mathcal{A}^{m} \cap \mathcal{M}_{1}([a, b], \nu)}-\int(v & \left.+\sum_{n=1}^{N} \beta_{n} 1_{\left[y_{n}+\xi, b\right]}\right) d \mu \\
\leq & \sup _{\mu \in \mathcal{A}^{m} \cap \mathcal{M}_{1}([a, b], \nu)}-\int\left(v+\sum_{n=1}^{N} \beta_{n} 1_{] y_{n}, b\right]}\right) d \mu+\varepsilon
\end{aligned}
$$

Indeed, relation (2.43) holds then for $\varepsilon_{j}=1 / j$ and a sequence $\xi_{j}>0$ converging to 0 , in which case, $u_{j}=v+\sum_{n_{j}=1}^{N_{j}} \beta_{n_{j}}^{j} 1_{\left[y_{n_{j}}^{j}+\xi_{j}, b\right]}$ is a càdlàg process converging $\nu$-almost surely to $u$ and fulfills

$$
\begin{aligned}
\sup _{\mu \in \mathcal{A}^{m} \cap \mathcal{M}_{1}([a, b], \nu)}-\int\left(v+\sum_{n_{j}=1}^{N_{j}} \beta_{n_{j}}^{j} 1_{\left[y_{n_{j}}^{j}+\xi_{j}, b\right]}\right) d \mu \leq \\
\leq \sup _{\mu \in \mathcal{A}^{m} \cap \mathcal{M}_{1}([a, b], \nu)}-\int\left(v+\sum_{n_{j}=1}^{N_{j}} \beta_{n_{j}}^{j} 1_{] y_{n_{j}}^{j}, b\right]}\right) d \mu+1 / j \\
\leq \sup _{\mu \in \mathcal{A}^{m} \cap \mathcal{M}_{1}([a, b], \nu)}-\int u d \mu+2 / j
\end{aligned}
$$

\footnotetext{
$12 \mathrm{~A}$ function is càdlàg if it is right-continuous with left limits.

${ }^{13}$ It is clear that if relation $(2.43)$ holds for one $\xi_{0}>0$, it holds for any $0<\xi<\xi_{0}$ since $v+$ $\sum_{n=1}^{N} \beta_{n} 1_{\left[y_{n}+\xi, b\right]} \geq v+\sum_{n=1}^{N} \beta_{n} 1_{\left[y_{n}+\xi_{0}, b\right]}$.
} 


\subsection{Automatic Continuity Results}

To show the relation (2.43), suppose by way of contradiction that there exists a sequence $0<\xi_{k}<\min _{j=1, \ldots, N-1}\left|y_{j+1}-y_{j}\right|$ converging to 0 and a sequence $\mu_{k} \in$ $\mathcal{A}^{m} \cap \mathcal{M}_{1}([a, b], \nu)$ such that

$$
-\int\left(v+\sum_{n=1}^{N} \beta_{n} 1_{\left[y_{n}+\xi_{k}, b\right]}\right) d \mu_{k}>\sup _{\mu \in \mathcal{A}^{m} \cap \mathcal{M}_{1}([a, b], \nu)}-\int\left(v+\sum_{n=1}^{N} \beta_{n} 1_{] y_{n}, b\right]}\right) d \mu+\varepsilon
$$

We further can suppose, by translation, that $v+\sum_{n=1}^{N} \beta_{n} 1_{\left[y_{n}+\xi_{k}, b\right]}$ is positive. We will need a bit more space for our argumentation in the sense that the sequence $\mu_{k}$ can be chosen in a strictly smaller risk level $\mathcal{A}^{\tilde{m}} \subset \mathcal{A}^{m}$ for $\tilde{m}<m$. Indeed, since $\rho\left(\delta_{b}\right)<m$, there exists $\lambda \in] 0,1\left[\right.$ and $\tilde{m}<m$ such that $\tilde{\mu}_{k}:=\lambda \mu_{k}+(1-\lambda) \delta_{b} \in \mathcal{A}^{\tilde{m}}$ and

$$
\begin{aligned}
-\int\left(v+\sum_{n=1}^{N} \beta_{n} 1_{\left[y_{n}+\xi_{k}, b\right]}\right) d \tilde{\mu}_{k} & \\
& >\sup _{\mu \in \mathcal{A}^{m} \cap \mathcal{M}_{1}([a, b], \nu)}-\int\left(v+\sum_{n=1}^{N} \beta_{n} 1_{] y_{n}, b\right]}\right) d \mu+\frac{\varepsilon}{2} .
\end{aligned}
$$

Pick $z_{n}^{k}$ in the interval $\left[y_{n}, y_{n}+\xi_{k}\right.$ [ such that $\tilde{\mu}_{k}(] z_{n}^{k}, y_{n}+\xi_{k}[) \leq 1 / k$, for $n=1, \ldots, N$ and define the probability distributions

$$
\bar{\mu}_{k}:=\left\{\begin{array}{ll}
\tilde{\mu}_{k} & \text { outside } \bigcup_{n=1}^{N}\left[y_{n}, z_{n}^{k}\right] \\
\tilde{\mu}_{k}\left(\left[y_{n}, z_{n}^{k}\right]\right) \delta_{z_{n}^{k}} & \text { on }\left[y_{n}, z_{n}^{k}\right] \text { for all } n=1, \ldots, N
\end{array} .\right.
$$

Since $\bar{\mu}_{k}$ dominates $\tilde{\mu}$ in the first stochastic order, $\bar{\mu}_{k} \in \mathcal{A}^{\tilde{m}} \cap \mathcal{M}_{1}([a, b], \nu)$. Similarly, we define

$$
\hat{\mu}_{k}:=\left\{\begin{array}{ll}
\tilde{\mu}_{k} & \text { outside } \bigcup_{n=1}^{N}\left[y_{n}, z_{n}^{k}\right] \\
\tilde{\mu}_{k}\left(\left[y_{n}, z_{n}^{k}\right]\right) \delta_{y_{n}} & \text { on }\left[y_{n}, z_{n}^{k}\right] \text { for all } n=1, \ldots, N
\end{array} .\right.
$$

which belongs to $\mathcal{M}_{1}([a, b], \nu)$ and for which clearly holds $\bar{\mu}(] z_{n}^{k}, \xi_{k}[) \leq 1 / k$. By definition of $\hat{\mu}_{k}$ and since $v$ is non decreasing, holds

$$
\begin{aligned}
& \left|-\int\left(v+\sum_{n=1}^{N} \beta_{n} 1_{\left[y_{n}+\xi_{k}, b\right]}\right) d \tilde{\mu}_{k}+\int\left(v+\sum_{n=1}^{N} \beta_{n} 1_{] y_{n}, b\right]}\right) d \hat{\mu}_{k}\right| \\
& \left.\left.\quad \leq \sum_{n=1}^{N}\left|\int_{\left[y_{n}, z_{n}^{k}\right]} v d\left(\tilde{\mu}_{k}-\hat{\mu}_{k}\right)\right|+\sum_{n=1}^{N} \beta_{n} \mid \tilde{\mu}_{k}\left(\left[y_{n}+\xi_{k}, b\right]\right)-\hat{\mu}_{k}(] y_{n}, b\right]\right) \mid \\
& \left.\left.=\sum_{n=1}^{N} \int_{\left[y_{n}, z_{n}^{k}\right]}\left(v-v\left(y_{n}\right)\right) d \tilde{\mu}_{k}+\sum_{n=1}^{N} \beta_{n} \mid \tilde{\mu}_{k}\left(\left[y_{n}+\xi_{k}, b\right]\right)-\hat{\mu}_{k}(] z_{n}^{k}, b\right]\right) \mid \\
& \leq \sum_{n=1}^{N}\left(v\left(z_{n}^{k}\right)-v\left(y_{n}\right)\right) \tilde{\mu}_{k}\left(\left[y_{n}, z_{n}^{k}\right]\right)+\sum_{n=1}^{N} \beta_{n} \tilde{\mu}_{k}(] z_{n}^{k}, y_{n}+\xi_{k}[)
\end{aligned}
$$




\section{Robust Representation of Risk Orders}

Both last sums tends to 0 as $k$ goes to infinity, since $v$ is càdlàg and $\tilde{\mu}_{k}(] z_{n}^{k}, y_{n}+\xi_{k}[)$ is assumed to be smaller than $1 / k$. By means of the affinity of $\rho$, denoting by $\breve{\mu}$ the measure equal to $\tilde{\mu} / \tilde{\mu}_{k}\left[\left(\cup_{n=1}^{N}\left[y_{n}, z_{n}^{k}\right]\right)^{c}\right]$ outside $\cup_{n=1}^{N}\left[y_{n}, z_{n}^{k}\right]$ and zero otherwise, holds

$$
\begin{aligned}
& \rho\left(\bar{\mu}_{k}\right)=\rho\left(\tilde{\mu}_{k}\left(\left(\cup_{n=1}^{N}\left[y_{n}, z_{n}^{k}\right]\right)^{c}\right) \breve{\mu}_{k}+\sum_{n=1}^{N} \tilde{\mu}_{k}\left(\left[y_{n}, z_{n}^{k}\right]\right) \delta_{z_{n}^{k}}\right) \\
& =\tilde{\mu}_{k}\left(\left(\cup_{n=1}^{N}\left[y_{n}, z_{n}^{k}\right]\right)^{c}\right) \rho\left(\breve{\mu}_{k}\right)+\sum_{n=1}^{N} \tilde{\mu}_{k}\left(\left[y_{n}, z_{n}^{k}\right]\right) \rho\left(\delta_{z_{n}^{k}}\right),
\end{aligned}
$$

and

$$
\begin{aligned}
& \rho\left(\hat{\mu}_{k}\right)=\rho\left(\tilde{\mu}_{k}\left(\left(\cup_{n=1}^{N}\left[y_{n}, z_{n}^{k}\right]\right)^{c}\right) \breve{\mu}_{k}+\sum_{n=1}^{N} \tilde{\mu}_{k}\left(\left[y_{n}, z_{n}^{k}\right]\right) \delta_{y_{n}}\right) \\
& =\tilde{\mu}_{k}\left(\left(\cup_{n=1}^{N}\left[y_{n}, z_{n}^{k}\right]\right)^{c}\right) \rho\left(\breve{\mu}_{k}\right)+\sum_{n=1}^{N} \tilde{\mu}_{k}\left(\left[y_{n}, z_{n}^{k}\right]\right) \rho\left(\delta_{y_{n}}\right),
\end{aligned}
$$

Together with the right-continuity of $x \mapsto \rho\left(\delta_{x}\right)$ it implies

$$
\rho\left(\hat{\mu}_{k}\right)-\rho\left(\bar{\mu}_{k}\right)=\sum_{n=1}^{N} \tilde{\mu}_{k}\left(\left[y_{n}, z_{n}^{k}\right]\right)\left(\rho\left(\delta_{y_{n}}\right)-\rho\left(\delta_{z_{n}^{k}}\right)\right) \underset{k \rightarrow+\infty}{\longrightarrow} 0 .
$$

Therefore, since $\bar{\mu}_{k} \in \mathcal{A}^{\tilde{m}} \cap \mathcal{M}_{1}([a, b], \nu)$, there exists some $k_{0} \in \mathbb{N}$ such that $\hat{\mu}_{k} \in$ $\mathcal{A}^{m} \cap \mathcal{M}_{1}([a, b], \nu)$ for all $k \geq k_{0}$. But then relation (2.46) yields

$$
\begin{array}{r}
\liminf _{k \rightarrow \infty}-\int\left(v+\sum_{n=1}^{N} \beta_{n} 1_{\left[y_{n}+\xi_{k}, b\right]}\right) d \tilde{\mu}_{k}=\liminf _{k \rightarrow \infty}-\int\left(v+\sum_{n=1}^{N} \beta_{n} 1_{] y_{n}, b\right]}\right) d \hat{\mu}_{k} \\
\leq \sup _{\mu \in \mathcal{A}^{m} \cap \mathcal{M}_{1}([a, b], \nu)}-\int\left(v+\sum_{n=1}^{N} \beta_{n} 1_{] y_{n}, b\right]}\right) d \mu,
\end{array}
$$

in contradiction to relation (2.45), ending the proof of the lemma.

Lemma 2.28. For $[a, b] \subset I, \nu \in \mathcal{M}_{1}$ with $\lambda^{l e b}+\delta_{b} \ll \nu$ and any nondecreasing continuous function $u \in C([a, b])$ holds

$$
\alpha_{\min }^{[a, b]}(u, m):=\sup _{\mu \in \mathcal{A}_{[a, b]}^{m}}-\int u d \mu=\sup _{\mu \in \mathcal{A}_{[a, b]}^{m}, \mu \ll \nu}-\int u d \mu
$$

where $\mathcal{A}_{[a, b]}^{m}:=\left\{\mu \in \mathcal{M}_{1}([a, b]) \mid \rho(\mu) \leq m\right\}$ and $m \in \mathbb{R}$.

Proof. For any $n \in \mathbb{N}$, we define a grid $a=x_{0}^{n}<x_{1}^{n}<\cdots<x_{N_{n}}^{n}=b$ satisfying $x_{j+1}^{n}-x_{j}^{n} \leq 1 / n$. For fixed $\mu \in \mathcal{A}_{[a, b]}^{m}$, let $\mu^{n}$ be a measure of the form $\mu^{n}=\alpha_{n} \delta_{b}+$ $\sum_{j=1}^{N_{n}-1} \lambda_{j, n}^{l e b}$ where $\alpha_{n}=\mu\left(\left[x_{N_{n}-1}^{n}, x_{N_{n}}^{n}\right]\right)$ and $\lambda_{j, n}^{l e b} \ll \lambda^{l e b}$ with support in $\left[x_{j}^{n}, x_{j+1}^{n}\right)$ such 


\subsection{Automatic Continuity Results}

that $\lambda_{j, n}^{l e b}\left(\left[x_{j}^{n}, x_{j+1}^{n}\right)\right)=\mu\left(\left[x_{j-1}^{n}, x_{j}^{n}\right)\right)$. By construction, follows that $\mu^{n} \ll \delta_{b}+\lambda^{l e b} \ll \nu$ and the monotonicity with respect to the first stochastic order yields $\mu^{n} \in \mathcal{A}_{[a, b]}^{m}$. Since $u$ is uniformly continuous on the compact interval $[a, b]$, we deduce

$$
-\int u d \mu=\lim _{n \rightarrow \infty}-\int u d \mu^{n}
$$

from which (2.47) follows.

Proposition 2.29. Any affine risk measure $\rho: \mathcal{M}_{1, c} \rightarrow \mathbb{R}$ which is monotone with respect to the first stochastic order is automatically continuous with respect to the variational norm $\|\cdot\|$.

Proof. Step 1. We first extend $\rho$ on a vector space. To this end, we pick a vector $\delta_{c_{0}}$ with $c_{0} \in I$ and consider the spanned vector space

$$
\mathcal{V}:=\operatorname{span}\left(\mathcal{M}_{1, c}-\delta_{c_{0}}\right)=\left\{\mu \in c a_{c} \mid \mu(I)=0\right\}
$$

Indeed, it is clear that the left hand side is included in the right hand side. Conversely, for $\mu \in c a_{c}$ with $\mu(I)=0$, write $\mu=\mu^{+}-\mu^{-}$for $\mu^{+}, \mu^{-} \in c a_{c+}$. In particular, $\mu^{+}(I)=\mu^{-}(I)$ implying that $\mu=\lambda\left(\bar{\mu}^{+}-\delta_{c_{0}}\right)-\lambda\left(\bar{\mu}^{-}-\delta_{c_{0}}\right)$ with $\bar{\mu}^{+}=\mu^{+} / \mu^{+}(I) \in$ $\mathcal{M}_{1, c}$, resp. $\bar{\mu}^{-}=\mu^{-} / \mu^{-}(I) \in \mathcal{M}_{1, c}$ and $\lambda=\mu^{+}(I)$, which states the reverse inclusion.

The risk measure $\hat{\rho}$ on $\mathcal{M}_{1, c}-\delta_{c_{0}}$ defined as

$$
\hat{\rho}(\mu):=\rho\left(\mu+\delta_{c_{0}}\right)-\rho\left(\delta_{c_{0}}\right)
$$

extends to a unique linear risk measure $\hat{\rho}$ on the vector space $\mathcal{V}$. Indeed, we first consider a Hamel basis $\left(\nu_{i}\right)_{i \in \mathcal{I}}$ of $\mathcal{V}$ consisting of elements ${ }^{14}$ of $\mathcal{M}_{1, c}-\delta_{c_{0}}$. Define $\hat{\rho}(\mu)=\sum_{k=1}^{n} \lambda_{k} \rho\left(\nu_{i_{k}}\right)$ for any $\mu \in \mathcal{V}$ with expression $\mu=\sum_{k=1}^{n} \lambda_{k} \nu_{i_{k}}$ for $i_{1}, \ldots, i_{n} \in \mathcal{I}$ which is well-defined.

We check now that $\rho=\hat{\rho}$ on $\mathcal{M}_{1, c}-\delta_{c_{0}}$. Due to Proposition 1.19, for any element of $\mathcal{M}_{1, c}-\delta_{c_{0}}$ of the form $\mu=\sum_{k=1}^{n} \lambda_{k} \nu_{k}$ with $\nu_{i} \in \mathcal{M}_{1, c}-\delta_{c_{0}}$ and $\sum_{k=1}^{n} \lambda_{k}=1$, holds $\rho(\mu)=\sum_{k=1}^{n} \lambda_{k} \rho\left(\nu_{k}\right)$. Thus, for any $\mu \in \mathcal{M}_{1, c}-\delta_{c_{0}}$ with expression $\mu=\sum_{k=1}^{n} \lambda_{k} \nu_{i_{k}}$

\footnotetext{
${ }^{14}$ This is not precisely the statement of the Theorem of Hamel, but a minor modification of its proof based on the axiom of choice does the job. Indeed, consider the class $\left(S_{\alpha}\right)_{\alpha \in \mathcal{J}}$ of all free basis in $\mathcal{M}_{1, c}-\delta_{c_{0}}$ partially ordered for the inclusion. In this class, take a chain $\left(S_{\alpha}\right)_{\alpha \in \mathcal{O}}$ and define $S_{\mathcal{O}}=\bigcup_{\alpha \in \mathcal{O}} S_{\alpha}$ which is still a subset of $\mathcal{M}_{1, c}-\delta_{c_{0}}$ and such that $S_{\mathcal{O}} \supset S_{\alpha}$ for all $\alpha \in \mathcal{O}$. It is also a free basis, as for any finite linear combination summing up to zero $\sum_{k=1}^{n} \lambda_{k} \nu_{\alpha_{k}}=0$, there exists some $\alpha \in \mathcal{O}$ such that all $\nu_{\alpha_{k}}$ are in $S_{\alpha}$, and as $S_{\alpha}$ is a basis, the coefficients $\lambda_{k}$ are all zero. Therefore, $S_{\mathcal{O}}$ is a true upper bound in $\left(S_{\alpha}\right)_{\alpha \in \mathcal{O}}$ of the chain $\left(S_{\alpha}\right)_{\alpha \in \mathcal{O}}$. Applying Zorn's lemma, there exists a maximal element $S$ in $\left(S_{\alpha}\right)_{\alpha \in \mathcal{J}}$. This maximal element generates $\mathcal{V}$ since otherwise there would exist some $\nu \in \mathcal{M}_{1, c}-\delta_{c_{0}}$ linearly independent of $S$ in contradiction to the maximality of $S$.
} 


\section{Robust Representation of Risk Orders}

holds

$$
\begin{aligned}
\rho(\mu)=\rho\left(\sum_{k=1}^{n} \lambda_{k} \nu_{i_{k}}\right) & =\rho\left(\sum_{k=1}^{n} \lambda_{k} \nu_{i_{k}}+\left(1-\sum_{k=1}^{n} \lambda_{k} \nu_{i_{k}}\right) 0\right) \\
= & \sum_{k=1}^{n} \lambda_{k} \rho\left(\nu_{i_{k}}\right)+\left(1-\sum_{k=1}^{n} \lambda_{k} \nu_{i_{k}}\right) \rho(0)=\sum_{k=1}^{n} \lambda_{k} \rho\left(\nu_{i_{k}}\right)=\hat{\rho}(\mu) .
\end{aligned}
$$

We are left to show that this extension remains monotone with respect to $\unrhd$. Since $\unrhd$ is a vector order and $\hat{\rho}$ is affine, it suffices to show that $\hat{\rho}(\mu) \leq 0$ for any $\mu \unrhd 0$. For such an element, write $\mu=\mu^{(1)}-\mu^{(2)}$ with $\mu^{(l)}=\sum_{k=1}^{n_{l}} \lambda_{l, k} \nu_{i_{l, k}}$ where all $\lambda_{l, k}$ are positive for $l=1,2$. Next choose $\alpha>0$ small enough ${ }^{15}$ such that $\alpha \mu+(1-\alpha) \mu^{(2)}=$ $\sum_{k=1}^{m} \beta_{k} \nu_{j_{k}}$ for which all $\beta_{k}$ are positive. Rescale then everything by $\gamma>0$ such that $\gamma\left[\alpha \mu+(1-\alpha) \mu^{(2)}\right]$ and $\gamma(1-\alpha) \mu^{(2)}$ are in $\mathcal{M}_{1, c}-\delta_{c_{0}}$, which is possible since $\lambda_{2, k}$ and $\beta_{k}$ are positive, the $\nu_{i}$ are elements of $\mathcal{M}_{1, c}-\delta_{c_{0}}$ and $0 \in \mathcal{M}_{1, c}-\delta_{c_{0}}$. Since $\triangleq$ is a vector order, we have $\gamma\left[\alpha \mu+(1-\alpha) \mu^{(2)}\right] \triangleq \gamma(1-\alpha) \mu^{(2)}$, and therefore, monotonicity of $\rho$ implies $\rho\left(\gamma\left[\alpha \mu+(1-\alpha) \mu^{(2)}\right]\right) \leq \rho\left(\gamma(1-\alpha) \mu^{(2)}\right)$. The risk measures $\rho$ and $\hat{\rho}$ coinciding on $\mathcal{M}_{1, c}-\delta_{c_{0}}$ and $\hat{\rho}$ being linear, we finally get that $\hat{\rho}(\mu) \leq 0$

The uniqueness is immediate since any two affine extensions have to coincide on the Hamel basis which is made of elements of $\mathcal{M}_{1, c}-\delta_{c_{0}}$.

Step 2. Any $\mu \in \mathcal{V}$ can be decomposed in $\mu=\mu^{+}-\mu^{-}$for some positive measures $\mu^{+}, \mu^{-} \in \mathcal{V} \cap \mathcal{K}^{1}$. Indeed, for $\mu \in \mathcal{V}$, we denote by $\left.\left.F_{\mu}(t):=\mu(] a_{0}, t\right]\right)$ the cumulative distribution function of $\mu$ which satisfies $F_{\mu}\left(a_{0}\right)=F_{\mu}\left(b_{0}\right)=0$. Since $t \mapsto F_{\mu}(t)$ has bounded variation, the same holds for $F_{\mu}^{+}:=\max \left(F_{\mu}, 0\right)$ and $F_{\mu}^{-}:=\max \left(-F_{\mu}, 0\right)$ and we can therefore define the measures $\mu^{-}:=-d F_{\mu}^{+}$and $\mu^{+}:=-d F_{\mu}^{-}$, which by construction satisfy

$$
\mu^{+}-\mu^{-}=d F_{\mu}^{+}-d F_{\mu}^{-}=d F_{\mu}=\mu
$$

and $\mu^{+}(I)=\mu^{-}(I)=0$ as $F_{\mu}^{ \pm}\left(a_{0}\right)=F_{\mu}^{ \pm}\left(b_{0}\right)=0$, showing that $\mu^{ \pm} \in \mathcal{V}$. Moreover, $\mu^{ \pm} \in \mathcal{K}^{1}$ which follows by partial integration,

$$
\int f d \mu^{ \pm}=-\int f d F_{\mu}^{ \pm}=\int F_{\mu}^{ \pm} d f \geq 0, \quad \text { for any } f \in \mathcal{K}^{1, \circ},
$$

as $d f$ is a nonnegative measure.

Step 3. The function $\hat{\rho}: \mathcal{V} \rightarrow \mathbb{R}$ is continuous with respect to the variational norm $\|\cdot\|$ on $c a_{c}$ and as a consequence $\mu \mapsto \rho(\mu)=\hat{\rho}\left(\mu-\delta_{0}\right)+\rho\left(\delta_{0}\right)$ for $\mu \in \mathcal{M}_{1, c}$ is also $\|\cdot\|-$ continuous. Indeed, by way of contradiction, there exists a sequence $\mu_{k} \in \mathcal{V}$ such that $\left\|\mu_{k}\right\|=1$ and $\hat{\rho}\left(\mu_{k}\right) \geq 2^{k}$, which decomposes in $\mu_{k}=\mu_{k}^{+}-\mu_{k}^{-}$, where $\mu_{k}^{+}, \mu_{k}^{-} \in \mathcal{V} \cap \mathcal{K}^{1}$, as introduced in the previous step. Since $\mathcal{V}$ is complete, it follows that $\mu:=\sum_{k \geq 1} 2^{-k} \mu_{k}^{+} \in$

${ }^{15}$ In fact for $0<\alpha<1 / 2$. 
$\mathcal{V}$. Since $\hat{\rho}\left(\mu_{k}^{+}\right) \geq 0$ for all $k \in \mathbb{N}$, we end up with

$$
\hat{\rho}(\mu) \geq \sum_{k=1}^{n} 2^{-k} \hat{\rho}\left(\mu_{k}^{+}\right) \geq \sum_{k=1}^{n} 2^{-k} \hat{\rho}\left(\mu_{k}\right)=n,
$$

in contradiction to the assumption that $\hat{\rho}(\mu) \in \mathbb{R}$.

We are now ready for the proof of Theorem 2.24 .

Proof. It is enough to prove the theorem for risk measures $\rho$ which are monotone with respect to the first stochastic order, as any risk measure which is monotone with respect to the second stochastic order is in particular monotone with respect to the first stochastic order.

Step 1. Due to Proposition 2.29, the risk measure $\mu \mapsto \rho(\mu)=\hat{\rho}\left(\mu-\delta_{0}\right)+\rho\left(\delta_{0}\right)$ for $\mu \in$ $\mathcal{M}_{1, c}$ is $\|\cdot\|$-continuous. We next show, for any interval $[a, b] \subset I$, big enough according to Lemma 2.27, that the restriction of $\rho$ to $\mathcal{M}_{1}([a, b])$ is $\sigma\left(\mathcal{M}_{1}([a, b]), C([a, b])\right)$-lower semicontinuous, or equivalently that

$$
\mathcal{A}_{[a, b]}^{m}=\left\{\mu \in \mathcal{M}_{1}([a, b]) \mid \rho(\mu) \leq m\right\}
$$

is closed for all $m \in \mathbb{R}$ in the weak*-topology. To do so, we will prove that

$$
\mu \in \mathcal{A}_{[a, b]}^{m} \quad \Longleftrightarrow \quad-\int u d \mu \leq \alpha_{\min }^{[a, b]}(u, m) \quad \text { for all nondecreasing } u \in C([a, b]),
$$

where $\alpha_{\min }^{[a, b]}(u, m)$ has been defined in $(2.47)$. The implication " $\Rightarrow$ " is obvious. In order to show " $\Leftarrow$ " we assume that $\hat{\mu} \notin \mathcal{A}_{[a, b]}^{m}$. Then, there exists $\nu \in \mathcal{M}_{1}$ with $\hat{\mu}+\lambda^{l e b}+\delta_{b} \ll \nu$ and denote by $\hat{h}$ the Radon Nikodym derivative $d \hat{\mu}=\hat{h} d \nu$. Let us define $\rho^{[a, b]}$ on $\mathbb{L}^{1}([a, b], \nu)$ by

$$
\rho^{[a, b]}(h):=\left\{\begin{array}{ll}
\rho(h d \nu) & \text { if } h \geq 0, \int h d \nu=1 \\
+\infty & \text { else }
\end{array} .\right.
$$

Since $\rho$ is $\|\cdot\|$-continuous and monotone with respect to the cone $\mathcal{K}^{1}$, it follows that $\rho^{[a, b]}$ is $\sigma\left(\mathbb{L}^{1}([a, b], \nu), \mathbb{L}^{\infty}([a, b], \nu)\right)$-lower semicontinuous, and monotone with respect to the cone $\mathcal{K}(\nu)$. Hence, applying Lemmata 2.16 and 2.26, there exists $u \in \mathcal{K}^{\circ}(\nu)$ such that

$$
-\int u \hat{h} d \nu>\sup _{\mu \in \mathcal{A}_{[a, b]}^{m} \cap \mathcal{M}_{1}([a, b], \nu)}-\int u d \mu .
$$

Due to Lemma 2.27 and the dominated convergence theorem, there is a continuous nondecreasing function $\hat{u} \in C([a, b])$ satisfying

$$
-\int \hat{u} d \hat{\mu}>\sup _{\mu \in \mathcal{A}_{[a, b]}^{m} \cap \mathcal{M}_{1}([a, b], \nu)}-\int \hat{u} d \mu=\alpha_{\min }^{[a, b]}(\hat{u}, m)
$$

where the last equality follows from Lemma 2.28. This shows (2.49). 


\section{Robust Representation of Risk Orders}

Step 2. For any $x \in \mathbb{R}$, we define $u(x):=-\rho\left(\delta_{x}\right)$. By monotonicity with respect to the first stochastic order it follows that $u$ is nondecreasing. Hence, by affinity of $\rho$ we derive $\rho(\mu)=-\int u d \mu$ for any simple probability distribution $\mu=\sum_{n=1}^{N} \alpha_{n} \delta_{x_{n}}$. For arbitrary $\mu \in \mathcal{M}_{1, c}$ with support in $[a, b] \subset I$ big enough according to Lemma 2.27 , there exists a sequence of simple probability distributions $\mu_{k}$ having support in $[a, b]$, converging in the $\sigma\left(\mathcal{M}_{1}([a, b]), C([a, b])\right)$-topology to $\mu$, and such that $\mu_{k}$ dominates $\mu$ in the first stochastic order. Thus, since both $\rho$ and $\mu \mapsto-\int u d \mu$ are $\sigma\left(\mathcal{M}_{1}([a, b]), C([a, b])\right)$ lower semicontinuous and monotone with respect to the first stochastic order it follows that

$$
\rho(\mu)=\lim _{k \rightarrow \infty} \rho\left(\mu_{k}\right)=\lim _{k \rightarrow \infty}-\int u d \mu_{k}=-\int u d \mu
$$

and the proof is completed. 


\section{Illustrative Settings}

The following sections address each setting introduced in the Section 1.1 in its particularity and illustrates how to consider risk under different perspectives with some concrete examples.

\subsection{Random Variables}

In the context of Section 2.1, recall that for the $\|\cdot\|_{\infty}$-topology on $\mathbb{L}^{\infty}$ the normalized polar cone with respect to $\pi=1$ is $\mathcal{K}_{1}^{\circ}=\mathcal{M}_{1, f}(P)$. However, the space $\mathcal{M}_{1, f}(P)$ is for many reasons not desirable; non- $\sigma$-additive measures do not have a density and are moreover truly not common. Even on the real line they can at most be constructed implicitly using the axiom of choice. We want therefore stronger representations in terms of $\sigma$-additive measures. This can be done by considering the weak ${ }^{*}$-topology $\sigma\left(\mathbb{L}^{\infty}, \mathbb{L}^{1}\right)$ on $\mathbb{L}^{\infty}$ instead of the $\infty$-norm. In that case, the normalized polar cone is $\mathcal{K}_{1}=\mathcal{M}_{1}(P)$. A condition to ensure the $\sigma\left(\mathbb{L}^{\infty}, \mathbb{L}^{1}\right)$-lower semicontinuity of quasiconvex functions is the so called Fatou property.

Definition 3.1 (Fatou Property). A set $\mathcal{B} \subset \mathcal{X}$ is Fatou closed if for any $\|\cdot\|_{\infty^{-}}$ bounded sequence $X_{n} \in \mathcal{B}$ converging $P$-almost surely to $X \in \mathcal{X}$, it follows that $X \in \mathcal{B}$. Consequently,

- a risk order $\succcurlyeq$ on $\mathcal{X} \subset \mathbb{L}^{\infty}$ has the Fatou property if for any $Y \in \mathcal{X}, \mathcal{L}(Y)$ is Fatou closed.

- a risk measure $\rho: \mathcal{X} \rightarrow[-\infty,+\infty]$ has the Fatou property if for any $\|\cdot\|_{\infty}$-bounded sequence $X_{n} \in \mathcal{X}$ converging $P$-almost surely to $X \in \mathcal{X}$ holds

$$
\liminf _{n \rightarrow+\infty} \rho\left(X_{n}\right) \geq \rho(X) .
$$

- a risk acceptance family $\mathcal{A}=\left(\mathcal{A}^{m}\right)_{m \in \mathbb{R}}$ has the Fatou property if $\mathcal{A}^{m}$ is Fatou closed for any $m \in \mathbb{R}$.

Remark 3.2. It is clear that a risk measure $\succcurlyeq$ has the Fatou property if and only if for any $X, Y \in \mathcal{X}$ and any $\|\cdot\|_{\infty}$-bounded sequence $X_{n}$ converging $P$-almost surely to $X$ holds

$$
Y \succcurlyeq X_{n} \text { for all } n \quad \Longrightarrow \quad Y \succcurlyeq X \text {. }
$$

It is also clear that a risk measure $\rho$ has the Fatou property if the corresponding risk acceptance family $\mathcal{A}$ has the Fatou property and vice versa. 


\section{Illustrative Settings}

Finally, if $\rho$ has the Fatou property, then so do $\succcurlyeq$. As for the lower semicontinuity, it is not clear that the converse is true. However, this will be the case in the sequel, since the Fatou property will imply the relative $\sigma\left(\mathbb{L}^{\infty}, \mathbb{L}^{1}\right)$-lower semicontinuity.

As for the convex set $\mathcal{X}$, it turns out in the study of stochastic kernels below that it is usefull to consider the strip

$$
\mathcal{X}=(A, B):=\left\{X \in \mathbb{L}^{\infty} \mid a<\operatorname{ess} \inf X \leq \operatorname{ess} \sup X<b\right\}
$$

for some $-\infty \leq a<b \leq+\infty$. On this convex set we obtain the following theorem.

Theorem 3.3. Any $\|\cdot\|_{\infty}$-lower semicontinuous risk measure $\rho:(A, B) \rightarrow[-\infty,+\infty]$ corresponding to a separable $\|\cdot\|_{\infty}$-lower semicontinuous risk order $\succcurlyeq$ has a robust representation

$$
\rho(X)=\sup _{Q \in \mathcal{M}_{1, f}(P)} R\left(Q, E_{Q}[-X]\right), \quad X \in(A, B),
$$

for a unique maximal risk function $\left.R: \mathcal{M}_{1, f}(P) \times\right] a, b[\rightarrow[-\infty, \infty]$, jointly quasiconcave, with a uniform asymptotic minimum ${ }^{1}$, and such that $R^{+}$is upper semicontinuous in the first argument.

If $\rho$ has the Fatou property, then $\rho$ is $\sigma\left(\mathbb{L}^{\infty}, \mathbb{L}^{1}\right)$-lower semicontinuous and the same statement as for the $\|\cdot\|_{\infty}$-lower semicontinity holds up to the fact that one can replace $\mathcal{M}_{1, f}(P)$ by $\mathcal{M}_{1}(P)$ in the robust representations (3.2).

Finally, if a separable risk order $\succcurlyeq$ on $(A, B)$ has the Fatou property, then $\succcurlyeq$ is $\sigma\left(\mathbb{L}^{\infty}, \mathbb{L}^{1}\right)$-lower semicontinuous and therefore by Proposition 2.2, there exists some $\sigma\left(\mathbb{L}^{\infty}, \mathbb{L}^{1}\right)$-lower semicontinuous risk measure $\rho$ corresponding to $\succcurlyeq$;

From the robust representation, we see as mentioned in the introduction, that risk orders on the level of random variables can be interpreted a model risk since a prudent approach is taken with respect to different probability models.

The case where $(A, B)=\mathbb{L}^{\infty}$ and $\rho$ is a cash additive risk measure was already extensively studied, see [Föllmer and Schied, 2004] and the references therein. The case where $(A, B)=\mathbb{L}^{\infty}$ and $\rho$ is a only cash subadditive was studied in [Cerreia-Vioglio et al., 2010]. They do not address however the Fatou property implications. The case where $(A, B)$ is a strict subset of $\mathbb{L}^{\infty}$ is in the context of the Fatou property particularly involved and new.

Proof. In the following, $\mathcal{T}$ denotes a topology on $\mathbb{L}^{\infty}$ and $\bar{A}$ denotes the $\mathcal{T}$-closure of a set $A \subset \mathbb{L}^{\infty}$. For the risk acceptance family $\mathcal{A}$ of a risk measure $\rho$, define the family

$$
\hat{\mathcal{A}}^{m}=\bigcap_{n>m} \overline{\mathcal{A}^{n}+\mathbb{L}_{+}^{\infty}}, \quad m \in \mathbb{R} .
$$

Since, $\mathcal{A}$ is a risk acceptance family on $\mathcal{X}$ it is straightforward to check that $\hat{\mathcal{A}}$ is a risk acceptance family on $\mathcal{V}$.

\footnotetext{
${ }^{1}$ Since $R(Q, \cdot)$ is defined on $] a, b\left[\right.$, this means $\lim _{s \searrow a} R(Q, s)=\lim _{s \searrow a} R\left(Q^{\prime}, s\right)$ for any $Q, Q^{\prime} \in$ $\mathcal{M}_{1, f}(P)$.
} 
Step 1: $\mathcal{T}$ is the $\|\cdot\|_{\infty}$-topology, and $\rho$ is $\mathcal{T}$-lower semicontinuous. In this case, $(A, B)$ is an open set of $\mathbb{L}^{\infty}$, and therefore, by way of Theorem $2.19, \hat{\mathcal{A}}$ coincides with $\mathcal{A}$ and defines the maximal $\mathcal{T}$-lower semicontinuous risk measure $\hat{\rho}$ which coincides with $\rho$.

Step 2: $\mathcal{T}$ is the $\sigma\left(\mathbb{L}^{\infty}, \mathbb{L}^{1}\right)$-topology, and $\rho$ is $\mathcal{T}$-lower semicontinuous. Here, $(A, B)$ is neither closed nor open in $\mathcal{T}$-topology. Let us prove, however, that the restriction of $\hat{\mathcal{A}}$ to $(A, B)$ is equal to $\mathcal{A}$ since it shows that the $\mathcal{T}$-lower semicontinuous $\hat{\rho}$ is an extension of $\rho$ on $\mathcal{V}$. It is obviously enough to show that $\overline{\mathcal{A}^{m}+\mathbb{L}_{+}^{\infty}} \cap(A, B)=\mathcal{A}^{m}$ for any $m \in \mathbb{R}$. If $b=+\infty$, then $\overline{\mathcal{A}^{m}+\mathbb{L}_{+}^{\infty}} \cap(A, B)=\overline{\mathcal{A}^{m}}$ and by the Krein-Šmulian it is then equal to $\mathcal{A}^{m}$ because it is Fatou closed. Suppose now that $b<+\infty$. The inclusion " $\supset$ " is clear. Let us show the other inclusion. Denote by $B_{K}=\left\{X \in \mathbb{L}^{\infty} \mid-K \leq X \leq K\right\}$ for $K>0$. Firstly, we show that $\bigcup_{K>0} \overline{\left(\mathcal{A}^{m}+\mathbb{L}_{+}^{\infty}\right) \cap B_{K}}$ is $\mathcal{T}$-closed. From Krein-Šmulian theorem, it is enough to show that $\bigcup_{K>0} \overline{\left(\mathcal{A}^{m}+\mathbb{L}_{+}^{\infty}\right) \cap B_{K}} \cap B_{K_{0}}$ is $\mathcal{T}$-closed for any $K_{0}>0$. Applying Lemma 2.20, it follows that

$$
\bigcup_{K>0} \overline{\left(\mathcal{A}^{m}+\mathbb{L}_{+}^{\infty}\right) \cap B_{K}} \cap B_{K_{0}}=\bigcup_{K>0} \overline{\left(\mathcal{A}^{m}+\mathbb{L}_{+}^{\infty}\right) \cap B_{K} \cap B_{K_{0}}}=\overline{\left(\mathcal{A}^{m}+\mathbb{L}_{+}^{\infty}\right) \cap B_{K_{0}}}
$$

and therefore $\bigcup_{K>0} \overline{\left(\mathcal{A}^{m}+\mathbb{L}_{+}^{\infty}\right) \cap B_{K}}$ is $\mathcal{T}$-closed. Secondly, $\overline{\mathcal{A}^{m}+\mathbb{L}_{+}^{\infty}}$ is the smallest $\mathcal{T}$-closed set containing $\mathcal{A}^{m}+\mathbb{L}_{+}^{\infty}$ and thus $\overline{\mathcal{A}^{m}+\mathbb{L}_{+}^{\infty}}=\bigcup_{K>0} \overline{\left(\mathcal{A}^{m}+\mathbb{L}_{+}^{\infty}\right) \cap B_{K}}$. Thirdly, from the previous equality, for any $X \in \overline{\mathcal{A}^{m}+\mathbb{L}_{+}^{\infty}} \cap(A, B)$ there exist sequences $X_{n}=R_{n}+Z_{n}$ such that $R_{n} \in \mathcal{A}^{n}, Z_{n} \in \mathbb{L}_{+}^{\infty}$ and $X_{n}$ converges $P$-almost surely to $X$. Indeed, there exists some $b<K_{0}$ such that $X \in \overline{\mathcal{A}^{m}+\mathbb{L}_{+}^{\infty}} \cap B_{K_{0}}$ and we can use [Föllmer and Schied, 2004, Lemma A.64]. Modifying $Z_{n} \in \mathbb{L}_{+}^{\infty}$ adequately, we can suppose without lost of generality that $R_{n} \geq-K_{0}-1$. Since $b<+\infty, R_{n}$ is uniformly bounded $P$-almost surely, and therefore, using [Föllmer and Schied, 2004, Lemma 1.61], we can assume up to a convex combinations in $\operatorname{conv}\left\{R_{k} \mid k \geq n\right\}$, that $R_{n}$ and as a consequence $Z_{n}$ converge $P$-almost surely respectively to some $R$ and $Z$. Fourthly, if $R \in(A, B)$, then, because of the Fatou property, $R \in \mathcal{A}^{m}$, and so, from the monotonicity, $X \in \mathcal{A}^{m}$. Finally, if $R \notin(A, B)$, suppose then that $X \notin \mathcal{A}^{m}$. In that case, there exists some $\varepsilon>0$ such that $X+\varepsilon \in(A, B)$ and $X+\varepsilon \notin \mathcal{L}(Y)$. Indeed, use the Fatou property on $X+1 / n$ for any $n>0$ to get a contradiction. However, it means that $X=R+\varepsilon+Z$ with $R_{n}+\varepsilon \in \mathcal{A}^{m}$ and $R+\varepsilon \in \mathcal{A}^{m}$ because of the monotonicity and the Fatou property. Thus $X \in \mathcal{A}^{m}$ contradicting our supposition that $X \notin \mathcal{A}^{m}$. We deduce then that $\hat{\rho}$ is an extension of $\rho$.

Step 3: In the setting of Step 1, corresponding to $\hat{\rho}$, there exists by Theorem 2.8 a unique maximal risk function $\hat{R}: \mathcal{M}_{1, f}(P) \times \mathbb{R} \rightarrow[-\infty,+\infty]$ in $\mathcal{R}_{1}^{\max }$ such that

$$
\rho(X)=\sup _{Q \in \mathcal{M}_{1, f}(P)} \hat{R}\left(Q, E_{Q}[-X]\right), \quad X \in(A, B) .
$$

In the setting of Step 2, one can replace $\mathcal{M}_{1, f}(P)$ by $\mathcal{M}_{1}(P)$. Consider now some function $\left.R: \mathcal{M}_{1, f}(P) \times\right] a, b[\rightarrow[-\infty,+\infty]$, jointly quasiconcave, with a uniform asymptotic 


\section{Illustrative Settings}

minimum, such that $R^{+}$is upper semicontinuous in the first argument, and

$$
\rho(X)=\sup _{Q \in \mathcal{M}_{1, f}(P)} R\left(Q, E_{Q}[-X]\right), \quad X \in(A, B) .
$$

Extend then $R$ outside $] a, b[$ in the following way:

$$
\left\{\begin{array}{ll}
R(Q, s)=\lim _{s^{\prime} \searrow^{a}} R\left(Q, s^{\prime}\right) & \text { for } s \leq a \\
R(Q, b)=\lim _{s^{\prime} \nearrow_{b}} R\left(Q, s^{\prime}\right) & \\
R(Q, s)=+\infty & \text { for } s>b
\end{array} .\right.
$$

Straightforward inspection shows that this extended $R$ belongs to $\mathcal{R}_{1}^{\max }$ and that relation (3.3) holds for this extended $R$ instead of $\hat{R}$. The uniqueness of $\hat{R}$ ensures then the uniqueness in terms of $R$. The same argumentation holds in the case of $\mathcal{M}_{1}(P)$ instead of $\mathcal{M}_{1, f}(P)$.

Step 4: $\mathcal{T}$ is the $\sigma\left(\mathbb{L}^{\infty}, \mathbb{L}^{1}\right)$-topology. Suppose here that $\succcurlyeq$ is a separable risk order fulfilling the Fatou property. In order to show that $\succcurlyeq$ is $\mathcal{T}$-lower semicontinuous, it is sufficient to show that $\mathcal{L}(Y)$ is relatively closed for any $Y \in(A, B)$. To do so we show that $\overline{\mathcal{L}(Y)+\mathbb{L}_{+}^{\infty}} \cap(A, B)=\mathcal{L}(Y)$. But this follows exactly the same argumentation as in the second step.

Under a directional closedness assumption on the risk order $\succcurlyeq$, we obtain an automatic continuity result on the convex set $(A, B)$.

Proposition 3.4. Let $\succcurlyeq$ be a separable risk order on $(A, B)$. If for any $X, Y, Z \in(A, B)$ with $X \succcurlyeq Y$, the set

$$
\{\lambda \in[0,1] \mid X \succcurlyeq \lambda Y+(1-\lambda) Z\},
$$

is closed in $[0,1]$, then the risk order $\succcurlyeq i s\|\cdot\|_{\infty}$-lower semicontinuous.

Remark 3.5. In the case that $(A, B)=\mathbb{L}^{\infty}$ and that $\rho$ is a $\|\cdot\|_{\infty}$-lower semicontinuous cash additive risk measure, it follows from Proposition 2.17 and Theorem 3.3 that it has a robust representation of the form

$$
\rho(X)=\sup _{Q \in \mathcal{M}_{1, f}(P)}\left\{E_{Q}[-X]-\alpha_{\min }(Q, 0)\right\}, \quad X \in(A, B) .
$$

for a unique minimal penalty function $\alpha_{\min }$.

Proof. Note that if $(A, B)=\mathbb{L}^{\infty}$ this is a direct application of Theorem 2.22 since any risk order monotone with respect to the "greater than $P$-almost sure" preorder is automatically $\pi$-bounded.

We will show that $\mathcal{L}(X)$ is relatively closed in $(A, B)$ for any $X \in(A, B)$. Suppose that $\mathcal{L}(X) \neq(A, B)$ and $\mathcal{L}(X) \neq \emptyset$ otherwise it is trivial. Take $X \in(A, B)$ and define $\mathcal{A}^{X}=\mathcal{L}(X)+\mathbb{L}_{+}^{\infty}$. Our assumption on $\mathcal{L}(X)$ implies that $\mathcal{A}^{X} \neq \emptyset$ and by monotony 
of $\succcurlyeq$ that $\mathcal{A}^{X} \neq \mathbb{L}^{\infty}$. The functional

$$
\rho^{X}(Y)=\inf \left\{n \in \mathbb{R} \mid Y+n \in \mathcal{A}^{X}\right\}, \quad Y \in \mathbb{L}^{\infty},
$$

is by construction a cash additive risk measure. From $\mathcal{A}^{X} \neq \emptyset$ or $\mathcal{A}^{X} \neq \mathbb{L}^{\infty}$ and the fact that $\mathcal{A}^{X}$ is monotone, follows that $\rho^{X}$ is real valued. By [Borwein, 1987, Theorem 2.2], $\rho^{X}$ is continuous and therefore $\tilde{A}^{X}=\left\{Y \in \mathbb{L}^{\infty} \mid \rho^{X}(Y) \leq 0\right\}$ is closed. It remains to show that $\tilde{\mathcal{A}}^{X} \cap(A, B)=\mathcal{L}(X)$. To do so, we adapt an argumentation from [Föllmer and Schied, 2004, Chap 4.1]. From the definition of $\rho^{X}$ it is clear that $\tilde{\mathcal{A}}^{X} \supset \mathcal{A}^{X}$. Since, by monotony, $\mathcal{A}^{X} \cap(A, B)=\mathcal{L}(X)$, it follows that $\tilde{\mathcal{A}}^{X} \cap(A, B) \supset \mathcal{L}(X)$. Conversely, chose $Y \in(A, B) \backslash \mathcal{L}(X)$ and pick some $m \in] \max \{\operatorname{ess} \sup Y$, ess $\sup X\}, b[$. By monotonicity, $m \in \mathcal{L}(X)$. Furthermore, since

$$
\{\lambda \in[0,1] \mid X \succcurlyeq \lambda m+(1-\lambda) Y\}
$$

is a non empty closed set in $[0,1]$ and does not contains 0 , there exists $\varepsilon>0$ such that $\varepsilon m+(1-\varepsilon) Y \notin \mathcal{L}(X)$. Together with the cash additivity of $\rho^{X}$, holds

$$
0 \leq \rho^{X}(\varepsilon m+(1-\varepsilon) Y)=-\varepsilon m+\rho^{X}((1-\varepsilon) Y)
$$

On the other hand, the monotonicity of $\rho^{X}$ applied to $Y \leq(1-\varepsilon) Y+\varepsilon \operatorname{ess} \sup Y$ yields

$$
\rho^{X}(Y) \geq \rho^{X}((1-\varepsilon) Y)-\varepsilon \operatorname{ess} \sup Y \geq \varepsilon(m-\operatorname{ess} \sup Y)>0,
$$

from which follows that $Y \notin \tilde{\mathcal{A}}^{X}$, and therefore $\tilde{\mathcal{A}}^{X} \cap(A, B)=\mathcal{L}(X)$. This proves that $\mathcal{L}(X)$ is relatively closed in $(A, B)$.

Let us now present how it applies to some concrete examples which all fulfill the Fatou property due to adequate continuity assumptions.

Example 3.6. The optimized certainty equivalent introduced in Example 1.25 has a robust representation of the form

$$
\rho(X):=-\sup _{m \in \mathbb{R}}\{m+E[u(X-m)]\}=\sup _{Q \in \mathcal{M}_{1}(P)}\left\{E_{Q}[-X]-\alpha_{\min }(Q)\right\}, \quad X \in \mathbb{L}^{\infty},
$$

where the minimal penalty function is the so called $\varphi$-divergence given by

$$
\alpha_{\min }(Q)=E\left[\varphi\left(\frac{d Q}{d P}\right)\right], \quad Q \in \mathcal{M}_{1}(P) .
$$

Here, $\varphi$ is the conjugate of $s \mapsto-u(-s)$. This example entails in particular the $A V @ R_{q}$ introduced in Example 1.25 if $u(s)=1 / q \min (s, 0)$, since $\varphi(s)=0$ if $0 \leq s \leq 1 / q$ and $\varphi(s)=+\infty$ elsewhere. The entropic risk measure presented in Example 1.25 is also a special case for $u(s)=1-e^{-s}$ since $\varphi(s)=s \ln (s)+1-s$ yielding to the relative 


\section{Illustrative Settings}

entropy

$$
\alpha_{\min }(Q)=E\left[\frac{d Q}{d P} \ln \left(\frac{d Q}{d P}\right)\right], \quad Q \in \mathcal{M}_{1}(P) .
$$

These results can be found in [Ben-Tal and Teboulle, 2007].

Finally, the shortfall risk measure introduced in Example 1.13, in the case where $l$ is well-defined on $\mathbb{R}$, has a robust representation of the form

$$
\rho(X)=\sup _{Q \mathcal{M}_{1}(P)}\left\{E_{Q}[-X]-\alpha_{\min }(Q)\right\}, \quad X \in \mathbb{L}^{\infty},
$$

where the minimal penalty function is given by

$$
\alpha_{\min }(Q)=\inf _{\lambda>0} \frac{1}{\lambda}\left(E[\varphi(\lambda d Q / d P)]-c_{0}\right), \quad Q \in \mathcal{M}_{1}(P),
$$

whereby $\varphi(s)=-l^{*}(-s)$, [see Föllmer and Schied, 2004, Theorem 4.106].

We address now the robust representation of the certainty equivalent in Example 1.8.

Example 3.7 (Certainty Equivalent). Recall that the certainty equivalent is given by

$$
\rho(X)=\left\{\begin{array}{ll}
l^{-1}(E[l(-X)]) & X>a_{0} P \text {-almost surely } \\
+\infty & \text { otherwise }
\end{array} \quad X \in \mathbb{L}^{\infty}\right.
$$

where $l: I=]-\infty,-a_{0}\left[\rightarrow \mathbb{R}\right.$ is a nondecreasing ${ }^{2}$ upper semicontinuous concave function and $a_{0} \in[-\infty,+\infty[$. We present hereafter a method to compute the robust representation, and assume for simplicity that $l$ is differentiable on its domain. Using relation (2.13) from Proposition 2.13, the penalty function is given for any $Q \in \mathcal{M}_{1}(P)$ and $m \in \mathbb{R}$ by

$$
\begin{aligned}
\alpha_{\min }(Q, m)=\sup _{\left\{X \in \mathbb{L}^{\infty} \mid E[l(-X)] \geq l^{+}(m)\right\}} & E_{Q}[-X] \\
= & \sup _{X \in \mathbb{L}^{\infty}} E\left[-\frac{d Q}{d P} X-\frac{1}{\beta}\left(l(-X)-l^{+}(m)\right)\right]
\end{aligned}
$$

for some Lagrange multiplier $\beta:=\beta(Q, m)>0$. The first order condition yields

$$
-\frac{d Q}{d P}+\frac{1}{\beta} l^{\prime}(-\hat{X})=0 \quad \Longleftrightarrow \quad \hat{X}=-h\left(\beta \frac{d Q}{d P}\right),
$$

for the optimizer $\hat{X}$ of (3.8) where $h$ denotes a pseudo inverse of $l^{\prime}$ as it is nonincreasing. Therefore, $\beta$ is determined through the equation

$$
E\left[l\left(h\left(\beta \frac{d Q}{d P}\right)\right)\right]=l^{+}(m)
$$

\footnotetext{
${ }^{2}$ In the first section we assumed for simplicity that $l$ is increasing. We can however take nondecreasing $l$ which might then be constant from some point. Since $l$ is lower semicontinuous it is right-continuous, and we take as $l^{-1}$ the left inverse which is then nondecreasing concave and lower semicontinuous, see Proposition 2.13.
} 
Plugging the optimizer $\hat{X}$ in $(3.8)$ yields

$$
\alpha_{\min }(Q, m)=E_{Q}\left[h\left(\beta(Q, m) \frac{d Q}{d P}\right)\right]
$$

We subsequently list closed form solutions for specific $u$. We explicit the computations only for the first one, the others are left to the reader.

- Quadratic Functional: Suppose that $l(s)=s^{2} / 2-s$ for $s \geq-1$ and $l(s)=$ $-1 / 2$ elsewhere. This function corresponds to a monotone version of the meanvariance risk measure of MARKowitz [1952]. In this case, $l^{-1}(t)=\sqrt{2 t+1}-1$ if $t \leq-1 / 2$ and $-\infty$ elsewhere, therefore

$$
\rho(X):= \begin{cases}\sqrt{2 E\left[\frac{X^{2}}{2}-X\right]+1}-1 & \text { if } E\left[-X+\frac{X^{2}}{2}\right] \geq-\frac{1}{2}, \quad X \in \mathbb{L}^{\infty} . \\ -\infty & \text { else }\end{cases}
$$

Direct computation yields $l^{\prime}(s)=s+1$ for $s \geq-1$ and $l^{\prime}(s)=0$ elsewhere, $h(s)=s-1$ for $s \geq 0$ and $h(s)=+\infty$ elsewhere implying $l(h(s))=1 / 2\left(s^{2}-1\right)$ for $s \geq 0$ and $l(h(s))=-1 / 2$ elsewhere. Thus, relation (3.9) implies

$$
\beta(Q, m)=\frac{\sqrt{2 l(m)+1}}{\|d Q / d P\|_{\mathbb{L}^{2}}} .
$$

Plugging into relation (3.10) yields

$$
\begin{aligned}
\alpha_{\min }(Q, m) & =\|d Q / d P\|_{\mathbb{L}^{2}} \sqrt{2 l(m)+1}-1 \\
& = \begin{cases}(1+m)\|d Q / d P\|_{\mathbb{L}^{2}}-1 & \text { for } m \geq-1 \\
-1 & \text { else }\end{cases}
\end{aligned}
$$

Inverting yields

$$
R(Q, s)=\frac{s+1}{\|d Q / d P\|_{\mathbb{L}^{2}}}-1
$$

if $s>1$, and $R(Q, s)=-\infty$ elsewhere, and therefore

$$
\rho(X)=\sup _{Q \in \mathcal{M}_{1}(P)}\left\{\frac{E_{Q}[-X]+1}{\|d Q / d P\|_{\mathbb{L}^{2}}}-J\left(E_{Q}[-X]\right)\right\}, \quad X \in \mathbb{L}^{\infty},
$$

whereby $J(s)=1$ if $s>1$ and $J(s)=+\infty$ elsewhere.

- Exponential Function: If $l(s)=e^{s}-1$, then

$$
\rho(X):=\ln \left(E\left[e^{-X}\right]\right)=\sup _{Q \in \mathcal{M}_{1}(P)}\left(E_{Q}[-X]-E\left[\frac{d Q}{d P} \log \left(\frac{d Q}{d P}\right)\right]\right), \quad X \in \mathbb{L}^{\infty} .
$$


- Logarithm Function: If $l(s)=-\ln (-s)$ for $s<0$, then

$$
\rho(X):=-\exp (E[\ln (X)])=\sup _{Q \in \mathcal{M}_{1}(P)}\left\{\frac{E_{Q}[-X]}{\exp \left(E\left[\ln \left(\frac{d Q}{d P}\right)\right]\right)}\right\}, \quad X \in \mathbb{L}^{\infty} .
$$

- Power Functions: If $l(s)=-(-s)^{1-\gamma} /(1-\gamma)$ for $s \geq 0$ and $0<\gamma<1$, we obtain

$$
\rho(X)=\sup _{Q \in \mathcal{M}_{1}(P)}\left\{E\left[(d Q / d P)^{\frac{\gamma-1}{\gamma}}\right]^{\frac{\gamma}{1-\gamma}} E_{Q}[-X]\right\}, \quad X \in \mathbb{L}^{\infty} .
$$

To assess the economic index of riskiness, we use the same technique.

Example 3.8 (Economic Index of Riskiness). For the definition and notations, we refer to Example 1.13. The risk acceptance family is given for $m>0$ by

$$
\mathcal{A}^{m}=\left\{X \in \mathbb{L}^{\infty} \mid E[l(-X / m)] \leq c_{0}\right\}
$$

which is closed since $l$ is lower semicontinuous, and for $m \leq 0$ by $\mathcal{A}^{m}=\mathbb{L}_{+}^{\infty}$. Applying the same technique as for the certainty equivalent yields

$$
\alpha_{\min }(Q, m)=\sup _{X \in \mathbb{L}^{\infty}} E\left[-\frac{d Q}{d P} X+\frac{1}{\beta}\left(c_{0}-l(-X / m)\right)\right], \quad Q \in \mathcal{M}_{1}(P) .
$$

The optimizer is here given by $\hat{X}=-m h(\beta m d Q / d P)$ where $h$ is a pseudo inverse of $l^{\prime}$. The lagrange multiplier is given by $E[l(h(\beta m d Q / d P))]=c_{0}$, showing that

$$
\alpha_{\min }(Q, m)=E_{Q}[m h(\beta m d Q / d P)], \quad m>0 .
$$

In the case of Aumann And Serrano [2008] where $l(s)=e^{s}$ holds

$$
\rho(X)=\sup _{Q \in \mathcal{M}_{1}(P)} \frac{E_{Q}[-X]}{E_{Q}\left[\ln \left(c_{0} d Q / d P\right)\right]}, \quad X \in \mathbb{L}^{\infty} .
$$

In the case of Foster And Hart [Forthcoming] where $l(s)=\ln (1-s)$ holds

$$
\rho(X)=\sup _{Q \in \mathcal{M}_{1}(P)} \frac{E_{Q}[-X]}{E\left[\exp \left(c_{0}+E[\ln (d Q / d P)]\right)\right]-1}, \quad X \in \mathbb{L}^{\infty},
$$

and both are clearly positive homogeneous, due to Proposition 2.17 .

We finally consider the examples of numéraire uncertainty and liquidity costs.

Example 3.9 (Liquidity Costs). Let $\rho$ be the risk measure corresponding to the risk acceptance family for the liquidity costs of Example 1.30. The minimal penalty function 
is here given by

$$
\begin{aligned}
\alpha_{\min }(Q, m)=\sup _{X \in \mathcal{A}^{m}} E_{Q}[-X]=\sup _{X^{\prime} \in \mathcal{A}^{0}} E_{Q}\left[-X^{\prime}\right]+(m-c(m)) & =\alpha_{\min }(Q, 0)+(m-c(m)) .
\end{aligned}
$$

From the inversion of $\alpha_{\min }(Q, m)$ follows

$$
\rho(X)=\sup _{Q \in \mathcal{M}_{1}(P)} \varphi^{-1}\left(E_{Q}[-X]-\alpha_{\min }(Q, 0)\right), \quad X \in \mathbb{L}^{\infty},
$$

where $\varphi(m)=m-c(m)$.

\subsection{Probability Distributions}

We now consider the cornerstone setting of probability distributions with compact support. Recall that $\mathcal{M}_{1, c}$ is a convex subset of the vector space $c a_{c}$ and that the first stochastic order and the second stochastic orders are induced respectively by the polar cones

$$
\begin{aligned}
& \mathcal{K}^{1, \circ}=\{u \in C \mid u \text { is nondecreasing }\}, \\
& \mathcal{K}^{2, \circ}=\{u \in C \mid u \text { is nondecreasing and concave }\} .
\end{aligned}
$$

Proposition 3.10. Let $\succcurlyeq$ be a $\sigma\left(c a_{c}, C\right)$-lower semicontinuous risk order on $\mathcal{M}_{1, c}$ monotone with respect to the $i$-th stochastic order for $i$ equal to either 1 or 2 . Then any corresponding lower semicontinuous risk measure $\rho$ has the robust representation

$$
\rho(\mu)=\sup _{u \in \mathcal{K}^{i, o}} R\left(u,-\int u d \mu\right), \quad \mu \in \mathcal{M}_{1, c}
$$

for a unique $R \in \mathcal{R}^{\max }$.

This robust representation justifies the term distributional risk as for the risk orders on the level of lotteries, since the prudent approach is here taken with respect to non decreasing continuous functions which can be seen as distributions on the real line.

Remark 3.11. For affine risk orders which are monotone with respect to the first stochastic order, the lower semicontinuity requirement is not necessary. Indeed, under the assumptions of Theorem 2.24 they are automatically $\sigma\left(c a_{c}, C\right)$-lower semicontinuous.

Proof. The proof is a direct consequence of the Theorems 2.7 and 2.19 , since $\mathcal{M}_{1, c}$ is closed in $c a_{c}$. Moreover, even if $\mathcal{M}_{1, c}$ is a convex subset of $c a_{c}$ and the uniqueness of the maximal risk function refers to the extension $\hat{\rho}$ of $\rho$ in Theorem 2.19, a similar argumentation as in Theorem 3.3 shows that the unique risk function $R(u, \cdot)$ for $\rho$ has to coincide with $\hat{R}(u, \cdot)$ on the interval $\left\{-\int u d \mu \mid \mu \in \mathcal{M}_{1, c}\right\}$ which is in fact equal to $\mathbb{R}$. Therefore $R=\hat{R}$ and the uniqueness is proven. 


\section{Illustrative Settings}

Note that even if the first and the second stochastic order are not regular, it is enough to take the supremum in (3.16) over the "normalized" cone

$$
\mathcal{K}^{i, \circ}\left(x_{0}\right)=\left\{u \in \mathcal{K}^{i, \circ} \mid u\left(x_{0}\right)=0\right\}, \quad i=1,2
$$

for some $x_{0} \in I$. Indeed, any $u \in \mathcal{K}^{i, \circ}$ can be written as $u=\bar{u}+c$ for some $\bar{u} \in \mathcal{K}^{i, \circ}\left(x_{0}\right)$ and a constant $c \in \mathbb{R}$. Since $\alpha_{\min }(u, m)=\alpha_{\min }(\bar{u}, m)-c$, it follows that $R(u, s)=$ $R(\bar{u}, s+c)$ and therefore

$$
R\left(u,-\int u d \mu\right)=R\left(\bar{u},-\int u d \mu+c\right)=R\left(\bar{u},-\int \bar{u} d \mu\right)
$$

for all $\mu \in \mathcal{M}_{1, c}$.

Example 3.12 (Certainty equivalent). The certainty equivalent of a probability distribution introduced in Example 1.8 is continuous for any continuous increasing function $u: I \rightarrow \mathbb{R}$. It is already in its robust representation form, since

$$
\rho(\mu)=\sup _{\tilde{u} \in \mathcal{K}^{1, \circ}} R\left(\tilde{u},-\int \tilde{u} d \mu\right), \quad \mu \in \mathcal{M}_{1, c},
$$

where the maximal risk function is given by

$$
R(\tilde{u}, t)= \begin{cases}-\tilde{u}^{-1}(-t) & \text { if } \tilde{u}=u \\ \inf _{\mu \in \mathcal{M}_{1, c}} \rho(\mu) & \text { elsewhere }\end{cases}
$$

Example 3.13 (Value at Risk). Following the prescriptions of Basel II, "Value at Risk" is the central instrument used by banking institutions to assess their exposure to risk in a monetary way. Regardless of repeated critics, starting with ARTZNER ET AL. [1999], that it might penalize diversification since it is not quasiconvex, this measure instrument remains astonishingly resilient in the practice, even after the recent financial crisis. They are several arguments for the defense of the "Value at Risk"3, but the most recurrent one is that many persons think that it gives though some indications about risk. It is this strong but erroneous intuition we here want to study and try to explain.

The "Value at Risk" is defined for $q \in] 0,1[$ by

$$
V @ R_{q}(X)=\sup \{s \in \mathbb{R} \mid P[X+s \leq 0]>q\}, \quad X \in \mathbb{L}^{\infty} .
$$

This functional is cash additive and monotone, but not quasiconvex. From its definition, $V @ R_{q}$ depends only on the distribution of $X$, and can therefore be viewed ${ }^{4}$ on $\mathcal{M}_{1, c}$

\footnotetext{
${ }^{3}$ For instance, restricted to Gaussian risky assets, the "Value at Risk" is a convex risk measure.

${ }^{4}$ This has been done in [Weber, April 2006] for the case of monetary risk measures considered on the level of probability distributions.
} 
for $I=\mathbb{R}$, that is

$$
\left.\left.V @ R_{q}(\mu):=\sup \{s \in \mathbb{R} \mid \mu(]-\infty,-s]\right)>q\right\}=-F_{\mu}^{-1}(q), \quad \mu \in \mathcal{M}_{1, c} .
$$

where $F_{\mu}^{-1}$ is the right inverse of the nondecreasing distribution function $s \mapsto F_{\mu}(s):=$ $\mu(]-\infty, s])$. In fact, $V @ R_{q}(X)=V @ R_{q}(\mu)$ for $\mu=P_{X} \in \mathcal{M}_{1, c}$. On the level of probability distributions, $V @ R_{q}$ is monotone with respect to the first stochastic order. Moreover, for any risk level $m \in \mathbb{R}$, it follows from relation (2.13) that

$$
\begin{aligned}
\mathcal{A}^{m}=\{\mu & \left.\in \mathcal{M}_{1, c} \mid V @ R_{q}(\mu) \leq m\right\}=\left\{\mu \in \mathcal{M}_{1, c} \mid F_{\mu}^{-1}(q) \geq-m\right\} \\
& =\left\{\mu \in \mathcal{M}_{1, c} \mid q \geq F_{\mu}^{-}(-m)\right\}=\left\{\mu \in \mathcal{M}_{1, c} \mid q \geq \mu(]-\infty,-m[)\right\}
\end{aligned}
$$

which is a convex set. Therefore, $V @ R_{q}$ is a risk measure on $\mathcal{M}_{1, c}$.

Remark 3.14. The strong belief of the finance industry in the "Value at Risk" as a risk measure, is from this viewpoint truly founded, since it is indeed a risk measure on the level of probability distributions. It remains, however, a fundamental error to consider it as a reliable instrument to assess the risk of financial positions which are definitively random variable for which the pointwise diversification is wanted. So, even if this instrument is in principle a sound one, it is fundamentally misused in the wrong environment.

Further, according to [Aliprantis and Border, 2006, Corollary 15.6], the set

$$
\mathcal{A}^{m}=\left\{\mu \in \mathcal{M}_{1, c} \mid q \geq \mu(]-\infty,-m[)\right\},
$$

is $\sigma\left(c a_{c}, C\right)$-closed in $\mathcal{M}_{1, c}$, implying that $V @ R$ is a lower semicontinuous risk measure on $\mathcal{M}_{1, c}$. Due to Theorem 3.10, it admits then a robust representation. To compute the penalty function $\alpha_{\min }(u, m)=\sup _{\mu \in \mathcal{A}^{m}}-\int u d \mu$, we define $\mu_{t}:=q \delta_{t}+(1-q) \delta_{-m}$ which is in $\mathcal{A}^{m}$ since $F_{\mu_{t}}^{-}(-m) \leq q$ for all $t \in \mathbb{R}$. Then for any $u \in \mathcal{K}^{1, \circ}$

$$
\alpha_{\min }(u, m)=\sup _{\mu \in \mathcal{A}^{m}}-\int u d \mu=\lim _{t \rightarrow-\infty}-\int u d \mu_{t}=-q u(-\infty)-(1-q) u(-m) .
$$

Thus, for any $u \in \mathcal{K}^{1, \circ}$ bounded from below ${ }^{5}$ we get

$$
R(u, s)=-u^{-1}\left(\frac{s+q \inf u}{q-1}\right)
$$

where $u^{-1}$ is the right inverse of $u$ and therefore

$$
V @ R_{q}(\mu)=\sup _{u \in \mathcal{K}^{1, \circ}, \inf u>-\infty}-u^{-1}\left(\frac{-\int u d \mu+q \inf u}{q-1}\right) .
$$

${ }^{5}$ Otherwise, $R(u, \cdot) \equiv-\infty$. 


\section{Illustrative Settings}

\subsection{Consumption Streams}

Hereafter follows an insight on how our robust representation result can be applied in the context of preferences on consumption streams and in particular for the intertemporal utility functional of Hindy, HuAng, AND KrePs [1992]. Remind that the commodity space $\mathcal{C} \mathcal{S}_{+}$is the set of nondecreasing right-continuous functions $c:[0,1] \rightarrow \mathbb{R}$. In [Hindy et al., 1992], several reasons were given to consider an economical and mathematical sound topology which addresses coherently both continuity and jumps issues in consumption patterns. We refer to [Hindy et al., 1992] for a stimulating discussion about this. They show that a topology on $\mathcal{C S}:=\mathcal{C S} \mathcal{S}_{+}-\mathcal{C S}+$ fulfilling their requirements is the Orlicz topology generated by the Orlicz norm $\|\cdot\|_{\eta}$. For an element $c \in \mathcal{C} \mathcal{S}$, it is given by

$$
\|c\|_{\eta}=\inf \left\{m>0 \mid \int_{0}^{1} \eta\left(\left|c_{t}\right| / m\right) d t+\eta\left(\left|c_{1}\right| / m\right) \leq 1\right\},
$$

where $\eta=\mu^{-1}$ is some continuous concave increasing function $\mu:[0,+\infty[\rightarrow[0,+\infty[$ with $\mu(0)=0$ and $\lim _{t \rightarrow+\infty} \mu(t)=+\infty$, see [Hindy et al., 1992].

For this topology, the dual $\mathcal{C S}^{*}$ is

- either the set of Lipschitz functions $f:[0,1] \rightarrow \mathbb{R}$, if

$$
\lim _{t \rightarrow+\infty} \eta(t) / t=\alpha>0
$$

- or the set of absolute continuous function $f:[0,1] \rightarrow \mathbb{R}$ such that $f^{\prime}$ fulfills

$$
\lim _{\gamma \rightarrow 0+} \int_{0}^{1} \eta^{*}\left(\gamma\left|f^{\prime}(t)\right|\right) d t+\eta^{*}\left(\gamma\left|f^{\prime}(1)\right|\right)=0,
$$

where $\eta^{*}$ is the conjugate function of $\eta$, if

$$
\lim _{t \rightarrow 0+} \mu(t) / t=0 \quad \text { and } \quad \lim _{t \rightarrow+\infty} \mu(t) / t=+\infty .
$$

The preorder introduced in Section 1.1 is $\mathcal{C S}_{+}$with corresponding polar cone

$$
\mathcal{C S}_{+}^{\circ}=\left\{f \in \mathcal{C S}^{*} \mid f \geq 0\right\} .
$$

For further details we refer to [Hindy et al., 1992].

Proposition 3.15. Any lower semicontinuous risk measure $\rho$ of a lower semicontinuous risk order $\succcurlyeq$ on $\mathcal{C} \mathcal{S}_{+}$has a robust representation

$$
\rho(c)=\sup _{f \in \mathcal{C S}_{+}^{\circ}} R\left(f,-\int_{0}^{1} f(s) d c_{s}\right), \quad c \in \mathcal{C} \mathcal{S}_{+}
$$


for a unique maximal risk function $\left.\left.R: \mathcal{C S}_{+}^{\circ} \times\right]-\infty, 0\right] \rightarrow[-\infty,+\infty]$ which fulfills conditions (i), (ii), (iii) and (iv) of definition 2.6 restricted on $]-\infty, 0]$.

Here the robust representation shows that risk orders on the level of consumption streams is expressed in terms of discounting, and therefore justifies to interpret it as a discounting risk.

Proof. This is a direct application of the Theorems 2.7 and 2.19 since $\mathcal{C} \mathcal{S}_{+}$is a closed convex set. As for the uniqueness, we refer to the argumentation given for the Theorem 3.3 .

This robust representation gives a new perspective on the previously mentioned intertemporal utility functional. Considered as risk functional it is given by

$$
\rho(c)=-\int_{0}^{1} u\left(y_{t}+\int_{t-k_{1}(t)}^{t+k_{2}(t)} \theta(t, s) d c_{s}, t\right) d t, \quad c \in \mathcal{C S}_{+},
$$

for some parameter functions ${ }^{6} y, \theta, k_{1}$ and $k_{2}$ and a jointly measurable utility function $u: \mathbb{R}^{2} \rightarrow \mathbb{R}$ uniformly bounded from below. As for the term intertemporal utility functional, it means that the contribution of a commodity consumption to utility at time $t$ is not the "instant" consumption rate at this time, but a weighted average of the consumption in the time around $t$.

Proposition 3.16. The risk functional measure $\rho$ given by $(3.22)$ is lower semicontinuous if and only if $u$ is upper semicontinuous non decreasing and concave in its first component. In that case it has a robust representation of the form (3.21).

Proof. Let us consider first the sufficiency. Let $c_{n} \rightarrow c$ be a converging sequence of consumption streams in the Orlicz topology. The proof that $c \mapsto y^{(n)}+\int_{-k_{1}(\cdot)}^{\cdot+k_{2}(\cdot)} \theta(\cdot, s) d c_{s}$ is continuous in the Orlizc topology is done in Hindy et al. [1992]. The proof that it is lower semicontinuous follows from Lebegue's theorem and the upper semicontinuity of $u$. As $\theta$ is positive and $u$ increasing, the monotonicity is immediate. Finally, the concavity of $u$ implies the convexity of the intertemporal risk functional.

Conversely, the concavity of $u$ is necessary, otherwise, consumption streams constant where $u$ is concave and non constant elsewhere brings by means of Jensens inequality the quasiconcavity to default. The same kind of reasoning holds for the monotonicity and the upper semicontinuity.

Example 3.17. In the context of proposition 3.16, we compute $R$ in a specific case. As for the risk measure, we take

$$
\rho(c)=-\int_{0}^{1} u\left(\int_{0}^{t} e^{-\gamma(t-s)} d c_{s}\right) d t
$$

\footnotetext{
${ }^{6}$ All parameter functions are continuous, with $\theta(t, s)=0$ whenever $s \notin[0,1]$, positive elsewhere and $k_{1}, k_{2}$ are either constants or continuously differentiable. In [Hindy et al., 1992], they give weaker assumptions for those parameters. We report the reader to their article for their exact setting.
} 


\section{Illustrative Settings}

whereby $\gamma \geq 0$ and $u: \mathbb{R} \rightarrow \mathbb{R}$ is $C^{1}$, concave and increasing. The certainty equivalent is given by

$$
\begin{aligned}
& \alpha_{\min }(f, m)=\sup _{\left\{c \mid \int_{0}^{1} u\left(\int_{0}^{t} e^{-\gamma(t-s)} d c_{s}\right) d t \geq-m\right\}}-\int_{0}^{1} f(t) d c_{t} \\
& =\sup _{c \in \mathcal{C} \mathcal{S}} \int_{0}^{1}\left[-f(t) d c_{t}+\frac{1}{\beta}\left(u\left(\int_{0}^{t} e^{-\gamma(t-s)} d c_{s}\right)+m\right) d t\right],
\end{aligned}
$$

for some multiplier $\beta:=\beta(f, m)>0$. A consumption stream that optimized

$$
-f(t) d c_{t}+\frac{1}{\beta}\left(u\left(\int_{0}^{t} e^{-\gamma(t-s)} d c_{s}\right)+m\right)
$$

for any $t \in[0,1]$ is an optimizer for the computation of $\alpha_{\min }(f, m)$. The first order conditions $^{7}$ yields

$$
\int_{0}^{t} e^{-\gamma(t-s)} d \hat{c}_{s}=h\left(\frac{\beta f(t)}{1-e^{-\gamma t}}\right):=k(t), \quad 0<t<1,
$$

and $^{8} k(0)=0$. Derivation by $t$ yields a Voltera integral equation of the second kind

$$
d \hat{c}_{t}-\gamma \int_{0}^{t} e^{-\gamma(t-s)} d \hat{c}_{s} d t=k^{\prime}(t) d t, \quad 0<t<1,
$$

which solution is given by

$$
d \hat{c}_{t}=k^{\prime}(t) d t+\gamma k(t) d t
$$

Moreover, $\beta$ is uniquely determined by

$$
\int_{0}^{1} u\left(h\left(\frac{\beta f(t)}{1-e^{-\gamma t}}\right)\right) d t=-m .
$$

Plugging $\beta$ and $d \hat{c}$ into the expression of $\alpha_{\min }(f, m)$ and then inverting delivers $R$. In case where ${ }^{9} u(t)=-e^{-t}$, holds clearly $\alpha_{\min }(f, m)=0$ for $m \geq 0$, and elsewhere, direct

\footnotetext{
${ }^{7}$ Since the problem is smooth, and that the $C^{1}$ consumption streams approximate $\lambda^{l e b}$-almost surely any $c \in \mathcal{C S}$ we can write the first order conditions for differentiable consumption streams.

${ }^{8}$ Note that $h$ is uniformly bounded on $] 0,1\left[\right.$ as $f$ is Lipschitz with $f(0)=0$ and $\int_{0}^{t} e^{-\gamma(t-s)} d s$ is of order $t$ in a neighborhood of 0 .

${ }^{9}$ For other utility functions $u$, we refer to the techniques and forms of our robust representation of the certainty equivalent for random variables.
} 
computations yields:

$$
\begin{aligned}
\beta(f, m) & =m\left(\int_{0}^{1} \frac{f(t)}{1-e^{-\gamma t}} d t\right)^{-1} \\
k(t) & =\ln \left(\frac{1-e^{-\gamma t}}{m f(t)}\right)+\ln \left(\int_{0}^{1} \frac{f(s)}{1-e^{-\gamma s}} d s\right) \\
k^{\prime}(t) & =-\frac{f^{\prime}(t)}{f(t)}+\frac{\gamma e^{-\gamma t}}{1-e^{-\gamma t}} .
\end{aligned}
$$

Therefore

$$
\begin{aligned}
\alpha_{\min }(f, m) & =-\int_{0}^{1} f(t) d \hat{c}_{t} \\
& =-\int_{0}^{1} f(t) k^{\prime}(t) d t-\gamma \int_{0}^{1} f(t) k(t) d t \\
& =\gamma \ln (m) \int_{0}^{1} f(t) d t+g(f),
\end{aligned}
$$

whereby

$$
g(f)=\int_{0}^{1} d f_{t}-\gamma \int_{0}^{1} f(t)\left[\frac{e^{-\gamma t}}{1-e^{-\gamma t}}-\ln \left(\frac{f(t)}{1-e^{-\gamma t}}\right)+\ln \left(\int_{0}^{1} \frac{f(s)}{1-e^{-\gamma s}} d s\right)\right] d t .
$$

Inverting $\alpha_{\min }(f, m)$ yields:

$$
\rho(c)=\sup _{f \in \mathcal{C}_{+}^{\circ}} \exp \left(-\int_{0}^{1} \frac{f(s)}{\gamma} d c_{s}-\frac{g(f)}{\gamma}\right) .
$$

\subsection{Stochastic Kernels}

In Section 1.1, stochastic kernels were defined as measurable mappings $\tilde{X}: \Omega \rightarrow \mathcal{M}_{1, c}$, but they can equivalently ${ }^{10}$ be seen as functions $\tilde{X}: \Omega \times \mathscr{B} \rightarrow \mathbb{R}$ such that

(i) $\tilde{X}(\omega, \cdot) \in \mathcal{M}_{1, c}$ for $P$-almost all $\omega \in \Omega$,

(ii) $\tilde{X}(\cdot, A)$ is $\mathscr{F}$-measurable for any $A \in \mathscr{B}$,

$\overline{{ }^{10} \text { For the equivalence we refer to Lemma }} 1.40$ in [Kallenberg, 2002]. 


\section{Illustrative Settings}

whereby $(\Omega, \mathscr{F}, P)$ is a probability space, and $\mathscr{B}$ is the Borel $\sigma$-algebra on $\mathbb{R}$. We further denote by $\mathcal{S K}$ the space of those stochastic kernels which are uniformly bounded, that is, there exists some $c>0$ such that $\tilde{X}(\omega,[-c, c])=1$ for $P$-almost all $\omega \in \Omega$.

It is important to notice that those stochastic kernels which are scenario independent can be identified with $\mathcal{M}_{1, c}$. On the other hand, the bounded random variables $\mathbb{L}^{\infty}$ can be embedded into $\mathcal{S K}$ by way of the relation $\tilde{X}=\delta_{X}$ where $\tilde{X}(\omega, \cdot):=\delta_{X(\omega)}(\cdot)$ for any $X \in \mathbb{L}^{\infty}$.

As for the topology, we introduce the vector space $\mathcal{L C}$ of functions $H: \Omega \times \mathbb{R} \rightarrow \mathbb{R}$ such that $H(\omega, \cdot) \in C$ for $P$-almost all $\omega \in \Omega$ and $H(\cdot, x) \in \mathbb{L}^{1}$ for all $x \in \mathbb{R}$. We then consider on $\mathcal{S K}$ the weak topology $\sigma(\mathcal{S K}, \mathcal{L C})$ where the dual pairing is given by

$$
\langle H, \tilde{X}\rangle:=E\left[\int H(\cdot, s) \tilde{X}(\cdot, d s)\right] .
$$

This weak-topology can be seen as sort of mix between the $\sigma\left(\mathbb{L}^{\infty}, \mathbb{L}^{1}\right)$-topology on $\mathbb{L}^{\infty}$ and the weak ${ }^{*}$-topology on $\mathcal{M}_{1, c}$.

As for the preorder, we consider the $P$-almost sure $i$-th stochastic order for $i=1,2$ where $\tilde{X}$ is bigger that $\tilde{Y}$ if the lottery $\tilde{X}(\omega, \cdot)$ is bigger than $\tilde{Y}(\omega, \cdot)$ in the $i$-th stochastic order for $P$-almost all $\omega \in \Omega$. Since $\mathcal{S K}$ is neither closed nor open in the vector space of stochastic kernels $\tilde{X}: \Omega \rightarrow c a_{c}$, we can not apply Theorem 2.19. We therefore need some additional conditions to guarantee the existence of a robust representation. From the intrinsic mixed nature of stochastic kernels, we are interested in the interrelation between model risk and distributional risk. The adequate condition for a risk order $\succcurlyeq$ on $\mathcal{S K}$ to explicit such an interplay is the uniform preference monotonicity ${ }^{11}$ given by the relation

$$
\tilde{X}(\omega, \cdot) \succcurlyeq \tilde{Y}(\omega, \cdot) \text { for P-almost all } \omega \in \Omega \quad \Longrightarrow \quad \tilde{X} \succcurlyeq \tilde{Y} .
$$

Theorem 3.18. Let $\succcurlyeq$ be risk order on $\mathcal{S K}$, monotone with respect to the P-almost sure second stochastic order. Suppose furthermore that

(i) $\succcurlyeq$ is separable and lower semicontinuous,

(ii) $\delta_{s} \succ \delta_{t}$ for any two real $s, t$ with $s<t$,

(iii) $\succcurlyeq$ restricted on $\mathcal{M}_{1, c}$ is sensitive, that is

$$
\mu \succ \delta_{c} \text { for some } c \in \mathbb{R} \quad \Longrightarrow \quad \mu \succcurlyeq \delta_{c-\varepsilon} \text { for some } \varepsilon>0
$$

(iv) $\succcurlyeq$ satisfies the uniform preference monotonicity.

Then, $\succcurlyeq$ can be factorized in a model risk component and a distributional risk component.

${ }^{11} \mathrm{Be}$ aware that this monotonicity property is meant on the level of preferences in a $P$-almost sure sense and is not related to the monotonicity with respect to the preorder $\unrhd$. 
More precisely, any lower semicontinuous risk measure $\rho$ corresponding to $\succcurlyeq$ can be written as

$$
\rho=\Phi(-G)
$$

where $\Phi: \mathbb{L}^{\infty} \rightarrow \mathbb{R}$ is a lower semicontinuous risk measure, $G(\tilde{X})(\omega)=g(\tilde{X}(\omega, \cdot))$ for a lower semicontinuous risk measure $g: \mathcal{M}_{1, c} \rightarrow \mathbb{R}$ such that $g\left(\delta_{c}\right)=-c$ for any $c \in \mathbb{R}$. This decomposition has the robust representation

$$
\rho(\tilde{X})=\sup _{Q, u \in \mathcal{M}_{1}(P) \times \mathcal{K}^{2, \circ}} R\left(Q, E_{Q}\left[r\left(u,-\int u(s) \tilde{X}(\cdot, d s)\right)\right]\right), \quad \tilde{X} \in \mathcal{S} \mathcal{K},
$$

for a unique maximal risk function $R$ on probability measures and a unique maximal risk function $r$ on nondecreasing concave functions.

Conversely, such a representation implies the assumptions on $\succcurlyeq$.

The sensitivity assumption means that if a lotterie $\mu$ is strictly more risky than a Dirac measure, it remains then more risky even if we worsen the Dirac measure by shifting it by some small $\varepsilon$.

Proof. Let $\rho$ be a lower semicontinuous risk measure corresponding to $\succcurlyeq$. The restriction of $\succcurlyeq$ to $\mathcal{M}_{1, c}$ is $\sigma\left(\mathcal{M}_{1, c}, C\right)$-lower semicontinuous and by Theorem 3.10 there exists a lower semicontinuous risk measure $g: \mathcal{M}_{1, c} \rightarrow \mathbb{R}$ with robust representation

$$
g(\mu)=\sup _{u \in \mathcal{K}^{2, \circ}} r\left(u,-\int u d \mu\right)
$$

for a unique maximal risk function $r$. The function $h(c)=-g\left(\delta_{c}\right)$ for $c \in \mathbb{R}$ is increasing and upper semicontinuous. From the sensitivity and the strict monotonicity of $h$ holds $\operatorname{Im}(g)=\operatorname{Im}(h)$. Indeed, take some $\mu \in \mathcal{M}_{1, c}$ and suppose that $g(\mu) \notin \operatorname{Im}(h)$. Consider the smallest $t \in \mathbb{R}$ such that $\mu \succcurlyeq \delta_{t}$ which exists due to the lower semicontinuity. From the sensitivity, $\mu \sim \delta_{t}$ in contradiction to $g(\mu) \notin \operatorname{Im}(h)$ This shows that up to an increasing lower semicontinuous transformation $h^{-1}: \operatorname{Im}(g) \rightarrow \mathbb{R}$, we can suppose that $\operatorname{Im}(g)=\mathbb{R}$ and $g\left(\delta_{c}\right)=-c$. The lower semicontinuity implies moreover that $\omega \mapsto G(\tilde{X})(\omega):=g(\tilde{X}(\omega, \cdot))$ is measurable. The uniform preference monotonicity and the monotonicity implies that for any $k$ such that the support of $\tilde{X}$ lies uniformly in $[-k, k]$, it follows $k=g\left(\delta_{-k}\right) \geq G(\tilde{X}) \geq g\left(\delta_{k}\right)=-k$ showing that $G$ maps $\mathcal{S K}$ to $\mathbb{L}^{\infty}$. Moreover, the fact that ${ }^{12} G\left(\delta_{X}\right)=-X$ for any $X \in \mathbb{L}^{\infty}$ yields $\operatorname{Im}(G)=\mathbb{L}^{\infty}$.

We define now the functional $\Phi: G(\mathcal{S K})=\mathbb{L}^{\infty} \rightarrow \mathbb{R}$ as $\Phi(X)=\rho(\tilde{X})$ for $\tilde{X} \in$ $G^{-1}(-X)$. In order to be well defined, we have to show that for any $X \in \mathbb{L}^{\infty}$, the stochastic kernels in $G^{-1}(-X)$ are equivalent to each other. To do so, consider two stochastic kernels $\tilde{X}, \tilde{Y} \in \mathcal{S K}$ such that $G(\tilde{X})=G(\tilde{Y}) P$-almost surely. Then $g(\tilde{X}(\omega, \cdot))=g(\tilde{Y}(\omega, \cdot))$ for $P$-almost all $\omega \in \Omega$ and so $\tilde{X}(\omega, \cdot) \sim \tilde{Y}(\omega, \cdot)$. From the

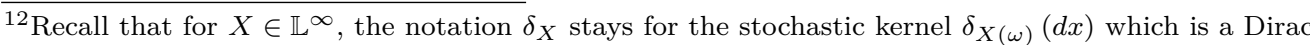
measure at $X(\omega)$ for any $\omega \in \Omega$.
} 


\section{Illustrative Settings}

uniform preference monotonicity, it follows that $\tilde{X} \sim \tilde{Y}$ and therefore, for any $X \in \mathbb{L}^{\infty}$, the elements of $G^{-1}(-X)$ are equivalents.

Concerning the monotonicity of $\Phi$, we use the same argumentation with inequality as for $X=-G(\tilde{X}) \leq-G(\tilde{Y})=Y$ it follows $\tilde{X} \succcurlyeq \tilde{Y}$ and therefore $\Phi(X)=\rho(\tilde{X}) \geq$ $\rho(\tilde{Y})=\Phi(Y)$.

As for the quasi-convexity, consider some $X, Y \in \mathbb{L}^{\infty}$ and set $\tilde{X}:=\delta_{-X}$ and $\tilde{Y}:=\delta_{-Y}$. Since $G\left(\delta_{X}\right)=-X$, holds $\Phi(X)=\rho(\tilde{X}), \Phi(Y)=\rho(\tilde{Y})$ and $\Phi(\lambda X+(1-\lambda) Y)=$ $\rho\left(\delta_{-\lambda X-(1-\lambda) Y}\right)$. The $P$-almost sure second stochastic order, yields $\delta_{-\lambda X-(1-\lambda) Y} \unrhd$ $\lambda \delta_{-X}+(1-\lambda) \delta_{-Y}$, since

$$
\begin{aligned}
\int u d \delta_{-\lambda X-(1-\lambda) Y}=u(-\lambda X-(1-\lambda) Y) \geq \lambda u & (-X)+(1-\lambda) u(-Y) \\
& =\lambda \int u d \delta_{-X}+(1-\lambda) \int u d \delta_{-Y},
\end{aligned}
$$

for any nondecreasing concave function $u$. Since $\rho$ is a risk measure it follows

$$
\begin{aligned}
\Phi(\lambda X+(1-\lambda) Y)=\rho\left(\delta_{-\lambda X-(1-\lambda) Y}\right) & \leq \rho\left(\lambda \delta_{-X}+(1-\lambda) \delta_{-Y}\right) \\
\leq \max & \left\{\rho\left(\delta_{-X}\right), \rho\left(\delta_{-Y}\right)\right\}=\max \{\Phi(X), \Phi(Y)\}
\end{aligned}
$$

showing that $\Phi$ is a risk measure.

Finally, from definition of $\rho$ and since it is lower semicontinuous, the functional $\Phi$ is $\sigma\left(\mathbb{L}^{\infty}, \mathbb{L}^{1}\right)$-lower semicontinuous and therefore, Theorem 3.3 yields

$$
\begin{aligned}
\rho(\tilde{X})=\Phi(-G(\tilde{X})) & =\sup _{Q \in \mathcal{M}_{1}(P)} R\left(Q, E_{Q}\left[\sup _{u \in \mathcal{K}^{2, \circ}} r\left(u,-\int u(x) \tilde{X}(\cdot, d x)\right)\right]\right) \\
& =\sup _{Q, u \in \mathcal{M}_{1}(P) \times \mathcal{K}^{2, \circ}} R\left(Q, E_{Q}\left[r\left(u,-\int u(x) \tilde{X}(\cdot, d x)\right)\right],\right.
\end{aligned}
$$

for some unique $R$ and $r$ respectively in the set of maximal risk functions for probability measures and of maximal risk functions for concave non decreasing functions.

As for the converse, it is plain to check that any representation of the form (3.30) and such that $\Phi(c)=-c$ for any $c \in \mathbb{R}$ satisfies the assumption on $\succcurlyeq$.

Under affine structure, we get ride of the lower semicontinuity assumption using our automatic continuity results to obtain a representation in the spirit of [Cerreia-Vioglio et al., 2008a].

Theorem 3.19. Let $\succcurlyeq$ be a risk order on $\mathcal{S K}$, monotone with respect to the P-almost sure $i$-th stochastic order for $i=1,2$. Suppose furthermore that

(i) $\succcurlyeq$ is separable,

(ii) $\delta_{s} \succ \delta_{t}$ for any two real $s, t$ with $s<t$, 
(iii) $\succcurlyeq$ satisfies the uniform preference monotonicity,

(iv) the restriction of $\succcurlyeq$ to $\mathcal{M}_{1, c}$ fulfills the Archimedian and independence axioms, and

(v) for any $\tilde{X}, \tilde{Y}, \tilde{Z} \in \mathcal{S K}$ with $\tilde{X} \succcurlyeq \tilde{Y}$, the set

$$
\{\lambda \in[0,1] \mid \tilde{X} \succcurlyeq \lambda \tilde{Y}+(1-\lambda) \tilde{Z}\}
$$

is closed in $[0,1]$.

Then there exists a lower semicontinuous risk measure $\rho: \mathcal{S K} \rightarrow \mathbb{R}$ corresponding to $\succcurlyeq$ such that

$$
\rho(\tilde{X})=\sup _{Q \in \mathcal{M}_{1, f}(P)} R\left(Q, E_{Q}\left[-\int u(s) \tilde{X}(\cdot, d s)\right]\right), \quad \tilde{X} \in \mathcal{S K},
$$

for some $u \in \mathcal{K}^{i, \circ}$ such that $\left.u: \mathbb{R} \rightarrow\right] a, b[$ with $-\infty \leq a<b \leq+\infty$ and a maximal risk function $\left.R: \mathcal{M}_{1, f} \times\right] a, b[\rightarrow[-\infty,+\infty]$ as stated in Theorem 3.3 such that

$$
\Phi(X):=\sup _{Q \in \mathcal{M}_{1, f}(P)} R\left(Q, E_{Q}[-X]\right), \quad X \in \mathbb{L}^{\infty},
$$

satisfies $\Phi(c)=-c$ for all $c \in \mathbb{R}$.

If moreover $\Phi$ has the Fatou property, the supremum in (3.32) and (3.33) can be reduced to the set $\mathcal{M}_{1}(P)$.

Proof. The conditions $(i i),(i v)$ and $(v)$ ensures that the restriction of $\succcurlyeq$ to $\mathcal{M}_{1, c}$ is a risk order fulfilling the requirement of Theorem 2.24. There exists then an increasing ${ }^{13}$ upper semicontinuous function $u: \mathbb{R} \rightarrow] a, b[$, where $-\infty \leq a<b \leq+\infty$, such that $\mu \rightarrow-\int u d \mu$ is a risk order corresponding to $\succcurlyeq$ restricted to $\mathcal{M}_{1, c}$. We can suppose up to a translation that $a<0<b$. Define now $F(\tilde{X})(\omega):=-\int u(x) \tilde{X}(\omega, d x)$ for any $\tilde{X} \in \mathcal{S K}$. Since,

$$
F(\lambda \tilde{X}+(1-\lambda) \tilde{Y})=\lambda F(\tilde{X})+(1-\lambda) F(\tilde{Y})
$$

for any $\tilde{X}, \tilde{Y} \in \mathcal{S K}$ and $\lambda \in] 0,1[$, it follows that $F(\mathcal{S K})=(-B,-A)$ for the notations of Theorem 3.3.

By the separability condition $(i)$, consider a risk measure $\rho: \mathcal{S K} \rightarrow[-\infty,+\infty]$ corresponding to $\succcurlyeq$ such that, via an increasing transformation, $\rho(\mu)=-\int u d \mu$. By the monotonicity assumption, $\rho$ takes values in $]-b,-a[$.

We now define $\Phi:(A, B) \rightarrow \mathbb{R}$ by $\Phi(X)=\rho(\tilde{X})$ for $\tilde{X} \in F^{-1}(-X)$. The uniform preference monotonicity can be applied with the same argumentation as in the proof of Theorem 3.18 to ensure that $\Phi$ is well defined and monotone but the argumentation is no longer valid for the quasi-convexity as we can not apply the fact that $F\left(\delta_{X}\right)=-X$.

${ }^{13}$ Because of condition $(i i)$. 


\section{Illustrative Settings}

However, the affinity of $F$ implies the quasiconvexity of $\Phi$ by means of the quasiconvexity of $\rho$ and relation (3.34). Therefore, $\Phi$ is a risk measure with image $]-b,-a[$. Applying now Theorem 3.3 to $\Phi$ ends the proof.

The previous theorem generalizes the representation in [Maccheroni et al., 2006b, Theorem 3] in the sense that we only require a directional lower semicontinuity in (3.31) rather than a directional continuity. This implies in particular, that we do not necessarily have a certainty equivalent. Moreover, we use our automatic continuity result to get a von Neumann and Morgenstern representation on $\mathcal{M}_{1, c}$. Finally, we make use of the Fatou property to restrict the representation to the set of probability measures.

The case that $\Phi$ in Theorem 3.19 is a cash additive risk measure corresponds to the variational preferences as introduced by Maccheroni et al. [2006a]. This is the subject of the following proposition.

Proposition 3.20. Let $\succcurlyeq$ be a risk order satisfying the same assumptions as in Theorem 3.19. Suppose in addition that $\succcurlyeq$ satisfies

(vi) the weak certainty condition, that is, for any $\tilde{X}, \tilde{Y} \in \mathcal{S K}$ and any $c_{0} \in \mathbb{R}$ holds

$$
\begin{aligned}
\lambda \tilde{X}+(1-\lambda) \delta_{c_{0}} & \sim \lambda \tilde{Y}+(1-\lambda) \delta_{c_{0}} \\
\Longrightarrow & \lambda \tilde{X}+(1-\lambda) \delta_{c} \sim \lambda \tilde{Y}+(1-\lambda) \delta_{c}, \quad \text { for any } c \in \mathbb{R} .
\end{aligned}
$$

Then, there exists a lower semicontinuous risk measure $\rho$ related to $\succcurlyeq$ with robust representation

$$
\rho(\tilde{X})=\sup _{Q \in M_{1, f}(P)}\left\{E_{Q}\left[-\int u(x) \tilde{X}(\cdot, d x)\right]-\alpha_{\min }(Q)\right\}, \quad \tilde{X} \in \mathcal{S} \mathcal{K} .
$$

where $u: \mathbb{R} \rightarrow \mathbb{R}$ is continuous increasing $\alpha_{\min }: \mathcal{M}_{1, f}(P) \rightarrow[0, \infty]$ is a minimal penalty function.

Proof. Since the same assumptions than in Theorem 3.19 hold, we use here the same notations as in its proof. We will show that under the assumption of weak uncertainty we can extend $\Phi$ from $(A, B)$ into a cash additive risk measure on $\mathbb{L}^{\infty}$. As we will see, the maximal extension of Theorem 3.3 is not the right one since it is not cash additive if $(A, B) \neq \mathbb{L}^{\infty}$.

In a first step, we show that

$$
\Phi(X+n)=\Phi(X)-n,
$$

for any $X \in(A, B)$ and $n \in \mathbb{R}$ such that $X+n \in(A, B)$. To this end, consider $X, Y \in(A, B)$ with $\Phi(X)=\Phi(Y)$ and notice that there exists $\lambda \in] 0,1[$ such that $X / \lambda, Y / \lambda \in(A, B)$. Then, $\lambda F^{-1}(X / \lambda) \sim \lambda F^{-1}(Y / \lambda)$ and (3.35) yields

$$
\lambda F^{-1}\left(\frac{X}{\lambda}\right)+(1-\lambda) \delta_{c} \sim \lambda F^{-1}\left(\frac{Y}{\lambda}\right)+(1-\lambda) \delta_{c} \quad \text { for all } c \in \mathbb{R} .
$$


This shows

$$
\Phi(X+(1-\lambda) u(c))=\Phi(Y+(1-\lambda) u(c)) \quad \text { for all } c \in \mathbb{R}
$$

Take now $X \in(A, B)$, and $\lambda \in] 0,1[$ such that $X / \lambda,-\Phi(X) / \lambda \in(A, B)$. Since $\Phi(n)=$ $-n$, setting $Y=-\Phi(X)$ and $c=u^{-1}(n /(1-\lambda))$, then for any $\left.n \in\right](1-\lambda) a,(1-\lambda) b[$ holds (3.38), and therefore

$$
\Phi(X+n)=\Phi\left(-\Phi(X)+(1-\lambda) u\left(u^{-1}(n)\right)\right)=\Phi(X)-n
$$

This means in particular the Gateau differential in direction of 1 of $\Phi$ at any point in $(A, B)$ is equal to -1 , and therefore:

$$
\Phi(X+m)=\Phi(X)+\int_{0}^{m} D_{1} \Phi(Z)_{\mid Z=X+s} d s=\Phi(X)-\int_{0}^{m} d s=\Phi(X)-m
$$

for any $m \in \mathbb{R}$ such that $X+m \in(A, B)$.

In a second step, we extend $\Phi$ on $\mathbb{L}^{\infty}$ by

$$
\hat{\Phi}(X)=\inf \{\Phi(Y)-m \mid X \geq Y+m \text { with }(Y, m) \in(A, B) \times \mathbb{R}\}, \quad X \in \mathbb{L}^{\infty} .
$$

It is clear that $\hat{\Phi}$ is monotone, quasiconvex and cash additive. Let us show that $\hat{\Phi}$ coincides with $\Phi$ on $(A, B)$. To do so, consider some $X \in(A, B)$. It is straightforward to check that those pairs $Y+m$ such that $Y+m \notin(A, B)$ are not optimal. This together with the cash additivity and the monotonicity of $\Phi$ on $(A, B)$ yields

$$
\begin{array}{r}
\hat{\Phi}(X)=\inf \{\Phi(Y)-m \mid X \geq Y+m,(Y, m) \in(A, B) \times \mathbb{R} \text { and } Y+m \in(A, B)\} \\
=\inf \left\{\Phi\left(Y^{\prime}\right) \mid X \geq Y \text { with } Y^{\prime} \in(A, B)\right\}=\Phi(X) .
\end{array}
$$

Hence, $\hat{\Phi}$ is a cash additive risk measure which extends $\Phi$. By Theorem 3.3 and Proposition $2.17, \hat{\Phi}$ has a robust representation of the form

$$
\hat{\Phi}(X)=\sup _{Q \in \mathcal{M}_{1, f}(P)}\left\{E_{Q}[-X]-\alpha_{\min }(Q)\right\}, \quad X \in \mathbb{L}^{\infty}
$$

for a minimal penalty function $\alpha_{\min }$.

Example 3.21. The following example illustrates how to assess both model risk and distributional risk in a financial context with stochastic kernels. A financial institution delivers long term insurance products which default distribution depends on the temperature. In the IPCC [2007] report, the future temperature distribution is computed according to physical models for a given set of parameters. However, the value of these parameters depend on different scenarios as pictured in Figure 3.1. These scenarios depict among others the possible evolutions of the population, the economy, the technology, the energy, or the agriculture. The four main scenarios described in [IPCC, 


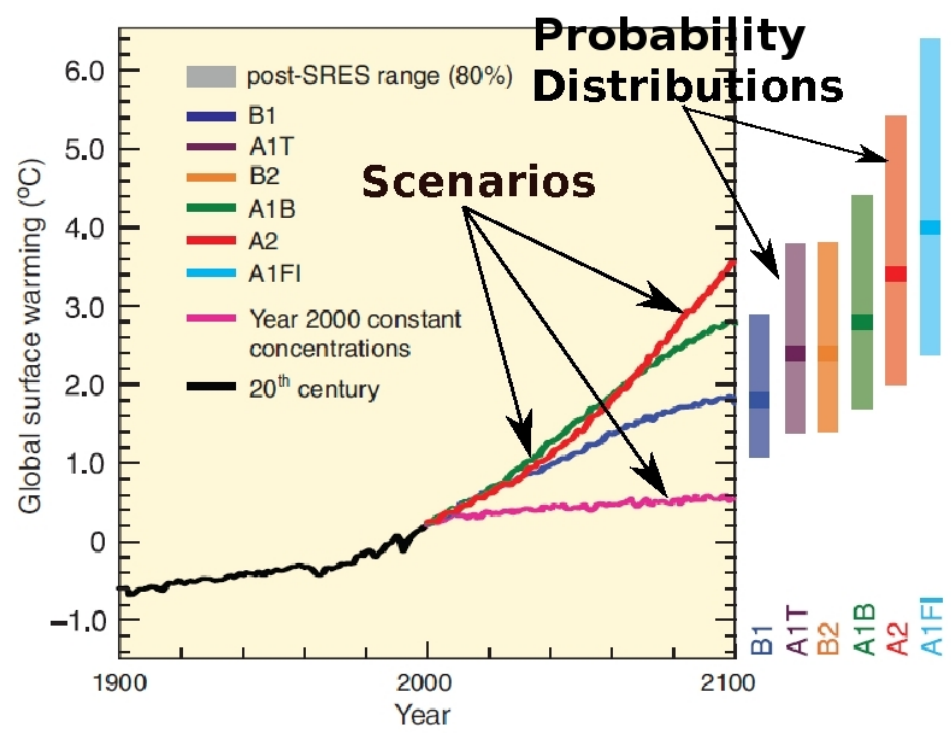

Figure 3.1.: Scenarios for green house gas emissions from 2000 to 2100 (in the absence of additional climate policies) and projections of surface temperatures. Sources [IPCC, 2007].

2000] are

$\omega_{1}=$ scenario A1: a future world of very rapid economic growth, global population that peaks in mid-century and declines thereafter, and rapid introduction of new and more efficient technologies.

$\omega_{2}=$ scenario A2: a very heterogeneous world with continuously increasing global population and regionally oriented economic growth that is more fragmented and slower than in other storylines.

$\omega_{3}=$ scenario B1: a convergent world with the same global population as in the A1 storyline but with rapid changes in economic structures toward a service and information economy, with reductions in material intensity, and the introduction of clean and resource-efficient technologies.

$\omega_{4}=$ scenario B2: a world in which the emphasis is on local solutions to economic, social, and environmental sustainability, with continuously increasing population (lower than A2) and intermediate economic development.

The scenario dependent default distribution of an insurance contract is then given by

$$
\tilde{X}(\omega, d x)=\mu_{1}(d x) 1_{\omega_{1}}(\omega)+\mu_{2}(d x) 1_{\omega_{2}}(\omega)+\mu_{3}(d x) 1_{\omega_{3}}(\omega)+\mu_{4}(d x) 1_{\omega_{4}}(\omega),
$$

where $\mu_{i} \in \mathcal{M}_{1, c}$ corresponds to the default distribution related to the temperature distribution estimation in the scenario $\omega_{i}$ for $i=1, \ldots, 4$. 
For a given distribution, the financial institution assesses the risk by means of the "Value at Risk", see Example 3.13. However, there is still uncertainty as for the probability occurrence of either one scenario or the other. To do so, it first computes the Value at Risk of the distribution in each scenario. It average then the result under any possible probability measure $Q=\left(q_{1}, q_{2}, q_{3}, q_{4}\right)$. It finally penalizes the result for those probability $Q$ which are distant from a likeliest probability $P$ according to some penalty function $\alpha$ given by a $\varphi$-divergence, see Example 3.6. This results in the following risk measure

$$
\begin{aligned}
\rho(\tilde{X}) & =\sup _{Q=\left(q_{1}, q_{2}, q_{3}, q_{4}\right)}\left\{E_{Q}\left[\sum_{i=1}^{4} V @ R_{q}\left(\mu_{i}\right) 1_{\omega_{i}}\right]-\alpha(Q \mid P)\right\} \\
& =\sup _{Q=\left(q_{1}, q_{2}, q_{3}, q_{4}\right)}\left\{\sum_{i=1}^{4}\left[V @ R_{q}\left(\mu_{i}\right) \frac{q_{i}}{p_{i}}-\varphi\left(\frac{q_{i}}{p_{i}}\right)\right] p_{i}\right\},
\end{aligned}
$$

which takes into account both distributional risk and model risk. 



\section{Part II.}

\section{Conditional Preferences}





\section{Conditional Preferences}

\subsection{Axiomatic and First Results}

In the general framework of part II, the risky elements will be contingent on different scenarios, as in the setting of ANSCOMBE AND AUMAnN [1963]. Mathematically, they will be described by measurable functions $\tilde{X}: \Omega \rightarrow \mathcal{X}$, where $(\Omega, \mathscr{F})$ is a measurable space of scenarios, and $(\mathcal{X}, \mathscr{X})$ is a measurable space of positions, whereby $\mathcal{X}$ is a convex set. A constant position is a position which is state independent, that is, $x:=x 1_{\Omega}$ for $x \in \mathcal{X}$. Constant positions will usually be denoted with small capital letters $x, y, \ldots$ as the element of $\mathcal{X}$. The conditional preferences will be specified on a convex subset, generically denoted by $\tilde{\mathcal{X}}$, of those state dependent positions. We suppose that this convex set contains the constants, that is, $\mathcal{X} \subset \tilde{\mathcal{X}}$. Standard examples for $\mathcal{X}$ include

- $\mathcal{X}=\mathbb{R}$. In this case, some possible sets are the vector spaces $\tilde{\mathcal{X}}=\mathbb{L}^{p}(\Omega, \mathscr{F}, P)$, for $p \in[1,+\infty]$.

- $\mathcal{X}=\mathcal{C} \mathcal{S}_{+}$the set of consumption streams introduced in Section 1.1. In this case, $\tilde{\mathcal{X}}$ could be the set of consumption plans contingent on scenarios. They were used for instance by BANK AND RIEDEL [2001].

- $\mathcal{X}=\mathcal{M}_{1, c}$, the set of probability distributions on the real line with bounded support. Here, $\tilde{\mathcal{X}}$ corresponds to the set of stochastic kernels on the real line, introduced in Section 1.1.

The conditionality of the preference order will refer to a fixed sub-sigma-algebra $\mathscr{G} \subset \mathscr{F}$ which represents the information needed to express preferences. We fix a reference measure $P$ on the smaller measurable set $(\Omega, \mathscr{G})$ in order to specify the null sets of $\mathscr{G}$. As mentioned in the introduction, in previous work on conditional preferences the preference structure was given by a possibly uncountable family of standard preference relations indexed by the elements of $\mathscr{G}$; cf. [Skiadas, 1997a,b]. Here we consider instead the conditional preference structure with respect to $\mathscr{G}$ as a single binary relation $\succcurlyeq$ defined on $\tilde{\mathcal{X}}$. In our context we cannot expect completeness of the relation. Our binary relation will only be a preorder, sometimes also called quasiorder, that is $\succcurlyeq$ fulfills the following axiom:

A-I Preorder: A binary relation $\succcurlyeq$ on $\tilde{\mathcal{X}}$ is a preorder if the following properties hold:

(a) Reflexivity: $\tilde{X} \succcurlyeq \tilde{X}$ for any $\tilde{X} \in \tilde{X}$.

(b) Transitivity: If $\tilde{X} \succcurlyeq \tilde{Y}$ and $\tilde{Y} \succcurlyeq \tilde{Z}$ then $\tilde{X} \succcurlyeq \tilde{Z}$. 


\section{Conditional Preferences}

Remark 4.1. Any preorder induces an equivalence (indifference) relation $\sim$ given by

$$
\tilde{X} \sim \tilde{Y} \quad \Longleftrightarrow \quad \tilde{X} \preccurlyeq \tilde{Y} \text { and } \tilde{Y} \succcurlyeq \tilde{X}
$$

and a strict preorder $\succ$ defined by

$$
\tilde{X} \succ \tilde{Y} \Longleftrightarrow \tilde{X} \succcurlyeq \tilde{Y} \text { and } \tilde{X} \npreceq \tilde{Y} \quad \Longleftrightarrow \quad \tilde{X} \succcurlyeq \tilde{Y} \text { and } \tilde{X} \nsim \tilde{Y}
$$

We now describe how our preorder is related to the given sub-sigma-algebra $\mathscr{G}$, that is, which consistency conditions it has to fulfill in order to be a $\mathscr{G}$-conditional preference relation. Beforehand, to describe the preference between two elements on an event $A \in \mathscr{G}$, we set these two elements as constant on the complement of this event. To this aim, we introduce a reference constant $x_{0} \in \mathcal{X}$. For $\tilde{X} \in \tilde{\mathcal{X}}$ and $A \in \mathscr{G}$, we use the notation $\tilde{X} 1_{A}$ for the position $\tilde{X} 1_{A}+x_{0} 1_{A^{c}}$ equal to $\tilde{X}$ on $A$ and reduced to the constant $x_{0}$ outside $A$.

A-II $\mathscr{G}$-Conditional Consistency: A binary relation $\succcurlyeq$ on $\tilde{X}$ is consistent with respect to $\mathscr{G}$ if for any $\tilde{X}, \tilde{Y} \in \tilde{\mathcal{X}}$ the following properties hold:

(a) Negligibility: For any $A, B \in \mathscr{G}$ with $P[A]=P[B]=1$ holds

$$
\tilde{X} \succcurlyeq \tilde{Y} \quad \Longleftrightarrow \quad \tilde{X} 1_{A} \succcurlyeq \tilde{Y} 1_{B}
$$

(b) Local completeness: There exists a set $A \in \mathscr{G}$ of strict positive probability such that

$$
\tilde{X} 1_{A} \succcurlyeq \tilde{Y} 1_{A} \quad \text { or } \quad \tilde{X} 1_{A} \preccurlyeq \tilde{Y} 1_{A}
$$

(c) Union consistency: For any countable family $\left(A_{n}\right)_{n \in \mathbb{N}}$ in $\mathscr{G}$,

$$
\forall n \in \mathbb{N}, \tilde{X} 1_{A_{n}} \succcurlyeq \tilde{Y} 1_{A_{n}} \Longrightarrow \tilde{X} 1_{\left\{\bigcup_{n \in \mathbb{N}} A_{n}\right\}} \succcurlyeq \tilde{Y} 1_{\left\{\bigcup_{n \in \mathbb{N}} A_{n}\right\}}
$$

(d) Reducibility: For $A, B \in \mathscr{G}$ with $A \supseteq B$,

$$
\tilde{X} 1_{A} \succcurlyeq \tilde{Y} 1_{A} \quad \Longrightarrow \quad \tilde{X} 1_{B} \succcurlyeq \tilde{Y} 1_{B}
$$

Definition 4.2. A $\mathscr{G}$-conditional preference relation on $\tilde{\mathcal{X}}$ is a binary relation satisfying axioms A-I and A-II. In that case we use the notation $\succcurlyeq^{\mathscr{G}}$. Furthermore, for $A \in \mathscr{G}$ and $\tilde{X}, \tilde{Y} \in \tilde{\mathcal{X}}$, we write $\tilde{X} \succcurlyeq_{A}^{\mathscr{G}} \tilde{Y}$ for $\tilde{X} 1_{A} \succcurlyeq^{\mathscr{G}} \tilde{Y} 1_{A}$.

Remark 4.3. For a $\mathscr{G}$-conditional relation $\succcurlyeq^{\mathscr{G}}$, the negligibility assumption together with the reducibility assumption yields immediately

$$
\tilde{X} \succcurlyeq^{\mathscr{G}} \tilde{Y} \quad \Longleftrightarrow \quad \tilde{X} \succcurlyeq_{A}^{\mathscr{G}} \tilde{Y} \quad \text { for any } A \in \mathscr{G}
$$


Intuitively, the negligibility assumption guarantees that conditional preference relations are relevant only on those events of $\mathscr{G}$ which have a positive probability under $P$. As for reducibility, it translates the fact that if $\tilde{X}$ is preferred to $\tilde{Y}$ over some event of positive probability, then, whenever this preference is considered on some sub-event with strict positive probability, $\tilde{X}$ remains preferred to $\tilde{Y}$. This insures in particular that events over which $\tilde{X}$ is strictly preferred to $\tilde{Y}$ and events over which $\tilde{Y}$ is strictly preferred to $\tilde{X}$ have an intersection of zero measure. The interpretation of the union consistency is straightforward.

The local completeness property of the $\mathscr{G}$-consistency axiom is the crucial point distinguishing a conditional preference from its unconditional counterpart: Instead of global completeness we require only that $\tilde{X}$ and $\tilde{Y}$ can be compared at least over some event of positive probability. Suppose for instance, you had to compare the two different opportunities of spending next month one day on the seacoast or attending an exposition in some museum. Unless you have a very strong affinity or aversion for one over the other, you may be unable to achieve a honest choice between them without additional information or even to say that you are indifferent between both of them. But conditioned on the additional information that this day will be a very sunny one, you might prefer the seacoast over the museum. Or similarly, suppose you are thinking about investing wealth in treasury bonds, but you are not really able - even do not want - to choose between dollar and euro on your actual knowledge. However, a decision for one or the other could be achieved under the additional information given by the central banks next week concerning their respective decision of maintaining or changing their interest rates.

A $\mathscr{G}$-conditional preference order has the following properties:

Proposition 4.4. For a $\mathscr{G}$-conditional preference relation $\succcurlyeq^{\mathscr{G}}$, the properties of negligibility, union consistency and reducibility also hold for the induced equivalence relation $\sim^{\mathscr{G}}$. The induced strict preference relation $\succ^{\mathscr{G}}$ inherits the properties of negligibility and union consistency.

Moreover, for any countable family $\left(A_{n}\right)_{n \in \mathbb{N}}$ of elements of $\mathscr{G}$,

$$
\tilde{X} \succ_{\left\{\bigcup_{n \in \mathbb{N}}^{\mathscr{G}} A_{n}\right\}} \tilde{Y} \Longrightarrow \tilde{X} \succ_{A_{n_{0}}}^{\mathscr{G}} \tilde{Y} \quad \text { for at least one } n_{0} \in \mathbb{N} \text {. }
$$

For notational convenience, the reference to the $\sigma$-algebra $\mathscr{G}$ will be dropped in proofs if there is no possible misunderstanding.

Proof. Negligibility for $\sim$ and $\succ$ as well as union consistency and reducibility for $\sim$ immediately follow from their definition.

Let us now show union consistency for $\succ$. Suppose that for any $n \in \mathbb{N}, \tilde{X} \succ_{A_{n}} \tilde{Y}$. The union consistency for $\succcurlyeq$ yields $\tilde{X} \succcurlyeq_{\left\{\bigcup A_{n}\right\}} \tilde{Y}$. If $\tilde{X} \sim_{\left\{\bigcup A_{n}\right\}} \tilde{Y}$ were true, the reducibility property for $\sim$ would imply $\tilde{X} \sim_{A_{n}} \tilde{Y}$ for any $n \in \mathbb{N}$, in contradiction to $\tilde{X} \succ_{A_{n}} \tilde{Y}$. This shows the union consistency for $\succ$.

Finally, we show relation (4.1). Suppose that $\tilde{X} \succ_{\left\{\cup A_{n}\right\}} \tilde{Y}$. From the reducibility assumption for $\succcurlyeq$ follows $\tilde{X} \succcurlyeq_{A_{n}} \tilde{Y}$ for any $n \in \mathbb{N}$. If $\tilde{X} \sim_{A_{n}} \tilde{Y}$ holds for any $n \in \mathbb{N}$, 


\section{Conditional Preferences}

union consistency for $\sim$ yields $\tilde{X} \sim_{\left\{\cup A_{n}\right\}} \tilde{Y}$ which contradicts our assumption $\tilde{X} \succ_{\left\{\cup A_{n}\right\}}$ $\tilde{Y}$. Hence, there exists $n_{0} \in \mathbb{N}$ such that $\tilde{X} \succ_{A_{n_{0}}} \tilde{Y}$.

Even though the assumption of local completeness looks very weak, it allows us to compare any two stochastic kernels in the sense specified by the following lemma:

Lemma 4.5. Consider a $\mathscr{G}$-conditional preference relation $\succcurlyeq^{\mathscr{G}}$. Then for each $\tilde{X}, \tilde{Y} \in$ $\tilde{\mathcal{X}}$ there exists a partition $A, B, C \in \mathscr{G}$ of $\Omega$ such that

$$
\tilde{X} \succ_{A}^{\mathscr{G}} \tilde{Y} \quad, \quad \tilde{X} \prec_{B}^{\mathscr{G}} \tilde{Y} \quad \text { and } \quad \tilde{X} \sim_{C}^{\mathscr{G}} \tilde{Y}
$$

Moreover, this partition can be chosen maximally, i.e., $P\left[C^{\prime} \backslash C\right]=0$ for any $C^{\prime} \supset C$ with $\tilde{X} \sim_{C^{\prime}} \tilde{Y}$; equivalently, $\tilde{X} \succ_{A^{\prime}}^{\mathscr{Y}} \tilde{Y}$ resp $\tilde{X} \prec_{B^{\prime}}^{\mathscr{G}} \tilde{Y}$ for any $A^{\prime} \subset A$ resp $B^{\prime} \subset B$.

Proof. On $\mathscr{G}$, we consider the preorder $\sqsubseteq$ defined for $A, B \in \mathscr{G}$ as follow:

$$
A \sqsubseteq B \quad \Longleftrightarrow \quad P[A \backslash B]=0
$$

This preorder induces an equivalent relation denoted by $\cong$ identifying sets which are equal almost surely. Passing to equivalence classes, we obtain the set $\mathscr{G} / \cong$ which is partially ordered under $\sqsubseteq$. Due to negligibility, our conditional preference relation $\succcurlyeq$ can be factorised as well. We can then define the following classes of sets in $\mathscr{G} / \cong$ :

$$
\begin{gathered}
\mathcal{A}=\left\{A \in \mathscr{G}|\cong| \tilde{X} \succ_{A^{\prime}} \tilde{Y} \text { for any } \mathscr{G} \ni A^{\prime} \sqsubseteq A\right\}, \\
\mathcal{B}=\left\{B \in \mathscr{G}|\cong| \tilde{X} \succ_{B^{\prime}} \tilde{Y} \text { for any } \mathscr{G} \ni B^{\prime} \sqsubseteq B\right\} \quad \text { and } \mathcal{C}=\left\{C \in \mathscr{G}|\cong| \tilde{X} \sim_{C} \tilde{Y}\right\},
\end{gathered}
$$

partially ordered for $\sqsubseteq$.

We show first that each class admits a maximal element. To do so, consider a complete ordered chain $\left(A_{i}\right)_{i \in I}$ in $\mathcal{A}$. We set $p=\sup _{i \in I} P\left[A_{i}\right]$ and pick an increasing sequence of sets $\left(A_{n}\right)_{n}$ within this chain such that $P\left[A_{n}\right] \geq p-1 / n$. Setting $\bar{A}=\bigcup_{n \in \mathbb{N}} A_{n}$, the $\sigma$ additivity of $P$ yields $P[\bar{A}]=p$. The union consistency property of $\succ$ from Proposition 4.4 yields $\tilde{X} \succ_{A} \tilde{Y}$. Moreover, for any $A^{\prime} \sqsubseteq A$, we have $\tilde{X} \succcurlyeq_{A^{\prime}} \tilde{Y}$, therefore, if $\tilde{X} \sim_{A^{\prime}} \tilde{Y}$ were true, then for any $n \in \mathbb{N}, A_{n}^{\prime}=A_{n} \cap A \sqsubseteq A_{n}$ would imply $\tilde{X} \sim_{A_{n}^{\prime}} \tilde{Y}$ in contradiction with $A_{n} \in \mathcal{A}$ for any $n$. Hence $A \in \mathcal{A}$. Take now $A_{i}$ in this chain such that $\bar{A} \sqsubseteq A_{i}$. Then $P\left[A_{i} \backslash \bar{A}\right] \leq P\left[A_{i}\right]-p \leq 0$ and thus $A_{i}=\bar{A}$ in $\mathscr{G} / \cong$ proving that $\bar{A}$ is an upper bound for the chain. Having a partial order, we apply Zorn's lemma to obtain the existence of a maximal element $\tilde{A} \in \mathcal{A}$. With the same argumentation, there exists also two maximal elements $\tilde{B}$ and $\tilde{C}$ of $\mathcal{B}$ and $\mathcal{C}$ respectively.

The definition of $\mathcal{A}, \mathcal{B}$ and $\mathcal{C}$ implies immediately that $\tilde{A} \cap \tilde{B}=\tilde{A} \cap \tilde{C}=\tilde{B} \cap \tilde{C}=\emptyset$.

We are left to show that these three sets build a partition of $\Omega P$-almost surely. Suppose that $D=(\tilde{A} \cup \tilde{B} \cup \tilde{C})^{c}$ has a positive probability. The local completeness assumption yields the existence of $E \subset D$ with positive measure such that $\tilde{X} \succ_{C} \tilde{Y}$ or $\tilde{X} \prec_{C} \tilde{Y}$ or $\tilde{X} \sim_{C} \tilde{Y}$. In each case, $C$ belongs to one of the $\mathcal{A}, \mathcal{B}$ or $\mathcal{C}$, contradicting the maximality of one of $\tilde{A}, \tilde{B}$ or $\tilde{C}$. 
For unconditional preferences, a numerical representation maps the preference order into $\mathbb{R}$. In our case, a $\mathscr{G}$-preference relation will be represented by a numerical representation in the following sense:

Definition 4.6. A numerical representation for a $\mathscr{G}$-conditional preference relation $\succcurlyeq^{\mathscr{G}}$ is a functional $\tilde{U}: \tilde{\mathcal{X}} \rightarrow \mathbb{L}^{\infty}(\Omega, \mathscr{G}, P)$ such that

$$
\tilde{U}(\tilde{X}) \geq \tilde{U}(\tilde{Y}) \quad P \text {-almost surely } \quad \Longleftrightarrow \quad \tilde{X} \succcurlyeq^{\mathscr{G}} \tilde{Y}
$$

and having the property

$$
\tilde{U}\left(\tilde{X} 1_{A}\right)=\tilde{U}(\tilde{X}) 1_{A}+\tilde{U}\left(\tilde{x}_{0}\right) 1_{A^{c}}
$$

for any $\tilde{X} \in \tilde{\mathcal{X}}$ and $A \in \mathscr{G}$.

Any functional $\tilde{U}: \tilde{\mathcal{X}} \rightarrow \mathbb{L}^{\infty}(\Omega, \mathscr{G}, P)$ satisfying property (4.4) clearly defines a $\mathscr{G}$ preference relation $\succcurlyeq^{\mathscr{G}}$ via relation (4.3). The induced equivalence relation $\tilde{X} \sim^{\mathscr{G}} \tilde{Y}$ holds if and only if $\tilde{U}(\tilde{X})=\tilde{U}(\tilde{Y}) P$-almost surely. The induced strict preference relation $\tilde{X} \succ^{\mathscr{G}} \tilde{Y}$ is valid if and only if $\tilde{U}(\tilde{X}) \geq \tilde{U}(\tilde{Y}) P$-almost surely and $P[\tilde{U}(\tilde{X})>\tilde{U}(\tilde{Y})]>0$. This shows that the strict preference relation should not be understood as " $\tilde{X}$ is strictly better than $\tilde{Y}$ almost surely": Strict inequality holds only on a set of positive probability. Moreover, for any $\tilde{X}, \tilde{Y} \in \tilde{\mathcal{X}}$, the sets

$$
A=\{\tilde{U}(\tilde{X})>\tilde{U}(\tilde{Y})\}, B=\{\tilde{U}(\tilde{X})<\tilde{U}(\tilde{Y})\} \text {, and } C=\{\tilde{U}(\tilde{X})=\tilde{U}(\tilde{Y})\}
$$

constitute a maximal partition for the induced $\mathscr{G}$-conditional preference relation. If $0<P[C]<1, P[A]>0$, and $P[B]>0$, then the partitions

$$
\left\{A^{\prime}=A \cup C, B, \emptyset\right\} \text { and }\left\{A, B^{\prime}=B \cup C, \emptyset\right\},
$$

also satisfy relation (4.2), but they are not maximal.

Note that our preference relation $\succcurlyeq^{\mathscr{G}}$ can be recovered in a very natural way from its numerical representation, in contrast to the formalism in [Skiadas, 1997a,b], where the reconstruction of the family of preferences from a numerical representation is not as clear.

Conversely, and this justifies our definition of a numerical representation, a conditional separability condition together with Lemma 4.5 allows the construction of a numerical representation from the conditional preference order.

A $\mathscr{G}$-conditional preference relation is said to be conditionally separable if there exists a countable set $\tilde{\mathcal{Z}} \subset \tilde{\mathcal{X}}$ such that whenever $\tilde{X} \succ^{\mathscr{G}} \tilde{Y}$, then for the maximal set $A \in \mathscr{G}$ provided by Lemma 4.5 for which $\tilde{X} \succ_{A}^{\mathscr{G}} \tilde{Y}$, holds

$$
\tilde{X} \succcurlyeq_{A}^{\mathscr{G}} \sum_{n \in \mathbb{N}} \tilde{Z}_{n} 1_{A_{n}} \succcurlyeq_{A}^{\mathscr{G}} \tilde{Y}
$$

for some $\mathscr{G}$-measurable partition $\left(A_{n}\right)_{n \in \mathbb{N}}$ of $\Omega$ and some $\tilde{Z}_{n} \in \tilde{\mathcal{Z}}$ for $n \in \mathbb{N}$. 


\section{Conditional Preferences}

Proposition 4.7. A $\mathscr{G}$-conditional preference order $\succcurlyeq^{\mathscr{G}}$ which is conditionally separable admits a numerical representation.

The proof is an adaptation of the standard argumentation in the static case which can be found in [Föllmer and Schied, 2004].

Proof. Suppose that $\succcurlyeq$ is conditionally separable for the countable set $\tilde{\mathcal{Z}}$. Let $\mu$ be a counting measure on $\tilde{\mathcal{Z}}$ and $\tilde{X} \in \tilde{\mathcal{X}}$. For any $A \subset \mathscr{G}$ we further define

$$
\begin{aligned}
& \tilde{\mathcal{Z}}^{-}(\tilde{X}, A):=\left\{\tilde{Z} \in \tilde{\mathcal{Z}} \mid \tilde{Z} 1_{A} \prec_{A^{\prime}} \tilde{X} \text { for any } \mathscr{G} \text {-mesurable } A^{\prime} \subset A\right\}, \\
& \tilde{\mathcal{Z}}^{+}(\tilde{X}, A):=\left\{\tilde{Z} \in \tilde{\mathcal{Z}} \mid \tilde{Z} 1_{A} \succ_{A^{\prime}} \tilde{X} \text { for any } \mathscr{G} \text {-mesurable } A^{\prime} \subset A\right\},
\end{aligned}
$$

and

$$
\tilde{U}(\tilde{X}, A)=\sum_{\tilde{Z} \in \tilde{\mathcal{Z}}^{+}(\tilde{X}, A)} \mu(\tilde{Z})-\sum_{\tilde{Z} \in \tilde{\mathcal{Z}}^{-}(\tilde{X}, A)} \mu(\tilde{Z}),
$$

which is well defined and a real bounded by $2 \mu(\tilde{\mathcal{Z}})$. We finally set

$$
\tilde{U}(\tilde{X})=\operatorname{ess} \sup \left\{\sum_{n \in \mathbb{N}} \tilde{U}\left(\tilde{X}, A_{n}\right) 1_{A_{n}} \mid\left(A_{n}\right)_{n \in \mathbb{N}} \subset \mathscr{G} \text { is a partition of } \Omega\right\} .
$$

By definition, $\tilde{U}(\tilde{X})$ is a $\mathscr{G}$-measurable random variable, and $P$-almost surely bounded by $2 \mu(\tilde{\mathcal{Z}})$, hence an element of $\mathbb{L}^{\infty}(\Omega, \mathscr{G}, P)$. Also by definition, holds

$$
\tilde{U}\left(\tilde{X} 1_{A}\right)=\tilde{U}(\tilde{X}) 1_{A}+\tilde{U}\left(\tilde{x}_{0}\right) 1_{A^{c}} .
$$

Last, suppose that $\tilde{X} \succcurlyeq \tilde{Y}$. This means by reducibility exactly that $\tilde{\mathcal{Z}}^{-}(\tilde{Y}, A) \subset$ $\tilde{\mathcal{Z}}^{-}(\tilde{X}, A)$ and $\tilde{\mathcal{Z}}^{+}(\tilde{Y}, A) \supset \tilde{\mathcal{Z}}^{+}(\tilde{X}, A)$ for any $A \in \mathscr{G}$ and this is equivalent to $\tilde{U}(\tilde{X}, A) \geq \tilde{U}(\tilde{Y}, A)$ for any $A \in \mathscr{G}$ showing that

$$
\tilde{X} \succcurlyeq \tilde{Y} \quad \Longleftrightarrow \quad \tilde{U}(\tilde{X}) \geq \tilde{U}(\tilde{Y}), \quad P \text {-almost surely, }
$$

and therefore $\tilde{U}$ is a numerical representation of $\succcurlyeq$.

Example 4.8. The simplest example of a numerical representation which induces a $\mathscr{G}$-conditional preference is the conditional expectation

$$
\tilde{U}(\tilde{X})=E_{P}\left[\int x \tilde{X}(\cdot, d x) \mid \mathscr{G}\right]
$$

with respect to a given probability $P$ on $(\Omega, \mathscr{F})$ since condition (4.4) is clearly satisfied. $\diamond$

In the sequel, we will restrict our analyse of conditional preferences to the stochastic kernels, in the context of a conditional version of the "Variational Preferences" introduced by Maccheroni, Marinacci, and Rustichini [2006A]. 


\subsection{Conditional Variational Preferences}

Before we specify the axioms corresponding to conditional variational preferences, we describe the setting and the notations we will use from now on. The $\mathscr{G}$-conditional preference $\succcurlyeq^{\mathscr{G}}$ will be specified on the set $\mathcal{L}$ of bounded measurable functions on $(\Omega, \mathscr{F})$. One can think of $X \in \mathcal{L}$ as a scenario-dependent monetary outcome of a financial position. To make the distinction clear between what we previously called distributional risk and model risk, it will be convenient, however, to consider the conditional preference on a larger set where payoffs can be distributions. This larger convex set will be denoted $\tilde{\mathcal{X}}$, and its elements are stochastic kernels $\tilde{X}(\omega, d x)$ from $(\Omega, \mathscr{F})$ to the real line with uniform bounded support, that is, there exists $c>0$ such that:

$$
\tilde{X}(\omega,[-c, c])=1 \quad \text { for all } \omega \in \Omega \text {. }
$$

The map

$$
X \in \mathcal{L} \mapsto \delta_{X} \in \tilde{\mathcal{X}}
$$

identifies $\mathcal{L}$ with the set of all $\tilde{X} \in \tilde{\mathcal{X}}$ for which $\tilde{X}(\omega, \cdot)$ is a Dirac measure for each $\omega \in \Omega$. The reference constant $x_{0}$ of the previous section to set elements constant outside an event will be $x_{0}=\delta_{0}$, i.e., for a set $A \in \mathscr{G}$ and an element $\tilde{X} \in \tilde{\mathcal{X}}$, the notation $\tilde{X} 1_{A}$ stays for the stochastic kernel $\tilde{X} 1_{A}+\delta_{0} 1_{A^{c}}$

Although we have fixed a probability measure $P$ on $(\Omega, \mathscr{G})$, no a priori probability measure is given on the larger measurable space $(\Omega, \mathscr{F})$. Instead we consider the whole class of finitely additive set functions $Q: \mathscr{F} \rightarrow[0,1]$ which are normalized to $Q[\Omega]=$ 1. We denote this class by $\mathcal{M}_{1, f}$. With $\mathcal{M}_{1}$ we denote the $\sigma$-additive elements of $\mathcal{M}_{1, f}$, that is, the class of all probability measures on $(\Omega, \mathscr{F})$. Further, we denote by $\mathcal{M}_{1, f}(P \mid \mathscr{G}) \subset \mathcal{M}_{1 . f}$ the class of those finitely additive set functions on $(\Omega, \mathscr{F})$ which are equal to $P$ on $\mathscr{G}$, and similarly by $\mathcal{M}_{1}(P \mid \mathscr{G}) \subset \mathcal{M}_{1}$ the class of probability measures which are equal to $P$ on $\mathscr{G}$.

For any closed interval $I \subset \mathbb{R}$, we denote by $\mathcal{M}_{1, I}(\mathscr{G})$, the set of $\mathscr{G}$-measurable stochastic kernels with uniform compact support in $I$, that is, the subset of $\tilde{\mathcal{X}}$ consisting of all those stochastic kernels from $(\Omega, \mathscr{G})$ to the real line such that $\tilde{X}(\omega, I)=1$ for all $\omega \in \Omega$. Furthermore, we denote by $C_{b}(\mathscr{G})$ the set of functions $f: \Omega \times \mathbb{R} \rightarrow \mathbb{R}$ such that $\omega \mapsto f(\omega, x)$ is $\mathscr{G}$-measurable for any $x \in \mathbb{R}$ and $x \mapsto f(\omega, x)$ is uniformly bounded and continuous for $P$-almost all $\omega \in \Omega$. On the set $\mathcal{M}_{1, I}(\mathscr{G})$ we consider the $P$-almost sure weak topology:

Definition 4.9. The $P$-almost sure weak topology on $\mathcal{M}_{1, I}(\mathscr{G})$ is the coarsest topology for which all mappings:

$$
\mathcal{M}_{1, I}(\mathscr{G}) \ni \mu \mapsto \int_{\mathbb{R}} f(\omega, x) \mu(\omega, d x) \quad \text { for } f \in C_{b}(\mathscr{G})
$$

are continuous with respect to the $\|\cdot\|_{\infty}$ norm on $\mathbb{L}^{\infty}(\Omega, \mathscr{G}, P)$.

For any sequence $\left(\mu_{n}\right)_{n \in \mathbb{N}}$ in $\mathcal{M}_{1, I}(\mathscr{G})$ converging $P$-almost surely weakly to some $\mu$, we 


\section{Conditional Preferences}

write $\mu_{n} \stackrel{w, P}{\longrightarrow} \mu$. Similarly to the static case, we have a density result for the conditional simplexes of $\mathcal{M}_{1,[-c, c]}(\mathscr{G})$.

Proposition 4.10. The set of conditional convex combinations of elements of the form $\delta_{x} \nVdash_{A}+\delta_{0} \nVdash_{A^{c}}$ for $x \in[-c, c]$ and $A \in \mathscr{G}$ is dense for the P-almost sure weak topology in $\mathcal{M}_{1,[-c, c]}(\mathscr{G})$.

Proof. Take $\mu \in \mathcal{M}_{1,[-c, c]}(\mathscr{G})$ and cut the intervals $[-c, c]$, and $[0,1]$ respectively in subdivisions

$$
\sigma_{n}^{1}=\left\{-c,-c+\frac{2 c}{2^{n}}, \cdots,-c+\frac{2 c\left(2^{n}-1\right)}{2^{n}}, c\right\}, \text { and } \sigma_{n}^{2}=\left\{0, \frac{1}{2^{n}}, \cdots, \frac{2^{n}-1}{2^{n}}, 1\right\},
$$

and note

$$
\left.A_{l, k, n}=\left\{\omega \in \Omega \mid \mu(\omega,]-\infty,-c+\frac{2 c(l+1)}{2^{n}}\right]\right) \in\left[\frac{k}{2^{n}}, \frac{k+1}{2^{n}}[\},\right.
$$

for all $l, k \in \llbracket 0,2^{n}-1 \rrbracket$. Define then the measure

$$
\mu_{n}=\sum_{k, l=0}^{2^{n}-1} \nVdash_{A_{l, k, n}} \frac{1}{2^{n}} \delta_{-c+\frac{2 c(l+1)}{2^{n}}} .
$$

For any $f \in C_{b}(\mathscr{G})$, holds

$$
\int f(\omega, x) d \mu_{n}=\sum_{k, l=0}^{2^{n}-1} \nVdash_{A_{l, k, n}} \frac{1}{2^{n}} f\left(\omega,-c+\frac{2 c(l+1)}{2^{n}}\right)=\int_{\mathbb{R}} f_{n}(\omega, x) \mu(\omega, d x),
$$

whereby on $[-c, c]$,

$$
\begin{array}{r}
\left.f_{n}(\omega, x)=\sum_{k, l=0}^{2^{n}-1} \nVdash_{A_{k, l, n}} \nVdash\right]_{\left.-c+\frac{2 c l}{2^{n}},-c+\frac{2 c(l+1)}{2^{n}}\right]}(x) f\left(\omega,-c+\frac{2 c(l+1)}{2^{n}}\right) \\
\underset{n \rightarrow+\infty}{P \otimes d \lambda \text {-a.s. }} \rightarrow f(\omega, x) .
\end{array}
$$

Hence, Lebegue's dominating convergence yields

$$
\int f(\omega, x) \mu_{n}(\omega, d x) \underset{n \rightarrow+\infty}{\longrightarrow} \int f(\omega, x) \mu(\omega, d x), \quad \text { for } P \text {-almost all } \omega \in \Omega .
$$

And this is exactly the convergence of $\left(\mu_{n}\right)_{n \in \mathbb{N}}$ to $\mu$ in the $P$-almost sure weak topology.

Any binary relation $\succcurlyeq$ on $\tilde{\mathcal{X}}$ induces a natural binary relation on $\mathcal{X}, \mathcal{M}_{1, c}(\mathscr{G})$, and consequently also on $\mathcal{M}_{1, c}$. We adopt the same notation for these induced binary relations. Moreover, for $\tilde{X}, \tilde{Y} \in \tilde{\mathcal{X}}$ and $\omega \in \Omega$, the relation $\tilde{X}(\omega) \succcurlyeq \tilde{Y}(\omega)$ is understood as the induced relation on $\mathcal{M}_{1, c}$, that is, $\mu \succcurlyeq \nu$ for $\mu(d x)=\tilde{X}(\omega, d x)$ and $\nu(d x)=$ $\tilde{Y}(\omega, d x)$. 


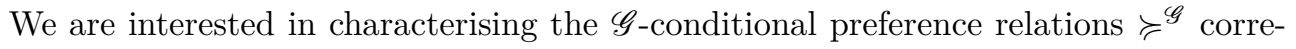
sponding to variational preferences, that is, which admit a numerical representation of the following "robust" form:

$$
\tilde{U}(\tilde{X})=-\rho\left(\int u(\cdot, x) \tilde{X}(\cdot, x)\right),
$$

where $\rho$ is a conditional convex risk measure and $u(\cdot, \cdot)$ is a monotone continuous function in its second argument and $\mathscr{G}$-measurable in its first. To this end, we introduce the following additional axioms.

A-III Risk Aversion: From $\tilde{X}, \tilde{Y} \in \tilde{\mathcal{X}}$ with $\tilde{X} \succcurlyeq^{\mathscr{G}} \tilde{Y}$, follows

$$
\alpha \tilde{X}+(1-\alpha) \tilde{Y} \succcurlyeq^{\mathscr{G}} \tilde{Y},
$$

for any $\mathscr{G}$-measurable function with values in $[0,1]$.

A-IV Monotonicity: If $\tilde{Y}(\omega) \succcurlyeq^{\mathscr{G}} \tilde{X}(\omega)$ for all $\omega \in \Omega$, then $\tilde{Y} \succcurlyeq^{\mathscr{G}} \tilde{X}$. Moreover, the preference is compatible with the order in $\mathbb{R}$, i.e., $x<y$ if and only if $\delta_{x} \prec^{\mathscr{G}} \delta_{y}$.

A-V Weak Certainty Independence: For $\tilde{X}, \tilde{Y} \in \tilde{X}, \mu_{1}, \mu_{2} \in \mathcal{M}_{1, c}(\mathscr{G}), A \in \mathscr{G}$ and any $\mathscr{G}$-measurable function $\alpha$ with $0<\alpha 1_{A} \leq 1$ holds

$$
\alpha \tilde{X}+(1-\alpha) \tilde{\mu_{1}} \succ_{A}^{\mathscr{G}} \alpha \tilde{Y}+(1-\alpha) \tilde{\mu_{1}} \Longrightarrow \alpha \tilde{X}+(1-\alpha) \tilde{\mu_{2}} \succ_{A}^{\mathscr{G}} \alpha \tilde{Y}+(1-\alpha) \tilde{\mu_{2}} .
$$

A-VI Continuity: If $\tilde{X}, \tilde{Y}, \tilde{Z} \in \tilde{\mathcal{X}}$ are such that $\tilde{Z} \succ_{A}^{\mathscr{G}} \tilde{Y} \succ_{A}^{\mathscr{G}} \tilde{X}$ for some $A \in \mathscr{G}$, there exist $\mathscr{G}$-measurable functions $\alpha, \beta$ with values in $(0,1)$ on $A$ such that

$$
\alpha \tilde{Z}+(1-\alpha) \tilde{X} \quad \succ_{A}^{\mathscr{G}} \quad \tilde{Y} \quad \succ_{A}^{\mathscr{G}} \quad \beta \tilde{Z}+(1-\beta) \tilde{X}
$$

Moreover, the restriction of $\succcurlyeq^{\mathscr{G}}$ to $\mathcal{M}_{1,[-c, c]}(\mathscr{G})$ is continuous with respect to the $P$-almost surely weak topology for all $c>0$.

This set of axioms A-III to A-VI is the conditional version of the axioms introduced by Maccheroni et AL. [2006A]; if the $\sigma$-algebra is $\mathscr{G}$ trivial then the axioms are the same. The continuity axiom is a very common one in the theory of choice and sometimes called the Archimedean axiom. The monotonicity axiom states that if the lottery $\tilde{X}(\omega, \cdot)$ is preferred to the lottery $\tilde{Y}(\omega, \cdot)$ for any scenario $\omega \in \Omega$, then the stochastic kernel $\tilde{X}$ has to be preferred to the stochastic kernel $\tilde{Y}$ as a whole. Uncertainty aversion in the form A-III extends the assumption of uncertainty independence introduced by SAVAGE [1972], where $\succcurlyeq$ is replaced by $\sim$. For additional information about the economic interpretation of these axioms and illustrative examples in the unconditional case, we refer to [Föllmer and Penner, 2006, Maccheroni et al., 2006a, Föllmer et al., 2009].

Note that all axioms are satisfied if $\succcurlyeq^{\mathscr{G}}$ is represented by a functional $\tilde{U}$ of the form (4.5). This is easily verified for axioms A-III, A-IV, and A-V. For A-VI we refer to the 


\section{Conditional Preferences}

proof of Theorem 5.5. 


\section{Conditional Robust Representations}

\subsection{A Conditional version of the von Neumann and Morgenstern Representation}

In this section we extend the classical representation in terms of expected utility of von Neumann and Morgenstern to the conditional case. A related extension was already discussed by KREPS AND PORTEus [1979] in the setting of ranked lotteries which depend on some parameter, but this does not involve measurability questions which are crucial in our case.

Let us consider a $\mathscr{G}$-conditional preference relation $\succcurlyeq^{\mathscr{G}}$ restricted to the set of $\mathscr{G}$ measurable stochastic kernels $\mathcal{M}_{1, c}(\mathscr{G})$ which satisfies the axioms A-IV, A-V and A-VI. On this level, the axiom $\mathrm{A}-\mathrm{V}$ of weak certainty independence reduces to the following conditional version of the independence axiom:

$\mathrm{A}-\mathrm{V}^{\prime}$ Independence: For any $\mu, \nu \in \mathcal{M}_{1, c}(\mathscr{G}), A \in \mathscr{G}$ and any $\mathscr{G}$-measurable function $\alpha$ with $0<\alpha 1_{A} \leq 1$, the relation $\mu \succ_{A}^{\mathscr{G}} \nu$ implies:

$$
\alpha \mu+(1-\alpha) \lambda \succ_{A}^{\mathscr{G}} \alpha \nu+(1-\alpha) \lambda \quad \text { for any } \lambda \in \mathcal{M}_{1, c}(\mathscr{G})
$$

Theorem 5.1. For a $\mathscr{G}$-conditional preference relation on $\mathcal{M}_{1, c}(\mathscr{G})$ satisfying axiom $A-I V, A-V$ and $A-V I$, there exists a conditional von Neumann and Morgenstern representation

$$
U(\omega, \mu)=\int u(\omega, x) \mu(\omega, d x) \quad \text { for P-allmost any } \omega \in \Omega
$$

where $u(\omega, \cdot)$ is continuous and non decreasing for P-almost all $\omega \in \Omega$ and $u(\cdot, x)$ is $\mathscr{G}$-measurable for all $x \in \mathbb{R}$. In particular, the random variable $U(\cdot, \mu)$ is $\mathscr{G}$-measurable.

Conversely, such a numerical representation (5.1) induces a $\mathscr{G}$-conditional preference relation on $\mathcal{M}_{1, c}(\mathscr{G})$ satisfying axioms $A-I V, A$ - $V^{\prime}$ and $A$-VI restricted to $\mathcal{M}_{1, c}(\mathscr{G})$.

Remark 5.2. The monotonicity axiom A-IV is not necessary if we drop the requirement that $u(\cdot, \omega)$ is non decreasing. But it simplifies the proof of the existence of $u$.

The proof of the theorem is straightforward if $\mathscr{G}$ has countably many atoms, by applying the classical result separately on each atom. In our general setting, the arguments are more subtle. 


\section{Conditional Robust Representations}

In the following, for $\mu, \nu \in \mathcal{M}_{1, c}(\mathscr{G})$ we define

$$
\sup \{\mu, \nu\}=\mu 1_{A \cup C}+\nu 1_{B} \quad \text { and } \quad \inf \{\mu, \nu\}=\mu 1_{B \cup C}+\nu 1_{A},
$$

where $A, B, C \in \mathscr{G}$ is the maximal partition introduced in Lemma 4.5, with $\mu \succ_{A}^{\mathscr{G}} \nu$, $\mu \prec_{B}^{\mathscr{G}} \nu$ and $\mu \sim_{C}^{\mathscr{G}} \nu$.

Before moving on to the proof, we state first a conditional version of the Lemma 2.24 in [Föllmer and Schied, 2004].

Lemma 5.3. Under the assumptions of the Theorem 5.1 the following properties hold:

(i) If $\mu \succ_{A}^{\mathscr{G}} \nu$ for some set $A \in \mathscr{G}$, then for any $\mathscr{G}$-measurable functions $\alpha, \beta$ with $0 \leq \alpha 1_{A}<\beta 1_{A} \leq 1$ :

$$
\beta \mu+(1-\beta) \nu \succ_{A}^{\mathscr{G}} \alpha \mu+(1-\alpha) \nu
$$

(ii) If $\mu \succ_{A}^{\mathscr{G}} \nu$ and $\mu \succcurlyeq_{A}^{\mathscr{G}} \lambda \succcurlyeq_{A}^{\mathscr{G}} \nu$ for some set $A \in \mathscr{G}$, then there exists a $\mathscr{G}$-measurable function $\alpha$ with values in $[0,1]$ such that:

$$
\lambda \sim_{A}^{\mathscr{G}} \alpha \mu+(1-\alpha) \nu
$$

and $\alpha$ is P-almost surely uniquely determined on $A$.

(iii) If $\mu \sim_{A}^{\mathscr{G}} \nu$ for some set $A \in \mathscr{G}$, then for any $\lambda \in \mathcal{M}_{1, c}(\mathscr{G})$ and $\mathscr{G}$-measurable function $\alpha$ with values in $[0,1]$ :

$$
\alpha \mu+(1-\alpha) \lambda \sim_{A}^{\mathscr{G}} \alpha \nu+(1-\alpha) \lambda
$$

Proof (Proof). The proof of this lemma uses classical arguments in choice theory, but it needs some additional care in dealing with measurability issues. For notational convenience, we prove (i),(ii) and (iii) for $A=\Omega$, the argumentation being exactly the same for general $A \in \mathscr{G}$.

(i) Define $\lambda=\beta \mu+(1-\beta) \nu$. The conditional independence axiom A- $\mathrm{V}^{\prime}$ implies that $\lambda \succ \beta \nu+(1-\beta) \nu=\nu$. Since $\beta$ is strictly positive $P$-almost surely, we can use again the conditional independence axiom with $\gamma=\alpha / \beta$ to conclude that:

$$
\beta \mu+(1-\beta) \nu=\gamma \lambda+(1-\gamma) \lambda \succ \gamma \lambda+(1-\gamma) \nu=\alpha \mu+(1-\alpha) \nu
$$

(ii) Concerning uniqueness, suppose that relation (5.3) holds for $\alpha$ and $\alpha^{\prime}$. Consider the set $A=\left\{\alpha<\alpha^{\prime}\right\}$ (resp $B=\left\{\alpha>\alpha^{\prime}\right\}$ ) and apply (i) to see that relation (5.2) holds for $\beta=\alpha^{\prime}$ over $A$ (resp $\beta=\alpha$ over $B$ ) and together with (5.3) implies $P[A]=P[B]=0$ and consequently $\alpha=\alpha^{\prime} P$-almost surely. 


\subsection{A Conditional version of the von Neumann and Morgenstern Representation}

In order to show existence, consider the set

$$
\mathcal{A}=\left\{\beta \in \mathbb{L}^{\infty}(\Omega, \mathscr{G}, P) \mid 0 \leq \beta \leq 1 \text { and } \lambda \succcurlyeq \beta \mu+(1-\beta) \nu\right\} .
$$

It is non empty because $0 \in \mathcal{A}$. Our candidate will naturally be the essential supremum of $\mathcal{A}$ w.r.t. $P$ denoted by $\alpha=\operatorname{ess} \sup \mathcal{A}$. Using lemma 4.5 , we obtain the existence of a partition $A, B, C \in \mathscr{G}$ of $\Omega$, such that

$$
\lambda \succ_{A} \alpha \mu+(1-\alpha) \nu \quad ; \quad \lambda \prec_{B} \alpha \mu+(1-\alpha) \nu \quad \text { and } \quad \lambda \sim_{C} \alpha \mu+(1-\alpha) \nu .
$$

Suppose first that $P[A]>0$. Using the continuity axiom A-VI, there exists a $\mathscr{G}$-measurable function $\beta$ with $0<\beta 1_{A}<1$ such that

$$
\begin{array}{r}
\lambda \succ_{A} \beta \mu+(1-\beta)[\alpha \mu+(1-\alpha) \nu]=[\beta+(1-\beta) \alpha] \mu+(1-\beta)(1-\alpha) \nu \\
=\tilde{\alpha} \mu+(1-\tilde{\alpha}) \nu
\end{array}
$$

where $\tilde{\alpha}=(\beta+(1-\beta) \alpha) 1_{A}+\alpha 1_{A^{c}}$. It is easy to verify that $\tilde{\alpha} 1_{A}>\alpha 1_{A}$ and that $\tilde{\alpha} \in \mathcal{A}$. This contradicts the definition of $\alpha$ as an essential supremum, and so we get $P[A]=0$.

Suppose now that $P[B]>0$. Using the continuity axiom A-VI, we get again the existence of a $\mathscr{G}$-measurable function $\beta$ such that $0<\beta 1_{B}<1$ with the property

$$
\lambda \prec_{B}(1-\beta) \nu+\beta[\alpha \mu+(1-\alpha) \nu]=\beta \alpha \mu+(1-\beta \alpha) \nu=\tilde{\alpha} \mu+(1-\tilde{\alpha}) \nu
$$

where $\tilde{\alpha}=\beta \alpha 1_{B}+\alpha 1_{B^{c}}$. Over $B, \tilde{\alpha}$ is strictly smaller than $\alpha$, thus (i) and the definition of $\alpha$ as an essential supremum implies that $\tilde{\alpha}$ belongs to $\mathcal{A}$. In view of relation (5.5) this implies $P[B]=P[A]=0$, and this shows $\lambda \sim_{\Omega} \alpha \mu+(1-\alpha) \nu$.

(iii) We can suppose that $\alpha>0$, because on the set $\{\alpha=0\}$ the property is immediate. As before, there exists a maximal partition $A, B, C \in \mathcal{G}$ such that:

$$
\begin{aligned}
& \alpha \mu+(1-\alpha) \lambda \prec_{A} \alpha \nu+(1-\alpha) \lambda, \\
& \alpha \mu+(1-\alpha) \lambda \succ_{B} \alpha \nu+(1-\alpha) \lambda, \\
& \alpha \mu+(1-\alpha) \lambda \sim_{C} \alpha \nu+(1-\alpha) \lambda .
\end{aligned}
$$

If every $\rho \in \mathcal{M}_{1, c}(\mathscr{G})$ satisfies $\rho \sim_{A} \mu \sim_{A} \nu$, then $P[A]=0$. If not, then exists $\tilde{\rho} \in \mathcal{M}_{1, c}(\mathscr{G})$ such that $\tilde{\rho} \chi_{A} \mu \operatorname{implying} \sup \{\tilde{\rho}, \mu\} \succ_{A} \mu$ or $\inf \{\tilde{\rho}, \mu\} \prec_{A} \mu$.

Suppose $\rho=\sup \{\tilde{\rho}, \mu\} \succ_{A} \mu$. From the independence axiom A-V', we have for any $\mathscr{G}$-measurable function $\beta$ with $0<\beta 1_{A} \leq 1$ :

$$
\beta \rho+(1-\beta) \mu \succ_{A} \mu \sim_{A} \nu
$$

Using again the independence axiom with $\alpha$ (which is strictly positive) and relation 


\section{Conditional Robust Representations}

(5.6) yields

$$
\alpha[\beta \rho+(1-\beta) \mu]+(1-\alpha) \lambda \succ_{A} \alpha \nu+(1-\alpha) \lambda .
$$

From $(i i)$, there exists a $\mathscr{G}$-measurable function $\gamma$ with values in $[0,1]$ such that

$$
\begin{aligned}
\alpha \nu+(1-\alpha) \lambda \sim_{A} \gamma[\alpha[\beta \rho+(1-\beta) \mu]+ & (1-\alpha) \lambda]+(1-\gamma)[\alpha \mu+(1-\alpha) \lambda] \\
& =\alpha[\beta \gamma \rho+(1-\beta \gamma) \nu]+(1-\alpha) \lambda .
\end{aligned}
$$

Because of the maximality of the partition and (5.6) we have $\gamma 1_{A}>0$. Hence $\beta \gamma 1_{A}>0$, and using again relations (5.7) and (5.8) for $\beta \gamma$ instead of $\beta$ yields

$$
\alpha \mu+(1-\alpha) \lambda \sim_{A} \alpha[\beta \gamma \rho+(1-\beta \gamma) \nu]+(1-\alpha) \lambda \succ_{A} \alpha \nu+(1-\alpha) \lambda .
$$

In view of (5.6) this implies $P[A]=0$. The same argument holds for the case where $\rho=\inf \{\mu, \nu\} \prec_{A} \nu$, implying again that $P[A]=0$.

In the same way we obtain $P[B]=0$, and this ends the demonstration.

Proof (of Theorem 5.1). The monotonicity axiom A-IV insures the existence of some $\mu, \nu \in \mathcal{M}_{1, c}(\mathscr{G})$ with $\mu \succ \nu$. We begin with the existence of a numerical representation $U$ on the set

$$
\mathcal{M}(\mathscr{G}, \mu, \nu)=\left\{\lambda \in \mathcal{M}_{1, c}(\mathscr{G}) \mid \mu \succcurlyeq \lambda \succcurlyeq \nu\right\} .
$$

According to lemma 5.3, there exists for any $\lambda \in \mathcal{M}(\mu, \nu, \mathscr{G})$ a unique $\mathscr{G}$-measurable function $\alpha$ with value in $[0,1]$ such that

$$
\lambda \sim \alpha \mu+(1-\alpha) \nu
$$

The functional $U(\lambda):=\alpha$ is thus well defined on $\mathcal{M}(\mathscr{G}, \mu, \nu)$.

For $\eta, \lambda \in \mathcal{M}(\mu, \nu, \mathscr{G})$, over $A=\{U(\eta)=U(\lambda)\}$ we have by definition of $U$ clearly $\eta \sim_{A} \lambda$. Due to (i) in (5.3), for $B=\{U(\eta)>U(\lambda)\}$ holds $\eta \succ_{B} \lambda$, hence $\eta \succcurlyeq \lambda$ implies $U(\eta) \geq U(\lambda)$. Reciprocally, suppose $\eta \succcurlyeq \lambda$ and take the maximal partition from (4.5) such that $\eta \succ_{A} \lambda$ and $\eta \sim_{C} \lambda$ with $A \cup C=\Omega$. Again, from definition of $U$ holds $U(\eta) 1_{C}=U(\lambda) 1_{A}$ and by an immediate contradiction argument using (i) of 5.3 we get $\{U(\eta)<U(\lambda)\} \cap A=\{U(\eta)<U(\lambda)\} \cap C=\emptyset P$-almost surely, hence $B=\emptyset$ implying that $U(\eta) \geq U(\lambda)$. This shows that $U$ is a conditional representation of $\succcurlyeq$ restricted to $\mathcal{M}(\mu, \nu, \mathscr{G})$.

From the independence axiom A- $\mathrm{V}^{\prime}$ together with point (iii) of Lemma 5.3, the set $\mathcal{M}(\mathscr{G}, \mu, \nu)$ is conditionally convex. Hence $U(\alpha \eta+(1-\alpha) \lambda)$ is well defined for any $\eta, \lambda \in \mathcal{M}(\mathscr{G}, \mu, \nu)$ and $\mathscr{G}$-measurable function $\alpha$ with values in [0,1]. Applying (iii) in 
5.3 yields

$$
\begin{aligned}
\alpha \eta+(1-\alpha) \lambda & \sim \alpha(U(\eta) \mu+(1-U(\eta)) \nu)+(1-\alpha)(U(\lambda) \mu+(1-U(\lambda)) \nu) \\
& \sim(\alpha U(\eta)+(1-\alpha) U(\lambda)) \mu+(1 \alpha U(\eta)-(1-\alpha) U(\lambda)) \nu .
\end{aligned}
$$

Uniqueness in point $(i i)$ of 5.3 and the definition of $U$ yield

$$
U(\alpha \eta+(1-\alpha) \lambda)=\alpha U(\eta)+(1-\alpha) U(\lambda)
$$

Hence, $U$ is affine.

Consider an affine numerical representation $\tilde{U}$ of $\succcurlyeq$ and define

$$
\hat{U}(\lambda)=\frac{\tilde{U}(\lambda)-\tilde{U}(\nu)}{\tilde{U}(\mu)-\tilde{U}(\nu)}, \quad \lambda \in \mathcal{M}(\mu, \nu, \mathscr{G}),
$$

which is a positive affine transformation of $\tilde{U}$ with $\hat{U}(\mu)=1$ and $\hat{U}(\nu)=0$. Using the definition of $U$, we get

$$
\hat{U}(\lambda)=\hat{U}(U(\mu) \mu+(1-U(\nu)) \nu)=U(\mu) \hat{U}(\nu)+(1-U(\nu)) \hat{U}(\nu)=U(\lambda)
$$

Thus $\hat{U}=U$ and $U$ is up to any positive affine transformation a unique affine numerical representation of $\succcurlyeq$ restricted to $\mathcal{M}(\mathscr{G}, \mu, \nu)$.

Since for any two elements $\mu, \nu \in \mathcal{M}_{1, c}(\mathscr{G})$, hold $\sup \{\mu, \nu\} \succcurlyeq \mu, \nu \succcurlyeq \inf \{\mu, \nu\}$, we have

$$
\mathcal{M}_{1, c}(\mathscr{G})=\bigcup_{\mu \succ \nu} \mathcal{M}(\mu, \nu, \mathscr{G})
$$

It remains to show that this normalized numerical representation of $\succcurlyeq$ restricted to $\mathcal{M}(\mathscr{G}, \mu, \nu)$ extends uniquely to a conditional representation of $\succcurlyeq$ restricted to the bigger $\mathcal{M}(\mathscr{G}, \tilde{\mu}, \tilde{\nu})$ for $\tilde{\mu} \succcurlyeq \mu \succ \nu \succcurlyeq \tilde{\nu}$. But this follows immediately from the existence and uniqueness arguments given for $\mathcal{M}(\mathscr{G}, \mu, \nu)$ applied to $\mathcal{M}(\mathscr{G}, \tilde{\mu}, \tilde{\nu})$.

We now have an affine numerical representation $U$ for $\succcurlyeq \operatorname{restricted~to~} \mathcal{M}_{1, c}(\mathscr{G})$. It remains to show the explicit part of the representation (5.1) by defining $u$ and checking its properties. We can assume, up to a strictly positive affine transformation, that $U\left(\delta_{0}\right)=0$ P-almost surely.

The numerical representation $U(\mu)$ being only defined $P$-almost surely for any $\mu \in$ $\mathcal{M}_{1, c}(\mathscr{G})$, we cannot simply set $u(\omega, x):=U\left(\delta_{x}\right)(\omega)$ for $x, \omega \in \mathbb{R} \times \Omega$ as in the unconditional case. There exists however a set $N$ of $P$-null measure in $\mathscr{G}$ such that $u(q, \omega):=U\left(\delta_{q}\right)(\omega)$ is well defined for any $q \in \mathbb{Q}$ and $\omega \in \Omega \backslash N$. From the monotonicity axiom, it is possible to define the following functional continuous from the right

$$
u(\cdot, x):=\lim _{\substack{q \searrow x \\ q \in \mathbb{Q}}} u(\cdot, q) \quad \text { for any real } x .
$$

For any rational $q, U\left(\delta_{q}\right)$ is $\mathscr{G}$-measurable and so is $u(\cdot, x)$ for any real $x$. Let us check 


\section{Conditional Robust Representations}

that $u$ is in fact continuous $P$-almost surely.

To do so, we show in a first step that for any $\mathscr{G}$-measurable bounded random variables $\tau$ and $\tau^{(n)}$ with $\tau^{(n)} \searrow \tau\left(\operatorname{resp} \tau^{(n)} \nearrow \tau\right) P$-almost surely then $U\left(\delta_{\tau^{(n)}}\right)$ converges also to $U\left(\delta_{\tau}\right) P$-almost surely. Indeed, it is clear that $\delta_{\tau^{(n)}} \stackrel{w, P}{\longrightarrow} \delta_{\tau}$, and by monotonicity that $U\left(\delta_{\tau^{(n)}}\right)$ is monotone decreasing and greater than $U\left(\delta_{\tau}\right)$. Write $Z=\lim \downarrow U\left(\delta_{\tau^{(n)}}\right)$ and suppose that $\left\{Z>U\left(\delta_{\tau}\right)\right\}$ has positive measure. There exists then some $\varepsilon>0$, some set of strict positive measure $A_{\varepsilon}$, and some $n_{0} \in \mathbb{N}$ such that on $A_{\varepsilon}$ holds

$$
U\left(\delta_{\tau}\right)+\varepsilon \leq Z \leq U\left(\delta_{\tau^{(n)}}\right) \leq Z+\frac{\varepsilon}{2},
$$

for any $n \geq n_{0}$. If we define

$$
\mu_{\varepsilon}=\left(\frac{1}{2} \delta_{\tau}+\frac{1}{2} \delta_{\tau^{\left(n_{0}\right)}}\right) 1_{A_{\varepsilon}}+\delta_{\tau} 1_{A_{\varepsilon}^{c}},
$$

then clearly $\mu_{\varepsilon} \succ \delta_{\tau}$. But on the other hand, by construction, holds on $A_{\varepsilon}$

$$
U\left(\mu_{\varepsilon}\right)=\frac{U\left(\delta_{\tau}\right)}{2}+\frac{U\left(\delta_{\tau^{\left(n_{0}\right)}}\right)}{2} \leq \frac{Z}{2}-\frac{\varepsilon}{2}+\frac{Z}{2}+\frac{1}{4 \varepsilon}=Z-\frac{\varepsilon}{4}<U\left(\delta_{\left.\tau^{(n)}\right)}\right),
$$

for any $n \geq n_{0}$, showing that $\delta_{\tau^{(n)}} \succ \mu \succ \delta_{\tau}$. This contradicts however the continuity of $\succcurlyeq$ on $\mathcal{M}_{1,[-c, c]}(\mathscr{G})$ with respect to the $P$-almost sure weak topology and the fact that $\delta_{\tau^{(n)}} \stackrel{w, P}{\longrightarrow} \delta_{\tau}$. Hence $U\left(\delta_{\tau^{(n)}}\right) \rightarrow U\left(\delta_{\tau}\right) P$-almost surely and the same result also holds with the same argumentation for monotone increasing $\tau^{(n)} \nearrow \tau P$-almost surely.

In a second step, we show that $U\left(\delta_{\tau}\right)=u(\cdot, \tau(\cdot)) P$-almost surely and this for any $\mathscr{G}$-measurable bounded random variable $\tau$. The affine property of $U$ shows that for $\tau=\sum_{i=1}^{n} \delta_{q_{i}} 1_{A_{i}}$ where the $q_{i}$ are rationals and the sets $A_{i} \in \mathscr{G}$ build a partition of $\Omega$ we have

$$
U\left(\delta_{\tau}\right)=\sum_{i=1}^{n} U\left(\delta_{q_{i}}\right) 1_{A_{i}}=u(\cdot, \tau) .
$$

For a general bounded $\mathscr{G}$-measurable random variable $\tau$, consider a decreasing sequence $\tau^{(n)}$ of the previous type, monotone decreasing to $\tau$. For any $n \in \mathbb{N}, U\left(\delta_{\tau^{(n)}}\right)=$ $u\left(\cdot, \tau^{(n)}\right)$. Moreover, from the definition of $u$ as decreasing limits over the rational, we have $u(\cdot, \tau)=\lim \downarrow u\left(\cdot, \tau^{(n)}\right) P$-almost surely. Lastly, because $\delta_{\tau^{(n)}} \stackrel{w, P}{\longrightarrow} \delta_{\tau}$ and the previous step, we have $U\left(\delta_{\tau}\right)=\lim \downarrow U\left(\delta_{\tau^{(n)}}\right)$. Hence $U\left(\delta_{\tau}\right)=u(\cdot, \tau)$.

In the third step, we show that the functional $u$ is indeed continuous $P$-almost surely. To do so, we fix some rational $q>0$ and $\varepsilon>0$ and define the first point after $-q$ where a jump of size $\varepsilon$ occurs, that is

$$
\tau_{q, \varepsilon}:=\inf \{x>-q \mid u(\cdot, x)-u(\cdot, x-) \geq \varepsilon\} .
$$

with convention that $\inf \emptyset=+\infty$. We are interested in the set of first jumps of size $\varepsilon$ 
in the interval $[-q, q]$, i.e.,

$$
\begin{aligned}
A_{q, \varepsilon} & =\left\{\tau_{q, \varepsilon} \leq q\right\} \\
& =\bigcap_{n \in \mathbb{N}^{*}} \bigcup_{r, s \in \mathbb{Q}}\left\{u(\cdot, s)-u(\cdot, r) \geq \varepsilon \mid-q \leq r<s \leq q \text { and }|s-r|<\frac{1}{n}\right\} \in \mathscr{G} .
\end{aligned}
$$

Suppose that $P\left[A_{q, \varepsilon}\right]>0$. Consider then $\tau_{q, \varepsilon}^{(n)}=-q / n+(1-1 / n) \tau_{q, \varepsilon}$ to define

$$
\mu_{q, \varepsilon}^{(n)}=\delta_{\tau_{q, \varepsilon}^{(n)}} 1_{A_{q, \varepsilon}}+\delta_{-q} 1_{A_{q, \varepsilon}^{c}} \in \mathcal{M}_{1,[-q, q]}(\mathscr{G}) .
$$

By definition holds clearly $\mu_{\varepsilon, q}^{(n)} \stackrel{w, P}{\longrightarrow} \mu_{q, \varepsilon}$ where

$$
\mu_{q, \varepsilon}=\delta_{\tau_{q, \varepsilon}} 1_{A_{q, \varepsilon}}+\delta_{-q} 1_{A_{q, \varepsilon}^{c}} \in \mathcal{M}_{1,[-q, q]}(\mathscr{G})
$$

On the other side, by construction of $\tau_{q, \varepsilon}$ and the results of the second step, we have on $A_{q, \varepsilon}$

$$
U\left(\mu_{q, \varepsilon}^{(n)}\right)=u\left(\cdot, \tau_{q, \varepsilon}^{(n)}\right) \leq u\left(\cdot, \tau_{q, \varepsilon}\right)-\varepsilon=U\left(\mu_{q, \varepsilon}\right)-\varepsilon .
$$

But this inequality contradicts the first step where from $\tau_{q, \varepsilon}^{(n)} \nearrow \tau_{q, \varepsilon}$ over $A_{q, \varepsilon}$ should follow $U\left(\delta_{\tau^{(n)}}\right) 1_{A_{q, \varepsilon}} \rightarrow U\left(\delta_{\tau_{q, \varepsilon}}\right) P$-almost surely. Hence $P\left[A_{q, \varepsilon}\right]=0$ for any $\varepsilon>0$ and rational $q>0$. This ends the proof that $u$ is continuous in its second component $P$-almost surely.

Finaly, knowing that the set of conditional convex combination of elements of the form $\delta_{x} 1_{A}+\delta_{0} 1_{A^{c}}$ for $x \in[-c, c]$ and $A \in \mathscr{G}$ is dense for the $P$-almost sure weak topology in $\mathcal{M}_{1,[-c, c]}(\mathscr{G})$, see Proposition 4.10, the continuity of $U$ restricted to this set with respect to this topology yields a representation of the form $(5.1)$ over $\mathcal{M}_{1,[-c, c]}(\mathscr{G})$

The extension of this representation over $\mathcal{M}_{1, c}(\mathscr{G})$ holds by an extension argument over $\mathcal{M}_{1, c}(\mathscr{G})=\bigcup_{c \in \mathbb{R}^{+}} \mathcal{M}_{1,[-c, c]}(\mathscr{G})$.

Let us finally shows that the representation 5.1 induces a $\mathscr{G}$-conditional preference order on $\mathcal{M}_{1, c}(\mathscr{G})$ satisfying the axiom A-IV, A-V and A-VI. Consider such a numerical representation and the induced binary relation $\mu \succcurlyeq \nu$ if and only if $U(\mu) \geq U(\nu) P_{-}$ almost surely. This clearly defines a $\mathscr{G}$-conditional preference order satisfying axiom AIV and A-V. In order to verify the continuity axiom A-VI, we consider $\mu, \nu, \lambda \in \mathcal{M}_{1, c}(\mathscr{G})$ and $A \in \mathscr{G}$ such that

$$
U(\mu) 1_{A}>U(\lambda) 1_{A}>U(\nu) 1_{A} .
$$

The $\mathscr{G}$-measurable function

$$
\gamma=\left(\frac{U(\lambda) 1_{A}-U(\nu) 1_{A}}{U(\mu) 1_{A}-U(\nu) 1_{A}}\right) 1_{A},
$$

takes values in $(0,1)$ on $A$. Defining $\alpha=(1-\gamma) / 2$ and $\beta=\gamma / 2$, they both take value 
in $(0,1)$ on $A$. Moreover

$$
\begin{aligned}
\alpha U(\mu) 1_{A}+(1-\alpha) U(\nu) 1_{A}>\gamma U(\mu) 1_{A}+(1-\gamma) U(\nu) 1_{A}=\lambda & \\
& >\beta U(\mu) 1_{A}+(1-\beta) U(\nu) 1_{A},
\end{aligned}
$$

hence:

$$
\alpha \mu+(1-\alpha) \nu \succ_{A} \lambda \succ_{A} \beta \mu+(1-\beta) \nu
$$

In order to verify $P$-almost sure weak continuity, we fix some real $c>0$ and some $\mu \in$ $\mathcal{M}_{1,[-c, c]}(\mathscr{G})$ and consider the set $\left\{\nu \in \mathcal{M}_{1,[-c, c]}(\mathscr{G}) \mid \nu \succcurlyeq \mu\right\}$. Since $u$ is continuous and bounded on each interval $[-c, c] P$-almost surely, the restriction of $u$ to $[-c, c]$ belongs to $C_{b}(\mathscr{G})$, and so the set

$$
\begin{aligned}
\left\{\nu \in \mathcal{M}_{1,[-c, c]}(\mathscr{G}) \mid \nu \succcurlyeq \mu\right\} & \\
=\left\{\nu \in \mathcal{M}_{1,[-c, c]}(\mathscr{G}) \mid \int u(x, \cdot) \nu(\cdot, d x) \geq \int u(x, \cdot) \mu(\cdot, d x)\right\}, &
\end{aligned}
$$

is closed with respect to the $P$-almost sure topology.

The same argumentation holds for the set $\left\{\nu \in \mathcal{M}_{1,[-c, c]}(\mathscr{G}) \mid \nu \preccurlyeq \mu\right\}$ and this shows the continuity of $\succcurlyeq$ restricted to $\mathcal{M}_{1,[-c, c]}(\mathscr{G})$ for the $P$-almost sure weak topology.

\subsection{Robust Representation of the Conditional Variational Preferences}

In this section, we state the conditional version of the robust representation of preferences in [Maccheroni et al., 2006a, Föllmer et al., 2009]. Even in its unconditional version ( $\mathscr{G}$ is the trivial $\sigma$-algebra), our Theorem 5.5 is slightly more general than in [Maccheroni et al., 2006a, Föllmer et al., 2009] because our robust representation relies only on the axioms above, and not on the additional assumption that the range of $u$ is unbounded.

Before we move on to the theorem, we recall the definition of a conditional cash additive risk measure. Recall from the first chapter that the concept of unconditional cash additive risk measures was introduced by ARTZNER ET AL. [1999] and extended to the convex case by Föllmer AND Schied [2002]. Conditional cash additive risk measures were introduced in Detlefsen and Scandolo [2005]; They are crucial in the analysis of dynamic risk measures. For simplicity we refer to Föllmer and Penner [2006] and the references therein.

Definition 5.4. A functional $\rho: \mathcal{L} \rightarrow \mathbb{L}^{\infty}(\Omega, \mathscr{G}, P)$ is called a conditional cash additive risk measure if it satisfies the following properties for all $X, Y \in \mathcal{L}$ : 
- Conditional Translation Invariance: For any $m \in \mathbb{L}(\Omega, \mathscr{F}, P)$ holds

$$
\rho(X+m)=\rho(X)-m .
$$

- Monotonicity: $X \leq Y$ implies $\rho(X) \geq \rho(Y)$

- Conditional convexity: For any $\mathscr{G}$-convex coefficient $\alpha$ :

$$
\rho(\alpha X+(1-\alpha) Y) \leq \alpha \rho(X)+(1-\alpha) \rho(Y)
$$

- Normalization: $\rho(0)=0$.

A conditional cash additive risk measure is said to be coherent if the following additional property holds:

- Coherence: $\rho(\alpha X)=\alpha \rho(X)$ for any positive bounded $\mathscr{G}$-measurable random variable $\alpha$.

By $\overline{\mathbb{L}}_{+}^{0}(\Omega, \mathscr{G}, P)$, we denote the set of $\mathscr{G}$-measurable function $Z: \Omega \rightarrow[0,+\infty]$.

Theorem 5.5. Given a $\mathscr{G}$-conditional preference relation $\succcurlyeq^{\mathscr{G}}$ fulfilling axioms $A-I$ to $A$-VI, there exists a numerical representation $\tilde{U}$ given by

$$
\tilde{U}(\tilde{X})=-\rho\left(\int u(\cdot, x) \tilde{X}(\cdot, d x)\right), \quad \tilde{X} \in \tilde{\mathcal{X}}
$$

where $u: \Omega \times \mathbb{R} \rightarrow \mathbb{R}$ is $\mathscr{G}$-measurable in its first term for each $x \in \mathbb{R}$, monotone continuous in its second term $P$-almost surely and where $\rho$ is a $\mathscr{G}$-conditional cash additive risk measure on $\mathcal{L}$ uniquely determined by $u$.

In particular $\tilde{U}$ can be represented in terms of penalty function:

$$
\tilde{U}(\tilde{X})=\underset{Q \in \mathcal{M}_{1, f}(P \mid \mathscr{G})}{\operatorname{essinf}}\left\{E_{Q}\left[\int u(\cdot, x) \tilde{X}(\cdot, d x) \mid \mathscr{G}\right]+\alpha_{m i n}^{\mathscr{G}}(Q)\right\}, \quad \tilde{X} \in \tilde{\mathcal{X}},
$$

where $\alpha_{\text {min }}^{\mathscr{G}}: \mathcal{M}_{1, f}(P \mid \mathscr{G}) \rightarrow \overline{\mathbb{L}}_{+}^{0}(\Omega, \mathscr{G}, P)$.

Conversely, a numerical representation of the type 5.10 induces a $\mathscr{G}$-conditional preference relation $\succcurlyeq^{\mathscr{G}}$ fulfilling axiom A-I to A-VI.

Moreover, if the range of $u$ is $P$-almost surely unbounded, and if the restriction of $\succcurlyeq^{\mathscr{G}}$ to $\mathcal{L}$, viewed as a subset of $\tilde{\mathcal{X}}$ satisfies the following regularity condition:

- Continuity from below: For any $X, Y \in \mathcal{L}$ with $X(\omega)>Y(\omega)$ for all $\omega \in \Omega$ holds

$$
X_{n} \nearrow X \quad \Longrightarrow \quad X_{n} \succcurlyeq^{\mathscr{G}} Y \text { for all large } n
$$




\section{Conditional Robust Representations}

then the penalty function $\alpha_{\text {min }}^{\mathscr{G}}$ is concentrated on $\mathcal{M}_{1}(P \mid \mathscr{G})$, i.e.:

$$
\tilde{U}(\tilde{X})=\underset{Q \in \mathcal{M}_{1}(P \mid \mathscr{G})}{\operatorname{essinf}}\left\{E_{Q}\left[\int u(x) \tilde{X}(\cdot, d x) \mid \mathscr{G}\right]+\alpha_{\min }^{\mathscr{G}}(Q)\right\}
$$

Remark 5.6. If we restrict the conditional preference relation to $\mathcal{L}$, then 5.10 and 5.11 reduce to:

$$
U(X)=-\rho(u(X))=\operatorname{essinf}_{Q \in \mathcal{M}_{1, f}(P \mid \mathscr{G})}\left\{E_{Q}[u(X) \mid \mathscr{G}]+\alpha_{m i n}^{\mathscr{G}}(Q)\right\} .
$$

Moreover, if the preference order satisfies the continuity assumption 5.12 and $u$ is $P$ almost surely unbounded, then we get:

$$
U(X)=\underset{Q \in \mathcal{M}_{1}(\mathscr{G} \mid P)}{\operatorname{essinf}}\left\{E_{Q}[u(X) \mid \mathscr{G}]+\alpha_{\min }^{\mathscr{G}}(Q)\right\}
$$

Proof. For the existence of a general numerical representation $\tilde{U}$, we use a conditional version of the arguments given in [Föllmer and Schied, 2004]: the preference relation $\succcurlyeq$ restricted to $\mathcal{M}_{1, c}(\mathscr{G})$ fulfills the independence axiom $\mathrm{A}-\mathrm{V}^{\prime \prime}$ hence, according Theorem 5.1 , there exists a function $u: \mathbb{R} \times \Omega \rightarrow \mathbb{R}$ monotone which is increasing, continuous in the first component, and $\mathscr{G}$-measurable in the second one such that

$$
\tilde{u}(\mu)(\omega)=\int u(\omega, x) \mu(\omega, d x), \quad \mu \in \mathcal{M}_{1, c}(\mathscr{G}),
$$

is a numerical representation of $\succcurlyeq$ restricted to $\mathcal{M}_{1, c}(\mathscr{G})$.

Further, we extend $\tilde{u}$ to a numerical representation $\tilde{U}$ of $\succcurlyeq$ on $\tilde{\mathcal{X}}$. For this, take $\tilde{X} \in \tilde{\mathcal{X}}$ and consider $c>0$ such that $\tilde{X}(\omega, \cdot) \in \mathcal{M}_{1,[-c, c]}$ for almost all $\omega \in \Omega$. Hence $\tilde{u}\left(\delta_{c}\right) \geq \tilde{u}(\tilde{X}(\omega, \cdot)) \geq \tilde{u}\left(\delta_{-c}\right)$. The monotonicity axiom A-IV yields then

$$
\delta_{-c} \preccurlyeq \tilde{X} \preccurlyeq \delta_{c} \quad \text { and } \quad \delta_{-c} \prec \delta_{c} \text {. }
$$

Using the same arguments as in the demonstration of (ii) of Lemma 5.3, we get the existence of a $\mathscr{G}$-measurable function $\alpha$ with values in $[0,1]$ such that $\tilde{X} \sim \alpha \delta_{-c}+$ $(1-\alpha) \delta_{c}$ with the exception that the uniqueness of such a function $\alpha$ is given by the monotonicity axiom A-IV. The only possible choice for $\tilde{U}(\tilde{X})$ is

$$
\tilde{U}(\tilde{X})=\alpha \tilde{u}\left(\delta_{-c}\right)+(1-\alpha) \tilde{u}\left(\delta_{c}\right) .
$$

It is plain to check that $\tilde{U}(\tilde{X})$ does not depends on the choice of $c$ by taking $c^{\prime}>c$ and expressing $\delta_{c}, \delta_{-c}$ and $\tilde{X}$ as convex combinations of $\delta_{c^{\prime}}$ and $\delta_{-c^{\prime}}$.

We have now a numerical representation of $\succcurlyeq$ on $\tilde{\mathcal{X}}$ and in particular, the embedding of $\mathcal{L}$ into $\tilde{\mathcal{X}}$ delivers the existence of a numerical representation $U$ of the restriction of 
$\succcurlyeq$ to $\mathcal{L}$ given by

$$
U(X) \equiv \tilde{U}\left(\delta_{X}\right) \quad \text { for any } X \in \mathcal{L} .
$$

Here also, a conditional positive affine transformation $u_{a, b}=a u+b$ yields the relation $U_{a, b}=a U+b$.

Applying then the results of Proposition 5.7 stating the existence of a conditional cash additive risk measure $\rho: \mathcal{L} \rightarrow \mathbb{L}^{\infty}(\Omega, \mathscr{G}, P)$ such that

$$
U(X)=-\rho(u(X))
$$

ends the demonstration for the representation (5.10) which together with Proposition 5.8 implies (5.11).

We show now that under condition (5.12), the conditional convex risk measure $\rho$ is continuous from below. Take $X, X_{n}$ in $\mathcal{L}$ such that $X_{n} \nearrow X$ pointwise, and let us show that $\rho\left(X_{n}\right) \searrow \rho(X)$. By the translation invariance and due to the unboundedness of $u P$-almost surely, we can suppose that $X_{n}$ and $X$ are in the range of $u$. Defining $\bar{X}_{n}=u^{-1}\left(\cdot, X_{n}\right), \bar{X}=u^{-1}(\cdot, X)$, as in Proposition 5.7, the condition (5.12) holds for $\bar{X}, \bar{X}_{n}$ and $\bar{Y}_{\varepsilon}=u^{-1}(\cdot, X-\varepsilon)$ for any $\varepsilon>0$. Thus, for any $\varepsilon>0$, there exists $n_{0} \in \mathbb{N}$ such that for any $n \geq n_{0}$ holds

$$
\rho(X) \leq \rho\left(X_{n}\right)=-U\left(\bar{X}_{n}\right) \leq-U\left(\bar{Y}_{\varepsilon}\right)=\rho(X-\varepsilon)=\rho(X)+\varepsilon
$$

This means that $\rho\left(X_{n}\right) \searrow \rho(X)$ showing that $\rho$ is continuous from below. We can then apply the second part of the Proposition 5.8 to get the second point of the theorem and end the proof.

The following proposition formulates the special case of the general representation 5.10 when the conditional preference relation is restricted to the space $\mathcal{L}$ of random variables. This proof is crucial step in the demonstration of the preceding theorem.

Proposition 5.7. Given $u$ of (5.16) and the conditional numerical representation $U$ on $\mathcal{L}$ given by (5.18), there exists a conditional cash additive risk measure $\rho: \mathcal{L} \rightarrow$ $\mathbb{L}^{\infty}(\Omega, \mathscr{G}, P)$ such that

$$
U(X)=-\rho(u(\cdot, X)), \quad X \in \mathcal{L} .
$$

We use here the same technique as for the Corollary 3.20 stating the same result for the unconditional case.

Proof. For $u$ coming from (5.16), we define the conditional convex set

$$
(A, B)=\{X \in \mathcal{L} \mid A<\operatorname{essinf} X \leq \operatorname{ess} \sup X<B\}=u(\cdot, \mathcal{L})
$$

where $A(\cdot)=\inf _{x \in \mathbb{R}} u(\cdot, x) \in \mathbb{L}^{0}(\Omega, \mathscr{G}, P)$ and $B(\cdot)=\sup _{x \in \mathbb{R}} u(\cdot, x) \in \mathbb{L}^{0}(\Omega, \mathscr{G}, P)$. Without lost of generality, we can assume that $A<-1<+1<B P$-almost surely 


\section{Conditional Robust Representations}

because we can take any positive affine transformation of $u$ to represent the conditional preference relation.

In reason of the strict monotonicity and continuity, $u$ states a bijection between $\mathcal{L}$ and $(A, B)$. We therefore define the functional

$$
\begin{aligned}
\hat{\rho}:(A, B) & \longrightarrow \mathbb{L}^{\infty}(\Omega, \mathscr{G}, P) \\
X & \longmapsto \hat{\rho}(X):=-U\left(u^{-1}(\cdot, X)\right)
\end{aligned}
$$

whereby $u^{-1}(\cdot, x)$ denote the $\omega$-wise inverse of the increasing continuous function $x \mapsto$ $u(\cdot, x)$. This functional is by definition clearly monotone on $(A, B)$ from the monotonicity of $U$ and $u^{-1}$. Furthermore, it is straightforward to check from the risk aversion axiom A-III that $\hat{\rho}$ is conditionally quasiconvex, that is

$$
\hat{\rho}(\alpha X+(1-\alpha) Y) \leq \operatorname{ess} \sup \{\hat{\rho}(X), \hat{\rho}(Y)\},
$$

for any $X, Y \in(A, B)$ and $\alpha \in \mathbb{L}^{\infty}(\Omega, \mathscr{G}, P)$ with $0 \leq \alpha \leq 1$.

We have to check now whether $\hat{\rho}$ can be extended to a conditional cash additive risk measure $\rho: \mathcal{L} \rightarrow \mathbb{L}^{\infty}(\Omega, \mathscr{F}, P)$. To do so, take $X \in(A, B)$, a $\mathscr{G}$-measurable random variable $\lambda$ such that $0<\lambda<1$ and $X / \lambda \in(A, B)$ as well as $-\hat{\rho}(X) / \lambda \in(A, B)$. Finally, take $m \in \mathbb{L}^{\infty}(\Omega, \mathscr{G}, P)$ such that $(1-\lambda) A<m<(1-\lambda) B$. For $X_{0}=u^{-1}(\cdot, X / \lambda)$ we define

$$
\tilde{Z}_{m}=\lambda \delta_{X_{0}}+(1-\lambda) \delta_{u^{-1}(\cdot, m /(1-\lambda))}
$$

and

$$
Z_{m}=u^{-1}\left(\cdot, \lambda u\left(\cdot, X_{0}\right)+(1-\lambda) u^{-1}\left(\cdot, u\left(\cdot, \frac{m}{1-\lambda}\right)\right)\right)=u^{-1}(\cdot, X+m) .
$$

For any fixed $\omega \in \Omega$, holds

$$
\tilde{U}\left(\tilde{Z}_{m}(\omega)\right)=\lambda u\left(\cdot, X_{0}(\omega)\right)+m=\tilde{U}\left(\delta_{Z_{m}(\omega)}\right),
$$

therefore, the monotonicity axiom A-IV yields $\tilde{Z}_{m} \sim \delta_{Z_{m}}$, hence

$$
\tilde{U}\left(\tilde{Z}_{m}\right)=U\left(Z_{m}\right)=\hat{\rho}(X+m) .
$$

Due to the definition of $\tilde{\mathcal{X}}$, there exists some measures $\mu_{1}, \mu_{2} \in \mathcal{M}_{1, c}(\mathscr{G})$ with $\mu_{1} \succcurlyeq$ $\delta_{X_{0}(\omega)} \succcurlyeq \mu_{2}$ for all $\omega \in \Omega$, therefore

$$
\begin{aligned}
\lambda \mu_{1}+(1-\lambda) \delta_{u^{-1}(m /(1-\lambda))} & \succcurlyeq \lambda \delta_{X_{0}(\omega)}+(1-\lambda) \delta_{u^{-1}(m /(1-\lambda))} \\
& \succcurlyeq \lambda \mu_{1}+(1-\lambda) \delta_{u^{-1}(m /(1-\lambda))},
\end{aligned}
$$


and the monotonicity axiom A-IV yields

$\lambda \mu_{1}+(1-\lambda) \delta_{u^{-1}(m /(1-\lambda))} \succcurlyeq \lambda \delta_{X_{0}}+(1-\lambda) \delta_{u^{-1}(m /(1-\lambda))} \succcurlyeq \lambda \mu_{1}+(1-\lambda) \delta_{u^{-1}(m /(1-\lambda))}$.

From the continuity axiom, we can find some $\mathscr{G}$-measurable function $\alpha$ with values in $[0,1]$ such that

$$
\begin{aligned}
& \lambda \delta_{X_{0}}+(1-\lambda) \delta_{u^{-1}(m /(1-\lambda))} \\
& \sim \alpha\left(\lambda \mu_{1}+(1-\lambda) \delta_{u^{-1}(m /(1-\lambda))}\right)+(1-\alpha)\left(\lambda \mu_{2}+(1-\lambda) \delta_{u^{-1}(m /(1-\lambda))}\right) \\
& =\lambda \nu+(1-\lambda) \delta_{u^{-1}(m /(1-\lambda))},
\end{aligned}
$$

where $\nu=\alpha \mu_{1}+(1-\alpha) \mu_{2}$. The weak certainty independence axiom implies

$$
\lambda \delta_{X_{0}}+(1-\lambda) \delta_{0} \sim \lambda \nu+(1-\lambda) \delta_{0}
$$

and this together with $(5.20)$ yields:

$$
\begin{array}{r}
-\hat{\rho}(X+m)=\tilde{U}\left(\lambda \nu+(1-\lambda) \delta_{u^{-1}(m /(1-\lambda))}\right)=\tilde{u}(\lambda \nu)+(1-\lambda) \tilde{u}\left(\delta_{u^{-1}(m /(1-\lambda))}\right) \\
=\tilde{u}(\lambda \nu)+(1-\lambda) u(0)+m=\tilde{U}\left(\lambda \nu+(1-\lambda) \delta_{0}\right)+m=\tilde{U}\left(\tilde{Z}_{0}\right)+m \\
=-\hat{\rho}(X+u(0))+m=-\hat{\rho}(X)+m .
\end{array}
$$

We deduce then

$$
\hat{\rho}(X+m)=\hat{\rho}(X)-m
$$

for any $X \in(A, B)$ and $m \in \mathbb{L}^{\infty}(\Omega, \mathscr{G}, P)$ such that $X / \lambda \in(A, B)$ and $A(1-\lambda)<m<$ $(1-\lambda) B$. This means that $\hat{\rho}$ is $P$-almost surely Gateau differentiable at any point in $(A, B)$ in direction of 1 , and this derivative is equal to -1 . We therefore have $P$-almost surely

$$
\hat{\rho}(X+m)=\hat{\rho}(X)+\int_{0}^{m}-1 d s=\hat{\rho}(X)-m,
$$

for any $X \in(A, B)$ and $m \in \mathbb{L}^{\infty}(\Omega, \mathscr{G}, P)$ such that $X+m \in(A, B)$. We now extend $\hat{\rho}$ to $\mathcal{L}$ by

$$
\rho(X)=\operatorname{essinf}\left\{\hat{\rho}(Y)-m \mid X \geq Y+m, Y, m \in(A, B) \times \mathbb{L}^{\infty}(\Omega, \mathscr{G}, P)\right\}, \quad X \in \mathcal{L},
$$

This functional is well defined with values in $\mathbb{L}^{\infty}(\Omega, \mathscr{G}, P)$ and per definition translation invariant. It furthermore inherits clearly from the properties of monotonicity and quasiconvexity of $\hat{\rho}$. It coincides also with $\hat{\rho}$ on $(A, B)$. Indeed, suppose that $X \in(A, B)$, direct computations from the monotonicity shows that if $Y+m \notin(A, B)$, it can not be 


\section{Conditional Robust Representations}

optimal in the essinf sense definition of $\hat{\rho}$, hence

$$
\begin{aligned}
& \rho(X) \\
& \quad=\operatorname{essinf}\left\{\hat{\rho}(Y)-m \mid X \geq Y+m, Y, Y+m \in(A, B) \text { and } m \in \mathbb{L}^{\infty}(\Omega, \mathscr{G}, P)\right\} \\
& =\operatorname{essinf}\left\{\hat{\rho}(Y+m) \mid X \geq Y+m, Y, Y+m \in(A, B) \text { and } m \in \mathbb{L}^{\infty}(\Omega, \mathscr{G}, P)\right\} \\
& =\operatorname{essinf}\left\{\hat{\rho}\left(Y^{\prime}\right) \mid X \geq Y^{\prime} \text { with } Y \in(A, B)\right\} \\
& =\hat{\rho}(X),
\end{aligned}
$$

where we used in the third line the translation invariance of $\hat{\rho}$ in the strip $(A, B)$.

We are left to show that $\rho$ is conditionally convex. Alike in the static case, this is a consequence of the quasiconvexity together with the cash invariance. To be self contained, we explicit the proof in our context. Take $X, Y \in \mathcal{L}$ and $\alpha \in \mathbb{L}^{\infty}(\Omega, \mathscr{G}, P)$ with $0 \leq \alpha \leq 1$, then $\alpha \rho(X)+(1-\alpha) \rho(Y) \in \mathbb{L}^{\infty}(\Omega, \mathscr{G}, P)$, therefore, using cash invariance and quasiconvexity

$$
\begin{aligned}
\rho(\alpha X+(1-\alpha) Y) & -\alpha \rho(X)-(1-\alpha) \rho(Y) \\
= & \rho(\alpha X+(1-\alpha) Y+\alpha \rho(X)+(1-\alpha) \rho(Y)) \\
= & \rho(\alpha(X+\rho(X))+(1-\alpha)(Y+\rho(Y))) \\
\leq & \operatorname{essinf}\{\rho(X+\rho(X)), \rho(Y+\rho(Y))\} \\
& =\operatorname{essinf}\{\rho(X)-\rho(X), \rho(Y)-\rho(Y)\}=0,
\end{aligned}
$$

and therefore

$$
\rho(\alpha X+(1-\lambda) Y) \leq \alpha \rho(X)+(1-\alpha) \rho(Y),
$$

ending the proof that $\rho$ is a conditional cash additive risk measure.

The next proposition gives a representation of conditional convex risk measures in terms of penalty functions in the setting of positive finitely additive set functions. Such a representation is classic in the case where a probability measure $P$ is given on $(\Omega, \mathscr{F})$ and with some continuity from below, see e.g. [Detlefsen and Scandolo, 2005, Föllmer and Penner, 2006], which is not a priori our case.

A possible approach could have been an adaptation of the proof in Detlefsen and Scandolo [2005], projecting the conditional risk measure on the trivial $\sigma$-algebra to reduce the problem to the unconditional case. Instead of this, we profit of the opportunity to give a direct proof of theorem 4.5 in [Föllmer and Schied, 2004] using the methods of conditional convex analysis introduced by Filipovic, Kupper, And Vogelpoth [2009].

Proposition 5.8. Let $\rho: \mathcal{L} \longrightarrow \mathbb{L}^{\infty}(\Omega, \mathscr{G}, P)$ be a conditional convex risk measure. 
There exists then a penalty function $\alpha_{\text {min }}^{\mathscr{G}}: \mathcal{M}_{1, f}(P \mid \mathscr{G}) \rightarrow \overline{\mathbb{L}}_{+}^{0}(\Omega, \mathscr{G}, P)$ such that

$$
\rho(X)=\operatorname{ess}_{Q \in \mathcal{M}_{1, f}(P \mid \mathscr{G})}\left\{E_{Q}[-X \mid \mathscr{G}]-\alpha_{\text {min }}^{\mathscr{G}}(Q)\right\}, \quad X \in \mathcal{L},
$$

where $\alpha_{\min }^{\mathscr{G}}$ is explicitly given by

$$
\alpha_{\min }^{\mathscr{G}}(Q)=\underset{X \in \mathcal{A}_{\rho}}{\operatorname{ess} \sup _{Q}} E_{Q}[-X \mid \mathscr{G}], \quad Q \in \mathcal{M}_{1, f}(P \mid \mathscr{G}),
$$

and

$$
\mathcal{A}_{\rho}=\{X \in \mathcal{L} \mid \rho(X) \leq 0 \quad P \text {-almost surely }\} .
$$

Moreover, if $\rho$ is continuous from below, i.e. $X_{n}(\omega) \nearrow X(\omega)$ for all $\omega \in \Omega$ implies $\rho\left(X_{n}\right) \searrow \rho(X) P$-a.s., then $\alpha_{\text {min }}^{\mathscr{G}}$ is concentrated on $\mathcal{M}_{1}(P, \mathscr{G})$, i.e.:

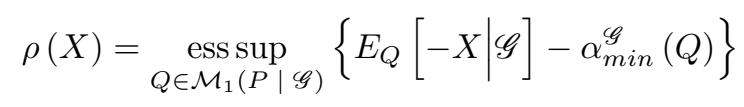

Before we go on the proof, we need the following Lemma guarantying the existence of conditional expectation with respect to $\mathscr{G}$ for additive set functions ${ }^{1}$ equal to $P$ on $\mathscr{G}$.

Lemma 5.9. For any $Q \in \mathcal{M}_{1, f}(P \mid \mathscr{G})$, there exists a conditional expectation with respect to $\mathscr{G}$ on $\mathcal{L}$, that is for any $X \in \mathcal{L}$ there exists a random variable $E_{Q}[X \mid \mathscr{G}] \in$ $\mathbb{L}^{\infty}(\Omega, \mathscr{G}, P)$ such that

$$
E_{Q}\left[X \nVdash_{A}\right]=E_{Q}\left[E_{Q}[X \mid \mathscr{G}] \nVdash_{A}\right],
$$

for any $A \in \mathscr{G}$ and this random variable is unique $P$-almost surely

Proof. The a-priori difficulty here is that $Q$ is only a finitely additive set function, but on $\mathscr{G}$ it is a probability measure equal to $P$.

Consider some positive $X \in \mathcal{L}$ and define $Q_{X}[A]=E_{Q}\left[X \nVdash_{A}\right]$ for any $A \in \mathcal{G}$. It is plain to check that $Q_{X}$ is a positive finitely additive set function on $\mathscr{G}$ with finite total variations. It is also absolutely continuous with respect to $Q=P$ over $\mathscr{G}$. Furthermore, if we take a sequence of mutually disjoint sets $\left(A_{n}\right)_{n \in \mathbb{N}}$ in $\mathscr{G}$, we get

$$
\begin{aligned}
0 \leq Q_{X}\left[\bigcup_{k=0}^{\infty} A_{k}\right]-\sum_{k=0}^{n} Q_{X}\left[A_{k}\right] & =E_{Q}\left[X\left[\nVdash_{\left\{\bigcup_{k=0}^{\infty} A_{k}\right\}}-\nVdash_{\left\{\bigcup_{k=0}^{n} A_{k}\right\}}\right]\right] \\
& \leq\|X\|_{\infty}\left[Q\left[\bigcup_{k=0}^{\infty} A_{k}\right]-\sum_{k=0}^{n} Q\left[A_{k}\right]\right] \\
& =\|X\|_{\infty}\left[P\left[\bigcup_{k=0}^{\infty} A_{k}\right]-\sum_{k=0}^{n} P\left[A_{k}\right]\right] \\
& \underset{n \rightarrow+\infty}{\longrightarrow} 0 .
\end{aligned}
$$

${ }^{1}$ Note that is in general false that additive set functions have a conditional expectation. 


\section{Conditional Robust Representations}

Hence, $Q_{X}$ is $\sigma$-additive. We can then define $E_{Q}[X \mid \mathscr{G}]$ as the Radon-Nicodym derivative $d Q_{X} / d P$, fulfilling the required property of the proposition.

For general $X \in \mathcal{L}$, we apply separately the previous step to $X^{+}$and $X^{-}$, and sum the difference.

Proof (Proposition 5.8). For the existence of the conditional expectation $E_{Q}[\cdot \mid \mathscr{G}]$ on $\mathcal{L}$ for any $Q \in \mathcal{M}_{1, f}(P \mid \mathscr{G})$, we refer to the previous Lemma 5.9.

Clearly, the inequality

$$
\rho(X) \geq \operatorname{ess}_{Q \in \mathcal{M}_{1, f}(P \mid \mathscr{G})}\left\{E_{Q}[-X \mid \mathscr{G}]-\alpha_{\min }^{\mathscr{G}}(Q)\right\}
$$

is easy to obtain, using the definition of $\alpha_{\text {min }}^{\mathscr{G}}$, the translation invariance of $\rho$ and the fact that $Y=X+\rho(X) \in \mathcal{A}_{\rho}$.

To show the other side of the inequality, we use separation arguments in a conditional setting using the hyperplane separation Theorem 2.6 in [Filipovic et al., 2009]. To do so, consider the functional

$$
\|X\|_{\infty}^{\mathscr{G}}=\operatorname{essinf}\left\{\lambda \in \mathbb{L}^{0}(\Omega, \mathscr{G}, P)|\lambda(\omega) \geq| X(\omega) \mid \text { for all } \omega \in \Omega\right\}, \quad X \in \mathcal{L} .
$$

Notice that the inequality between $X$ and $\lambda$ is taken uniformly on the whole set $\Omega$, because no probability on $(\Omega, \mathscr{F})$ is given, but having taken the essential infimum, the norm does not as usual map into $\mathbb{R}$, but in its conditional counterpart $\mathbb{L}_{+}^{0}(\Omega, \mathscr{G}, P)$. If $\mathscr{G}$ is trivial, this correspond to the classical point wise supremum norm.

It is easy to check along the lines of [Filipovic et al., 2009, Kupper and Vogelpoth, 2008 ] that this functional is a $\mathbb{L}^{0}(\Omega, \mathscr{G}, P)$-norm for the $\mathbb{L}^{0}(\Omega, \mathscr{G}, P)$-module $\mathcal{L}$. The elements $X \in \mathcal{L}$ being uniformly bounded, it makes $\mathcal{L}$ a local convex $\mathbb{L}^{0}(\Omega, \mathscr{G}, P)$-module space $^{2}$.

We want to find some $Q_{X} \in \mathcal{M}_{1, f}(P \mid \mathscr{G})$ such that

$$
\rho(X) \leq E_{Q_{X}}[-X \mid \mathscr{G}]-\alpha_{\min }^{\mathscr{G}}\left(Q_{X}\right) .
$$

Up to a translation, we can assume that $\rho(X)=0$. Therefore, $X$ is not contained in the non empty $\mathbb{L}^{0}(\Omega, \mathscr{G}, P)$-convex set

$$
\mathcal{B}=\{Y \in \mathcal{L} \mid \rho(Y)<0 \quad P \text {-almost surely }\} .
$$

As in the static case, monotony and translation invariance yields that $-\|X\|_{\infty}^{\mathscr{G}} \leq$ $\rho(X) \leq\|X\|_{\infty}^{\mathscr{G}}$ implying the standard Lipschitz continuity with respect to the $\|\cdot\|_{\infty}^{\mathscr{G}}$ norm, that is

$$
|\rho(X)-\rho(Y)| \leq\|X-Y\|_{\infty}^{\mathscr{G}} \quad P \text {-almost surely for all } X, Y \in \mathcal{L} .
$$

${ }^{2}$ For further informations and properties about convex $\mathbb{L}^{0}$-modules we refer to [Filipovic et al., 2009, Kupper and Vogelpoth, 2008]. 
Thus, $\mathcal{B}$ is open for the $\mathbb{L}^{0}(\Omega, \mathscr{G}, P)$-module norm. It is also non empty, otherwise $\rho$ would be the trivial risk measure, and for any $A \in \mathscr{G}$ with strict positive probability, we have $1_{A} \mathcal{B} \cap 1_{A}\{X\}=\emptyset$ because a conditional risk measure is such that $\rho\left(1_{A} X\right)=$ $1_{A} \rho(X)$ for any $A \in \mathscr{G}$. We can then apply the hyperplane separation in [Filipovic et al., 2009, Theorem 2.6] to get a non zero continuous $\mathbb{L}^{0}(\Omega, \mathscr{G}, P)$-linear functional $l: \mathcal{L} \rightarrow \mathbb{L}^{0}(\Omega, \mathscr{G}, P)$ such that

$$
l(X) \leq \underset{Y \in \mathcal{B}}{\operatorname{essinf}} l(Y)
$$

Monotonicity and cash-invariance of $\rho$ implies that for any positive $Y \in \mathcal{L}$ and strictly positive $\lambda \in \mathbb{L}^{\infty}(\Omega, \mathscr{G}, P), 1+\lambda Y \in \mathcal{B}$. Hence

$$
l(X) \leq l(1)+\lambda l(Y)
$$

showing that $l \geq 0$ for any positive $Y$. Because $l$ is not identically negative, there exists some $Y \in \mathcal{L}$ uniformly bounded by say $1 / 2$ such that $l(Y)>0$. Hence, positivity of $l$ over $\mathcal{L}_{+}$yields $l\left(Y^{+}\right)>0$ and $l\left(1-Y^{-}\right) \geq 0$ and therefore, $l(1)=l\left(Y^{+}\right)+l\left(1-Y^{-}\right)>$ 0 . Let us show now that

$$
\frac{l(Y)}{l(1)}=E_{Q_{X}}[Y \mid \mathscr{G}]
$$

for some $Q_{X} \in \mathcal{M}_{1, f}(P \mid \mathscr{G})$. To this aim, consider $\bar{l}=E_{P}[l / l(1)]$ which is a real linear functional on $\mathcal{L}$. By [Föllmer and Schied, 2004, Theorem A.50], there exists then $Q_{X} \in \mathcal{M}_{1, f}$ such that $\bar{l}(X)=E_{Q_{X}}[X]$. It is trivial to check that $Q_{X}=P$ over $\mathscr{G}$, by considering some $X=1_{A}$ for $A \in \mathscr{G}$, and by a simple contradiction argument that 5.31 holds. We finish by seeing that $\mathcal{B} \subset \mathcal{A}_{\rho}$ such that

$$
\alpha_{\min }^{\mathscr{G}}\left(Q_{X}\right)=\underset{Y \in \mathcal{A}_{\rho}}{\operatorname{ess} \sup _{X}} E_{Q_{X}}[Y \mid \mathscr{G}] \geq \underset{Y \in \mathcal{B}}{\operatorname{esssup}} E_{Q_{X}}[Y \mid \mathscr{G}]=-\frac{b}{l(1)} .
$$

But on the other hand, $Y+\varepsilon \in \mathcal{B}$ for any $Y \in \mathcal{A}_{\rho}$ and $\varepsilon>0$. This shows that $\alpha_{\min }^{\mathscr{G}}=-b / l(1)$. It follows

$$
E_{Q_{X}}[-X]-\alpha_{\min }^{\mathscr{G}}\left(Q_{X}\right)=\frac{1}{l(1)}(b-l(X)) \geq 0=\rho(X)=0 .
$$

Ending the proof of 5.21 .

A one to one adaptation of [Föllmer and Schied, 2004, Lemma 4.22 and Proposition 4.21 , delivers the second part of the proposition and the corresponding equality 5.24 , ending the proof.

\subsection{Distributional Risk, Model Risk}

Our general robust representation of conditional preference relation separates two crucial components: An attitude toward risk specified by the monotone continuous function $u$ which does not depend on the probability model, and an assessment of model uncertainty 


\section{Conditional Robust Representations}

in terms of the risk measure $\rho$. It is now natural to ask which additional assumptions yield a numerical representation which focus either on the attitude toward risk without any concern for the model uncertainty, or exclusively on model uncertainty, i.e., with a risk neutral attitude described by an affine function $u$.

Let us first characterise the case where no model uncertainty is taken into account. The key point is that the uncertainty aversion axiom A-III is sharpened to a conditional form of uncertainty indifference.

Proposition 5.10 (Conditional Savage Representation). For a preference order $\succcurlyeq^{\mathscr{G}}$ fulfilling the axioms of theorem 5.5, the following two assertion are equivalent:

- The conditional preference relation fulfills the stronger axiom

A-III' $\mathscr{G}$-Consistent Model Risk Indifference: From $\tilde{X}, \tilde{Y} \in \tilde{\mathcal{X}}$ with $\tilde{X} \sim^{\mathscr{G}} \tilde{Y}$, follows

$$
\alpha \tilde{X}+(1-\alpha) \tilde{Y} \sim^{\mathscr{G}} \tilde{X},
$$

for all $\mathscr{G}$-measurable function $\alpha$ with $0 \leq \alpha \leq 1$.

- The corresponding numerical representation from Theorem 5.5 is a Conditional Savage Representation, that is, there exists a finitely additive set function $Q \in$ $\mathcal{M}_{1, f}(P \mid \mathscr{G})$ such that

$$
\tilde{U}(\tilde{X})=E_{Q}\left[\int u(\cdot, x) \tilde{X}(\cdot, \omega) \mid \mathscr{G}\right], \quad \tilde{X} \in \tilde{\mathcal{X}} .
$$

In particular, the restriction of $\succcurlyeq^{\mathscr{G}}$ admits the numerical representation

$$
U(X)=E_{Q}[u(X) \mid \mathscr{G}], \quad X \in \mathcal{L}
$$

If, moreover, the preference fulfills the continuity property 5.12, the finitely additive set function $Q$ is in fact a probability measure.

Remark 5.11. The theorem shows that uncertainty independence - a restriction under which the so called Elsberg paradox continues to be in force - is equivalent to the classical form of a conditional utility functional which is widely (over)used in financial mathematics.

Remark 5.12. The unconditional version of the Savage representation was obtained by SAVAGE [1972], but only on the level of random variables. On the same level, a conditional version of the representation has been given by SkIADAS [1997A], but there, as discussed above, the axiomatic approach is formulated in terms of a family of preferences.

Proof. The representation form (5.34) is linear in $\tilde{X}$, and this clearly implies axiom A-III'. 
Conversely, suppose that axiom A-III' holds. It implies the following property for the conditional cash additive risk measure

$$
\rho(\alpha X+(1-\alpha) Y)=\alpha \rho(X)+(1-\alpha) \rho(Y),
$$

for $X, Y \in \mathcal{L}$ such that $X \sim Y$ and for any $\mathscr{G}$-measurable function $\alpha$ assuming values in $[0,1]$. Because of the translation invariance, it follows as in the proof of Proposition (5.7) that relation 5.36 holds for any $X, Y$, even without assuming equivalence.

Setting $\alpha=1 / 2$ and $Y=0$ shows that $\rho(X / 2)=\rho(X) / 2$. Now replacing $X$ by $X+Y$ yields

$$
\rho(X+Y)=2 \rho\left(\frac{X+Y}{2}\right)=\rho(X)+\rho(Y)
$$

Define the functional $Q: \mathscr{F} \rightarrow[0,1]$ by

$$
Q[A]:=-E_{P}\left[\rho\left(1_{A}\right)\right], \quad A \in \mathscr{F} .
$$

The functional is well defined and takes value between 0 and 1 because of the monotonicity of $\rho$ and the fact that $\rho(1)=-1$. Moreover, $Q[\emptyset]=0$ and $Q[\Omega]=1$. For two disjoint sets $A, B \in \mathscr{F}$ hold

$$
\begin{aligned}
Q[A \cup B]=-E_{P}\left[\rho\left(1_{A \cup B}\right)\right]=-E_{P}\left[\rho\left(1_{A}+1_{B}\right)\right] \\
\quad=-E_{P}\left[\rho\left(1_{A}\right)+\rho\left(1_{B}\right)\right]=Q[A]+Q[B] .
\end{aligned}
$$

Thus $Q$ is a finitely additive set function with values in $[0,1]$ and normalized to $Q[\Omega]=1$. Moreover, it is trivial to check that $Q=P$ over $\mathscr{G}$.

Hence the linear functional $E_{Q}[\cdot]$ over $\mathcal{L}$ is well defined, and so is the corresponding conditional expectation $E_{Q}[\cdot \mid \mathscr{G}]$; see Proposition 5.9. We have to show that $\rho=-E_{Q}[\cdot \mid \mathscr{G}]$. Note that $\rho_{0}=E_{P}[\rho(\cdot)]$ is an unconditional convex risk measure, hence Lipschitz continuous. The relation $\rho(X+Y)=\rho(X)+\rho(Y)$ coupled with this continuity shows that $-\rho_{0}$ is a continuous linear functional on $\mathcal{L}$, and so it can be written as $E_{Q^{\prime}}[\cdot]$ for a finitely additive set function $Q^{\prime} \in \mathcal{M}_{1, f}$. In view of definition (5.37), it is clear that $Q^{\prime}=Q$ over $\mathscr{F}$. Suppose now that there exists $X \in \mathcal{L}$ such that $\rho(X) \neq-E_{Q}[X \mid \mathscr{G}]$. Consider $A=\left\{\rho(X)<-E_{Q}[X \mid \mathscr{G}]\right\}$. Taking the expectation under $Q$ for the random variable $X 1_{A}$ we get

$$
E_{Q}\left[-X 1_{A}\right]=-\rho_{0}\left(X 1_{A}\right)=E_{P}\left[-\rho\left(X 1_{A}\right)\right]>E_{P}\left[E_{Q}\left[X 1_{A} \mid \mathscr{G}\right]\right]=E_{Q}\left[X 1_{A}\right],
$$

which implies $P[A]=0$. The same argument applied to the converse inequality shows that $\rho(X)=-E_{Q}[X \mid \mathscr{G}] P$-almost surely.

We suppose now that the preference relation fulfills the continuity property 5.12 . Then the conditional risk measure $\rho$ is continuous from above. Because $\mathcal{L}$ is a lattice, we can use the theorem of Daniel-Stone (see for instance [Föllmer and Schied, 2004, 


\section{Conditional Robust Representations}

theorem A.48]) to conclude that the continuous linear functional $\rho_{0}(\cdot)=-E_{P}[\rho(\cdot)]$ is continuous from above, and this means that $Q$ is indeed $\sigma$-additive.

Let us now look at the other end of the spectrum, i.e., we want to characterize the case where the numerical representation reduces to a pure risk measure. A trivial way to do so is to assume that the preferences restricted to the lotteries are risk neutral as stated in the following proposition:

Proposition 5.13. Given a preference relation $\succcurlyeq^{\mathscr{G}}$ fulfilling the same assumptions as in theorem 5.5, the following two assertions are equivalent:

- The preference relation fulfills the following risk neutral axiom:

\section{A-VII Distributional Risk Neutrality:}

$$
\tilde{X} \sim^{\mathscr{G}} \delta_{M(\tilde{X})} \quad \text { for any } \tilde{X} \in \tilde{\mathcal{X}}
$$

where $M(\tilde{X})(\omega)=\int x \tilde{X}(\omega, d x)$

- The corresponding numerical representation is a pure risk measure, i.e.,

$$
\tilde{U}(\tilde{X})=-\rho\left(\int x \tilde{X}(\cdot, d x)\right) \quad \text { for any } \tilde{X} \in \tilde{\mathcal{X}}
$$

and in particular with restriction to $\mathcal{L}$ :

$$
U(X)=-\rho(X) \quad \text { for any } X \in \mathcal{L} .
$$

The quantity $M(\tilde{X})(\omega)=\int x \tilde{X}(\omega, d x)$ is sometimes called the fair price of the lottery $\tilde{X}(\omega, \cdot)$. The proof of this proposition is straightforward, as the definition of risk neutrality is a direct translation of the fact that $u$ is affine. However, this assumption involves an integration and is not stated directly in terms of the lotteries.

In order to formulate an alternative condition, we lift the translation invariance of monetary measures to the level of preference relations. Under the additional assumption that preferences are strictly convex, this yields a complete characterization of pure risk measures. Otherwise, the translation invariance case also includes in addition to pure risk measures a combination of coherent risk measure with exponential utility.

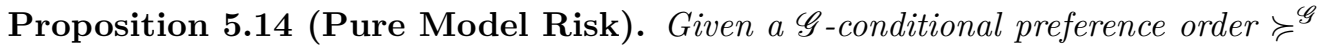
which fulfills the same assumptions as in theorem 5.5, the following two assertions are equivalent:

- The preference relation fulfills the following translation axiom:

A-VIII Translation invariance: For all $\tilde{X}, \tilde{Y} \in \tilde{\mathcal{X}}$,

$$
\tilde{X} \sim^{\mathscr{G}} \tilde{Y} \quad \Longrightarrow \quad T_{m} \tilde{X} \sim^{\mathscr{G}} T_{m} \tilde{Y} \quad \text { for all } m \in \mathbb{L}^{\infty}(\Omega, \mathscr{F}, \mathbb{P})
$$


where $T_{m}$ is the translation operator ${ }^{3}$ of a distribution of $m$.

- The corresponding numerical representation is one of the following two forms:

(i) $\tilde{U}(\tilde{X})=-\rho\left(\int x \tilde{X}(\cdot, d x)\right)$ for any $X \in \tilde{X}$. In particular:

$$
U(X)=-\rho(X), \quad X \in \mathcal{L} .
$$

(ii) $\tilde{U}(\tilde{X})=-\rho^{c o h}\left(\int\left(e^{a x}-\frac{1}{a}\right) \tilde{X}(\cdot, d x)\right)$ where $\rho^{\text {coh }}$ is a coherent risk measure, in particular:

$$
U(X)=-\rho^{c o h}\left(e^{a X}-1 / a\right), \quad X \in \mathcal{L} .
$$

If the preferences are strictly convex as stated in axiom A-III, then the second case (ii) is excluded and the risk measure $\rho$ in (i) is strictly convex.

Proof. A simple computation shows that both (i) and (ii) imply translation invariance of the preferences. Suppose now that the translation axiom holds. Due to Theorem 5.5 the preferences over $\tilde{\mathcal{X}}$ are given by

$$
\tilde{U}(X)=-\rho\left(\int u(x) \tilde{X}(\cdot, d x)\right),
$$

for a $\mathscr{G}$-conditional cash additive risk measure $\rho$ and a uniformly bounded, monotone and continuous $\mathscr{G}$-measurable function $u$. This representation implies in particular that $\mu \sim \delta_{c(\mu)}$ for any $\mu \in \mathcal{M}_{1, c}(\mathscr{G})$ where

$$
c(\mu)=u^{-1}\left(\int u(x) \mu(d x)\right),
$$

is the so called certainty equivalent. The translation invariance axiom applied to $\mu \sim$ $\delta_{c(\mu)}$ yields

$$
u(c(\mu)+m)=\int u(x+m) \mu(d x),
$$

for any $m \in \mathbb{R}$.

We show first that $u$ is $C^{1} P$-almost surely. To this end, note with $N$ the $P$-null set such that $u(\omega, x)$ is continuous for any $\omega \in \Omega \backslash N$ and consider as distribution the uniform one on $[0,1]$, i.e., $\mu=U n i f[0,1]$. In that case, equation 5.44 yields

$$
u(\omega, c(\mu)+m)=\int_{m}^{m+1} u(\omega, x) d x .
$$

\footnotetext{
${ }^{3}$ For a distribution $\mu \in \mathcal{M}_{1, c}(\mathscr{G})$, the translation operator for $m \in \mathbb{L}^{\infty}(\Omega, \mathscr{G}, P)$ is the convolution $T_{m} \mu=\delta_{m} \otimes \mu$ which translate the support of $\mu$ by $m$.
} 


\section{Conditional Robust Representations}

for any $m \in \mathbb{R}$. Differentiating the right hand side by $m$ yields

$$
u^{\prime}(\omega, c(\mu)+m)=u(\omega, m+1)-u(\omega, m),
$$

and therefore $u^{\prime}(\omega, m)=u(\omega, 1+m-c(\mu))-u(\omega, m)$ showing that $u(\omega, \cdot)$ is $C^{1}$ for any $\omega \in \Omega \backslash N$.

Let us now show that $u$ is in fact either an affine or an exponential utility function. Normalize $u$ by a positive affine transformation such that $u(0)=0$ and $u^{\prime}(0)=1$. Consider for each $x, y \in \mathbb{R}$ the deterministic measures $\mu_{x, y}=\frac{1}{2}\left(\delta_{x}+\delta_{y}\right)$ and $\bar{\mu}_{x, y}=$ $\delta_{c\left(\mu_{x, y}\right)}$ where $c\left(\mu_{x, y}\right)=u^{-1}\left(\frac{1}{2}(u(x)+u(y))\right)$. It is plain to check that $\mu_{x, y} \sim \bar{\mu}_{x, y}$, hence

$$
\frac{1}{2}(u(x+m)+u(y+m))=u\left(u^{-1}\left(\frac{1}{2}(u(x)+u(y))\right)+m\right),
$$

for each $m \in \mathbb{R}$. Differentiating both sides with respect to $m$ at $m=0$ yields

$$
\frac{u^{\prime}(x)+u^{\prime}(y)}{2}=u^{\prime}\left(u^{-1}\left(\frac{u(x)+u(y)}{2}\right)\right) .
$$

Applying this formula to $x^{\prime}=u^{-1}(x)$ and $y^{\prime}=u^{-1}(y)$ and defining $f=u^{\prime} \circ u^{-1}$ yields

$$
\frac{f(x)+f(y)}{2}=f\left(\frac{x+y}{2}\right) .
$$

This formula, so called Jensen functional equation, see for instance [Aczél and Dhombres, 1989], holds for any $x, y$ in the range of $u^{-1}$ which is $\mathbb{R}$ and it implies that the continuous function $f$ is in fact affine. This yields the differential equation

$$
u^{\prime}=a u+b .
$$

Under the assumption that $u^{\prime}(0)=1$ and $u(0)=0$, we get that $b=1$ and the following candidates for $u$

1. $u(x)=x$ if $a=0$;

2. $u(x)=e^{a x}-\frac{1}{a}$ if $a \neq 0$.

In the first case, we are done. It remains then to show that if the second case occurs and $\rho$ has to be coherent. Take any random variable $X \in \mathcal{L}$ and define $Y=$ $u^{-1}(-\rho(u(X)))$. Because of the cash invariance of $\rho$, we have $X \sim Y$, hence $X+m \sim$ $Y+m$. Explicitly,

$$
\begin{aligned}
-\rho\left(e^{a(X+m)}-\frac{1}{a}\right) & =u(Y+m) \\
& =\exp \left(\ln \left(-\rho(u(X))+\frac{1}{a}\right)\right) e^{a m}-\frac{1}{a} \\
& =-\rho(u(X)) e^{a m}+\frac{e^{a m}}{a}-\frac{1}{a},
\end{aligned}
$$


hence,

$$
\rho\left(e^{a m} u(X)\right)=e^{a m} \rho(u(X)) .
$$

thus $\rho$ is a coherent risk measure. 



\section{Bibliography}

B. Acciaio, H. Föllmer, and I. Penner. Risk Assessment for Uncertain Cash Flows:Model Ambiguity, Discounting Ambiguity, and the Role of Bubblesy. Preprint, 2010.

J. Aczél and J. G. Dhombres. Functional Equations in Several Variables. Cambridge University Press, 1989.

C. Aliprantis and K. Border. Infinite Dimensional Analysis: a Hitchhiker's Guide. Springer Verlag, 2006.

F. Anscombe and R. Aumann. A Definition of Subjective Probability. Annals of Mathematical Statistics, 34:199-205, 1963.

P. Artzner, F. Delbaen, J. M. Eber, and D. Heath. Coherent Measure of Risk. Mathematical Finance, 9:203-228, 1999.

R. J. Aumann. Utility Theory without the Completeness Axiom. Econometrica, 30(3): 445-462, 1962. ISSN 00129682.

R. J. Aumann and R. Serrano. An Economic Index of Riskiness. Journal of Political Economy, 116(5):810-836, 2008.

P. Bank and F. Riedel. Optimal consumption choice with intertemporal substitution. Ann. Appl. Probab., 11(3):750-788, 2001.

A. Ben-Tal and M. Teboulle. Expected Utility, Penalty Functions and Duality in Stochastic Nonlinear Programming. Management Science, 32:1445-1466, 1986.

A. Ben-Tal and M. Teboulle. An Old-New Concept Of Convex Risk Measures: The Optimized Certainty Equivalent. Mathematical Finance, 17(3):449-476, 2007.

D. Bernoulli. Specimen theoriae novae de mensura sortis, Commentarii Academiae Scientiarum Imperialis Petropolitanae (5, 175-192, 1738). Econometrica, 22:23-36, 1954. Translated by L. Sommer.

J. Borwein. Automatic Continuity and Openness of Convex Relations. Proceedings of the American Mathematical Society, 99:49-55, 1987.

G. Bosi and G. B. Mehta. Existence of a Semicontinuous or Continuous Utility Function: a Unified Approach and an Elementary Proof. Journal of Mathematical Economics, 38(3):311-328, November 2002. 
Bibliography

D. Brown, E. De Giorgi, and M. Sim. A Satisfying Alternative to Prospect Theory. Preprint, 2009.

S. Cerreia-Vioglio, F. Maccheroni, M. Marinacci, and L. Montrucchio. Uncertainty Averse Preferences. Preprint, 2008a.

S. Cerreia-Vioglio, F. Maccheroni, M. Marinacci, and L. Montrucchio. Complete Monotone Quasiconcave Duality. Preprint, 2008b.

S. Cerreia-Vioglio, F. Maccheroni, M. Marinacci, and L. Montrucchio. Risk Measures: Rationality and Diversification. Preprint, 2010.

P. Cheridito and M. Kupper. Composition of Time-Consistent Dynamic Monetary Risk Measures in Discrete Time. Preprint, 2009a.

P. Cheridito and M. Kupper. Recursivity of Indifference Prices and TranslationInvariant Preferences. Mathematics and Financial Economics, 2:173 - 188, 2009b.

A. Cherny and D. Madan. New Measures for Performance Evaluation. Review of Financial Studies, 22:2571-2606, 2009.

J. Crouzeix. Conditions for Convex of Quasiconvex Functions. Mathematics of Operations Reserach, 5:120-125, 1980.

B. de Finetti. Sulle Stratificazioni Convesse. Annali di Matematica Pura e Applicata, 30:173-183, 1949.

G. Debreu. Representation of a Preference Ordering by a Numerical Function. Thrall, R.M., Coombs, C.H. and Davis, R.L., Editors, pages 159-165, 1954.

F. Delbaen. Coherent Utility Functions. Pretoria Lecture Notes, 2003.

F. Delbaen, S. Drapeau, and M. Kupper. A von Neumann-Morgenstern Representation Result without Weak Continuity Assumption. Preprint (ssrn), 2010.

K. Detlefsen and G. Scandolo. Conditional and Dynamic Convex Risk Measure. Finance and Stochastics, 9:539-561, 2005.

S. Drapeau and M. Kupper. Risk Preferences and their Robust Representation. Preprint (ssrn), 2010.

N. El Karoui and C. Ravanelli. Cash Sub-additive Risk Measures and Interest Rate Ambiguity. Mathematical Finance, 19:561 - 590, 2009.

D. Filipovic, M. Kupper, and N. Vogelpoth. Separation and Duality in Locally $L^{0}$ Convex Modules. Journal of Functional Analysis, 256:3996 - 4029, 2009.

P. Fishburn. Nonlinear Preference and Utility Theory. Johns Hopkins University Press, Baltimore, 1988. 
H. Föllmer and I. Penner. Convex Risk Measures and the Dynamics of their Penalty Functions. Statistics \& Decision, 24(1):61-96, 2006.

H. Föllmer and A. Schied. Convex Measures of Risk and Trading Constraint. Finance and Stochastics, 6(4):429-447, 2002.

H. Föllmer and A. Schied. Stochastic Finance. An Introduction in Discrete Time. de Gruyter Studies in Mathematik. Walter de Gruyter, Berlin, New York, 2 edition, 2004.

H. Föllmer, A. Schied, and S. Weber. Robust Preferences and Robust Portfolio Choice. Handbook of Numerical Analysis, XV, Bensoussan \& Zhang (Editors), Mathematical Modeling and Numerical Methods in Finance, art. Bensoussan \& Zhang, 2009.

D. P. Foster and S. Hart. An Operational Measure of Riskiness. Journal of Political Economy, forthcoming.

M. Frittelli and E. Rosazza Gianin. Putting order in risk measures. Journal of Banking \& Finance, 26(7):1473-1486, July 2002.

I. Gilboa and D. Schmeidler. Maximin Expected Utility with a Non-Unique Prior. Journal of Mathematical Economics, 18:141-153, 1989.

H. Greenberg and W. Pierskalla. Quasiconjugate Functions and Surrogate Duality. Cahiers du Centre d'Etude de Recherche Operationelle, 15:437-448, 1973.

D. Heath. Back to the Future. In Plenary Lecture at the First World Congress of the Bachelier Society, Paris 2000.

A. Hindy, C.-F. Huang, and D. Kreps. On Intertemporal Preferences in Continuous Time: The Case of Certainty. Journal of Mathematical Economics, 21:401-440, 1992.

IPCC. Special Report on Emissions Scenarios. Technical report, Intergovernmental Panel on Climate Change, 2000.

IPCC. Climate change 2007: Synthesis report. summary for policymakers. Technical report, Intergovernmental Panel on Climate Change, 2007.

L. E. Jones. A Competitive Model of Commodity Differentiation. Econometrica, 52(2): 507-30, March 1984.

D. Kahneman. Choices, Values, and Frames. Cambridge University Press, September 2000.

D. Kahneman and A. Tversky. Prospect Theory: An Analysis of Decision under Risk. Econometrica, 47(2):263-91, March 1979.

O. Kallenberg. Foundations of Modern Probability. Probability and its Applications (New York). Springer-Verlag, New York, second edition, 2002. 
Bibliography

J. M. Keynes. The General Theory of Employment, Interest and Money. Macmillan, London, 1936.

J. M. Keynes. General Theory of Employment. Quaterly Journal of Economics, 1937. Clarification on some critics on the Book "The General Theory of Employment, Interest and Money".

F. H. Knight. Risk, Uncertainty, and Profit. Hart, Schaffner \& Marx; Houghton Mifflin Company, Boston, MA, 1921.

D. M. Kreps and E. L. Porteus. Temporal von Neumann-Morgenstern and Induced Preferences. Journal of Economic Theory, 20(1):81-109, February 1979.

M. Kupper and N. Vogelpoth. Complete $L^{0}$-Normed Modules and Automatic Continuity of Monotone Convex Functions. Preprint, 2008.

A. Lord Turner. The Turner Review: A regulatory response to the global banking crisis. Technical report, Financial Service Authority, UK, 2009.

N. Luhmann. Modern Society Shocked by its Risks. Social Sciences Research Centre: Occasional Papers, 1996.

N. Luhmann. Risk: A Sociological Theory. Transaction Publishers, 2002.

F. Maccheroni, M. Marinacci, and A. Rustichini. Ambiguity Aversion, Robustness, and the Variational Representation of Preferences. Econometrica, 74(6):1447-1498, November 2006a.

F. Maccheroni, M. Marinacci, and A. Rustichini. Dynamic Variational Preferences. Journal of Economic Theory, 127(1):4-44, May 2006b.

F. Maccheroni, M. Marinacci, A. Rustichini, and M. Taboga. Portfolio Selection With Monotone Mean-Variance Preferences. Mathematical Finance, 19(3):487-521, 2009.

H. Markowitz. Portfolio Selection. The Journal of Finance, 7(1):77-91, 1952.

A. Mas-Colell. The Price Equilibrium Existence Problem in Topological Vector Lattices. Econometrica, 54(5):1039-53, September 1986.

R. Mesiar, E. Pap, and E. P. Klement. Triangular Norms. Springer, 2000.

P. Mongin. A Note on Mixture Sets in Decision Theory. Decision in Economics and Finance, pages $59-69,2000$.

B. Peleg. Utility Functions for Partially Ordered Topological Spaces. Econometrica, 38 (1):93-96, 1970.

J.-P. Penot and M. Volle. On Quasi-Convex Duality. Mathematics of Operations Research, 15:597-625, 1990a. 
J.-P. Penot and M. Volle. Inversion of Real-Valued Functions and Applications. Mathematical Methods of Operations Research, 34(2):117-141, march 1990b.

T. Rader. The Existence of a Utility Function to Represent Preferences. The Review of Economic Studies, 30(3):229-232, 1963.

L. J. Savage. The foundations of Statistics. Dover Publications, 2 revised edition, 1972.

W. Sharpe. Capital Asset Prices: A Theory of Market Equilibrium under Conditions of Risk. Journal of Finance, 19:425-442, 1964.

C. Skiadas. Conditioning and Aggregation of Preferences. Econometrica, 65(2):347-368, March 1997a.

C. Skiadas. Subjective Probability under Additive Aggregation of Conditional Preferences. Journal of Economic Theory, 76(2):242-271, Oct. 1997b.

J. von Neumann and O. Morgenstern. Theory of Games and Economics Behavior. Princeton University Press, 2nd edition, 1947.

S. Weber. Distribution-Invariant Risk Measures, Information, and Dynamic Consistency. Mathematical Finance, 16(2):419-441, April 2006. 



\section{Selbständigkeitserklärung}

Ich erkläre, dass ich die vorliegende Arbeit selbständig und nur unter Verwendung der angegebenen Literatur und Hilfsmittel angefertigt habe.

Berlin, den 28.01.2008

Samuel Drapeau 